\title{
The Ageing and Rejuvenation of Soft, Glassy \\ Complex Fluids
}

\author{
by \\ Simon Andrew Rogers
}

\author{
A thesis \\ submitted to the Victoria University of Wellington \\ in fulfilment of the \\ requirements for the degree of \\ Doctor of Philosophy \\ in Physics.
}

Victoria University of Wellington

2009 



\begin{abstract}
Suspensions of multiarm star polymers are studied as models for soft colloidal interactions in colloidal glasses. Establishing a pre-shearing protocol which ensures a reproducible initial state (the "rejuvenation" of the system), we report here the stress evolution from startup for two different concentrations for a range of shear rates using conventional rheological techniques. We show the existence of critical shear rates which are functions of the concentration. When the suspensions are sheared at rates below the critical rates, the stress rises to a common value which is also a function of the concentration. The system thus evolves into a yield stress-like fluid. This behavior manifests itself as an evolution from a monotonic, slightly shear-thinning flow curve to a flow curve dominated by a stress plateau.

Complementary to the controlled-rate measurements, stress-controlled measurements show that for a stress below the critical stress, the rate at which strain is acquired drops several orders of magnitude, providing evidence of a lower branch of the flow curve. In stress-controlled ageing experiments, the material recovers an increasing fraction of the strain acquired under stress with waiting time upon cessation of the (less than critical) stress. The freshly rejuvenated suspension recovers a mere $2 \%$ of the acquired strain, while for a waiting time of $10^{4} \mathrm{~s}$ the material recovers $97 \%$ of the acquired strain. The material thus appears to evolve from a nearly ideal fluid to a nearly ideal solid.

We relate this bulk evolution to spatially and temporally resolved Rheo-NMR velocity profiles which clearly show an evolution to a strongly shear-banded state. The velocity of the suspension in the lower shear band is below the uncertainty of the experiment. The growth of the (assumed) zero-shear band is well described by a Gompertz relation. The effects of shear-rate, temperature and waiting time on the Gompertz parameters are investigated.

Phenomenological understanding is provided through a scalar model that describes the stress-dependent free-energy landscape. Using a dual-minimum free-energy landscape, the model is able to replicate the behaviour of the stress after startup in shear-
\end{abstract}


rate controlled experiments, the flow curve and the velocity profiles across the gap of a Couette geometry.

The Large-Amplitude-Oscillatory-Shear (LAOS) response is reported along with discussions of current LAOS analysis techniques. The stress response to LAOS of the star suspensions is well described in a Cox-Merz manner by a modified Cross model. The modified Cross model highlights an asymmetry in the LAOS response. This constitutes the first ever report of asymmetric LAOS responses. The asymmetry is followed as a function of time using two complementary scalar variables. A speculative interpretation is given to account for the evolution of the asymmetry. 


\section{Acknowledgements}

This thesis would not exist without input from a number of people. Primarily, I acknowledge the assistance and supervision of Prof. Paul Callaghan. It has been Paul's guidance and sage advice, coupled with his unending energy that I feel I have benefitted from the most over the course of producing this thesis. While producing a thesis is part of the requirements of a PhD., I have always felt that the most important, unwritten, requirement of completing a PhD. is to learn how science works and how to be a successful scientist. In this vein, it has been an immense pleasure to have been supervised by such a successful scientist and science leader.

I also acknowledge the input of my co-supervisor A/Prof. Kathryn McGrath, whose in-depth and on-point questioning, I feel, has strengthened the work in this thesis.

Further acknowledgement is extended to Prof. Dimitris Vlassopoulos of the Foundation Of Research, Science and Technology, Hellas (FORTH), in Greece. Without Dimitris' help and suggestion to work on the star system as well as providing the stars, this thesis would have a dramatically different look. His friendship and infectious joy for science have made this collaboration not only rewarding and enjoyable, but a wonderful start to an academic career.

I'm indebted to Dr. Suzanne Fielding, whose passion and insight inspired me to pursue a PhD. in the field of soft matter. I have had numerous conversations with Suzanne regarding the work of this thesis, conversations that have always been of great scientific value.

Throughout the course of producing this thesis, I have had the pleasure of sharing lab space with a large number of gifted postdoctoral fellows and postgraduate students. The collaborative atmosphere in the lab meant that many problems and issues were discussed around a whiteboard or during one of our weekly group meetings. Those whose input I have benefitted from most are (in no particular order) Dr. Petrik Galvosas, Dr. Penny Hubbard, Dr. Kirk Feindel (postdoctoral fellows), Dr. Maria del 
Rosario Lopez Gonzalez, Dr. Antoine Lutti, Mark Hunter, Brad Douglass, Alan Raudsepp, Kate Washburn, Meghan Halse and Ben Parkinson (postgraduate students).

I've had a lot of 'behind-the-scenes' help from a number of people who have enabled me to carry out my science. Administrative help from Margaret Brown has been invaluable. Technical support and help from Rob Lenihan and Scott Forbes has meant my computers run smoothly and I have relevant software. Alan Rennie, Manu Pouajen-Blakiston and David Gilmour have been the 'go-to' guys when it came to building or modifying apparatus and Phillip Luey has maintained lab supplies, making sure, among other things, that our problem solving sessions always take place with working pens.

From a personal perspective, I would like to thank my parents, Kevin and Glenys, my brother Tim and his wife Debbie and my parents-in-law, Ruth and Henry. Thanks also goes to all of my friends and softball teammates. Explaining exactly what it is that I do to them has enabled me to work on my communication skills and often enhanced my own understanding. Answering their questions and passing on some of my own excitement has kept me grounded and always aware of the bigger picture.

The financial assistance of the Royal Society of New Zealand Marsden fund has ultimately made this all possible.

Lastly, I would like to acknowledge the love, support and editing assistance I have received from my wife Krista while producing this thesis. 


\section{Contents}

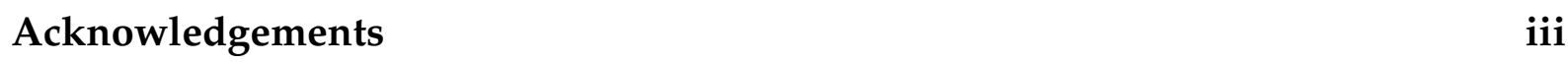

1 Introduction 1

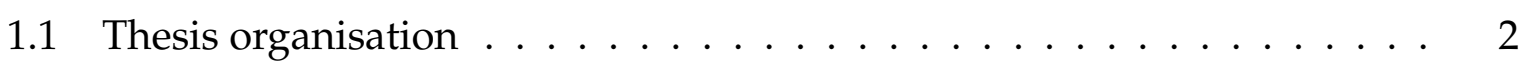

2 Rheology $\mathbf{5}$

2.1 Hookean Solids and Newtonian Liquids . . . . . . . . . . . . . . . . 6

2.1 .1 Hookean Solids . . . . . . . . . . . . . . . . 6

2.1.2 Newtonian Liquids . . . . . . . . . . . . . . . . . 7

2.2 Viscoelasticity ....................... 8

2.2.1 Maxwell Model . . . . . . . . . . . . . . . . . 9 9

2.2.2 Kelvin-Voigt Model . . . . . . . . . . . . . . . . . . . . . 10

2.3 Tensorial Representation of Stress and Strain . . . . . . . . . . . . . . 11

2.4 Linear Viscoelasticity . . . . . . . . . . . . . . . . . . . . . 14

2.4.1 Measurements of Linear Viscoelasticity . . . . . . . . . . . . . . 16

2.5 Non-Newtonian Fluids . . . . . . . . . . . . . . . . . . . . . . 20

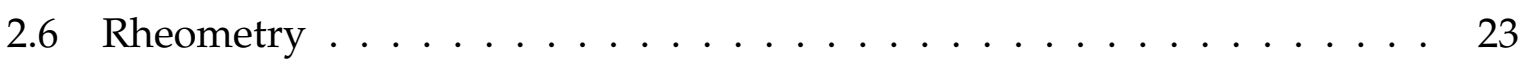

2.6 .1 Geometries ........................ 24

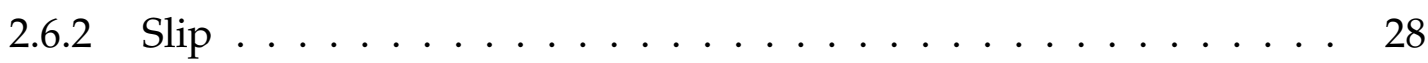

3 Nuclear Magnetic Resonance (NMR) 31

3.1 Nuclear spin and magnetic moment . . . . . . . . . . . . . . . . 31

3.2 Zeeman interaction . . . . . . . . . . . . . . . . 33

3.3 Other interactions . . . . . . . . . . . . . . . . 35

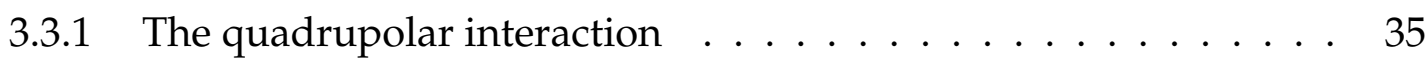

3.3.2 The dipolar interaction . . . . . . . . . . . . . 36 
3.3 .3 Chemical shift . . . . . . . . . . . . . . . 37

3.4 Bulk Magnetisation . . . . . . . . . . . . . . . . . . 38

3.5 Semi-classical description $\ldots \ldots \ldots$. . . . . . . . . . . . . 39

3.5.1 Excitation and the rotating frame . . . . . . . . . . . . . 40

3.6 Pulses . . . . . . . . . . . . . . . . . . . . . . 46

3.7 Relaxation . . . . . . . . . . . . . . . . . . 46

3.7.1 Spin-lattice relaxation . . . . . . . . . . . . . . . . 47

3.7.2 Spin-spin relaxation . . . . . . . . . . . . . . . . 49

3.7 .3 The Bloch equations . . . . . . . . . . . . . . . 50

3.8 Signal detection and the FID . . . . . . . . . . . . . . . . . . . 51

3.9 Signal averaging and phase cycling . . . . . . . . . . . . . . 52

3.10 Elementary pulse sequences . . . . . . . . . . . . . . . 53

3.10 .1 Spin and stimulated echoes . . . . . . . . . . . . . . . . . 54

3.10 .2 Measurements of nuclear relaxation . . . . . . . . . . . . . . 55

3.11 Imaging . . . . . . . . . . . . . . . . 57

3.11 .1 Spin density and k-space . . . . . . . . . . . . . . . . 57

3.11 .2 Selective pulses . . . . . . . . . . . . . . . . . . . 59

3.11 .3 Motion and q-space . . . . . . . . . . . . . . . . . . 59

3.11.4 Translational dynamics - self diffusion and flow . . . . . . . . . 61

4 Soft Matter $\quad 65$

4.1 Introduction . . . . . . . . . . . . . . . . 65

4.2 Experimental Systems . . . . . . . . . . . . . . . . 65

4.3 Behaviour of dense colloids . . . . . . . . . . . . . . . . 66

4.4 Ageing in soft matter . . . . . . . . . . . . . . . . . . 69

4.5 Heterogeneous flow in soft materials . . . . . . . . . . . . . . 70

4.6 Previous work on star polymer systems . . . . . . . . . . . . 70

5 Experimental: Conventional Rheology 73

5.1 Introduction . . . . . . . . . . . . . . . . . . 73

5.2 Determining the conditions for rejuvenation: Establishing a protocol . . 74

5.2.1 Nonlinear Strain Response to Oscillatory Shear . . . . . . . . . . 74

5.3 Transient response to continuous steady shear . . . . . . . . . . . . 77

5.3 .1 The Flow Curves . . . . . . . . . . . . . . . . . . . 84

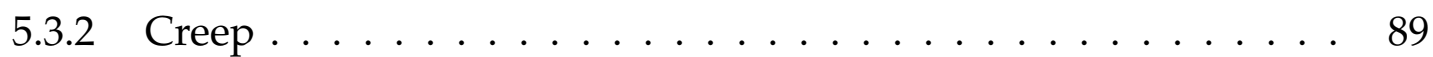


5.4 Stress Relaxation . . . . . . . . . . . . . . . . . . . . . . . . 91

5.5 Ageing under oscillatory flow . . . . . . . . . . . . . . 93

5.6 Conclusions . . . . . . . . . . . . . . . . . . 95

6 Experimental: Velocimetry 99

6.1 Introduction . . . . . . . . . . . . . . . . . . . . . . 99

6.2 Geometry . . . . . . . . . . . . . . . . . . . 99

6.3 Signal detection . . . . . . . . . . . . . . . . . . . . . 100

6.4 Experimental protocol . . . . . . . . . . . . . . . . . . . . 102

6.5 Evidence of shear banding . . . . . . . . . . . . . . . . . . . 104

6.6 Evolution of banded structure . . . . . . . . . . . . . . . . . . . . . . 104

6.7 Characterising the growth of the zero-shear band . . . . . . . . . . 107

6.8 Experimental parameter effects on banding evolution . . . . . . . . . . 108

6.8.1 Imposed shear rate effects . . . . . . . . . . . . . . . . . . . 108

6.8 .2 Waiting time effects . . . . . . . . . . . . . . . . . 111

6.8.3 Temperature effects . . . . . . . . . . . . . . . . . . . 115

6.9 Regarding the Gompertz equation . . . . . . . . . . . . . . . . . . 117

6.10 Structure of the 'zero-shear band' . . . . . . . . . . . . . . . . . . . . . . 118

6.11 Conclusions . . . . . . . . . . . . . . . . . . 120

$\begin{array}{lll}7 & \text { A theoretical approach } & 125\end{array}$

7.1 Introduction . . . . . . . . . . . . . . . . . . . . . 125

7.2 The trap model of Bouchaud . . . . . . . . . . . . . . . 126

7.3 The SGR theory . . . . . . . . . . . . . . . . . . . 127

7.4 A two-step model . . . . . . . . . . . . . . . . . . 130

7.4.1 Application of the model . . . . . . . . . . . . . . 132

7.5 Conclusions . . . . . . . . . . . . . . . . . . 137

8 Large-Amplitude Oscillatory Shear (LAOS) Response 139

8.1 Introduction . . . . . . . . . . . . . . . . . . . . . 139

8.2 Proposed method . . . . . . . . . . . . . . . . . . . 143

8.3 Parametrization . . . . . . . . . . . . . . . . . . 154

8.4 Symmetry of response . . . . . . . . . . . . . . . . . . . . . . 154

8.5 Total energy dissipation . . . . . . . . . . . . . . . 165

8.6 Discussion . . . . . . . . . . . . . . . . . . 171 
9 Conclusions $\quad 177$

9.1 Concluding remarks . . . . . . . . . . . . . . . . . 181

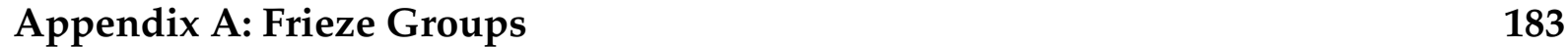

$\begin{array}{ll}\text { References } & 186\end{array}$ 


\section{Chapter 1}

\section{Introduction}

The dramatic complexity of the biological world, encompassing systems inherently far from equilibrium, is elucidated through the study of soft matter. Such studies have significance for many industrial applications and contain some science so bold and shocking that potential applications are still decades away.

"My own suspicion is that the universe is not only queerer than we suppose, but queerer than we can suppose." The British geneticist and evolutionary biologist J. B. S. Haldane's famous quote [1] alludes not only to science's main goal of replacing the queerness of the natural world with simple understanding, but it also makes clear his personal feelings that mankind may not be able to unravel the queer intricacies we see around us. While the latter point may forever remain an expression of personal opinion, the former is the driving force behind science.

The biological world that Haldane's work helped to shed light upon is rich in its queerness. It is a world where events occur on time scales that range from billionths of a second up to hours, where length scales range from billionths of a metre up to metres. It is a world where biology meets chemistry and physics in a scientific ménage á trois. The physical understanding of what happens in this amalgam of sciences, collectively referred to as 'soft matter science', has been the topic of much research and heated debate over the last two decades.

Soft matter is comprised primarily of polymers and polymer-like substances. Perhaps the most challenging and enlightening work has come from understanding the behaviour of these molecules that form a sort of 'shape spectrum' with long chains at one end and hard spheres at the other. Forming the intervening parts of this spectrum are branched polymers which are referred to as stars and pom-poms. While we can eas- 
ily imagine how these stars look (think of tiny pom-poms and koosh balls with varying numbers of arms), the queerness of the natural world rears its head when we examine how these materials respond to forces and strains. Most industrial applications and all biological occurrences take these materials far from their equilibrium configurations by applying strains and stresses.

The ultimate goal of this thesis is to elucidate the behaviour of such a system of star polymers when taken far from its equilibrium configuration via mechanical stresses and strains. While numerous techniques have been employed in the study of soft matter systems such as Dynamic Light Scattering (DLS), high frequency Ultrasonic Speckle Velocimetry (USV), Particle Imaging Velocimetry (PIV) and Laser Doppler Velocimetry (LDV) the results presented in this thesis have been gathered primarily from the use of traditional rheological methods. These methods often hint at, but cannot confirm, the existence of heterogenous flow in soft materials. In order to relate heterogeneous flow to the time dependent stress or strain rate constitutive properties, an experimental technique should combine rheomechanics with noninvasive velocimetry. In this thesis time-resolved nuclear magnetic resonance (NMR) microscopy is the velocimetry of choice. The use of nuclear magnetic resonance techniques is not limited solely to velocimetry; nuclear magnetic resonance spectroscopy adds molecular-level information to the macroscopic flow information.

\subsection{Thesis organisation}

It is necessary to divide such a body of work into discrete sections that present information that is gathered from a particular experimental technique, or relates to a particular behaviour. As such, this thesis has been divided into chapters with the aim of presenting the information in a logical progression. The first three chapters following this one are intended to give the reader an introduction to the experimental techniques used in the later chapters, to introduce the field of soft matter physics and in particular the star polymers studied in this work. The experimental and theoretical work that is the subject of this thesis is presented in the next four chapters with the conclusions of the thesis coming after these. In particular the layout of the thesis is as follows:

Chapter 2 introduces the concepts of rheology including providing the reader a definition of Hookean solids and Newtonian fluids. Chapter 2 also features an introduction to viscoelasticity and non-Newtonian fluids. The chapter is rounded out with 
a discussion of rheometry, the way rheological information is gathered, and factors a successful rheologist must be aware of.

Chapter 3 introduces the Zeeman interaction, magnetic resonance imaging and other concepts relating to nuclear magnetic resonance that will be necessary to understand the experimental results presented in later chapters.

Chapter 4 gives an introduction to the field of soft matter physics with a particular focus on ageing in soft matter. Star polymers are introduced, followed by a discussion on the need to study their flow and ageing behaviours.

Chapter 5 is the first chapter to contain the new work that is the focus of this thesis. The initial focus of this chapter is the determination of a protocol that is able to place the material in a well defined and reproducible starting state. The focus of this chapter then shifts to the rheological characterisation of the steady flow behaviour and how it changes as the material ages. In this chapter, a dramatic stiffening of the material is presented at shear rates and stresses below critical values. The results presented in this chapter are interpreted with respect to previously published results.

Chapter 6 focusses almost entirely on the evolution of the material in a concentriccylinder Couette geometry by the use of rheo-NMR velocimetry under different applied shear-rate, age and temperature conditions. These results are used in conjunction with spectroscopic information to add to the interpretation gained from the previous two chapters.

The thesis then takes a turn, and shifts the focus from experimental behaviours to understanding the results in terms of a coherent theoretical framework. Chapter 7 introduces a number of theoretical approaches that have proved to have varying degrees of success in terms of modelling the behaviour of soft materials and moves on to discuss a simple approach that mimics the dramatic experimental results presented previously.

In Chapter 8, the temporally changing response of the material to oscillatory flow is investigated with a particular emphasis on two novel analysis techniques for largeamplitude oscillatory shear responses. The changing shape of the response is interpreted with respect to the stiffening presented in the previous chapter.

Chapter 9 presents a summary of the conclusions from each chapter. 


\section{Chapter 2}

\section{Rheology}

When discussing how complex fluids behave, it is necessary to discuss their 'rheology'. What follows will be an introduction to the rheological concepts that are salient to this thesis and some points that are of interest to the author. The interested reader is directed to [2] for an excellent introduction to rheological tests and theory, [3] for a more thorough handling of fluid dynamics and [4] for a fairly complete discussion of the rheology of many types of complex fluids.

'Rheology' is the study of the flow and deformation of materials. The name comes from the Greek word oon meaning flow and 'ology' meaning study of. The name was coined by Professor Eugene Cook Bingham of Lafayette College, Indiana, who was the founding father of the Society of Rheology which was formed in 1929. The ideas of rheology are much older than that date suggests however and many cite the words of the Greek philosopher Heraclitus (born somewhere between 535 and 540 B.C. in Ephesos, and died 475 B.C.) as the first rheological expressions. Heraclitus is reported to have said

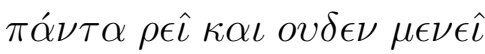

which translates as everything flows and nothing abides. Пর́ $\nu \tau \alpha \rho \epsilon \hat{\iota}$ is now the official motto of the Society of Rheology and features on every cover of the Society's own publication, the Journal of Rheology. This statement says that everything is a liquid, even though common sense tells us otherwise. The way out of this quandary had been given by the prophetess Deborah in the fifth chapter of the book of Judges in the Old Testament when, after defeating the Philistines she sang, "The mountains flowed 
before the Lord." Professor Marcus Reiner who worked with Bingham said [5]

"Deborah knew two things. First, that the mountains flow, as everything flows. But, secondly, that they flowed before the Lord, and not before man, for the simple reason that man in his short lifetime cannot see them flowing while the time of observation of God is infinite."

Thus he defined the dimensionless Deborah number as

$$
D_{e}=\tau / T
$$

where $\tau$ is a characteristic time of the material and $T$ is the time of observation. The difference between solids and liquids is then defined by the magnitude of $D_{e}$ : for large observation times or, conversely, very short characteristic times $\left(D_{e} \leq O\right.$ (unity)), the material will appear to flow whereas if the characteristic time is very long compared to the observation time $\left(D_{e} \gg 1\right)$, the material will act as a solid.

In the traditional sense, solids and liquids sit at opposite ends of a (discontinuous) spectrum. We learn at an early age that solids and liquids are different. As discussed in the next section, the works that describe these traditional views come from two men who achieved many advancements in their careers and whose views, like the types of materials they described, often sat at opposite ends of a spectrum.

\subsection{Hookean Solids and Newtonian Liquids}

\subsubsection{Hookean Solids}

In Robert Hooke's 1678 work “True Theory of Elasticity" he states

"the power of any spring is in the same proportion with the tension thereof"

This law is taught in high school science classes as 'Hooke's Law'. To modernise the language slightly, one may say in relation to springs if the force is doubled, the extension is doubled. Applying this idea to all solids we can say that if a force is applied to a solid block, the deformation achieved will be in direct proportion to that force. In three dimensions forces are applied to areas and so we define the stress, $\sigma$ applied to a material as $\sigma=F / A$, where $F$ is the force applied to an area $A$. Stress has the 


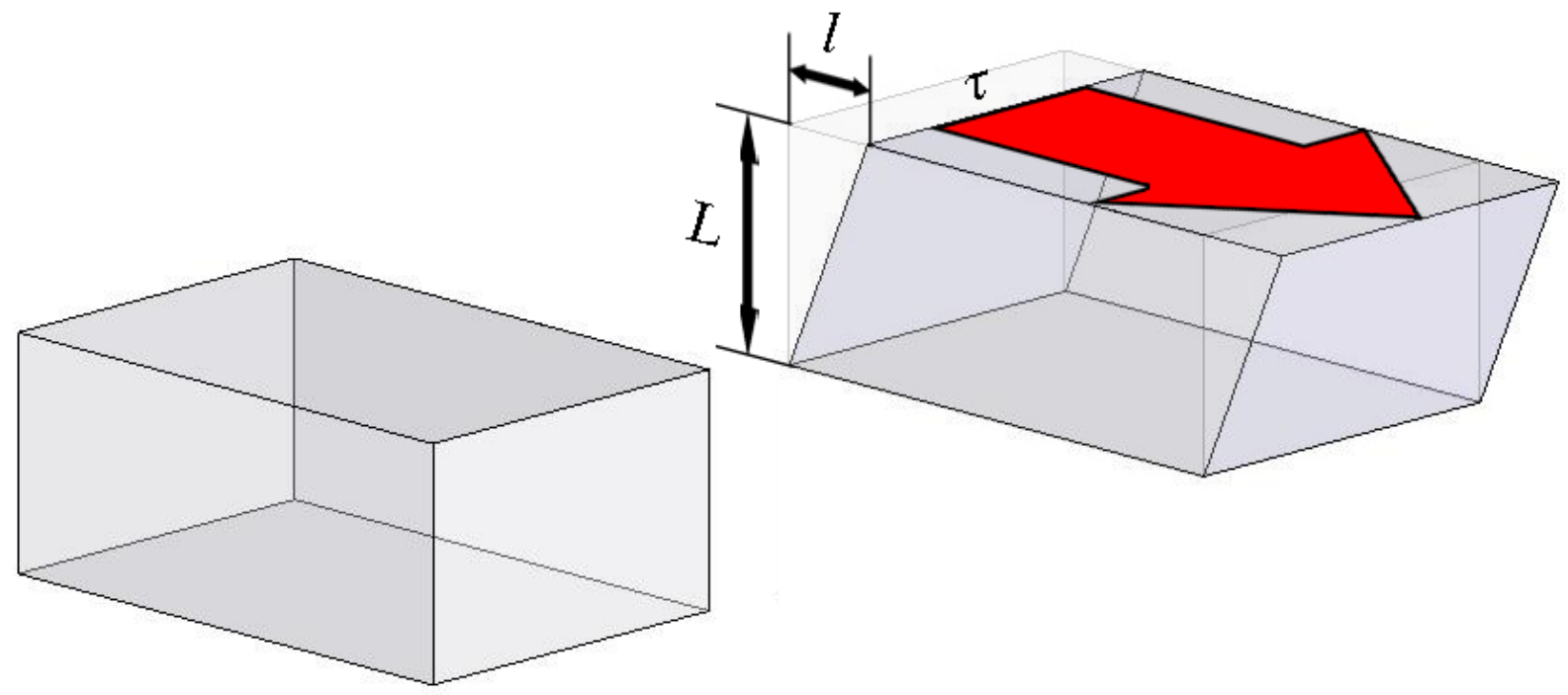

Figure 2.1: When a stress $\sigma$ is applied to a Hookean solid, an instantaneous deformation of magnitude $\gamma=l / L$ results. Upon cessation of the stress, the material returns to the original configuration. Here the stress is shown to act in one direction only.

familiar units of pressure, $P a$. Applying a stress of $\sigma$ to a Hookean solid yields an instantaneous deformation called the strain, denoted by $\gamma$. Strain is defined as the deformation relative to a reference configuration of length, area or volume [2]. Thus in Fig. 2.1 if the top of a block of height $L$ is moved by a linear amount $l$ relative to the bottom, a configuration known as shearing, the strain is equal to $l / L$. Strains are usually quoted in units of \% so that a strain of $l=L$ is referred to as $100 \%$. The amount of strain is related to the shear stress by the rigidity modulus or shear modulus of the material, $G$ (corresponding to the spring constant, $k$ in the familiar form of Hooke's Law $F=k x$ ) so that

$$
\sigma=G \gamma
$$

When a constant stress is applied to a Hookean solid, the spring-like solid deforms instantly by an amount $\gamma$ and remains deformed until the stress ceases in which case it immediately returns to its original conformation. It is common to refer to this type of behaviour as being elastic.

\subsubsection{Newtonian Liquids}

In his 1687 work "Philosophiae Naturalis Principia Mathematica" Isaac Newton wrote 


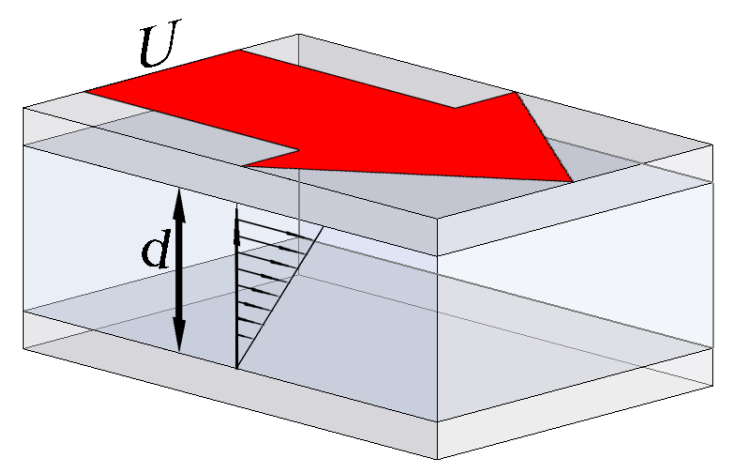

Figure 2.2: Two parallel plates in relative motion create a velocity gradient in the fluid between them equal to $U / d$.

"The resistance which arises from the lack of slipperyness of the parts of the liquid, other things being equal, is proportional to the velocity with which the parts of the liquid are separated from one another."

The velocity with which the parts of the liquid are separated from one another is known as the shear rate, $\dot{\gamma}$ (where the usual notation of $\dot{a}=\partial a / \partial t$ applies). In the simple case of two parallel plates with relative velocity $U$ as in Fig. 2.2 the shear rate is equal to the velocity gradient $U / d$. The lack of slipperyness is now called the 'viscosity' and is often denoted by $\eta$. Viscosity is defined by the relation

$$
\sigma=\eta \dot{\gamma}
$$

When a constant stress is applied to a Newtonian liquid, it deforms instantly and continuously at a rate $\dot{\gamma}$ until the applied stress ceases. The strain attained over the time in which the stress was applied is not recovered. It is common to refer to this type of behaviour as being viscous.

\subsection{Viscoelasticity}

In the previous section we introduced the ideas of viscous and elastic behaviours. Viscous materials were defined to be materials that respond to constant stresses, $\sigma$, by flowing at a constant rate $\dot{\gamma}$ until the stresses are ceased. Elastic materials were defined 


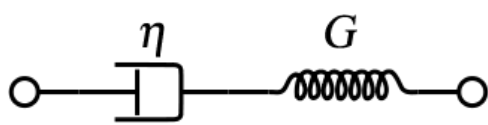

Figure 2.3: A schematic representation of the Maxwell model. A dashpot with viscosity $\eta$ is connected in series with a spring with elastic modulus $G$.

to be materials that instantly acquire strain when stressed and instantly recover that strain when the stresses are ceased. These are both ideal behaviours and it can be argued that all real materials exhibit a mixture of the two as suggested by equation 2.1. This mixture of viscous and elastic behaviours is referred to as viscoelasticity.

\subsubsection{Maxwell Model}

A simple model of a viscoelastic material was proposed by James Clerk Maxwell in a paper entitled "On the dynamical theory of gases" in 1867. The model consists of a viscous element called a dashpot in series with an elastic element, a spring and is shown in Fig. 2.3. The dashpot is described by a viscosity, $\eta$, and the spring by an elastic modulus, $G$. The Maxwell model has the property that strains, and hence strain-rates are additive. This property allows us to simply sum the strain-rates of the two elements to obtain the total strain-rate of the model [2],

$$
\dot{\gamma}_{\text {total }}=\dot{\gamma}_{V}+\dot{\gamma}_{E}
$$

We can rewrite this using equations 2.2 and 2.3 as

$$
\dot{\gamma}_{\text {total }}=\frac{\sigma}{\eta}+\frac{\dot{\sigma}}{G}
$$

The stress response to a strain, or strain-rate of the Maxwell model can be found by introducing the time constant $\tau_{M}=\frac{\eta}{G}$ and rearranging equation 2.5 to yield

$$
\sigma+\tau_{M} \dot{\sigma}=\eta \dot{\gamma}
$$

In the case of the application of a constant strain-rate, $\dot{\gamma}_{0}$, at time $t=0$ in which the strain rate is applied for all time $t>0$, we can solve equation 2.6 to show

$$
\sigma(t)=\eta \dot{\gamma}_{0}\left[1-\exp \left(\frac{-t}{\tau_{M}}\right)\right] .
$$




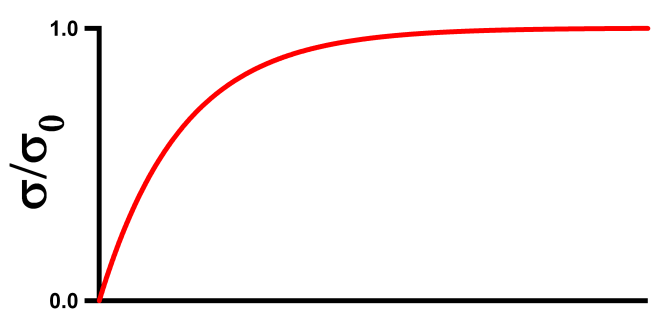

(a)

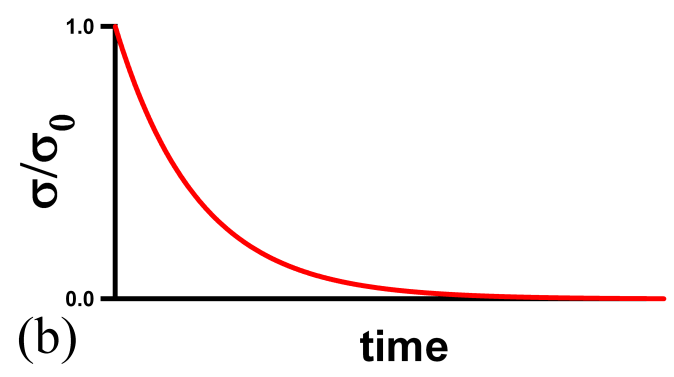

(b)

\section{time}

Figure 2.4: Stress evolution upon startup (a) and cessation (b) of shear in a Maxwell model where $\sigma_{0}=\eta \dot{\gamma_{0}}$.

This result means that the stress does not immediately rise to a steady value, rather it approaches a steady value with a time constant $\tau_{M}$ as shown in Fig. 2.4(a). It can also be shown that if a shear rate, $\dot{\gamma}_{0}$, has existed for times $t<0$ and ceases at $t=0$ the stress relaxes as

$$
\sigma(t)=\eta \dot{\gamma}_{0} \exp \left(\frac{-t}{\tau_{M}}\right)
$$

for times $t \geq 0$. Thus the stress relaxes exponentially from the equilibrium value of $\eta \dot{\gamma_{0}}$ to 0 with relaxation time $\tau_{M}$ as shown in Fig. 2.4(b).

If a constant stress, $\sigma_{0}$ is applied to the Maxwell model instead of a constant strainrate at time $t=0$, the strain acquired can be expressed as

$$
\gamma(t)=\frac{\sigma_{0}}{G}+\frac{\sigma_{0}}{\eta} t
$$

where the first term describes the strain acquired by (the extension of) the elastic element and the second term describes the strain acquired by the viscous element. When the stress is ceased, the strain acquired by the elastic element, $\sigma_{0} / G$, is recovered immediately whereas the strain acquired by the viscous element, $\left(\sigma_{0} / \eta\right) t$ is not. Because the long time characteristics of the Maxwell model approach those of Newtonian liquids, it is said that the Maxwell model describes a viscoelastic liquid.

\subsubsection{Kelvin-Voigt Model}

Another simple viscoelastic model is named after the British physicist and engineer William Thomson, 1st Baron Kelvin and German physicist Woldemar Voigt and can be represented as a spring of elastic modulus $G$ in parallel with a dashpot of viscosity $\eta$. A schematic representation of this model is shown in Fig. 2.5. 


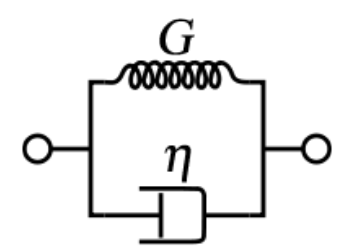

Figure 2.5: A schematic representation of the Kelvin-Voigt model. A dashpot with viscosity $\eta$ is connected in parallel with a spring with elastic modulus $G$.

In this model the stresses are additive [2] so that

$$
\sigma_{\text {total }}=\sigma_{V}+\sigma_{E}
$$

which can be rewritten as

$$
\sigma_{\text {total }}=G \gamma+\eta \dot{\gamma}
$$

It is easy to imagine that when a stress $\sigma_{0}$ is suddenly applied to the Kelvin-Voigt model that the spring will eventually reach the strain $\sigma_{0} / G$ but the evolution of the strain will be retarded by the dashpot. That is, equation 2.11 can be solved to show

$$
\gamma(t)=\frac{\sigma}{G}\left[1-\exp \left(\frac{-t}{\tau_{K V}}\right)\right]
$$

where $\tau_{K V}=\eta / G$ as before. We can likewise solve for the case where after a time $t^{*}>0$ the stress is ceased to find that the stress in the Kelvin model relaxes as

$$
\gamma(t)=\gamma\left(t^{*}\right) \exp \left(\frac{-t}{\tau_{K V}}\right)
$$

which confirms that in both sudden startup and sudden cessation of an applied external stress, the strain acquired or recovered by the Kelvin model is retarded in time by the time constant $\tau_{K V}$ as shown in Fig. 2.6. Because the long time characteristics of the Kelvin-Voigt model approach those of Hookean solids, it is said that the Kelvin-Voigt model describes a viscoelastic solid.

\subsection{Tensorial Representation of Stress and Strain}

We have thus far dealt with stresses and strains as scalar quantities used to describe one-dimensional dynamics. In reality, stresses and strains are applied to volumes of 


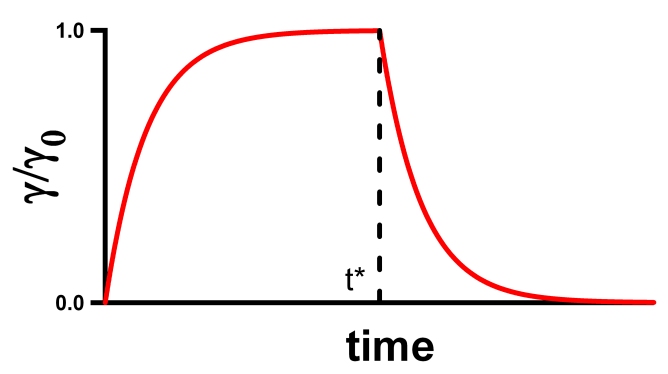

Figure 2.6: Strain evolution upon startup and cessation of stress in the Kelvin-Voigt model where $\gamma_{0}=\sigma_{0} / G$.

material which respond in three spatial dimensions. In the following discussion of the tensorial description of stresses and strain the nomenclature used in Larson [4] shall be adhered to.

We shall begin by defining the vector $\mathbf{x}=\left(x_{1}, x_{2}, x_{3}\right)$ to be a point in three-dimensional space with Cartesian coordinates $x_{1}, x_{2}$ and $x_{3}$. The velocity vector at a point $\mathbf{x}$ is defined as being $\mathbf{v}=\left(v_{1}(\mathbf{x}), v_{2}(\mathbf{x}), v_{3}(\mathbf{x})\right)$ where $v_{1}$ is the component of the velocity parallel to direction " 1 " and likewise for $v_{2}$ and $v_{3}$. The velocity gradient tensor $\nabla \mathbf{v}$, which describes the steepness of velocity variation is then given by the array [4]

$$
\nabla \mathbf{v}=\left(\begin{array}{lll}
\frac{\partial v_{1}}{\partial x_{1}} & \frac{\partial v_{2}}{\partial x_{1}} & \frac{\partial v_{3}}{\partial x_{1}} \\
\frac{\partial v_{1}}{\partial x_{2}} & \frac{\partial v_{2}}{\partial x_{2}} & \frac{\partial v_{3}}{\partial x_{2}} \\
\frac{\partial v_{1}}{\partial x_{3}} & \frac{\partial v_{2}}{\partial x_{3}} & \frac{\partial v_{3}}{\partial x_{3}}
\end{array}\right)
$$

The velocity gradient tensor of simple planar Couette flow depicted in Fig. 2.2 thus only has one non-zero component, $v_{1}$, and it varies in one direction only, the direction orthogonal to the plates. In this case, the velocity gradient tensor is [4]

$$
\nabla \mathbf{v}=\left(\begin{array}{ccc}
0 & 0 & 0 \\
\dot{\gamma} & 0 & 0 \\
0 & 0 & 0
\end{array}\right)
$$

where $\dot{\gamma} \equiv \partial v_{1} / \partial x_{2}$.

The symmetric part of the velocity gradient is called the rate-of-deformation tensor and is denoted $\mathbf{D}[4]$ :

$$
2 \mathbf{D} \equiv \nabla \mathbf{v}+(\nabla \mathbf{v})^{T}
$$




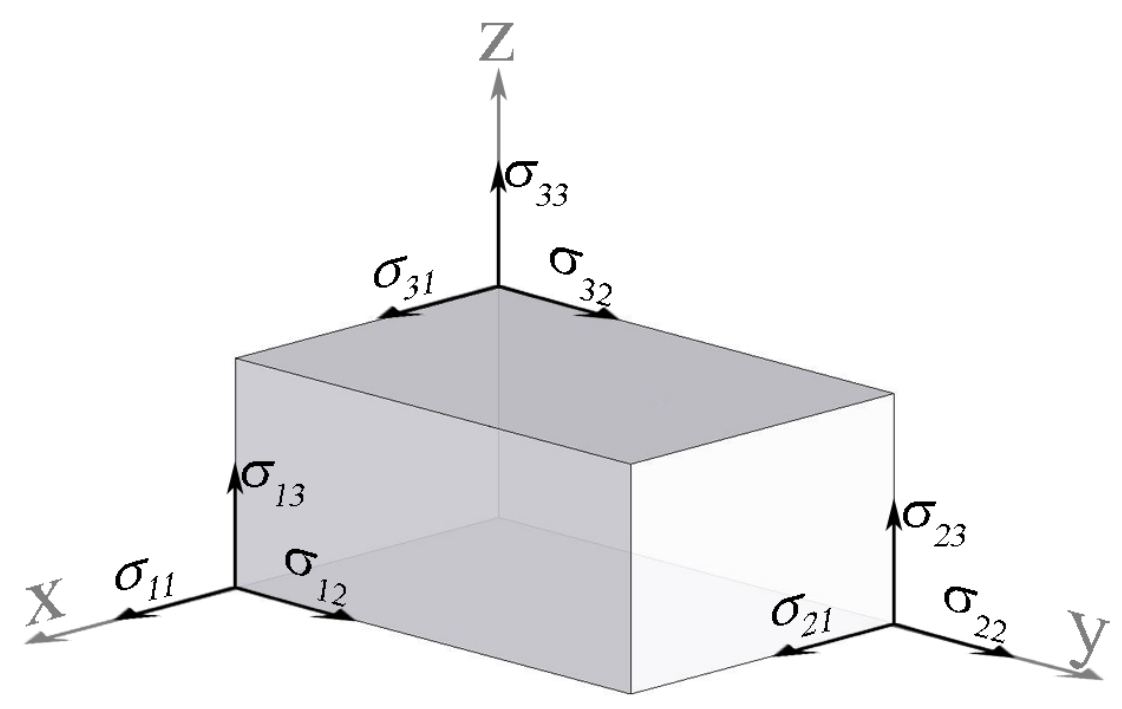

Figure 2.7: Components of the extra stress tensor visualized on a finite volume element. The first subscript refers to the normal of the face and the second subscript refers to the direction along which the stress acts.

We express the three-dimensional stress as the state-of-stress tensor:

$$
\mathbf{T}=\left(\begin{array}{lll}
F_{11} & F_{12} & F_{13} \\
F_{21} & F_{22} & F_{23} \\
F_{31} & F_{32} & F_{33}
\end{array}\right)
$$

where $F_{i j}$ is the component of the total force acting on a face perpendicular to the $i$ th direction in the $j$ th direction. $\mathbf{T}=\sigma-p \mathbf{1}$ is a symmetric tensor in all but the most unusual cases [4]. $\sigma$ is the extra stress tensor, $p$ is the hydrostatic pressure and $\mathbf{1}$ is the identity tensor of the same rank. Using this convention, it should be noted that a positive hydrostatic pressure results in a negative stress. Stresses that exist in addition to a hydrostatic pressure are expressed as the extra stress tensor, $\sigma$. The components of the extra stress tensor are denoted $\sigma_{i j}$ and represent a component of stress acting in the $j^{\text {th }}$ direction on the elemental plane whose normal is in the $i^{\text {th }}$ direction. This is visualized in Fig. 2.7. It is common to refer to the components $\sigma_{i i}$ as the normal components or normal stresses because they act in the same direction as the normals to the faces (these are often just written as $\sigma_{i}$, a convention that will not be adhered to in this work) and the components $\sigma_{i j},(i \neq j)$ as the shear components or shear stresses because they act perpendicular to the normals. In the case of an incompressible fluid, 
it is possible to completely define the stress by the three shear stresses $\sigma_{12}, \sigma_{13}, \sigma_{23}$ and the normal stress differences where the first normal stress difference is defined as $N_{1} \equiv$ $\sigma_{11}-\sigma_{22}$ and the second as $N_{2} \equiv \sigma_{22}-\sigma_{33}$. If the volume is finite then, strictly, these are average stresses. In the limit when the volume approaches zero, the stresses become stresses at the point that lies at the centre of the volume. In general, stress varies from point to point and so is a tensor field.

The stress in a complex fluid depends not only on the instantaneous velocity gradient, but also on the time over which that velocity gradient has been imposed. Consider the point in the fluid that at a past time $t^{\prime}$ occupied position $\mathbf{x}^{\prime}$ but at a time $t$ resides at the point $\mathbf{x}$. Using similar notational conventions to represent orientations, we express the deformation history of the material using the tensor $\mathbf{E}$ :

$$
\mathbf{E}\left(t^{\prime}, t\right) \equiv \frac{\partial \mathbf{x}}{\partial \mathbf{x}^{\prime}}=\left(\begin{array}{lll}
\frac{\partial x_{1}}{\partial x_{1}^{\prime}} & \frac{\partial x_{2}}{\partial x_{1}^{\prime}} & \frac{\partial x_{3}}{\partial x_{1}^{\prime}} \\
\frac{\partial x_{1}}{\partial x_{2}^{\prime}} & \frac{\partial x_{2}}{\partial x_{2}^{\prime}} & \frac{\partial x_{3}}{\partial x_{2}^{\prime}} \\
\frac{\partial x_{1}}{\partial x_{3}^{\prime}} & \frac{\partial x_{2}}{\partial x_{3}^{\prime}} & \frac{\partial x_{3}}{\partial x_{3}^{\prime}}
\end{array}\right)
$$

The tensor $\mathbf{E}$ contains information on the rotation of the fluid in addition to the stretching of the fluid element in each of its three dimensions. If a material is rotated only and is not stretched, there are no extra stresses induced. In order to remove the solid-body rotational information from $\mathbf{E}$ to leave us with only the stress-causing stretching, we define the Finger tensor, $\mathbf{B}$ :

$$
\mathbf{B} \equiv \mathbf{E}^{T} \cdot \mathbf{E}
$$

For the case of simple shear illustrated in Fig. 2.1, the Finger tensor is [4]

$$
\mathbf{B}=\left(\begin{array}{ccc}
1+\gamma^{2} & \gamma & 0 \\
\gamma & 1 & 0 \\
0 & 0 & 1
\end{array}\right)
$$

where $\gamma=\gamma\left(t^{\prime}, t\right)$ is the strain accumulated between times $t^{\prime}$ and $t$.

The results of sections 2.1 and 2.2 can thus be generalized to the respective tensorial quantities.

\subsection{Linear Viscoelasticity}

In section 2.2 viscoelasticity was mentioned as a combination of viscous and elastic properties. In this section we formalize the idea of viscoelasticity and introduce the 
concepts of linear and non-linear viscoelasticity.

The behaviour of viscoelastic materials under certain conditions, which will be defined shortly, can be described by linear differential equations in time with constant coefficients. If the coefficients change with a parameter other than time, the behaviour is no longer linear, it is said to be non-linear. We can express a general relation between stress and strain in linear viscoelastic materials as

$$
\left(1+\alpha_{1} \frac{\partial}{\partial t}+\alpha_{2} \frac{\partial^{2}}{\partial t^{2}}+\ldots+\alpha_{n} \frac{\partial^{n}}{\partial t^{n}}\right) \sigma=\left(\beta_{0}+\beta_{1} \frac{\partial}{\partial t}+\beta_{2} \frac{\partial^{2}}{\partial t^{2}}+\ldots+\beta_{m} \frac{\partial^{m}}{\partial t^{m}}\right) \gamma
$$

where $n=m$ or $n=m-1$. We have reverted back to the scalar representation of stress and strain for simplicity and we have assumed the simple type of shear as shown in Fig. 2.2. It is possible to use the same generalized linear viscoelastic equation for other types of shear by replacing the scalar quantities with their tensorial counterparts. Note that equation 2.20 is written in terms of ordinary partial derivatives which further restricts the bounds of the linear theory to small changes in the variables.

If we assume the only non-zero coefficient in equation 2.20 is $\beta_{0}$, we end up with

$$
\sigma=\beta_{0} \gamma
$$

which is the same as equation 2.2 for a Hookean solid, where $\beta_{0}=G$, the rigidity modulus. If $\beta_{1}$ is the only non-zero term in equation 2.20 , we obtain

$$
\sigma=\beta_{1} \frac{\partial \gamma}{\partial t}
$$

which is the same as equation 2.3, which describes a Newtonian liquid of viscosity $\eta=\beta_{1}$. If we now allow $\beta_{0}$ and $\beta_{1}$ to be the only non-zero coefficients and write them in the more familiar ways just described, equation 2.20 becomes

$$
\sigma=G \gamma+\eta \dot{\gamma}
$$

which is the Kelvin-Voigt model we derived in equation 2.11 through purely geometric arguments. It comes as no surprise then, that the Maxwell model of equation 2.5 can also be obtained from equation 2.20 by setting $\alpha_{1}=\tau_{M}$ and $\beta_{1}=\eta$ as the only non-zero coefficients.

The generalized Maxwell model assumes a number of relaxation processes take place in real materials and accounts for this by a parallel connection of a finite number 
$N$, or an enumerable infinity of Maxwell elements. Assuming a continuous distribution of relaxation times leads to an expression of the stress as a function of time as a homogeneous Volterra equation of the first type:

$$
\sigma(t)=\int_{-\infty}^{t} G\left(t-t^{\prime}\right) \dot{\gamma}\left(t^{\prime}\right) d t^{\prime}
$$

where $G\left(t-t^{\prime}\right)$ is called the relaxation modulus. In the case of dilute complex fluids where one can think of the Maxwell elements as being dilute in the solvent, there will be a need to express the stress as a function of time in terms of an inhomogeneous Volterra equation of the first type:

$$
\sigma(t)=\eta_{s} \dot{\gamma}+\int_{-\infty}^{t} G^{(M)}\left(t-t^{\prime}\right) \dot{\gamma}\left(t^{\prime}\right) d t^{\prime}
$$

where the first term represents the property of the solvent and the second term represents the contribution from the Maxwell elements. If we measure the shear strain from $t=0$, we can integrate equation 2.24 by parts:

$$
\begin{aligned}
\sigma(t) & =\int_{-\infty}^{t} G\left(t-t^{\prime}\right) \dot{\gamma}\left(t^{\prime}\right) d t^{\prime} \\
& =\left[G\left(t-t^{\prime}\right) \gamma\left(t^{\prime}\right)\right]_{-\infty}^{t}-\int_{-\infty}^{t} \frac{\partial G\left(t-t^{\prime}\right)}{\partial t^{\prime}} \gamma\left(t^{\prime}\right) d t^{\prime} \\
& =-\int_{-\infty}^{t} M\left(t-t^{\prime}\right) \gamma\left(t, t^{\prime}\right) d t^{\prime}
\end{aligned}
$$

where $M\left(t-t^{\prime}\right)=\frac{\partial}{\partial t^{\prime}} G\left(t-t^{\prime}\right)$ is called the memory function. An important feature of both equations 2.24 and 2.26 is that they equate functions that relate to the material properties viscosity and relaxation time as well as the flow properties of shear rate, and equivalently deformation.

\subsubsection{Measurements of Linear Viscoelasticity}

Measurements of linear viscoelastic properties are divided into two distinct methods which are classed as either static or dynamic. Static measurements consist of step-wise applications of constant stresses/strains while the strain/stress response of the material is recorded as a function of time. Dynamic measurements involve an oscillatory application of strain where the stress response is recorded. 

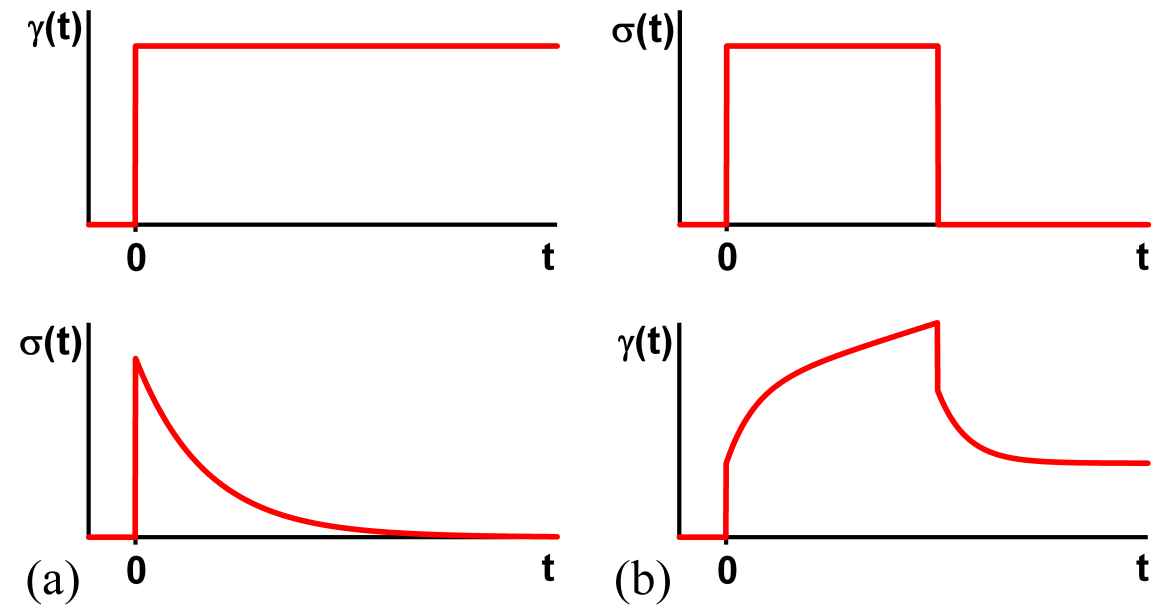

Figure 2.8: Static methods of measuring viscoelastic properties. (a) A step-wise application of strain top results in a stress relaxation bottom. (b) A step-wise application of stress top results in an initial instantaneous acquisition of strain, followed by a period of retarded growth and then a steady increase until the stress ceases. Upon cessation, an identical instantaneous amount of strain is recovered. The strain acquired in the steady period is not recovered.

Consider the case where a constant (small) strain $\gamma$ is applied step-wise to a material at a time $t^{\prime}=0$ as shown in Fig. 2.8(a). We can replace $\dot{\gamma}\left(t^{\prime}\right)$ in equation 2.24 with a Dirac delta function $\gamma_{0} \delta\left(t^{\prime}\right)$. Equation 2.24 then reduces to

$$
\sigma(t)=\gamma_{0} G(t)
$$

which provides a direct determination of $G(t)$. In the case where $G(t)$ is constant, this is identically the behaviour of a Hookean solid. If, instead of applying a constant strain, a constant strain rate, $\dot{\gamma}$ is applied in a step-like manner, then equation 2.24 becomes

$$
\sigma(t)=\dot{\gamma} \int_{-\infty}^{t} G\left(t-t^{\prime}\right) d t^{\prime}=\dot{\gamma} \int_{0}^{\infty} G(t) d t
$$

From equation 2.28 we can thus relate $\eta(t)=\int_{0}^{t} G(t)$, and in the case where this is a constant it is said that the material behaves as a Newtonian fluid.

A test that involves an application of a constant stress is referred to as a creep experiment. A typical creep experiment is depicted in Fig. 2.8(b). A constant stress is applied to the material in a step-wise manner (Fig. 2.8(b) top) and the strain is recorded as a function of time (Fig. 2.8(b) bottom). There is an initial instantaneous (as near to instantaneous as is possible due to the experimental geometry having some finite inertia) 
acquisition of strain which is the elastic response of the material. There is then a period where the acquisition of strain is retarded which is, in turn, followed by a period of time where the material flows (acquires strain) at a constant rate. Upon cessation of the stress at some later time, the elastic strain is recovered. The strain acquired during steady flow is not.

As previously mentioned, the dynamic method of measuring linear viscoelastic properties consists of an oscillatory application of strain while simultaneously recording the stress response of the material. The strain and strain rate are thus

$$
\gamma(t)=\gamma_{0} \sin (\omega t), \dot{\gamma}(t)=\gamma_{0} \omega \cos (\omega t)=\gamma_{0} \omega \Re[\exp (i \omega t)]
$$

where $\gamma_{0}$ is the amplitude of oscillation and $\omega$ is the (angular) frequency and $\Re$ refers to the real part. Substituting these terms into equation 2.24 yields

$$
\begin{aligned}
\sigma(t) & =\int_{-\infty}^{t} \gamma_{0} \omega \cos \left(\omega t^{\prime}\right) G\left(t-t^{\prime}\right) d t^{\prime} \\
& =\gamma_{0} \omega \int_{0}^{\infty} \cos \left(\omega\left[t-t^{\prime \prime}\right]\right) G\left(t^{\prime \prime}\right) d t^{\prime \prime} \\
& =\gamma_{0}\left[\omega \int_{0}^{\infty} \sin \left(\omega t^{\prime \prime}\right) d t^{\prime \prime} \sin (\omega t)+\omega \int_{0}^{\infty} \cos \left(\omega t^{\prime \prime}\right) G\left(t^{\prime \prime}\right) d t^{\prime \prime} \cos (\omega t)\right] \\
& =\gamma_{0}\left[G^{\prime} \sin (\omega t)+G^{\prime \prime} \cos (\omega t)\right]
\end{aligned}
$$

where $G^{\prime}$ and $G^{\prime \prime}$ are called the storage modulus and loss modulus, respectively and represent the in- and out-of-phase response of the material. It can be seen from equations 2.2 and 2.3 that an in-phase response corresponds to solid-like behaviour and and outof-phase response corresponds to liquid-like behaviour. In general terms then, the storage modulus represents the solid-like response of a material and the loss modulus represents the liquid-like response. The storage and loss moduli can be combined to give the complex modulus, $G^{*}(\omega)$ :

$$
G^{*}(\omega)=G^{\prime}(\omega)+i G^{\prime \prime}(\omega)
$$

This behaviour can be expressed in another way where it is assumed that the stress response to an oscillatory strain can be expressed in the same form with a phase in advance of the strain by an amount $\delta$, called the loss angle. Recalling equation 2.29 the stress is then

$$
\sigma(t)=\sigma_{0} \Re[\exp (i(\omega t+\delta))]
$$



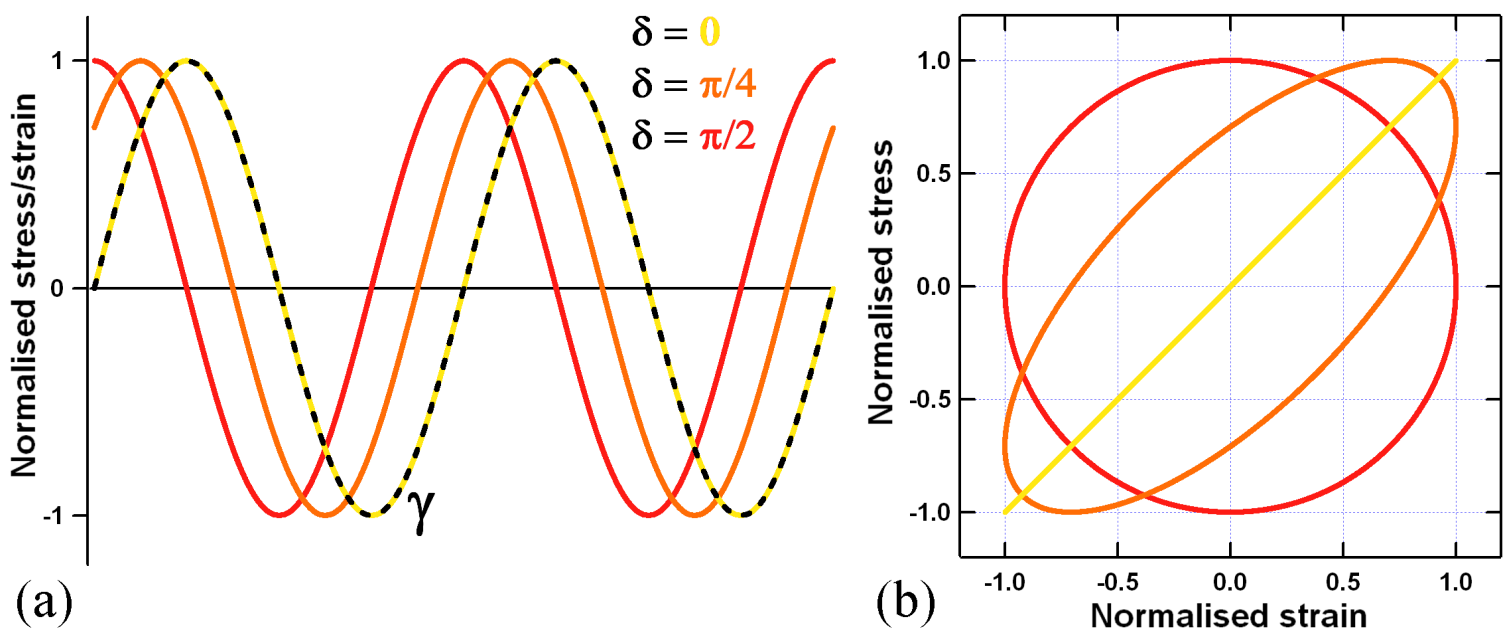

(a)

(b)

Figure 2.9: Dynamic method of measuring viscoelastic properties. (a) Stress response (solid lines) calculated from equation 2.32 compared with the strain input (dashed line) as functions of time for phase differences (light to dark) $\delta=0, \pi / 4, \pi / 2$. (b) Parametric Lissajous curves displaying the normalized stress as a function of the normalized strain for the same phase shifts.

where

$$
\tan \delta=G^{\prime \prime} / G^{\prime}
$$

This relation is displayed in the left of Fig. 2.9, where the normalized-strain input as a function of time is depicted as a dashed line while the normalized-stress output as a function of time is displayed as a solid red line for loss angles of $\delta=0^{\circ}$ (corresponding to a purely elastic response), $45^{\circ}$ (a viscoelastic response that is an equal mixture of elastic and viscous responses) and $90^{\circ}$ (corresponding to a purely viscous response). The right of Fig. 2.9 displays the same information in a series of Lissajous curves, named after the French mathematician Jules Antoine Lissajous (March 4, 1822 - June 24,1880). A general Lissajous curve is the graph of the system of parametric equations $x=A \sin (a t+\delta), y=B \sin (b t)$. In rheological Lissajous figures the abscissa (x-coordinate) is the (normalized) strain and the ordinate (y-coordinate) is the (normalized) stress. Lissajous curves have the immediately recognizable benefit, compared to plots of the input and output variables as functions of time, of compactness. A Lissajous curve of purely Hookean elastic behaviour is a straight line whose gradient is the elastic modulus and the Lissajous curve of purely Newtonian viscous behaviour is an ellipse where the ratio of semimajor axis to semiminor axis is $\eta \omega$. 
The area enclosed by a rheological Lissajous curve is equal to the energy dissipated per unit volume per cycle, $E_{d}$. That is

$$
E_{d}=\oint \sigma d \gamma=\oint \sigma(t) \dot{\gamma}(t) d t
$$

which, using equation 2.30 , can be expressed as

$$
\begin{aligned}
E_{d} & =\gamma_{0}^{2} \omega \int_{0}^{2 \pi / \omega}\left[G^{\prime} \sin (\omega t)+G^{\prime \prime} \cos (\omega t)\right] \cos (\omega t) d t \\
& =\gamma_{0}^{2} \omega G^{\prime \prime} \int_{0}^{2 \pi / \omega} \cos ^{2}(\omega t) d t \\
& =\gamma_{0}^{2} \pi G^{\prime \prime}
\end{aligned}
$$

This result shows that the dissipative nature of a material depends on only one material function, $G^{\prime \prime}$.

In summary, the dynamic response of a material to a sinusoidally oscillating strain is said to be linear providing the dynamic moduli, the storage and loss moduli, show no amplitude dependence and the stress response is sinusoidal. Typically, nonlinearities appear above a critical strain amplitude $\gamma_{0}$. Nonlinear oscillatory flow is often referred to as Large-Amplitude-Oscillatory-Shear (LAOS) because of this.

\subsection{Non-Newtonian Fluids}

The discussion of fluid behaviour up to this point has been concerned with fluids that obey a linear relation between shear rate and shear stress so that

$$
\sigma=\eta \dot{\gamma}
$$

The coefficient of proportionality is called the viscosity and is a measure of the 'slipperiness' of a fluid. Equation 2.36 implies a constant viscosity for all applied shear rates/shear stresses. For many fluids, this behaviour is exhibited only over a range of shear rates/shear stresses, and for some fluids, it is not exhibited at all. In general, $\eta=\eta(\dot{\gamma})$. Fluids which show behaviours that deviate from equation 2.36 are referred to as non-Newtonian fluids. The next section will introduce some simple models of nonNewtonian fluids, starting with a model first introduced by Malcolm Cross in 1965 [6] from which a number of important models can be simply derived. The Cross model 
assumes that a type of irreversible flow is associated with the formation and rupture of structural linkages in non-Newtonian fluids and describes a viscosity that varies as

$$
\eta(\dot{\gamma})=\eta_{\infty}+\frac{\eta_{0}-\eta_{\infty}}{1+(C \dot{\gamma})^{m}}
$$

where $\eta_{0}$ and $\eta_{\infty}$ are the asymptotic values of the viscosity at very low and very high shear rates. $C$ is known as the Cross time constant and has units of time. The inverse of $C$ is a shear rate that indicates the change from $\eta_{0}$ towards $\eta_{\infty} . m$ is a dimensionless number that is called the (Cross) rate constant and is a measure of the degree of dependence of viscosity on shear rate in the region between $\eta_{0}$ and $\eta_{\infty}$. When $m=0$, the Cross model reduces to that of Newton.

If $\eta \ll \eta_{0}$ and $\eta \gg \eta_{\infty}$ such that we can assume $\eta_{0}-\eta \approx \eta_{0}$ and $\eta-\eta_{\infty} \approx \eta$, then the Cross model reduces to

$$
\eta(\dot{\gamma})=K \dot{\gamma}^{n-1}
$$

called the power law model. In the power law model the coefficient $K$ is called the consistency index, with units of $P a . s^{n}$ and $n$ the (dimensionless) power-law exponent. When the power-law index $n$ is identically 1 , Newtonian behaviour is recovered with the consistency index $K=\eta$. If $n<1$, the viscosity lowers as higher shear rates are applied and the material is said to be shear thinning. Likewise when $n>1$, the viscosity increases at higher shear rates and the material is said to be shear thickening. These behaviours are depicted in Fig. 2.10 for the case of steady shear in figures that are usually referred to as flow curves. A flow curve is a rheogram with the shear stress as the ordinate and the applied shear rate as the abscissa.

The dynamic response of power-law fluids to oscillatory strain is shown in Fig. 2.11. Shear-thinning fluids, where $n<1$ dissipate more energy per unit volume per cycle as can be seen by the area their Lissajous curves enclose. It is common in the rheological literature to refer to the behaviours exhibited in Fig. 2.11 as strain-hardening (corresponding to $n>1)$ and strain-softening $(n<1)$ behaviours to discern between steady shear and oscillatory responses. These terms match the responses in that as the strain is increased, the stress increases faster for an $n>1$ material, a sign of the hardening of the material. For an $n<1$ material, the stress levels off with increasing strain, a sign of the material softening.

If $\eta \sim O\left(\eta_{\infty}\right)$ and $\eta \ll \eta_{0}$ such that $\eta_{0}-\eta \approx \eta_{0}$ then the Cross model reduces to

$$
\eta(\dot{\gamma})=\eta_{\infty}+K \dot{\gamma}^{n-1}
$$




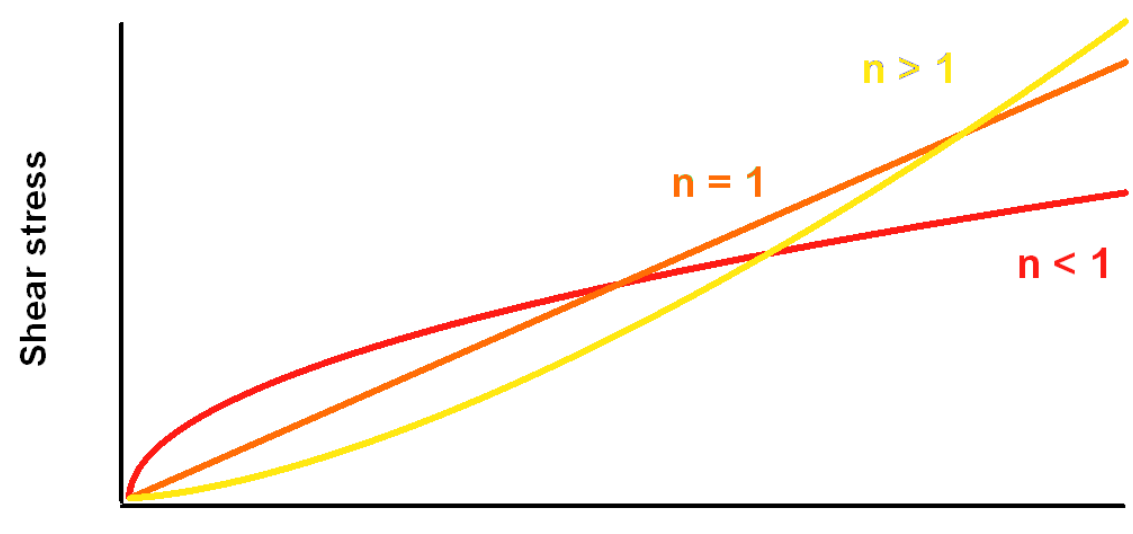

Shear rate

Figure 2.10: Flow curves showing Newtonian flow ( $n=1$, orange), shear-thinning $(n<1$, red) and shear-thickening ( $n>1$, yellow) behaviours.

which is known as the Sisko model. If $n=0$ in the Sisko model then the model reduces to

$$
\eta(\dot{\gamma})=\eta_{\infty}+K \dot{\gamma}^{-1}
$$

which is known as the Bingham model after the founder of the Society of Rheology. The stress defined by the Bingham model can then be written, with a change in parameters, as

$$
\sigma(\dot{\gamma})=\sigma_{y}+\eta_{p} \dot{\gamma}
$$

where $\sigma_{y}$ is referred to as the yield stress. The yield stress must be exceeded for the material to flow. The concept of a yield stress, though helpful in many circumstances, is strongly argued against on the basis that

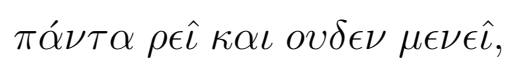

i.e. every material flows given enough time. If stresses below the yield stress are applied to a Bingham-type material the deformation achieved is purely elastic and will be recovered upon stress cessation. If a strain is applied instead of a stress to a Bingham material, the stress will rise until reaching the yield stress. Any strain acquired by the material after the yield stress has been reached will not be recovered upon cessation. This behaviour is known as plasticity and the deformation acquired after yielding is known as plastic deformation. 


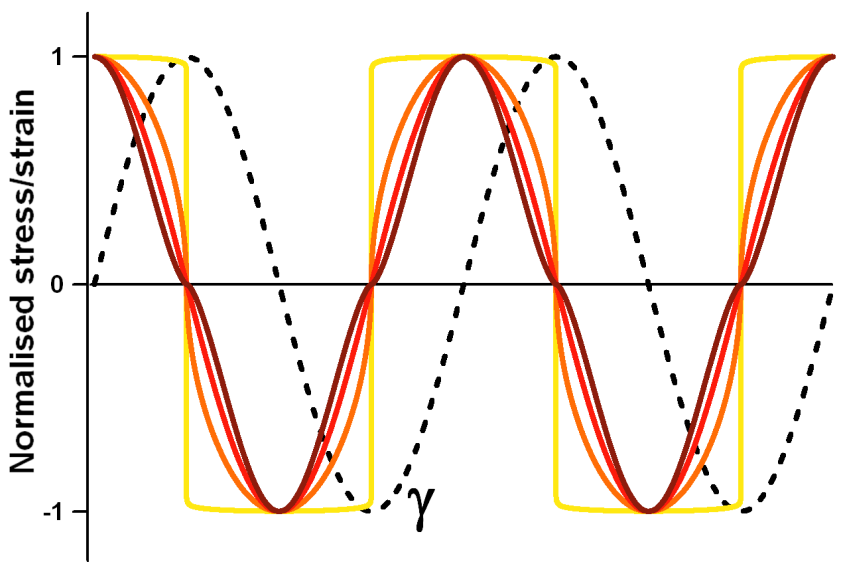

(a)

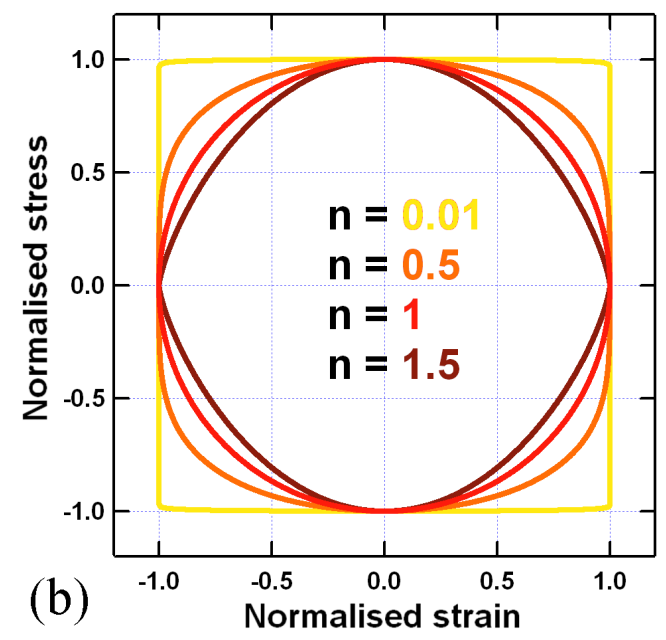

Figure 2.11: Dynamic response of power-law fluids for power-law indices (light to dark) $n=$ $0.01,0.5,1.0,1.5$. (a) Stress response (solid line) calculated from equation 2.38 compared with the strain input (dashed line) as functions of time. (b) Parametric Lissajous curves displaying the normalized stress as a function of the normalized strain for the same power-law indices.

\subsection{Rheometry}

The generic title of rheometry refers to the experimental techniques used to determine the rheological properties of materials and in this section we will introduce and discuss a few of the most common geometries for rotational devices. Rheometric devices that involve flow through constrictions or flow around obstructions, while important industrially and academically, will not be discussed. The simplest conceptual configuration of shear is that of two infinite, parallel plates separated by a distance $d$ in relative motion (see Fig. 2.2). This is generally called planar Couette flow after the French rheologist Maurice Marie Alfred Couette (1858 - 1943). While important theoretically, in practice this particular configuration is impossible to create and finite-sized approximations are inconvenient, so other configurations must be used in order to acquire rheological information.

Rotational rheometers involve two surfaces in relative rotational motion with the sample placed in the gap between them. The geometries we will discuss here are concentric-cylinder Couette in which a cylinder of radius $R_{I}$ is placed inside a cylinder with an inner radius of $R_{O}$ with the two cylinders sharing a common axis of rotation, cone-and-plate, in which a small-angle cone of radius $R_{0}$ and angle $\varphi_{0}$ is in relative- 
rotational motion a distance $h$ above a flat plate and plate-and-plate, in which two flat, circular plates of radius $R_{0}$ are a distance $h$ apart and in relative-rotational motion about a common axis.

\subsubsection{Geometries}

\section{Concentric-cylinder Couette geometry}

We will start our discussion of geometries with the concentric-cylinder Couette geometry shown schematically in Fig. 2.12. In the limit where the radii of both cylinders is $\infty$, this geometry reduces to the planar Couette configuration previously discussed. Practically, however, the radii are finite and the obvious coordinate system to work in is cylindrical coordinates shown also in Fig. 2.12. In cylindrical coordinates the velocity field that results from a relative rotational motion of $v_{\theta}(r)$ is

$$
\begin{aligned}
& v_{\theta}=v_{\theta}(r) \\
& v_{r}=0 \\
& v_{z}=0
\end{aligned}
$$

and the shear rate is equal to

$$
\dot{\gamma}_{r \theta}=-r \frac{\partial}{\partial r}\left(\frac{v_{\theta}(r)}{r}\right)
$$

If a Newtonian fluid is placed in the gap and the relative rotational velocity between the two cylinders is $\Omega_{0}$, then the limiting velocities are $v_{\theta}\left(r=R_{I}\right)=\Omega_{0} R_{I}$ and $v_{\theta}(r=$ $\left.R_{O}\right)=0$. This gives a velocity profile of

$$
v_{\theta}(r)=\frac{\Omega_{0} R_{I}^{2}}{R_{O}^{2}-R_{I}^{2}}\left[\frac{R_{O}^{2}}{r}-r\right] .
$$

And we can define a 'gap average' shear rate using the conventions set up in Fig. 2.2, that is taking the limit as the radius of curvature tends to infinity, as being

$$
\dot{\gamma}_{r \theta}=\frac{\Omega_{0} R_{I}}{R_{O}-R_{I}} .
$$

It should be noted that equation 2.45 merely defines the average shear rate over the gap and not the actual local shear rate. To obtain the exact local shear rate, equation 2.43 should be used. 


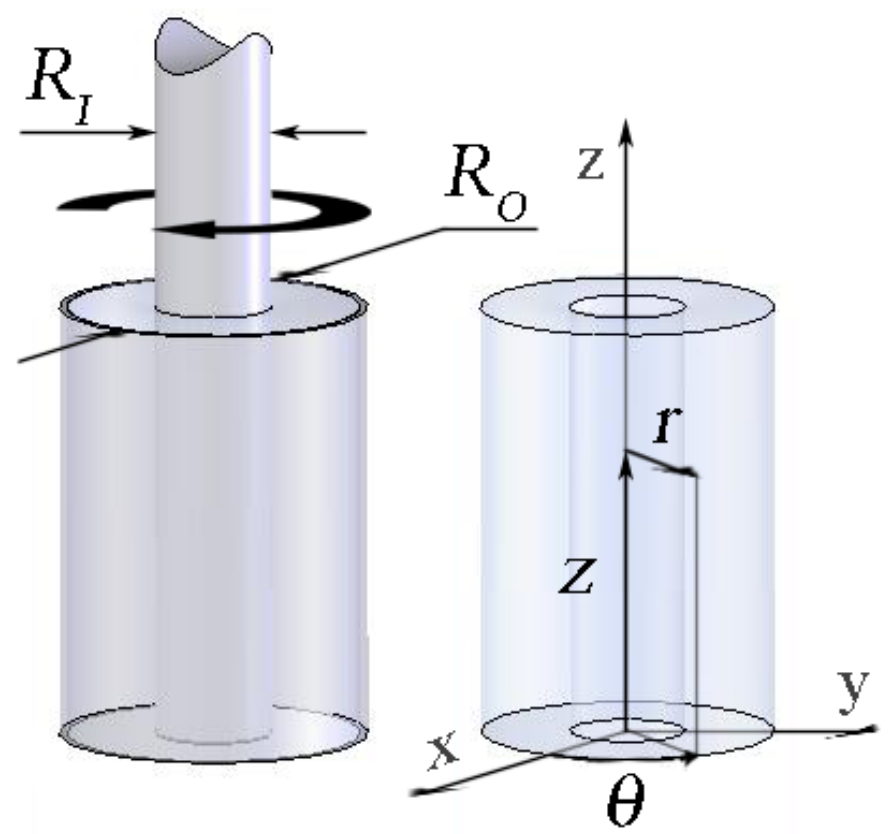

Figure 2.12: Concentric-cylinder Couette geometry: Cylinders of radius $R_{I}$ and $R_{O}$ are coaxial and are rotated with a relative-angular velocity. The sample to be tested is placed in the gap between the cylinders to form an annulus of height $H$. Cylindrical polar coordinates are used in the discussion of this geometry and are shown for reference.

If the torque exerted by the (Newtonian) material on the inner cylinder is denoted by $\Gamma$ and the cylinders are a height $H$, the viscosity can be expressed by

$$
\eta(\dot{\gamma})=\frac{\Gamma\left(R_{O}-R_{I}\right)}{2 \pi R_{I}^{3} L \Omega_{0}}
$$

Using equation 2.43 in conjunction with equations 2.44 and 2.36, we can see that the only non-zero component of the stress tensor is $\sigma_{r \theta}$ which can be conveniently written as

$$
\sigma_{r \theta}=\frac{C}{r^{2}}
$$

which tells us that the stress across the gap of a concentric-cylinder Couette geometry decays as $r^{-2}$. 


\section{Cone-and-Plate}

In the cone-and-plate geometry shown in Fig. 2.13, a small angle cone of angle $\varphi_{0}$ (the angle is typically only a few degrees and is exaggerated in Fig. 2.13 for visual purposes) and radius $R_{0}$ has its apex touching a coaxial flat plate. There is a relative-rotational motion about the common axis. Spherical polar coordinates are used to simplify the discussion of this geometry and a representation of these is also shown in Fig. 2.13.

If the cone is turned (we can always treat this as the case via a simple change of reference frame. The physics remains the same.) with an angular velocity of $\Omega_{0}$, then any part of the cone a distance $r$ from the cone apex will have a velocity $U=\Omega_{0} r$ and the distance between the cone and the plate can be written as $d=r \sin \varphi_{0}$, which, because of the small angle can be approximated as $d=r \varphi_{0}$. From this approximation we can say the velocity gradient i.e. the shear rate is $U / d$ which simplifies to

$$
\dot{\gamma}=\frac{\Omega_{0} r}{r \varphi_{0}}=\frac{\Omega_{0}}{\varphi_{0}}
$$

Equally, we can say that to a good approximation for a Newtonian fluid, the velocity profile will be

$$
\begin{aligned}
& v_{r}=0 \\
& v_{\varphi}=0 \\
& v_{\theta}=\Omega_{0} r\left(\frac{\pi / 2-\varphi}{\varphi_{0}}\right)
\end{aligned}
$$

and the only non-zero component of the shear rate tensor, $\dot{\gamma}_{\varphi \theta}$ is equal to

$$
\dot{\gamma}_{\varphi \theta}=\frac{\sin \varphi}{r} \frac{\partial}{\partial \varphi}\left(\frac{v_{\theta}}{\sin \varphi}\right) \doteq \frac{1}{r} \frac{\partial}{\partial \varphi} v_{\theta}=-\frac{\Omega_{0}}{\varphi_{0}}
$$

which is the same as equation 2.48 .

This result is important because it makes clear that the shear rate is the same everywhere in the gap, providing the angle of the cone is small enough for $\sin \varphi$ to be approximated by 1 .

Because the shear rate is the same everywhere in the gap, the stress also will be the same everywhere in the gap. Calculating the non-zero component of the stress tensor, $\sigma_{\varphi \theta}$, is done by working out the torque, $\Gamma$, required to keep a constant motion. This is done by integrating the product of the force $\left.\sigma_{\varphi \theta}\right|_{\varphi=\pi / 2} r d r d \theta$ and the lever arm $r$ over 

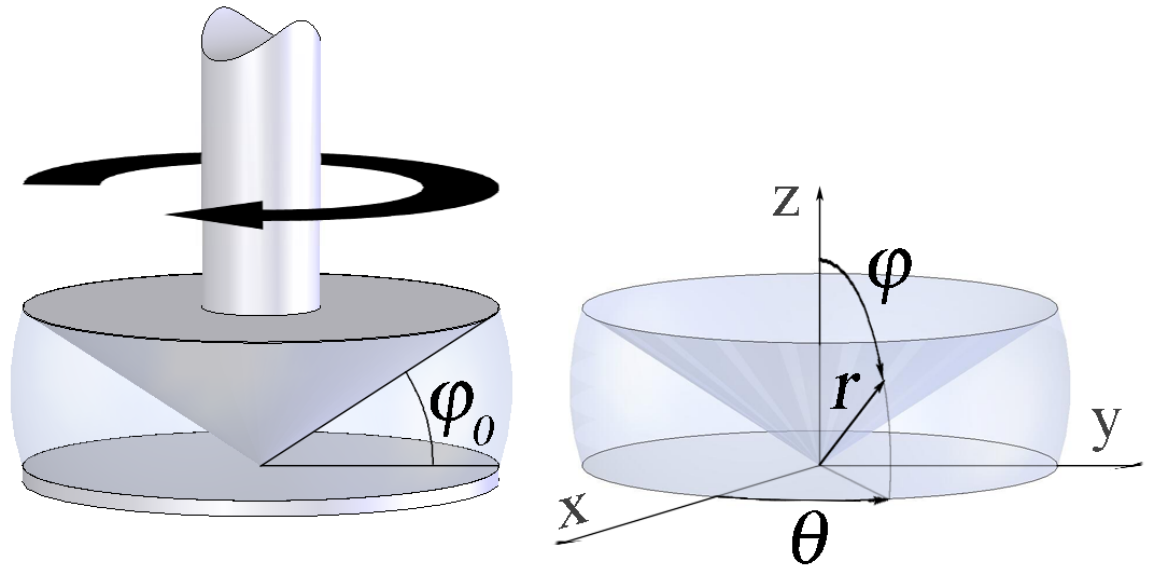

Figure 2.13: Cone-and-plate geometry: A cone of angle $\varphi_{0}$ (here greatly exaggerated for visual purposes) and radius $R$ is coaxial to a plate typically of radius $R_{\text {plate }}=R_{\text {cone }}$ or $R_{\text {plate }}>>R_{\text {cone }}$ and rotated with a relative angular velocity. The sample to be tested is placed in the gap between the two. Spherical polar coordinates are used in the discussion of this geometry and are shown for reference.

the surface of the plate

$$
\Gamma=\left.\int_{0}^{2 \pi} \int_{0}^{R} \sigma_{\varphi \theta}\right|_{\varphi=\pi / 2} r^{2} d r d \theta
$$

which can be performed easily and rearranged to give

$$
\sigma_{\varphi \theta}=\frac{3 \Gamma}{2 \pi R^{3}}
$$

Combining equations 2.50 and 2.52 we can express the viscosity of a Newtonian fluid in the gap of a cone and plate geometry as

$$
\eta=\frac{\sigma_{\varphi \theta}}{\dot{\gamma}_{\varphi \theta}}=\frac{3 \Gamma \varphi_{0}}{2 \pi R^{3} \Omega_{0}}
$$

\section{Plate-and-Plate}

In the plate-and-plate geometry shown in Fig. 2.14, two coaxial circular plates, radius $R_{0}$, are separated by a distance $h$. There is a relative-rotational motion about the common axis. Cylindrical polar coordinates are used to simplify the discussion of this geometry and a representation of these is also shown in Fig. 2.14. 
The velocity field set up by a relative rotational velocity of $\Omega_{0}$ in a Newtonian fluid is

$$
\begin{aligned}
v_{r} & =0 \\
v_{\theta}(r, z) & =\frac{\Omega_{0} r z}{h} \\
v_{z} & =0
\end{aligned}
$$

and the shear rate is equal to

$$
\begin{aligned}
& \dot{\gamma}_{r \theta}=-r \frac{\partial}{\partial r}\left(\frac{v_{\theta}(r)}{r}\right) \\
& \dot{\gamma}_{z \theta}=\frac{1}{r} \frac{\partial v_{z}}{\partial \theta}+\frac{\partial v_{\theta}}{\partial z}
\end{aligned}
$$

which means the only non-zero component is

$$
\dot{\gamma}_{z \theta}=\frac{\Omega r}{h}
$$

and by extension the stress tensor's only non-zero component is

$$
\sigma_{z \theta}=-\frac{\eta \Omega_{0} r}{h}
$$

The torque exerted on the stationary plate can again be calculated by integrating the product of the shear stress $\sigma_{z \theta}$ and the lever arm $r$ over the differential surface area of the plate, $2 \pi r d r$ :

$$
\Gamma=\int_{0}^{R}-\sigma_{z \theta} 2 \pi r^{2} d r=-\frac{\eta \Omega_{0} 2 \pi}{h} \int_{0}^{R} r^{3} d r=-\frac{\eta \Omega_{0} \pi R^{4}}{2 h}
$$

\subsubsection{Slip}

The results derived in the previous section all assume that when liquid flows over a solid surface, the liquid molecules adjacent to the solid are stationary relative to the solid. This is known as the Non-Slip Boundary Condition (NSBC). When this assumption does not hold and slip occurs, there is a non-zero relative velocity. Navier [7] defined the 'slip length' $b$, characterising the slip boundary condition in 1827 . For flow adjacent to a planar, impermeable solid surface he defined the slip length by

$$
v_{\| 0}(t)=\left.b \frac{\partial v_{\|}(z, t)}{\partial z}\right|_{z=0}
$$




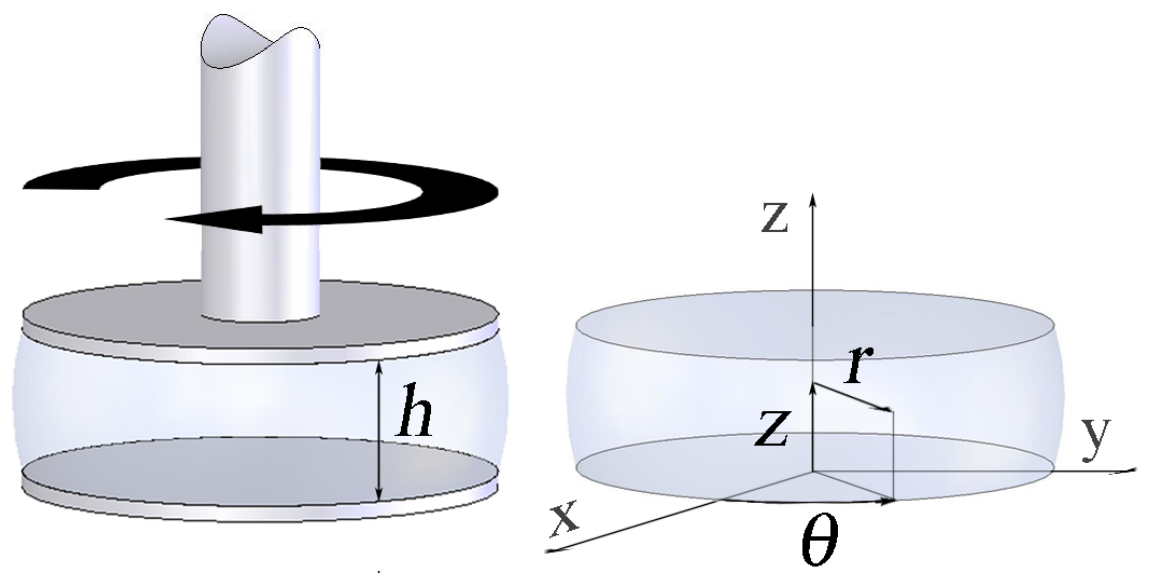

Figure 2.14: Plate-and-plate geometry: Two coaxial plates of radius $R$ are separated by a distance $h$ and have a relative rotational velocity about their common axis. The sample to be tested is placed in the gap between the two. Cylindrical polar coordinates are used in the discussion of this geometry and are shown for reference.

where $v_{\|}$is the shear velocity relative to and parallel with the shearing surface and $v_{\| 0}$ is the value of $v_{\|}$at $z=0$. This effectively defines a distance $b$ into the shearing surface where the velocity would be expected to be zero. A visualisation of this definition is presented in Fig. 2.15. The difference between the NSBC shown in Fig. 2.2 and the slip shown in Fig. 2.15 is clear.

For a long period of time following the work of Navier, the NSBC was an adequate boundary condition for the study of both Newtonian and non-Newtonian fluids. In the second half of the 20th century, however, many investigations into non-Newtonian polymer solutions showed the existence of slip with slip lengths on the order of $\sim \mu m$. See, for example [8]. Recent work on Newtonian fluids has shown slip lengths on the order of $10-100 \mathrm{~nm}[9,10]$.

A common approach to reducing the effect of slip is to increase the roughness of the shearing surface, thus increasing the surface area between the fluid and the solid surface.

The existence, or lack, of slip must be foremost in the mind of the experimenter when investigating the viscoelastic regime of non-Newtonian fluids. Spurious results can often be explained by the existence of slip. 


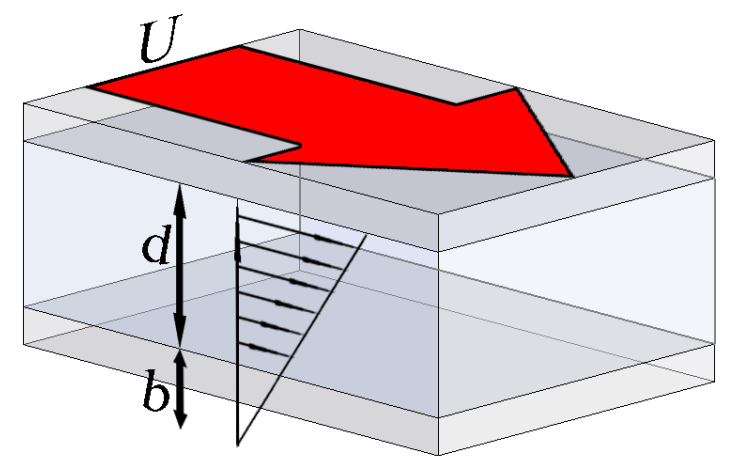

Figure 2.15: Flow with non-zero slip. The 'slip length', $b$, is defined as being the distance into the shearing surface where the velocity would be expected to be zero. 


\section{Chapter 3}

\section{Nuclear Magnetic Resonance (NMR)}

Nuclear magnetic resonance, or NMR, is a physical phenomenon that is based on the interaction between the magnetic moments of nuclei and magnetic fields. The physics that describes this phenomenon is quantum mechanical and relies on the idea of quantisation at the nuclear level. NMR experiments are carried out on large numbers of particles simultaneously, referred to as an ensemble, which means the discrete values possible in the quantum regime manifest themselves as continuous macroscopic relations that can be described semi-classically. This chapter will introduce the quantum mechanics necessary to explain the phenomenon of NMR and then adopt a semi-classical approach in dealing with some of the key experimental details used later in this thesis. For some comprehensive texts on all areas of NMR see [11, 12, 13, 14, 15, 16, 17].

\subsection{Nuclear spin and magnetic moment}

Atomic nuclei are comprised of combinations of protons and neutrons which have intrinsic angular momentum called spin. Spin, like charge and mass, is a fundamental property of nature. Protons and neutrons, collectively referred to as nucleons, are both fermions and as such have spin $\hbar / 2$, where $\hbar$ is Planck's constant divided by $2 \pi$ (also known as Planck's reduced constant). To make the rest of this discussion simpler, we shall adopt a system of units such that $\hbar=1$ so that angular momentum will be expressed as numbers with the assumption of units of $\hbar$. Under this system protons and neutrons can be called spin- $1 / 2$ particles. Their fermionic nature makes them susceptible to Pauli's exclusion principle, just as are electrons. When protons and neutrons 
group together to form atomic nuclei, their individual spins act to give the nucleus a spin of its own, characterised by the spin quantum number $I$. There is evidence [18] that nucleons form a shell structure in nuclei similar to the orbital-filling manner of electrons, a behaviour that is captured in a theory known as the nuclear shell model. The pairing hypothesis is an important idea that suggests that nucleons, unlike electrons, pair up even when a shell is unfilled. This limits the number of unpaired nucleons and accounts for the spin properties of ground-state nuclei, some of which are listed in Table 3.1.

Table 3.1: Some nuclei that have spin quantum numbers, $I$

\begin{tabular}{cc}
\hline$I$ & Nuclei (Spin value) \\
\hline \hline 0 & ${ }^{12} \mathrm{C},{ }^{16} \mathrm{O},{ }^{32} \mathrm{~S}$ \\
& ${ }^{1} \mathrm{H},{ }^{3} \mathrm{H},{ }^{13} \mathrm{C}$, \\
$1 / 2$ & ${ }^{15} \mathrm{~N},{ }^{19} \mathrm{~F},{ }^{31} \mathrm{P}$ \\
& ${ }^{2} \mathrm{H},{ }^{14} \mathrm{~N}$ \\
1 & \\
$>1$ & ${ }^{11} \mathrm{~B}(3 / 2),{ }^{23} \mathrm{Na}(3 / 2)$ \\
& ${ }^{17} \mathrm{O}(5 / 2),{ }^{10} \mathrm{~B}(3)$ \\
\hline
\end{tabular}

The magnetic dipole moment of a nucleus, $\boldsymbol{\mu}$, is proportional to the angular momentum I with the proportionality constant, $\gamma$, known as the gyromagnetic ratio. Note that the angular momentum associated with nuclear spin is a vector and is written in bold, whereas the scalar spin quantum number, $I$, is not. Formally,

$$
\boldsymbol{\mu}=\gamma \boldsymbol{I}
$$

The gyromagnetic ratio is a property of the particular nucleus and in the case of protons is equal to $2.675 \times 10^{8} \mathrm{rad} \mathrm{s}^{-1} T^{-1}$. Only nuclei with $I \neq 0$ (that is to say, nuclei with unpaired protons or neutrons) concern us because only nuclei with non-zero spin have non-zero magnetic moments and hence are NMR-active.

The angular momentum, I, associated with nuclear spin and the corresponding magnetic moment, $\mu$ are quantised. The eigenstates, or energy levels corresponding to the quansitsation, are characterised by the magnetic quantum number $m$. The eigenvalues of the angular momentum along the z-axis, $I_{z}$ are given by

$$
I_{z}=m
$$


and the z-component of the magnetic moment is

$$
\mu_{z}=\gamma I_{z}=\gamma m
$$

The magnetic quantum number $m$ can take any integer value between $I$ and $-I$, meaning there are $2 I+1$ distinct angular momentum states. For a nucleus with $I=1 / 2$ there are thus two distinct angular momentum states that, in the absence of an external magnetic field, are at the same energy and will be populated by exactly the same number of nuclei at thermal equilibrium. Such states are said to be degenerate.

\subsection{Zeeman interaction}

When nuclei with non-zero spin, and hence non-zero magnetic moments, are placed in a magnetic field, $\mathbf{B}_{0}$, they experience torques which tend to align them with the direction of the field. The classical and quantum mechanical interaction energy is given by the Hamiltonian

$$
\mathbf{H}=-\mu \cdot \mathbf{B}_{0} .
$$

If we use the direction of the magnetic field to define the z-axis, we can write the Hamiltonian as

$$
\mathrm{H}=-\gamma B_{0} I_{z}
$$

This form of the Hamiltonian is known as the Zeeman interaction after Dutch physicist Pieter Zeeman (May 25, 1865 - October 9, 1943) who won the Nobel Prize for physics along with Hendrik Lorentz in 1902 for its discovery. As a result of this relation, different spin states will have different energies. The energy associated with these different spin states differs by an amount $\Delta E=\gamma \hbar B_{0}$ that is linearly proportional to the strength of the magnetic field as shown in Fig. 3.1 for a spin 1/2 system. Transitions between energy levels, as shown in Fig. 3.1 can be induced when photons with energies that match the difference between levels are absorbed. The energy of a photon is expressed as $E_{\text {photon }}=h \nu$ where $h$ is Planck's constant and $\nu$ is the frequency. The frequency required to cause a transition from the lower energy state to the higher energy state is therefore

$$
\nu_{0}=\frac{E}{h}=\frac{\gamma B_{0}}{2 \pi}
$$

which gives an angular frequency of

$$
\omega_{0}=2 \pi \nu_{0}=\gamma B_{0} .
$$




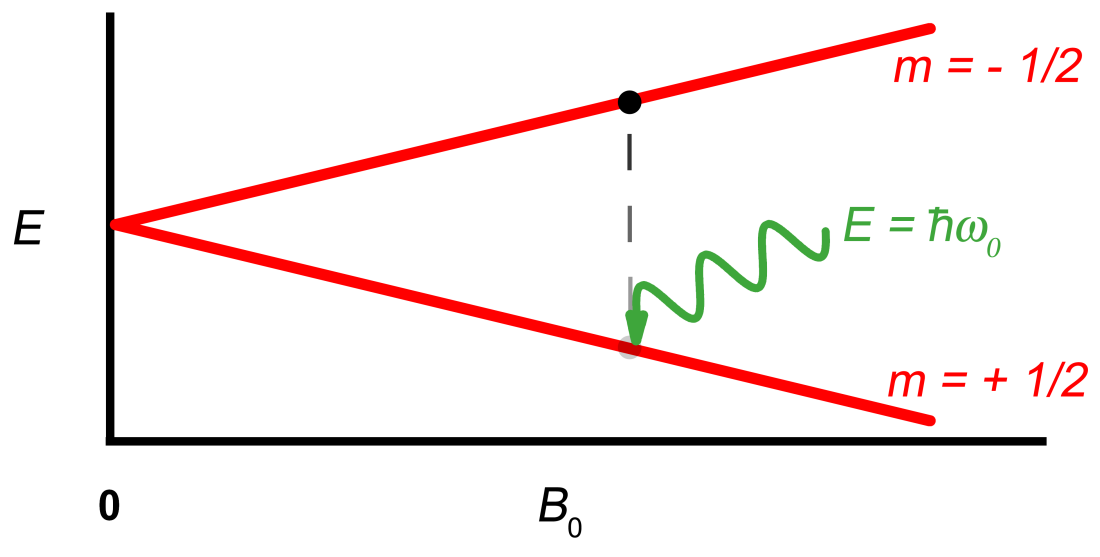

Figure 3.1: The application of a magnetic field splits the energy levels of a spin $1 / 2$ system by an amount $\gamma \hbar B_{0}$. Transitions from the lower energy state to the higher energy state can be induced via the absorption of a photon at the Larmor frequency $\omega_{0}$.

This is known as the Larmor frequency, named after the Irish physicist Joseph Larmor (11 July 1857 - 19 May 1942).

When a nucleus with a non-zero magnetic moment is placed in a magnetic field, the torque exerted on it is additional to an already existing angular momentum. This results in the nucleus precessing about the magnetic field at a characteristic frequency, the Larmor frequency. We can also understand this phenomenon in terms of the evolution operator, which is defined as

$$
U(t)=\exp (-i H t / \hbar)
$$

which, in the present case is $U(t)=\exp \left(i \gamma B_{0} I_{z} t\right)$. This is equivalent to a clockwise rotation about the $\mathrm{z}$ axis by an angle $\gamma B_{0} t$. The presence of the field thus causes all the states to precess at the Larmor frequency, $\omega_{0}$, given by

$$
\omega_{0}=\gamma B_{0} .
$$

This is the same result as equation 3.7.

For a large laboratory superconducting magnet with a $B_{0}$ field of 9.4 Tesla, the precession frequency of a proton is $400 \mathrm{MHz}$, which corresponds to the UHF part of the radio-frequency spectrum. 


\subsection{Other interactions}

The largest nuclear interaction in the high-field case is the coupling of the nuclear magnetic moment to an external field, the Zeeman interaction previously discussed. Other weaker interactions exist when spins interact with each other and when spins interact with additional local and applied magnetic fields. The total Hamiltonian of a spin system is the sum of the operators for each interaction such that the total Hamiltonian may be expressed as

$$
\mathbf{H}=\mathbf{H}_{Z}+\mathbf{H}_{Q}+\mathbf{H}_{r f}+\mathbf{H}_{D}+\mathbf{H}_{C S}+\mathbf{H}_{J}
$$

This expression lists the interactions in decreasing strength so that the Hamiltonian for the Zeeman interaction, $\mathbf{H}_{Z}$ is listed before the Hamiltonians for the quadrupolar interaction, $\mathbf{H}_{Q}$, the coupling of the spins to the exciting r.f. field, $\mathbf{H}_{r f}$ (which takes the same form as the Zeeman interaction for the applied $\mathbf{B}_{1}$ r.f. field and so will not be discussed further), the dipolar coupling, $\mathbf{H}_{D}$, the chemical shift, $\mathbf{H}_{C S}$, and the indirect coupling $\mathbf{H}_{J}$. The energy levels of the system are the eigenvalues of the Hamiltonian. This section will provide an elementary introduction of the interactions relevant to this thesis. A more complete discussion of nuclear interactions may be found in any standard NMR text such as Abragam [11].

\subsubsection{The quadrupolar interaction}

Nuclei that possess spin greater than $\frac{1}{2}$ are non-spherical and hence have electric quadrupole moments that interact with the electric field gradients established by the electrons surrounding them. In the laboratory frame, in the high-field limit, the Hamiltonian for the quadrupolar interaction takes the form [11]

$$
\mathbf{H}_{Q}=\frac{e^{2} q Q}{8 I(2 I-1)}\left[3 I_{z}^{2}-I(I+1)\right]\left[\left(3 \cos ^{2} \theta-1\right)+\eta \sin ^{2} \theta \cos (2 \phi)\right]
$$

where $\theta$ and $\phi$ are the polar and azimuthal angles of $\mathbf{B}_{0}$ in the principal axis system of the electric field gradient, $\eta$ is the asymmetry parameter defined as $\frac{V_{x x}-V_{y y}}{V_{z z}}$ where $V_{\alpha \beta}=\frac{\partial^{2} V}{\partial \alpha \partial \beta}, \alpha, \beta=x, y, z$ with $\mathbf{V}=V_{\alpha \beta}$ being the electric field gradient tensor, and $Q$ is the nuclear quadrupole moment, which is a property of the nucleus. The quadrupole moment, $Q$ is positive for prolate (relative to the field direction) nuclei and negative for oblate nuclei. For a system of nuclei with $I=1$ in an axially symmetric electric field 
(making the asymmetry parameter $\eta$ equal to zero), the Hamiltonian can be rewritten as

$$
\mathbf{H}_{Q}=\frac{e^{2} q Q}{8}\left(3 I_{z}^{2}-2\right)\left(3 \cos ^{2} \theta-1\right) .
$$

If the only interactions taken into account are the Zeeman and quadrupolar interactions, the total Hamiltonian of the system has just two components:

$$
\mathbf{H}=\mathbf{H}_{Z}+\mathbf{H}_{Q}
$$

that has eigen-values to first order of

$$
E_{m_{I}}=\gamma B_{0} m_{I}+\left(3 m_{I}^{2}-2\right) \frac{e^{2} q Q}{8}\left(3 \cos ^{2} \theta-1\right)
$$

where we note that for a system with spin $I=1$, the magnetic quantum number can take values of $(-1,0,1)$. Such a system of energy levels is illustrated in Fig. 3.2. From equation 3.14 it can be seen that we obtain two resonance lines at $\omega_{0} \pm \omega^{\prime}$ where $\omega^{\prime}=$ $\frac{3 e^{2} q Q}{8}\left(3 \cos ^{2} \theta-1\right)$, such that the splitting, $\Delta \omega=2 \omega^{\prime}$ is equal to

$$
\Delta \omega=\frac{3 e^{2} q Q}{4}\left(3 \cos ^{2} \theta-1\right) .
$$

The $\theta$-dependence on the splitting is an important feature of equation 3.15 worth making note of. For nuclei with spin $I \geq 1$ in a rigid lattice, the quadrupolar interaction disappears when $\cos \theta=1 / \sqrt{3}$, leading to a critical angle for which no quadrupolar effects are seen, allowing measurements of weaker interactions. In liquids, the random molecular tumbling tends to average the quadrupolar term out to zero, unless there is physical structure present. This means the quadrupole interaction is important in determining alignment in liquids.

\subsubsection{The dipolar interaction}

The dipolar interaction occurs between magnetic moments associated with individual spins in a system. For two like dipoles (associated with like nuclei with identical values for $\gamma$ ), $\mathbf{I}_{1}$ and $\mathbf{I}_{2}$, separated by a vector $\mathbf{r}$, the Hamiltonian for this dipole-dipole interaction can be written as

$$
\mathbf{H}_{D}=\frac{\gamma^{2} \hbar^{2}}{r^{3}} \mathbf{I}_{1} \cdot \mathbf{I}_{2}-\frac{3\left(\mathbf{I}_{1} \cdot \mathbf{r}\right)\left(\mathbf{I}_{2} \cdot \mathbf{r}\right)}{r^{2}}
$$

Generally, the fast molecular tumbling associated with liquids causes the dipolar interaction to average to zero. 


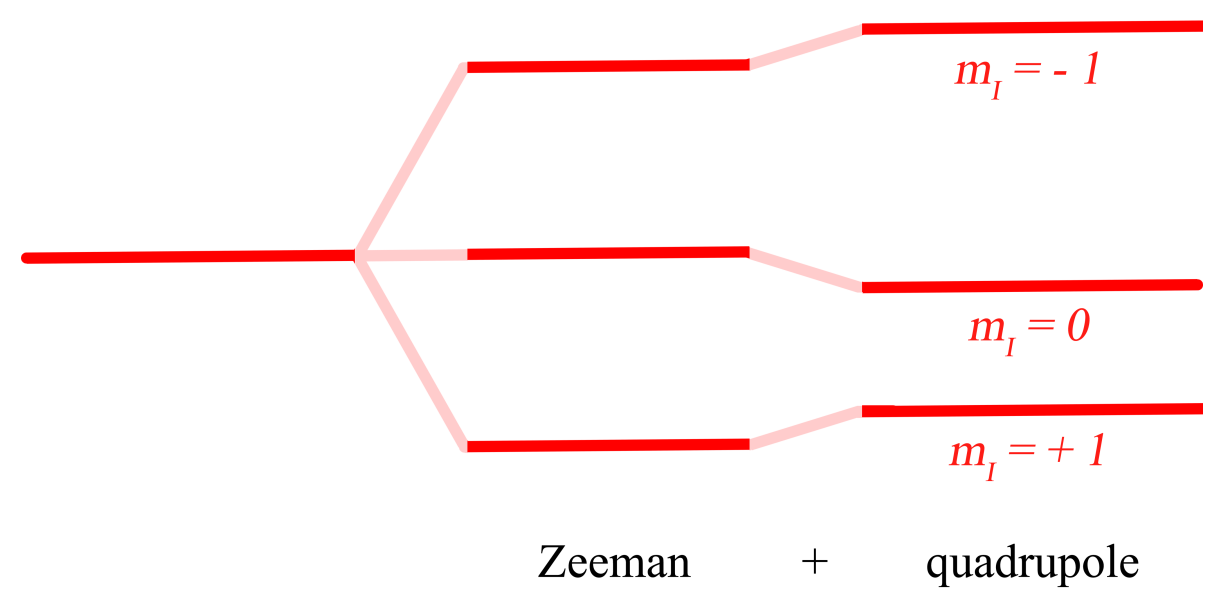

Figure 3.2: The presence of a quadrupole interaction for nuclei with spin $I \geq 1$ alters the energy levels, and hence the splitting, caused by the Zeeman interaction.

\subsubsection{Chemical shift}

Electrons orbiting nuclei in an external field act to alter the strength of the field experienced at the nucleus. This shielding is characteristic of the local electronic environment and causes shifts in the Larmor frequency at the nuclei which depend on the local chemistry. The chemical shift, as it is known, can provide a spectral fingerprint of a given molecule, aiding in the determination of the structure. The discovery of the chemical shift in the 1950s changed chemistry forever. The Hamiltonian for this interaction is

$$
\mathbf{H}_{C S}=-\mathbf{I} \cdot \mathbf{S} \cdot \mathbf{B}_{0}
$$

where $\mathbf{S}$ is known as the chemical shift tensor or shielding tensor. The off-diagonal terms of $\mathbf{S}$ are zero for fluids due to averaging by molecular tumbling. Chemical shifts, $\delta$, are normally expressed in units of parts per million (p.p.m.) which are calculated as

$$
\delta=\frac{\text { frequency of signal - frequency of reference }}{\text { frequency of spectrometer (in } \mathrm{MHz})} .
$$

The reference signal used to calculate chemical shifts for ${ }^{1} \mathrm{H},{ }^{13} \mathrm{C}$, and ${ }^{29} \mathrm{Si}$ nuclei is tetramethylsilane (TMS) which is ascribed a chemical shift of 0 . 


\subsection{Bulk Magnetisation}

In the absence of a magnetic field, the energy levels of a non-zero spin system are degenerate and are populated by an equal number of nuclei. We have, in the preceding section, seen that a magnetic field splits those energies via the Zeeman interaction causing one state to be energetically more favourable than the others as in Fig. 3.1. How these states are populated at thermal equilibrium in a magnetic field is explained through the use of Boltzmann statistics.

We will treat the case of $I=1 / 2$ where there are two distinct energy states and denote the population of the $m=+1 / 2$ by $n_{+}$and the population of the $m=-1 / 2$ state by $n_{-}$. The spins in the $m=+1 / 2$ state can be thought of as aligning parallel and the spins in the $m=-1 / 2$ state antiparallel to the field. At thermal equilibrium at some temperature $T>0 K$, the number of spins in the lower energy $m=+1 / 2$ state, $n_{+}$, will slightly outnumber the number of spins in the higher energy $m=-1 / 2$ state, $n_{-}$, and the relative populations can be expressed as

$$
\frac{n_{-}}{n_{+}}=\exp \frac{-\Delta E}{k_{B} T}
$$

where $\Delta E$ is the difference in energy between the states, $k_{B}$ is Boltzmann's constant and has a value of $1.3805 \times 10^{-23} \mathrm{~J} \mathrm{~K}^{-1}$ and $T$ is the temperature measured in Kelvin. The population difference can be worked out using the fact that all spins, $N$, are accounted for by $n_{+}+n_{-}$, using equation 3.19:

$$
n_{+}-n_{-}=N \frac{1-\exp \left(-\gamma \hbar B_{0} / k_{B} T\right)}{1+\exp \left(-\gamma \hbar B_{0} / k_{B} T\right)}
$$

Expanding equation 3.20 using the Taylor series for $\exp (-x)$ yields

$$
n_{+}-n_{-}=N \frac{1-\left(1-\gamma \hbar B_{0} / k_{B} T+\gamma^{2} \hbar^{2} B_{0}^{2} / 2 k_{B}^{2} T^{2}-\ldots\right)}{1+\left(1-\gamma \hbar B_{0} / k_{B} T+\gamma^{2} \hbar^{2} B_{0}^{2} / 2 k_{B}^{2} T^{2}-\ldots\right)} .
$$

At room temperature, $k_{B} T>>\hbar B_{0}$ and so the squared and higher terms can be neglected to give

$$
n_{+}-n_{-} \approx \frac{N \gamma \hbar B_{0} / k_{B} T}{2-\gamma \hbar B_{0} / k_{B} T}
$$

Again we make the note that at room temperature $2>\gamma \hbar B_{0} / k_{B} T$ and so we can simplify the above to give

$$
n_{+}-n_{-} \approx \frac{N \gamma \hbar B_{0}}{2 k_{B} T}
$$


The magnetisation of the bulk, $M_{0}$, is found by multiplying the spin population difference by the magnetic moment from equation 3.3:

$$
M_{0}=\left(n_{+}-n_{-}\right) \gamma \hbar m \approx \frac{N \gamma^{2} \hbar^{2} B_{0}}{4 k_{B} T}=\frac{C B_{0}}{T}
$$

This is known as Curie's law. It relates the temperature-dependent paramagnetism, $M_{0}$, to the magnetic field, $B_{0}$ and the material-dependent Curie constant. Curie's law is a high-temperature approximation to Langevin's paramagnetic relation

$$
M_{0}=N \mu\left[\operatorname{coth}\left(\frac{\mu B_{0}}{k_{B} T}\right)-\frac{k_{B} T}{\mu B}\right]
$$

Using equation 3.22, often referred to as the Curie regime of equation 3.23, we can see that higher temperatures lead to a reduction in paramagnetism. This can be understood as thermal agitations reducing the amount of alignment of individual spins with the field, thereby reducing the paramagnetism of the bulk.

The fundamental result of this section is that for a spin- $1 / 2$ system at any finite temperature, there is an abundance of spins parallel compared with antiparallel to the field that results in a net magnetisation in the direction of the magnetic field. This magnetisation is a vector, $\mathbf{M}_{0}$ and is displayed in Fig. 3.3 lying in the same direction as both $\mathbf{B}_{0}$ and the $\mathrm{z}$ axis.

\subsection{Semi-classical description}

The motion of an ensemble of spin(1/2)s can be described in terms of what happens to the magnetisation vector $\mathbf{M}$. The macroscopic angular momentum vector is simply $\mathbf{M} / \gamma$, where $\gamma$ is the gyromagnetic ratio of the nuclei in question. When a magnetic moment is placed in a magnetic field, it experiences a torque equal to the cross product of the magnetisation and field vectors, $\mathbf{M} \times \mathbf{B}$. This is equal to the rate of change of angular momentum so that

$$
\frac{\mathrm{d} \mathbf{M}}{\mathrm{d} t}=\gamma \mathbf{M} \times \mathbf{B} .
$$

When the magnetic field is oriented in the $+z$ direction with magnitude $B_{0}$, as we have previously defined, the solution to equation 3.24 can be expressed in component form 


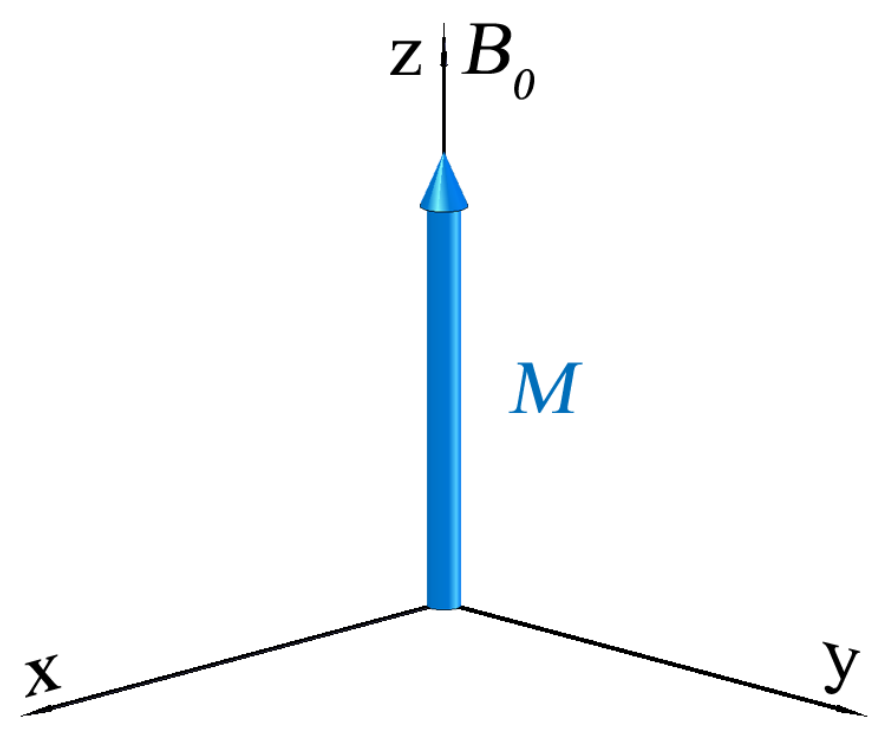

Figure 3.3: A bulk magnetisation results from a population difference between the upper and lower levels in a spin- $1 / 2$ system. It is represented by a vector $\mathbf{M}_{0}$ that lies in the same direction as $\mathbf{B}_{0}$ and the $\mathrm{z}$ axis at thermal equilibrium.

as

$$
\begin{aligned}
M_{x}(t) & \propto M_{0} \sin \omega_{0} t \\
M_{y}(t) & \propto M_{0} \cos \omega_{0} t \\
M_{z}(t) & \propto M_{0}
\end{aligned}
$$

which describes the precession of the magnetisation about the $\mathrm{z}$ axis at the Larmor frequency, $\gamma B_{0}$ as shown in Fig. 3.4.

\subsubsection{Excitation and the rotating frame}

Spins can be disturbed from their equilibrium states via the absorption of a Larmorfrequency photon. These photons are added to the system via the application of a linearly-polarised oscillating magnetic field of amplitude $B_{1}$ whose orientation is transverse to $\mathbf{B}_{0}$. It is conventional to represent this linearly polarised field as a sum of two counter-rotating circularly polarised components of magnitude $B_{1}$ as shown in Fig. 3.5. One of these components will rotate in the same direction as the nuclear precession and 


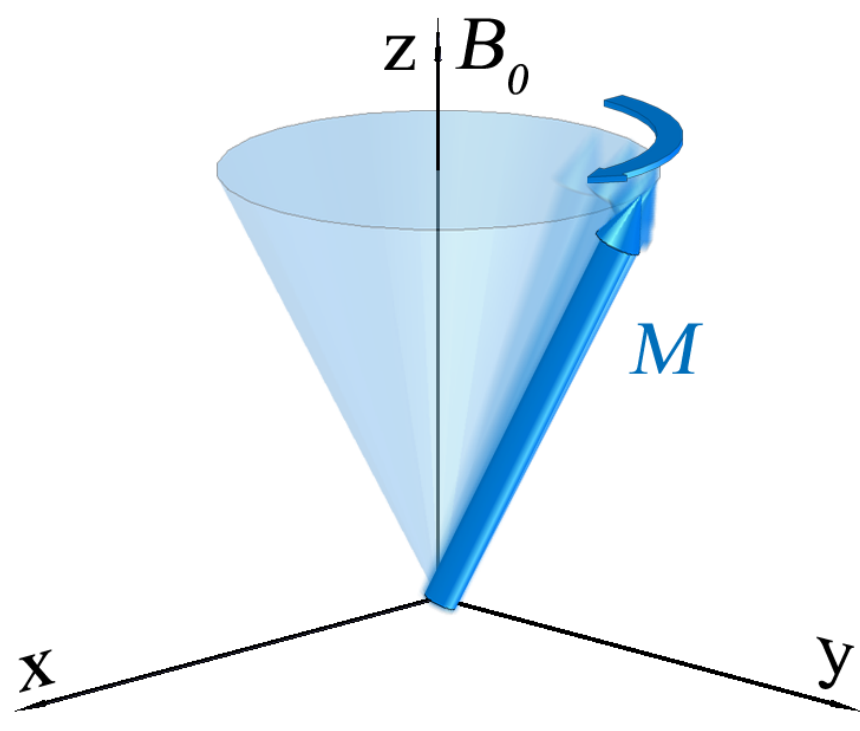

Figure 3.4: The bulk magnetisation vector $\mathbf{M}$ precesses about the $\mathrm{z}$ axis in the presence of a static magnetic field at the Larmor frequency.

the other will rotate in the opposite sense. We can neglect the counter-rotating component providing $B_{0}>>B_{1}$ which will invariably be the case. The component rotating with the nuclear spin precession will be responsible for the resonance phenomenon when the frequency of rotation is equal to the Larmor frequency, that is when $\omega=\omega_{0}$. We can write this vector as

$$
\mathbf{B}_{1}(t)=B_{1} \cos \omega t \mathbf{i}-B_{1} \sin \omega t \mathbf{j}
$$

where $\mathbf{i}, \mathbf{j}$ and $\mathbf{k}$ are the unit vectors in the $x, y$ and $z$ directions. Equation 3.24 then gives component-separated equations of

$$
\begin{aligned}
\frac{\mathrm{d} M_{x}}{\mathrm{~d} t} & =\gamma\left[M_{y} B_{0}+M_{z} B_{1} \sin \omega_{0} t\right] \\
\frac{\mathrm{d} M_{y}}{\mathrm{~d} t} & =\gamma\left[M_{z} B_{1} \cos \omega_{0} t+M_{x} B_{0}\right] \\
\frac{\mathrm{d} M_{z}}{\mathrm{~d} t} & =\gamma\left[-M_{x} B_{1} \sin \omega_{0} t-M_{y} B_{1} \cos \omega_{0} t\right],
\end{aligned}
$$




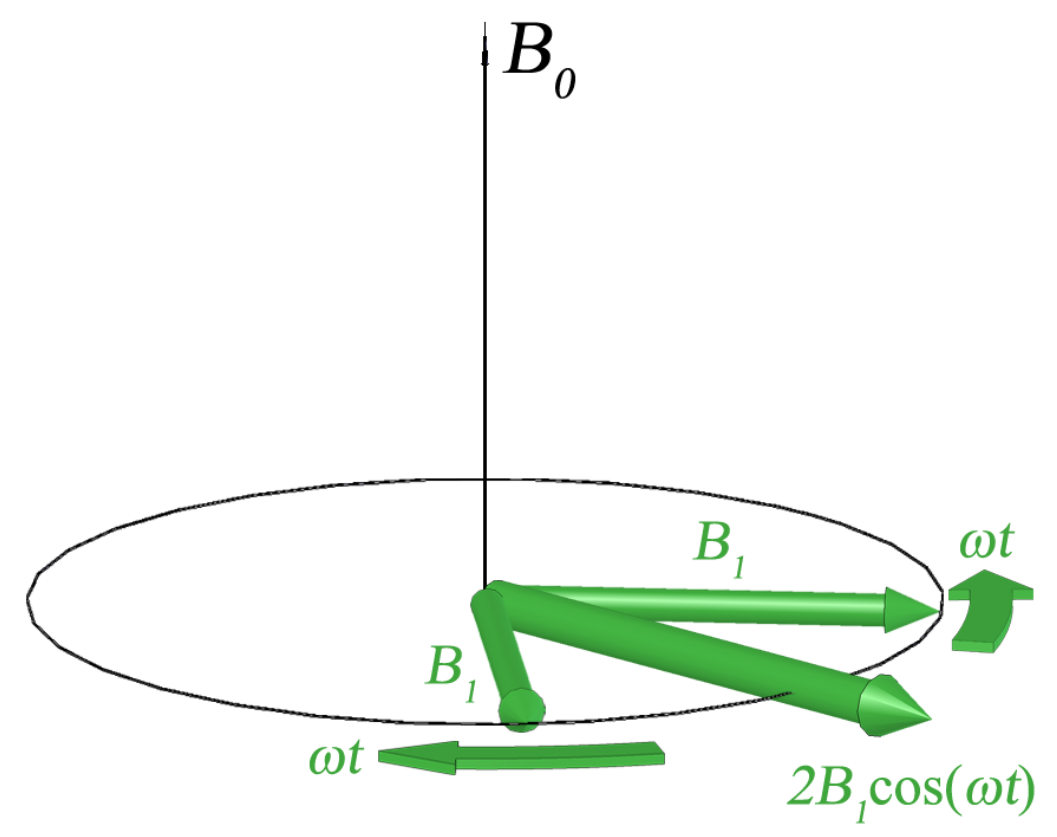

Figure 3.5: It is conventional to represent the linearly-polarised oscillating field (large vector) as a sum of two counter-rotating circularly polarised components of magnitude $B_{1}$ (small vectors).

that, given the initial condition of $\mathbf{M}(0)=M_{0} \mathbf{k}$ have solutions

$$
\begin{aligned}
& M_{x}=M_{0} \sin \omega_{1} t \sin \omega_{0} t \\
& M_{y}=M_{0} \sin \omega_{1} t \cos \omega_{0} t \\
& M_{z}=M_{0} \cos \omega_{1} t
\end{aligned}
$$

where $\omega_{1}=\gamma B_{1}$. Equations 3.28 imply that under the influence of both a static longitudinal $\mathbf{B}_{0}$ field and an oscillating transverse r.f. field $\mathbf{B}_{1}$, the spin magnetisation vector precesses about both. $\mathbf{M}$ will precess about $\mathbf{B}_{0}$ at $\omega_{0}$ and about $\mathbf{B}_{1}$ at $\omega_{1}$. This is illustrated in Fig. 3.7 (a).

We make the coordinate transformation to a reference frame rotating about the $\mathrm{z}$ axis at a frequency $\omega$ such that $\mathbf{B}_{1}$ appears stationary. The new coordinates will be denoted $x^{\prime}, y^{\prime}$ and $z$ as illustrated in Fig. 3.6 and will be used virtually exclusively throughout the rest of this and subsequent chapters. In this new rotating frame, the longitudinal field experienced by the spins is reduced by an amount $\omega / \gamma$ so that the 


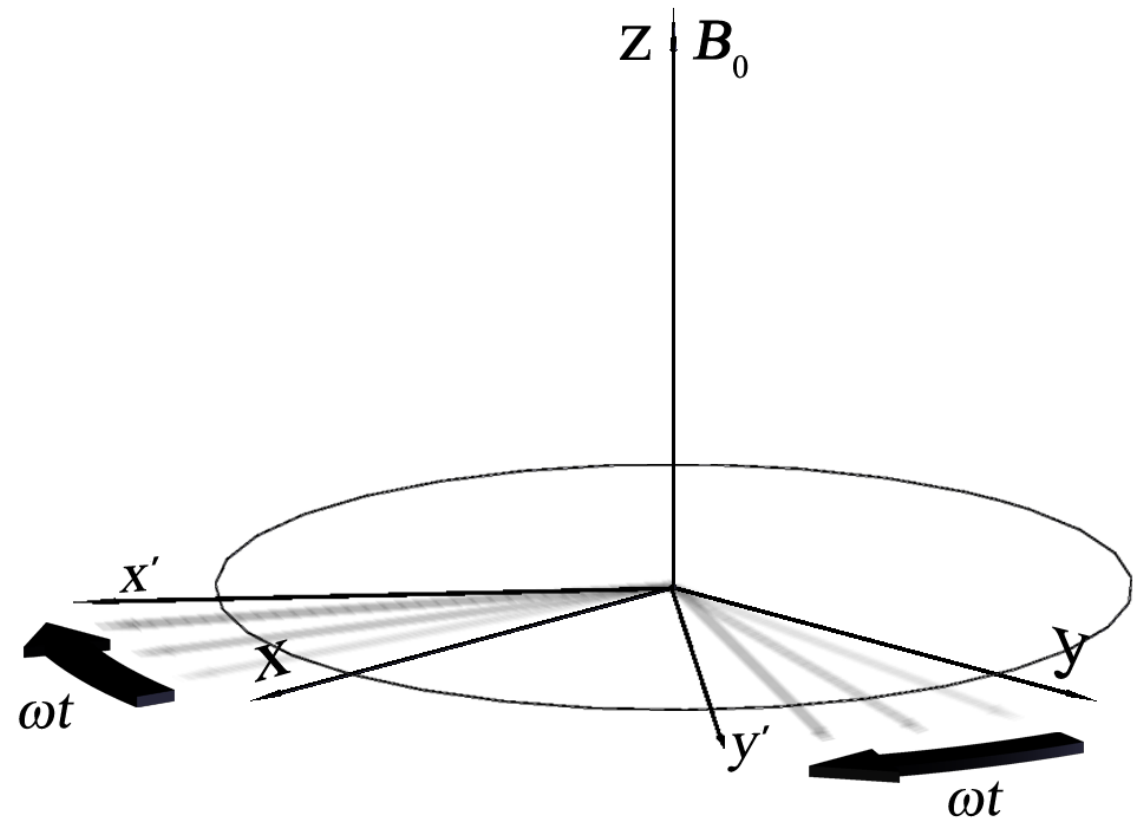

Figure 3.6: We make a transformation to a reference frame that rotates about the $\mathrm{z}$ axis at a frequency $\omega$ in the same sense as the transverse $\mathbf{B}_{1}$ field. The new coordinates are denoted $x^{\prime}, y^{\prime}$ and $z$. This reduces the complexity of the analysis and will be used virtually exclusively throughout the rest of this and subsequent chapters.

total field experienced by the spins is

$$
\begin{aligned}
\mathbf{B}_{e f f} & =\mathbf{B}^{\prime}+\mathbf{B}_{1} \\
& =\mathbf{B}_{0}-\frac{\omega}{\gamma} \mathbf{k}+\mathbf{B}_{1} \\
& =\mathbf{B}_{0}\left(1-\omega / \omega_{0}\right)+\mathbf{B}_{1} .
\end{aligned}
$$

Thus, in the absence of a transverse $\mathbf{B}_{1}$ field when the rotating frame oscillates at the NMR frequency $\omega_{0}$, the longitudinal field vanishes and the magnetisation vector appears stationary. If the frequency of the transverse r.f. field $\mathbf{B}_{1}$ matches the NMR frequency $\omega_{0}$, the only field felt by the spins is the transverse one, and the spins will precess about it at $\omega_{1}$ as shown in Fig. 3.7(b).

If the transverse field is applied at a frequency $\omega \neq \omega_{0}$ then the effective field experienced by the spins is a linear superposition of the diminished longitudinal field, 


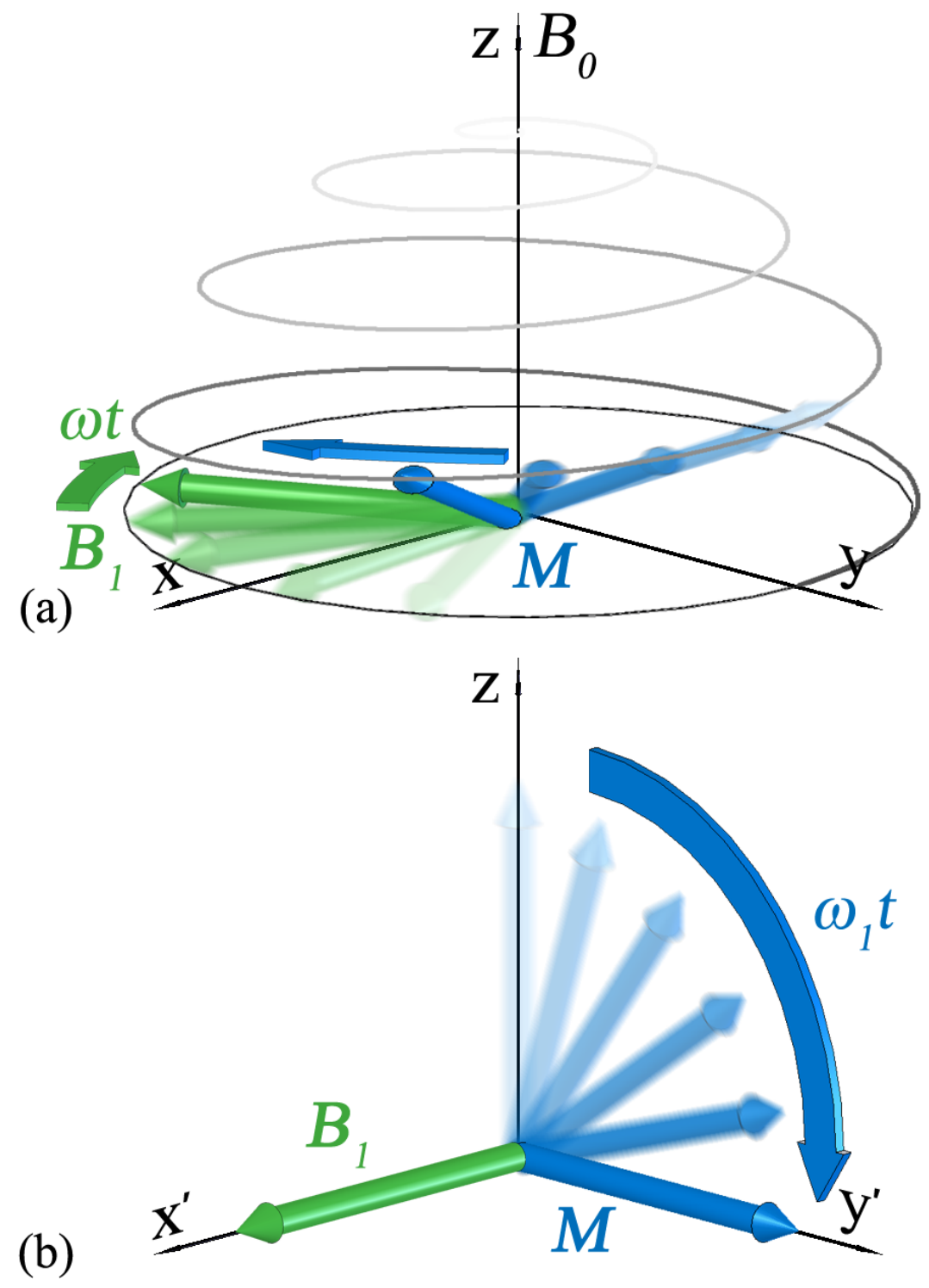

Figure 3.7: The evolution of the bulk magnetisation in the presence of a longitudinal static field $\boldsymbol{B}_{0}$ and an oscillating transverse field $\mathbf{B}_{1}$. (a) As seen in the laboratory frame. The spin magnetisation vector (shown in blue) precesses about $\mathbf{B}_{0}$, oriented along the $\mathrm{z}$ axis, at $\omega_{0}$, and the rotating $\mathbf{B}_{1}$ (shown in green) at $\omega_{1}$. (b) As seen in the rotating frame. The influence of the longitudinal $\mathbf{B}_{0}$ field disappears and the spins precess only about the transverse $\mathbf{B}_{1}$ field at $\omega_{1}$. 


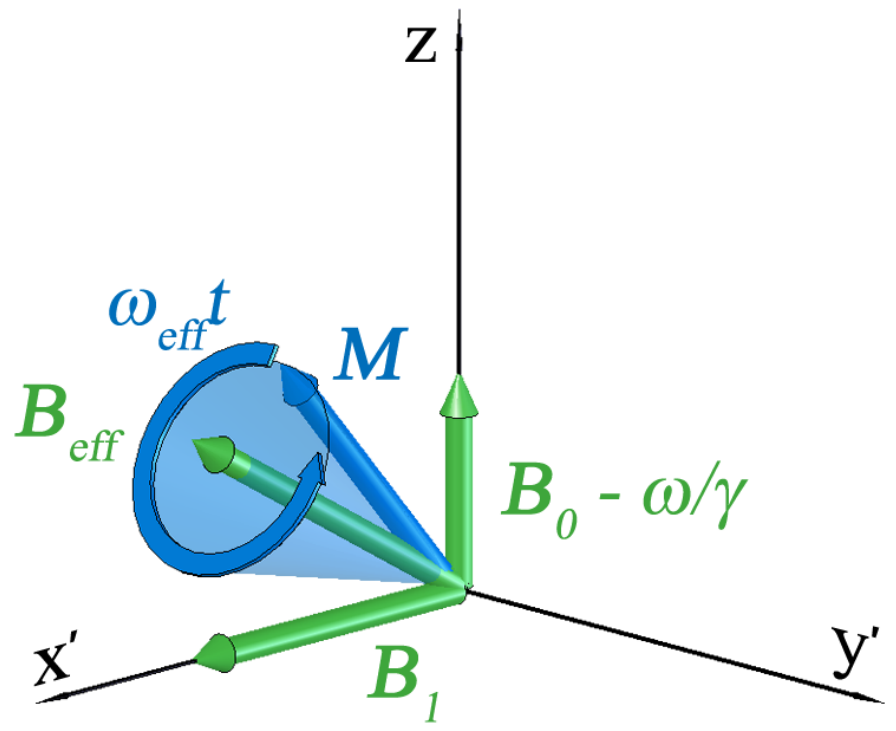

Figure 3.8: The effect of applying a transverse field, $\mathbf{B}_{1}$ off resonance as seen in the rotating frame. The effective field felt by the spins is a linear superposition of the diminished longitudinal field, $\mathbf{B}_{0}-\frac{\omega}{\gamma} \mathbf{k}$ and the transverse field $\mathbf{B}_{1}$. The spin magnetisation vector will precess about the resultant field $\mathbf{B}_{e f f}$ at a frequency $\omega_{\text {eff }} t$.

$\mathbf{B}_{0}-\frac{\omega}{\gamma} \mathbf{k}$ and the transverse field, $\mathbf{B}_{1}$ as established in equation 3.29. This situation is illustrated in Fig. 3.8. The spin magnetisation vector will therefore precess about the effective field at a frequency of $\omega_{\text {eff }}$ given by $\omega_{\text {eff }}=\gamma B_{\text {eff }}$.

The longer the transverse field is applied for, the larger that the angle the magnetisation vector is tipped through will be. If the $\mathbf{B}_{1}$ field is applied for a period of time $t_{p}$ the angle tipped through, $\theta$ will be equal to $\gamma B_{1} t_{p}$. In Fig. 3.7, the $\mathbf{B}_{1}$ field has been arbitrarily chosen to lie along the $x^{\prime}$ axis and the magnetisation vector has been tipped through $90^{\circ}$. The pulse of r.f. radiation that results in this condition is known as a $90_{x}$ pulse and leaves the magnetisation in the experimentally detectable $x^{\prime} y^{\prime}$ plane with components $M_{z}=0, M_{y}=M_{0}$. A pulse that rotates the magnetisation vector about the $x^{\prime}$ axis through $180^{\circ}$ is called a $180_{x}$ pulse. When a $180_{x}$ pulse is applied to a group of spins in thermal equilibrium with their surroundings, the populations of the energy levels are inverted and the magnetisation ends up oriented along the $-z$ axis with components $M_{z}=-M_{0}, M_{x}=M_{y}=0$. We shall use the convention $\Theta_{ \pm \mathrm{x}}$ when referring to pulses of r.f. radiation, where $\Theta=\omega_{1} t$ is the angle, in degrees, the spins are tipped through and the subscript refers to the rotating frame axis about which 
they are tipped. Combinations of various pulses of sometimes different durations with carefully selected intervals of time between them are collectively known as pulse sequences and are the NMR-scientists toolbox from which they select the appropriate tool to measure the specific behaviour they are interested in. To understand the specifics of what information can be gathered from each pulse sequence, we must understand more about the nature of pulses and how the spins relax to their equilibrium positions, the subject of the next sections.

\subsection{Pulses}

Spins can be disturbed from their equilibrium position via the application of a pulse of r.f. radiation. The frequency, duration, amplitude and shape of the pulse in the time domain determines which spins are disturbed and this relationship can be understood via the Fourier transform which is defined by the relationship

$$
\begin{aligned}
F(\omega) & =\int_{-\infty}^{\infty} f(t) \exp (-i \omega t) \mathrm{d} t \\
f(t) & =\frac{1}{2 \pi} \int_{-\infty}^{\infty} F(\omega) \exp (i \omega t) \mathrm{d} \omega .
\end{aligned}
$$

Equation 3.30 is only exactly true, however, for small tip angles. Figure 3.9(a) shows a time-domain representation of what is often called a hard pulse. A hard pulse is a burst of r.f. radiation modulated by a hat function of duration $t_{p}$. Figure 3.9(b) illustrates the frequency-domain response of the hard pulse. In the frequency domain, the hard pulse excites a sinc function defined as $\operatorname{sinc}(a \omega)=\frac{\sin (a \omega)}{a \omega}$. The bandwidth of a hard pulse of duration $t_{p}$ is of order $2 t_{p}^{-1}$. Thus, if the $B_{1}$ field is applied at a frequency $\omega$, spins precessing at frequencies within $t_{p}^{-1}$ will be excited. Typical bandwidths of hard pulses are in excess of $20 \mathrm{kHz}$, which is large enough in most cases to excite all nuclei for a given spin species.

\subsection{Relaxation}

The thermal equilibrium of an ensemble of spins in a static magnetic field is characterised by a state of polarisation with magnetisation $\mathbf{M}_{0}$ directed along the longitudinal magnetic field $\mathbf{B}_{0}$. The application of a resonant r.f. pulse of radiation disturbs 


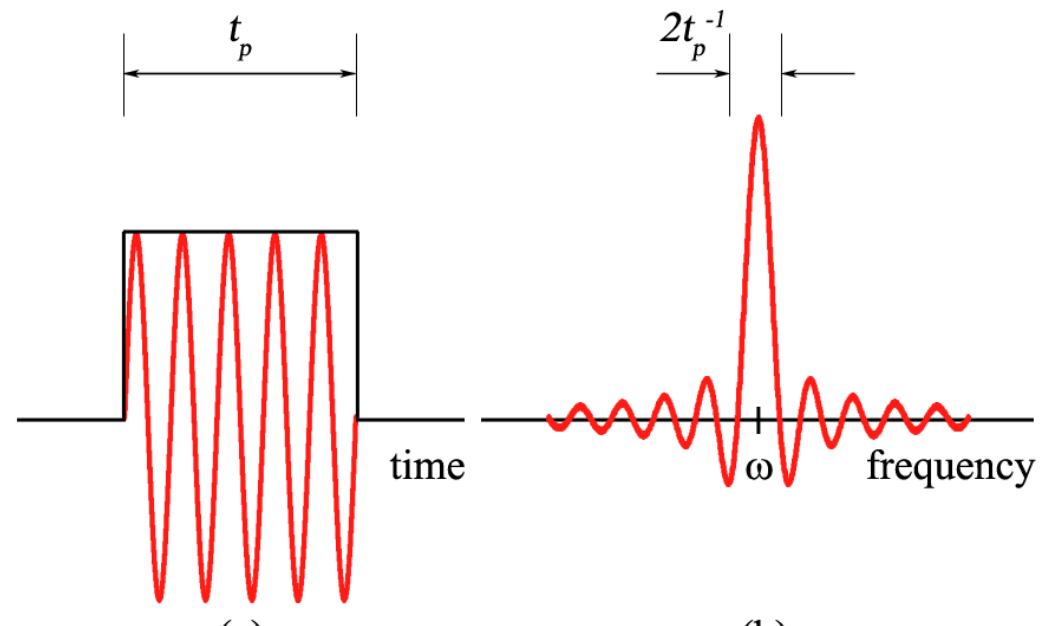

(a)

(b)

Figure 3.9: (a) A time-domain representation of a hard pulse, a burst of r.f. radiation modulated by a hat function of duration $t_{p}$. (b) The frequency spectrum associated with (a) as calculated by Fourier theory. The bandwidth of a hard pulse of duration $t_{p}$ is of order $2 t_{p}^{-1}$.

the system from this equilibrium. In time, the equilibrium will be restored via a process known as spin-lattice relaxation whereby spins exchange (thermal) energy with the surrounding thermal reservoir known as the lattice. Another relaxation mode that is of great importance is the process through which spins come to thermal equilibrium among themselves, a phenomenon known as spin-spin relaxation. These two processes contribute differently to the overall relaxation depending on the vibrational, rotational and translational motions of the spins. A study of these processes thus reveals important information about the molecular dynamics on a range of timescales. While a thorough understanding of these processes is beyond the scope of this thesis, a brief introduction will be presented. A more thorough handling is given in [11,12,14].

\subsubsection{Spin-lattice relaxation}

After the application of a resonant r.f. pulse has disturbed the system, the spins begin to return to their thermal equilibrium as illustrated in Fig. 3.10 via an energy transfer process known as spin-lattice relaxation. Fig. 3.10 shows the special case where a $180_{x, y}$ pulse has left the system in a state where $M_{z}=-M_{0}$ and the transverse magnetisation, $M_{x, y}$ is zero. There is thus only longitudinal relaxation taking place in Fig. 3.10. 


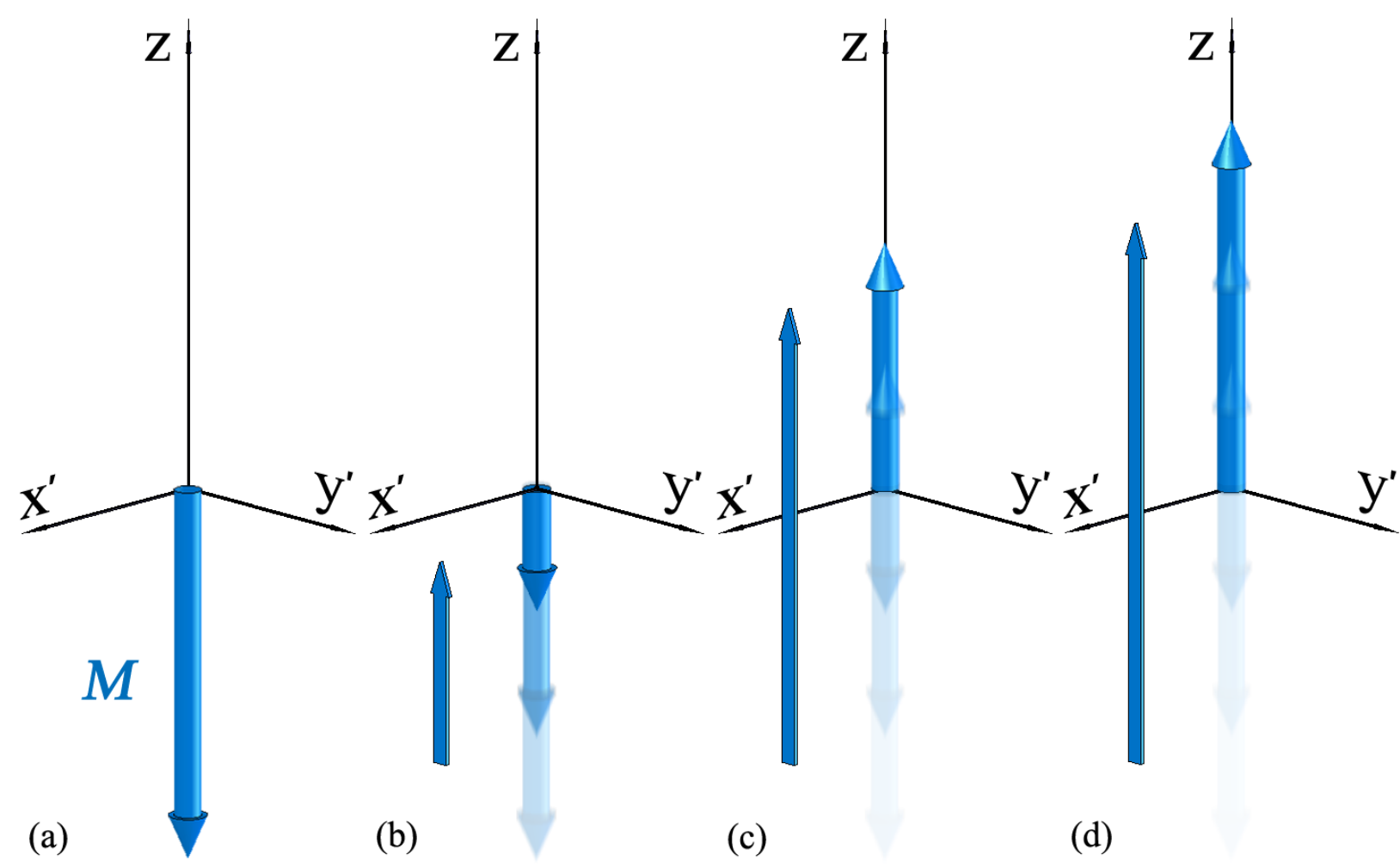

Figure 3.10: Spin-lattice (longitudinal) relaxation: The evolution of the longitudinal component of the bulk magnetisation following a $180_{x, y}$ r.f. pulse. The magnetisation can be said to recover to thermal equilibrium in an exponential manner with a time constant $T_{1}$ via exchange of (thermal) energy with the lattice.

As section 3.4 (and the name itself) suggests, spin-lattice relaxation is a process that involves the exchange of energy between the excited spins and the thermal motion of the molecules which make up the lattice. A phenomenological description of this process is given by the relation

$$
\frac{\mathrm{d} M_{z}}{\mathrm{~d} t}=-\frac{1}{T_{1}}\left(M_{z}-M_{0}\right)
$$

where $T_{1}$ is known as the spin-lattice relaxation time. Equation 3.31 has the solution

$$
M_{z}(t)=M_{z}(0) \exp \left(-t / T_{1}\right)+M_{0}\left(1-\exp \left(-t / T_{1}\right)\right)
$$

Values of $T_{1}$ typically range from 0.1 to 10 seconds at room temperature for protons in dielectric materials. 


\subsubsection{Spin-spin relaxation}

Transverse magnetisation is characterised by a state of phase coherence between spin states as discussed in section 3.5.1. The reduction of transverse magnetisation is due to the spins coming to thermal equilibrium among themselves and so is called spin-spin relaxation. This reduction in coherence results in an overall reduction of the transverse magnetisation. A phenomenological description of this process is given by the relation

$$
\frac{\mathrm{d} M_{x, y}}{\mathrm{~d} t}=\frac{-M_{x, y}}{T_{2}}
$$

where $T_{2}$ is called the spin-spin relaxation time and is the time over which the transverse magnetisation reduces by a factor of $e$. Equation 3.33 has solution

$$
M_{x, y}(t)=M_{x, y}(0) \exp \left(-t / T_{2}\right)
$$

While energy exchange with the lattice may play a role in the transverse relaxation, other terms that lead to dephasing of the spins are also responsible for the loss of transverse magnetisation. This leads to the result $T_{2} \leq T_{1}$.

Equation 3.34 describes, albeit phenomenologically, what is referred to as 'pure $T_{2}{ }^{\prime}$ relaxation. In practice, there is another major cause of transverse relaxation, that of magnetic field inhomogeneities. The magnitude of the inhomogeneity in the field is denoted $\Delta B_{0}$. Any lack of homogeneity in $\mathbf{B}_{0}$ will mean that spins will precess at different rates based on their physical position. This leads to an increase in the rate of relaxation which is described by the parameter $T_{2}^{*}$ :

$$
\frac{1}{T_{2}^{*}}=\frac{1}{T_{2}}+\frac{\gamma \Delta B_{0}}{2 \pi}
$$

It is important to note that phase coherence loss due to pure $T_{2}$ relaxation is random and irreversible, whereas the relaxation caused by inhomogeneity in $\mathbf{B}_{0}$ is ordered and can be reversed with appropriate care. 


\subsubsection{The Bloch equations}

The fusion of equations 3.24, 3.31 and 3.33 in the rotating frame shown in Fig. 3.6 results in a set of relationships known as the Bloch equations. They are named after the Swiss physicist Felix Bloch (October 23, 1905 September 10, 1983) who won the 1952 Nobel Prize for Physics for his work on nuclear magnetism.

$$
\begin{aligned}
\frac{\mathrm{d} M_{x}}{\mathrm{~d} t} & =\gamma M_{y}\left(B_{0}-\omega / \gamma\right)-\frac{M_{x}}{T_{2}} \\
\frac{\mathrm{d} M_{y}}{\mathrm{~d} t} & =\gamma M_{z} B_{1}-\gamma M_{x}\left(B_{0}-\omega / \gamma\right)-\frac{M_{y}}{T_{2}} \\
\frac{\mathrm{d} M_{z}}{\mathrm{~d} t} & =-\gamma M_{y} B_{1}-\frac{\left(M_{z}-M_{0}\right)}{T_{1}} .
\end{aligned}
$$

These provide a valuable tool for describing many nuclear magnetic phenomena.

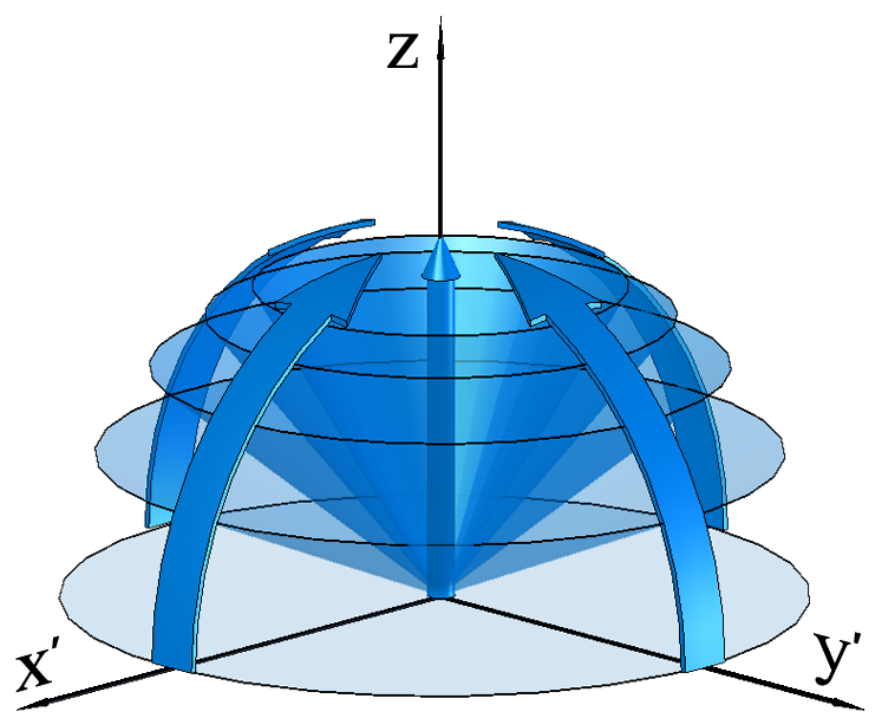

Figure 3.11: Spins that initially dephase due to $T_{2}^{*}$ relaxation will eventually relax back to thermal equilibrium via spin-lattice energy exchange. Shown here is the case where $T_{2}^{*}<<T_{1}$ and the spins dephase quickly compared with their longitudinal relaxation. 


\subsection{Signal detection and the FID}

Faraday's law of induction states that the induced electromotive force (e.m.f.) in a closed loop is directly proportional to the time rate of change of magnetic flux through the loop. Thus, in the laboratory frame following a pulse that results in a non-zero transverse magnetisation, a coil whose symmetry axis is perpendicular to the direction of the static $\mathbf{B}_{0}$ field will experience an oscillatory e.m.f. at $\omega_{0}$ due to spins precessing at the Larmor frequency. The magnitude of the e.m.f. is proportional to the amount of transverse magnetisation that exists within the coil. The primary NMR signal is measured in the time domain as a decaying oscillating signal caused by the free precession of the magnetisation. For this reason, it is known as the free induction decay and is usually referred to simply as the FID. The r.f. receivers used in the detection of NMR signals employ a method known as heterodyning. Heterodyning is the process whereby an oscillating signal is mixed with the output from a reference oscillator. The result of mixing two frequencies is two new frequencies, one which is the sum of the input frequencies and the other that is the difference between the two inputs. It is an extremely phase-sensitive method that allows the mixing of the NMR signal with two heterodyne frequencies $90^{\circ}$ out of phase with each other. This allows for detection of the orthogonal phases of the magnetisation, in effect allowing the detection of $M_{x}$ and $M_{y}$. A perfect $90^{\circ}$ r.f. pulse applied to the equilibrium state where $\mathbf{M}=M_{0} \mathbf{k}$ leaves the magnetisation entirely in the $x^{\prime}-y^{\prime}$ plane. In the laboratory frame, the magnetisation evolves as [14]

$$
\begin{aligned}
\mathbf{M}(t) & =\left[M_{0} \cos \left(\omega_{0} t\right) \mathbf{i}+M_{0} \sin \left(\omega_{0} t\right) \mathbf{j}\right] \exp \left(-t / T_{2}\right) \\
& =M_{0} \exp \left(i \omega_{0} t\right) \exp \left(-t / T_{2}\right)
\end{aligned}
$$

and the heterodyne signal at offset $\Delta \omega$ is

$$
S(t)=S_{0} \exp (i \phi) \exp (i \Delta \omega t) \exp \left(-t / T_{2}\right)
$$

where $\phi$ is the absolute receiver phase and $S_{0}$ is the signal amplitude immediately following the pulse, a value that is proportional to the equilibrium magnetisation $M_{0}$. Although the FID is a continuous signal, it is digitally sampled in $N$ discrete steps over an interval of time $T$. A Fourier transform of the time-domain signal reveals the spectrum in the frequency domain with a bandwidth equal to $1 / T$ as shown in Fig. 3.12. The spacing of the discretely sampled points is thus $1 / N T$ in the frequency domain. The 
discrete nature of the sampling is represented in Fig. 3.12 by the temporally evenly spaced black points, while the continuous nature of the process is represented by the red line. Fourier transformation of the real part of the signal results in a Lorentzian whose full-width at half-maximum (FWHM) is $2 / T_{2}$, a spectrum known as the absorption spectrum. Fourier transformation of the imaginary part of the time-domain signal is known as the dispersion spectrum and is also illustrated in Fig. 3.12.
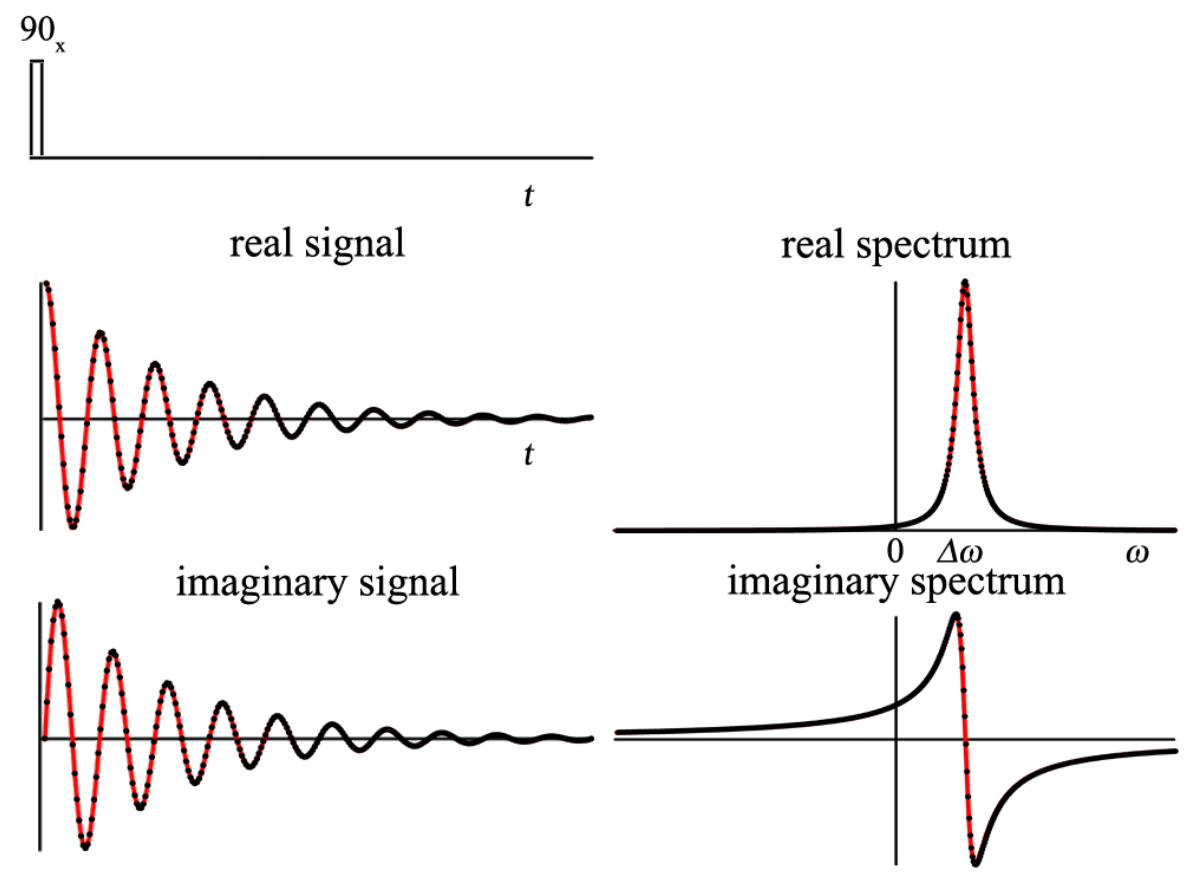

Figure 3.12: Free induction decay (FID) following a $90_{x}$ pulse. Acquisition begins immediately following the pulse where $N$ points (black) are sampled over an interval of time $T$. The red line illustrates the continuous nature of the process in contrast to the discrete nature of the sampling. Fourier transformation of the real part of the time-domain signal results in the absorption spectrum which is characterised by a Lorentzian of FWHM $2 / T_{2}$ centred at the offset frequency. Fourier transformation of the imaginary part of the time-domain signal results in what is known as the dispersion spectrum.

\subsection{Signal averaging and phase cycling}

The observation of weak effects in NMR requires high sensitivity and noise- and artefactfree signals. The best measure of these factors is the signal-to-noise $(S / N)$ ratio. The 
higher the signal-to-noise ratio, the more trustworthy peaks are and the less chance there is of erroneously assigning a peak, or missing an important yet weak feature. The two causes of a low signal-to-noise ratio are noise and artefacts. The main source of noise is Brownian motion of electrons in the receiver coil. 'White noise' is characterised by statistically-distributed frequency components that are impossible to eliminate (at a non-zero temperature), whereas artefacts are unwanted signals at well-defined frequencies that can often be eliminated by carefully designed pulse sequences. It is exceedingly difficult to distinguish artefacts from genuine signals via post-experimental data processing and so the best methods are those that reduce or eliminate artefacts completely. The simplest method for reducing the effect the noise has on the signal-tonoise ratio is to add the signal from $N$ experiments. The signal amplitude will grow in proportion to the number of acquisitions whereas, because of its random nature, the amplitude of the noise will only grow as $\sqrt{N}$. That is to say, the noise does not add coherently while the signal does. The signal-to-noise ratio will thus increase by a factor of $\sqrt{N}$ with $N$ successive experiments. For full signal to be measured each time, there must be an interval of time waited at the end of each experiment that is sufficient for full relaxation of the magnetisation to take place. This delay is usually of the order of several $T_{1}$ relaxation times. The term 'phase cycling' refers to the periodic changes made to the phase of the applied $\mathbf{B}_{1}$ field in order to reduce or eliminate artefacts caused by imperfections in the r.f. pulses and imperfections in the receiver. Perhaps the simplest phase cycle is inversion which is achieved by incrementing the r.f. pulse transmitter and receiver phases by $180^{\circ}$ in conjunction with an alternating addition and subtraction of the signal. Such a phase cycle coherently adds the desired FID signal while suppressing the unwanted signal.

\subsection{Elementary pulse sequences}

Sequences of pulses of various lengths and phases with carefully selected delays between them are known as pulse sequences and are the NMR scientists tools with which they examine molecular behaviours and structures. An exhaustive list of pulse sequences is not necessary in any NMR text, but it is a worthwhile exercise to discuss some elementary sequences. Some complex sequences are similar to simpler sequences, with extra features added in to examine specific effects with fewer distortions from artefacts. In this section we shall introduce two of the most important pulse 


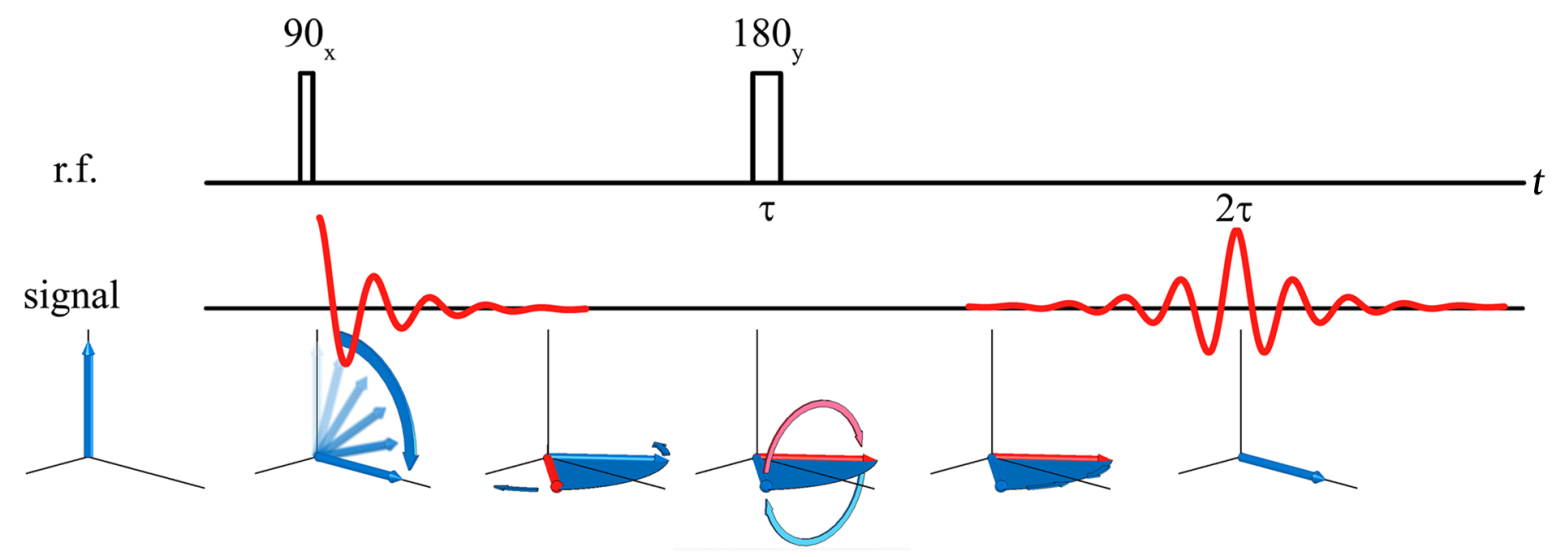

Figure 3.13: A spin echo pulse sequence. The initial $90_{x}$ pulse puts the spins into the $x^{\prime} y^{\prime}$ plane, where they begin to dephase at a rate $T_{2}^{*}$. After dephasing for a time $\tau$, a $180_{y}$ pulse reorients the spins so the spins that are precessing faster (exemplified by the red vector) are now behind those precessing slower (in blue). After a time $2 \tau$, the spins come back into phase, creating an echo. The evolution of the magnetisation vector is illustrated in the rotating frame, though it should be noted that the signal is measured in the laboratory frame.

sequences used in this thesis and also introduce two methods of measuring nuclear relaxation.

\subsubsection{Spin and stimulated echoes}

A simple two-pulse sequence was proposed by Hahn [19] in 1950 and is illustrated in Fig. 3.13. The sequence begins with a $90_{x}$ pulse that tips the spins into the $x^{\prime} y^{\prime}$ plane where they subsequently begin to dephase due to $T_{2}^{*}$ relaxation. Field inhomogeneities, a major contribution to $T_{2}^{*}$ relaxation, cause the spins to lose coherence faster than due to pure $T_{2}$ relaxation alone. A time $\tau$ after the initial pulse, a $180_{y}$ pulse is applied to the system. The $180_{y}$ pulse inverts the spins in the $x^{\prime} y^{\prime}$ plane so that spins that were precessing faster, exemplified by the red vector in Fig. 3.13, are now behind the average and those precessing slower are now ahead. This difference in precession rates causes the spins to reestablish coherence at a time $2 \tau$, causing a spin echo as indicated in Fig. 3.13.

In some materials, including those where the molecular motion is slow compared with the Larmor precession, the transverse relaxation can be much more rapid than 
the longitudinal relaxation. When such a scenario exists, it is often preferable to 'store' the magnetisation out of the $x^{\prime} y^{\prime}$ plane so that the spins undergo $T_{1}$ relaxation only. A pulse sequence that performs this is shown in Fig. 3.14. The initial $90_{x}$ pulse acts in the same way as for the spin echo case shown in Fig. 3.13. After a time $\tau$, another $90_{x}$ pulse is applied which acts to tip the spins into the $x^{\prime} z$ plane. There is no effect on the spins that are oriented along the $x^{\prime}$ axis and so only half the magnetisation is stored. The spins are 'stored' along the $z$ axis for a time $T$ subject only to $T_{1}$ relaxation before being tipped into the $x^{\prime} y^{\prime}$ axis again by the third $90_{x}$ pulse. Coherence is reestablished in the transverse plane a time $\tau$ after the third pulse, giving rise to an echo. Because the spins are stimulated back into the transverse plane after being stored for a time $T<T_{1}$ along the $z$ axis, this type of sequence is called a stimulated echo. It should be noted that there are two spin echoes formed in this sequence, and special care must be taken in order to separate the stimulated echo from the spin echoes. A particularly effective technique for doing this is to incorporate a pulse of magnetic field gradient in the $x^{\prime} y^{\prime}$ plane while the magnetisation is stored along the $z$ axis. This pulse destroys any F.I.D caused by the second $90_{x}$ pulse and is referred to as a homospoil and is shown in green in Fig. 3.14.

\subsubsection{Measurements of nuclear relaxation}

In principle, the spin echo method of the previous section is capable of eliminating the relaxation caused by field inhomogeneities so the amplitude of the spin echo is governed by $T_{2}$ relaxation alone. Carr and Purcell suggested [20] an extension to the spin echo of Hahn that consisted of repeating $180_{x}$ pulses after the initial $90_{x}$ pulse. In such a sequence, echoes are formed at times $2 \tau, 4 \tau,(2 n) \tau$ with alternating sign. By plotting the maximum amplitude of the echo as a function of time, the relaxation curve can be obtained. This method relies on perfect pulses, which in practice do not occur. The result of accumulated phase errors in the $180_{x}$ pulses is a relaxation curve that relaxes faster than the pure $T_{2}$ relaxation curve. The problem of accumulated phase errors was overcome by Meiboom and Gill [21] when they suggested the $180^{\circ}$ pulses have a different phase from the initial $90^{\prime}$ pulse. Orienting the inversion pulses along the $y^{\prime}$ axis means that any phase errors from one pulse are cancelled by the following pulse and all echoes have the same sign. This sequence, known as the Carr-PurcellMeiboom-Gill (CPMG) echo train, is illustrated in Fig. 3.15. The envelope of the echoes 


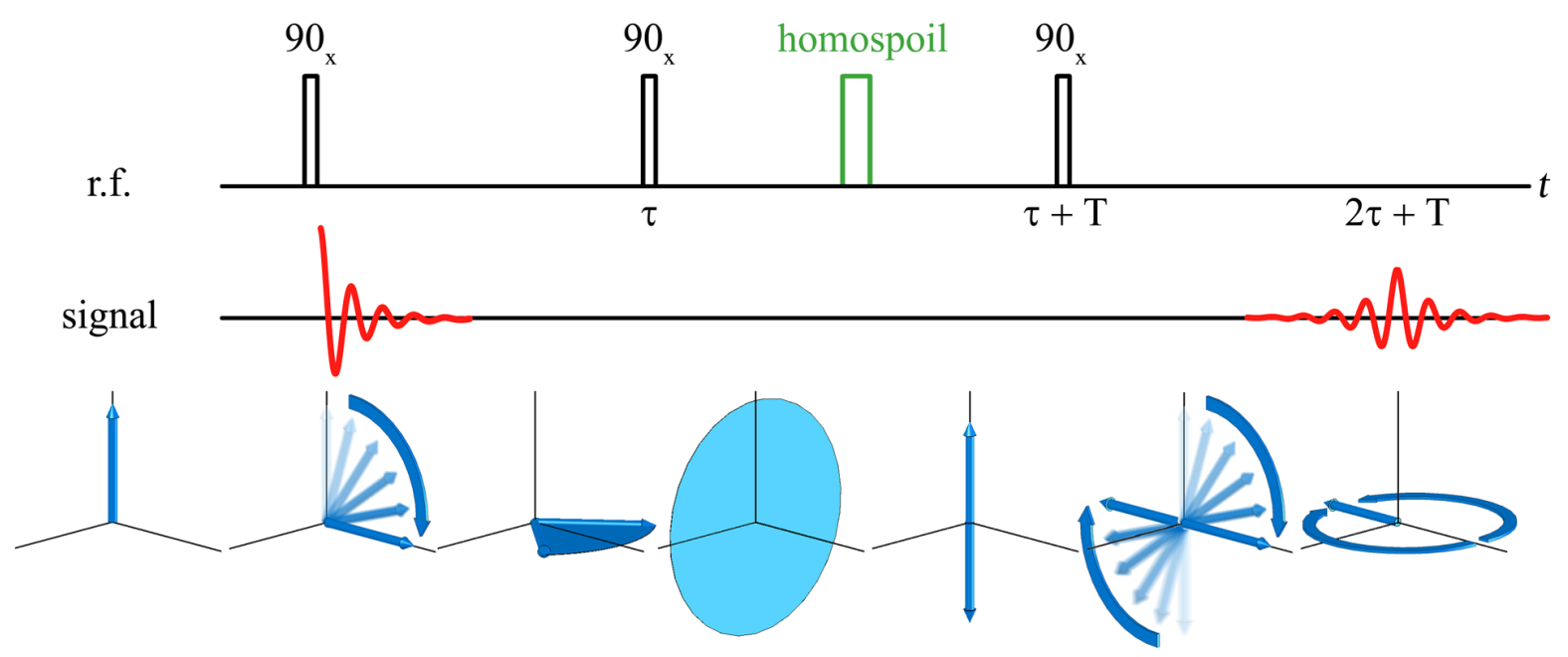

Figure 3.14: A stimulated echo pulse sequence. The initial $90^{\circ}$ pulse tips the spins into the transverse plane where they begin to dephase. The second $90^{\circ}$ pulse tips the (dephased) spins into the $x^{\prime} z$ plane after a time $\tau$, where the longitudinal component is subject to $T_{1}$ relaxation while the transverse component continues to dephase and is lost. The magnetisation is 'stored' along the $z$ axis for a time $T$ before being brought back into the transverse plane. During this time, a pulse of magnetic gradient called a homospoil, applied in the $x^{\prime} y^{\prime}$ plane, destroys the F.I.D. from the second $90_{x}$ pulse. A time $\tau$ after the spins are brought back into the $x^{\prime} y^{\prime}$ plane, partial coherence is established and an echo is formed.

in a CPMG experiment can be used to determine the spin-spin relaxation constant $T_{2}$ in a single experiment.

Measurement of the spin-Lattice relaxation constant, $T_{1}$ is easiest performed via the inversion recovery method illustrated in Fig. 3.16. The magnetisation is inverted by a $180_{x}$ r.f. pulse and then allowed to relax vis spin-lattice energy exchange. A time $T$ after initial inversion, the spins are tipped into the transverse plane and the FID is measured. The magnetisation measured in the transverse plane immediately following the $90_{x}$ pulse is equal to the longitudinal magnetisation prior to the pulse. The signal amplitude is described by

$$
M(T)=M_{0}\left[1-2 \exp \left(-T / T_{1}\right)\right]
$$

so that a series of experiments where the time between inversion and inspection, $T$, is varied will yield the spin-lattice relaxation curve. It is worth noting that a null signal is measured when $T=\ln 2 T_{1}$ so that $T_{1}$ can be measured in a single experiment. The fact that the magnetisation has a definite null can also be used to suppress the signal 


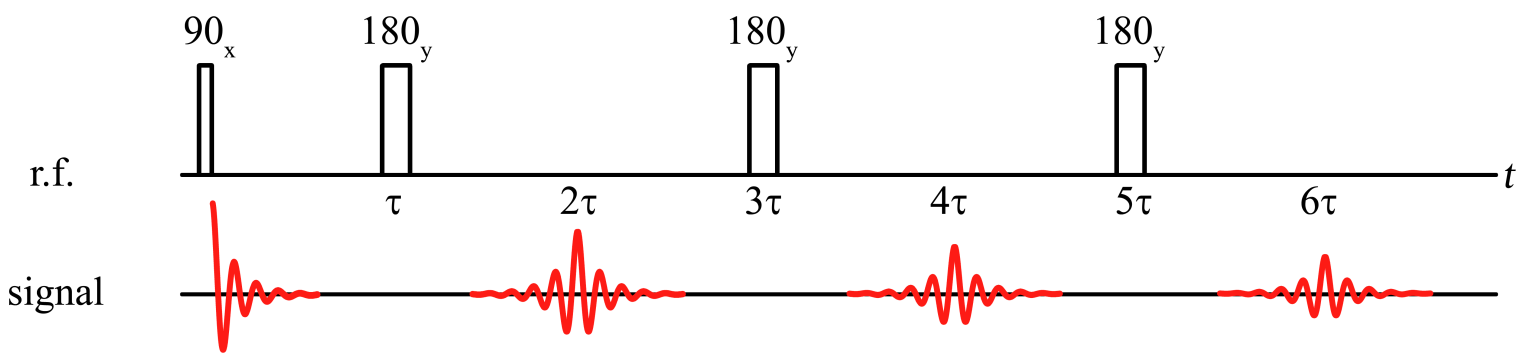

Figure 3.15: The Carr-Purcell-Meiboom-Gill pulse train. A series of spin echoes are formed at times $2 n \tau$. The envelope of the echoes is governed by $T_{2}$ relaxation only.

from a spin with a specific $T_{1}$ value by applying an inversion pulse $\ln 2 T_{1}$ before the sequence.

\subsection{Imaging}

In NMR spectroscopy, it is preferable to have highly homogeneous magnetic fields inside the sample to ensure the broadening due to inhomogeneities is small compared to the natural linewidth of spectral features. In contrast, known inhomogeneities, in the form of constant magnetic field gradients, can be imposed to encode for spatial position. This principle lies behind magnetic resonance imaging (MRI).

\subsubsection{Spin density and k-space}

When a linear magnetic field gradient, G, is applied to a collection of spins in the presence of a polarising field $\mathbf{B}_{0}$, the local Larmor frequency is altered so that we may write

$$
\omega(\mathrm{r})=\gamma B_{0}+\gamma \mathrm{G} \cdot \mathrm{r} .
$$

where $\mathbf{r}$ is the vector representing the nuclear spin coordinate. The field gradient $\mathbf{G}$ is defined as the grad of the pulsed field component parallel to $\mathbf{B}_{0}$. Consider the collection of spins at a point $\mathbf{r}$ occupying a volume element $\mathrm{d} V$. There will thus be $\rho(\mathbf{r}) \mathrm{d} V$ spins in this volume if the density can be written as $\rho(\mathbf{r})$. Equation 3.37 can then be employed to express the signal obtained from the volume as

$$
\mathrm{d} S(\mathbf{G}, t) \propto \rho(\mathbf{r}) \mathrm{d} V \exp [i \omega(\mathbf{r}) t]=\rho(\mathbf{r}) \mathrm{d} V \exp \left[i\left(\gamma B_{0}+\gamma \mathbf{G} \cdot \mathbf{r}\right) t\right]
$$



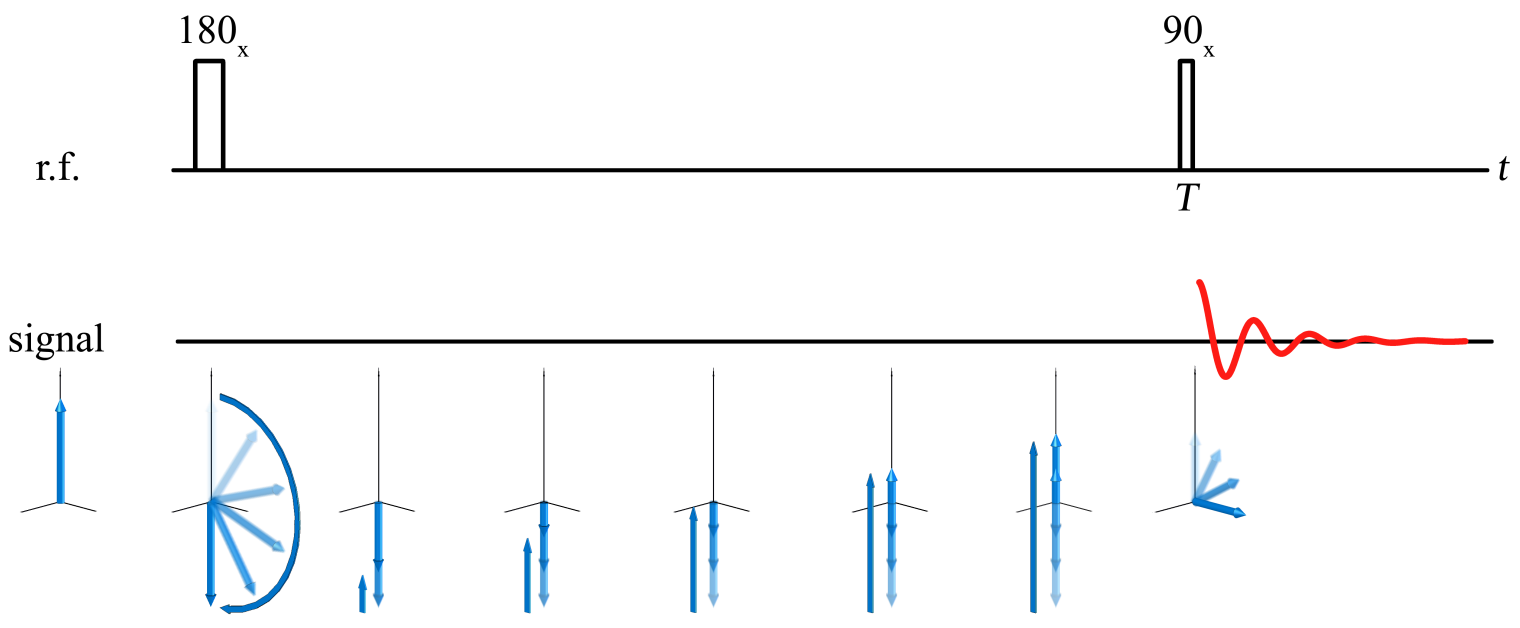

Figure 3.16: The inversion recovery method for measuring spin-lattice relaxation. The magnetisation is initially inverted and then allowed to relax via spin-lattice energy exchange for a time $T$ when it is tipped into the experimentally measurable transverse plane. The magnitude of the magnetisation immediately following the $90_{x}$ pulse is equal to the longitudinal magnetisation immediately prior to the pulse.

where, for simplicity, we have dropped the constant of proportionality and reduced the relation to an equality. Equation 3.41 assumes the spin dephasing caused by the application of the gradient is much more rapid than that due to spin-spin relaxation. Under these circumstances, the coherence loss due to transverse relaxation can be ignored. The contribution of the $\gamma B_{0}$ term in equation 3.41 can be eliminated via heterodyne mixing using a reference frequency of $\gamma B_{0}$ so that the final signal oscillates at $\gamma \mathbf{G} \cdot \mathbf{r}$. The integrated signal amplitude may then be expressed as

$$
S(t)=\iiint \rho(\mathbf{r}) \exp [\mathrm{i} \gamma \mathbf{G} \cdot \mathbf{r} t] \mathrm{d} \mathbf{r}
$$

where $\mathrm{d} \mathbf{r}$ is a volume element and it is assumed the integration is over all space. We now introduce the reciprocal space vector $\mathbf{k}$ defined by

$$
\mathbf{k}=\frac{\gamma \mathbf{G} t}{2 \pi} \text {. }
$$

$\mathbf{k}$ has the units of inverse space, $m^{-1}$. Movement through $\mathbf{k}$-space can be achieved by altering the magnitude of the gradient, or by moving through time. The direction of travel through $\mathbf{k - s p a c e}$ is dictated by the sign of the gradient. We can now express the 
total signal of equation 3.42 using the formalism of $\mathbf{k}$-space as

$$
S(\mathbf{k})=\iiint \rho(\mathbf{r}) \exp [i 2 \pi \mathbf{k} \cdot \mathbf{r}] \mathrm{d} \mathbf{r} .
$$

The Fourier inverse of the signal is

$$
\rho(\mathbf{r})=\iiint S(\mathbf{k}) \exp [-\mathrm{i} 2 \pi \mathbf{k} \cdot \mathbf{r}] \mathrm{d} \mathbf{k} .
$$

Equations 3.44 and 3.45 state that the signal, $S(\mathbf{k})$, and the spin density, $\rho(\mathbf{r})$ are mutually conjugate. This is the fundamental relationship of NMR imaging.

\subsubsection{Selective pulses}

In NMR imaging, it is important to have the ability to selectively excite part of the sample only. This is carried out through the use of 'soft', spatially-selective pulses. Recalling section 3.6, we note that the frequency response of a pulse is found from its Fourier transform. To excite a narrow, sharply-defined band of spins in a sample, a sinc-modulated pulse is employed in conjunction with a magnetic field gradient. As illustrated in Fig. 3.17 and alluded to by Fig. 3.9, the frequency response of an r.f. pulse modulated by a sinc function is a rectangular hat function. Consequently, only spins precessing at frequencies covered by the hat function are excited and these spins will lie in a band perpendicular to the magnetic field gradient. In practice, the sinc function is truncated to a number of lobes which distorts the frequency response. In Fig. 3.17 the sinc function has been truncated to one lobe which causes distortions in the form of small side lobes in the frequency domain. This has the effect of exciting spins outside of the selected frequency range. When a $90^{\circ}$ soft pulse of duration $T$ is applied to a system, the spins will dephase in the transverse plane due to the applied field gradient. To undo this dephasing, a negative gradient (that is, a gradient of opposite sign from that applied simultaneously to the soft pulse) of duration $T / 2$ is required. Such a rephasing gradient pulse is not required when using a $180^{\circ}$ pulse due to symmetry arguments. To excite a band of spins of physical width $\Delta z$ with a soft pulse with bandwidth of $\Delta \omega$ a gradient of magnitude $G_{z}=\frac{\Delta \omega}{\gamma \Delta z}$ needs to be applied.

\subsubsection{Motion and q-space}

Motion due to flow and diffusion can be measured through the use of pulsed gradients. A pulsed-gradient-spin-echo (PGSE) sequence is shown in Fig. 3.18(a) and consists of 

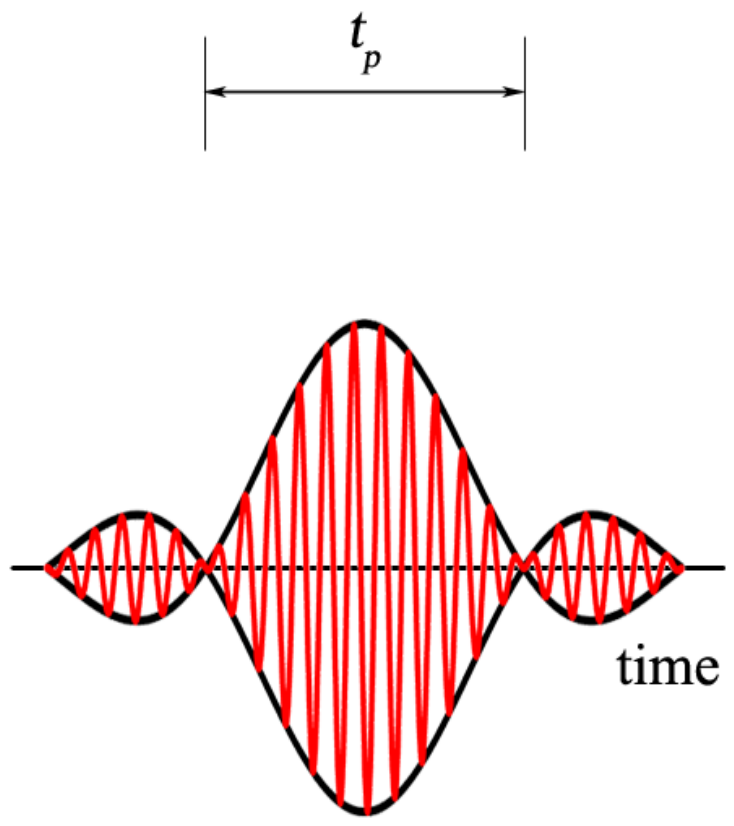

(a)
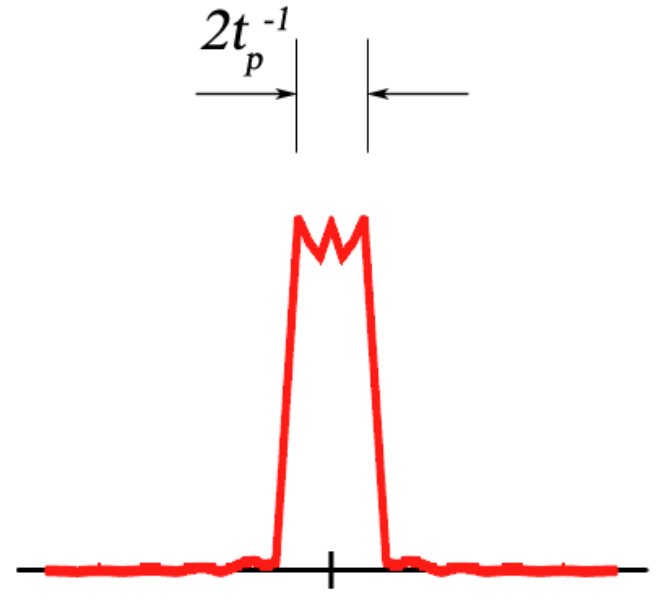

$\omega$

frequency

(b)

Figure 3.17: A 'soft' sinc-modulated r.f. pulse used for slice slection (a) in the time domain and (b) the frequency domain. The sinc modulation is truncated to one lobe which causes oscillations in the ideally-rectangular spectral response. This leads to a number of spins outside of the selected area being excited.

two gradients of duration $\delta$, a time $\Delta$ apart, either side of a $180_{y}$ pulse in a spin echo sequence. Figure 3.18(b) shows a pulsed-gradient-stimulated-echo (PGSTE), in which the gradient pulses are applied just before the spins are tilted into the $x^{\prime} z$ plane and just after their return to the transverse plane. In both cases, after the initial $90_{x}$ pulse, the first gradient imposes a phase shift equal to $\gamma \delta \mathbf{g} \cdot \mathbf{r}$ on a spin located at $\mathbf{r}$. The $180_{y}$ pulse in the PGSE and the combination of the two $90_{x}$ pulses in the PGSTE invert the original phase shift so that a molecule that has moved to a position $\mathbf{r}^{\prime}$ during the observation time $\Delta$ experiences a total phase shift of $\gamma \delta \mathbf{g} \cdot\left(\mathbf{r}^{\prime}-\mathbf{r}\right)$. If we express the spatial probability a molecule will move from $\mathbf{r}$ to $\mathbf{r}^{\prime}$ in a time $\Delta$ as $P_{s}\left(\mathbf{r} \mid \mathbf{r}^{\prime}, \Delta\right)$ then the total echo signal, $E(\mathbf{g}, \delta, \Delta)$, which we define as the amplitude of the echo at its centre, has the form

$$
E(\mathbf{g}, \delta, \Delta)=\int \rho(\mathbf{r}) \int P_{s}\left(\mathbf{r} \mid \mathbf{r}^{\prime}, \Delta\right) \exp \left[\mathbf{i} \gamma \delta \mathbf{g} \cdot\left(\mathbf{r}^{\prime}-\mathbf{r}\right)\right] \mathrm{d} \mathbf{r}^{\prime} \mathrm{d} \mathbf{r}
$$


It is now convenient to introduce the dynamic displacement $\mathbf{R}$, which is defined as $\mathbf{R}=\mathbf{r}^{\prime}-\mathbf{r}$, and the average propagator $\overline{P_{s}}(\mathbf{R}, \Delta)$, which gives the average probability that any particle will have a dynamic displacement $\mathbf{R}$ during the observation time $\Delta$. The average propagator is defined [22] by the relation

$$
\overline{P_{s}}(\mathbf{R}, \Delta)=\int P_{s}(\mathbf{r} \mid \mathbf{r}+\mathbf{R}, \Delta) \rho(\mathbf{r}) \mathrm{d} \mathbf{r} .
$$

We can now express the total echo signal of equation 3.46 in terms of the dynamic displacement and average propagator as

$$
E(\mathbf{g}, \delta, \Delta)=\int \overline{P_{s}}(\mathbf{R}, \Delta) \exp [\mathrm{i} \gamma \delta \mathbf{g} \cdot \mathbf{R}] \mathrm{d} \mathbf{R} .
$$

The signal attenuation has been removed by normalisation using the amplitude of the signal at zero gradient. It is clear from equation 3.48 that $E(\mathrm{~g}, \delta, \Delta)$ and $\overline{P_{s}}(\mathbf{R}, \Delta)$ are Fourier conjugates. Analogous to the k-space definition of section 3.11.1, we define another reciprocal space $q$ by

$$
\mathbf{q}=\frac{\gamma \delta \mathbf{g}}{2 \pi}
$$

Equation 3.48 can be expressed in terms of the formalism of $\mathbf{q}$-space as

$$
E(\mathbf{q}, \Delta)=\int \overline{P_{s}}(\mathbf{R}, \Delta) \exp [\mathrm{i} 2 \pi \mathbf{q} \cdot \mathbf{R}] \mathrm{d} \mathbf{R} .
$$

Acquisition of the signal in q-space will thus allow the imaging of the average propagator, $\overline{P_{s}}(\mathbf{R}, \Delta)$, in the same way that acquiring signal in $\mathbf{k}$-space allows for imaging of the spin density. As suggested in section 3.10.1, materials that have spin-spin relaxations approximately equal to their spin-lattice relaxation time are well suited for the PGSE shown in Fig. 3.18(a) and materials that have spin-spin relaxation that is much more rapid than their spin lattice relaxation are better suited to the PGSTE illustrated in Fig. 3.18(b).

\subsubsection{Translational dynamics - self diffusion and flow}

Random translational motion of individual spins due to thermal fluctuations, known as self-diffusion, is described statistically by the rate of change of the probability a particle will change positions by $\mathbf{R}$ in a time $t$. This is sometimes known as Fick's second law,

$$
\frac{\partial P_{s}}{\partial t}=D \nabla^{2} P_{s}
$$


where $D$ is the molecular self-diffusion coefficient. Setting the boundary condition for unrestricted self-diffusion (the probability of a particle diffusing a long distance is less than a particle diffusing a short distance) and using the initial condition $P_{s}(\mathbf{r} \mid \mathbf{r}+\mathbf{R}, 0)=$ $\delta(\mathbf{R})$ (the chance of finding a particle at its starting point is 1 ), the solution is

$$
P_{s}(\mathbf{R}, t)=(4 \pi D t)^{-3 / 2} \exp \left[-\mathbf{R}^{2} / 4 D t\right] .
$$

That $P_{s}(\mathbf{R}, t)$ relies only on the displacement $\mathbf{R}$ and not the initial location, (r), reflects the Markov nature (that is to say that given the current state, the future states are independent of the past states) of Brownian motion statistics. When measuring diffusion in one direction only, the z-direction for instance, we can integrate over the remaining two directions and the result is then

$$
P_{s}(Z, t)=(4 \pi D t)^{-1 / 2} \exp \left[-Z^{2} / 4 D t\right]
$$

with signal attenuation from a PGSE experiment given by

$$
E(q, t)=\exp \left[-4 \pi^{2} q^{2} D \Delta\right]
$$

Equation 3.54 was achieved by assuming no motion during the time, $\delta$ the gradient pulses are applied. In reality, there is some motion during this time and a correction of $\Delta-\delta / 3$ must replace $\Delta$ [14]. For the case where a coherent flow of velocity $\mathbf{v}$ is imposed on the system, equation 3.51 must have a term $\nabla \cdot \mathbf{v} P_{s}$ ammended to the right side:

$$
\frac{\partial P_{s}}{\partial t}=\nabla \cdot \mathbf{v} P_{s}+D \nabla^{2} P_{s}
$$

If $\mathbf{v}$ is constant, then equation 3.55 has solution

$$
P_{s}(\mathbf{R}, t)=(4 \pi D t)^{-3 / 2} \exp \left[-\mathbf{R}^{2} / 4 D t\right]
$$

If we again consider the case where the motion is measured in one direction only, and taking this direction to lie along the $\mathrm{z}$-axis, we can express the average propagator and echo signal attenuation as

$$
\begin{aligned}
P_{s}(Z, t) & =(4 \pi D t)^{-1 / 2} \exp \left[-\left(Z-v_{z} t\right)^{2} / 4 D t\right] \\
E(q, t) & =\exp \left[-4 \pi^{2} q^{2} D(\Delta-\delta / 3)+\mathrm{i} 2 \pi q v_{z} \Delta\right] .
\end{aligned}
$$

The position of the peak of the average propagator is thus a function of the component of velocity examined, $v$, while the width of the propagator is a function of the diffusion coefficient, $D$ as shown in Fig. 3.19. It is therefore possible to create a velocity map of a fluid under flow by measuring the average propagator for the system for a discrete number of spatial points and plotting the position of the peak. 

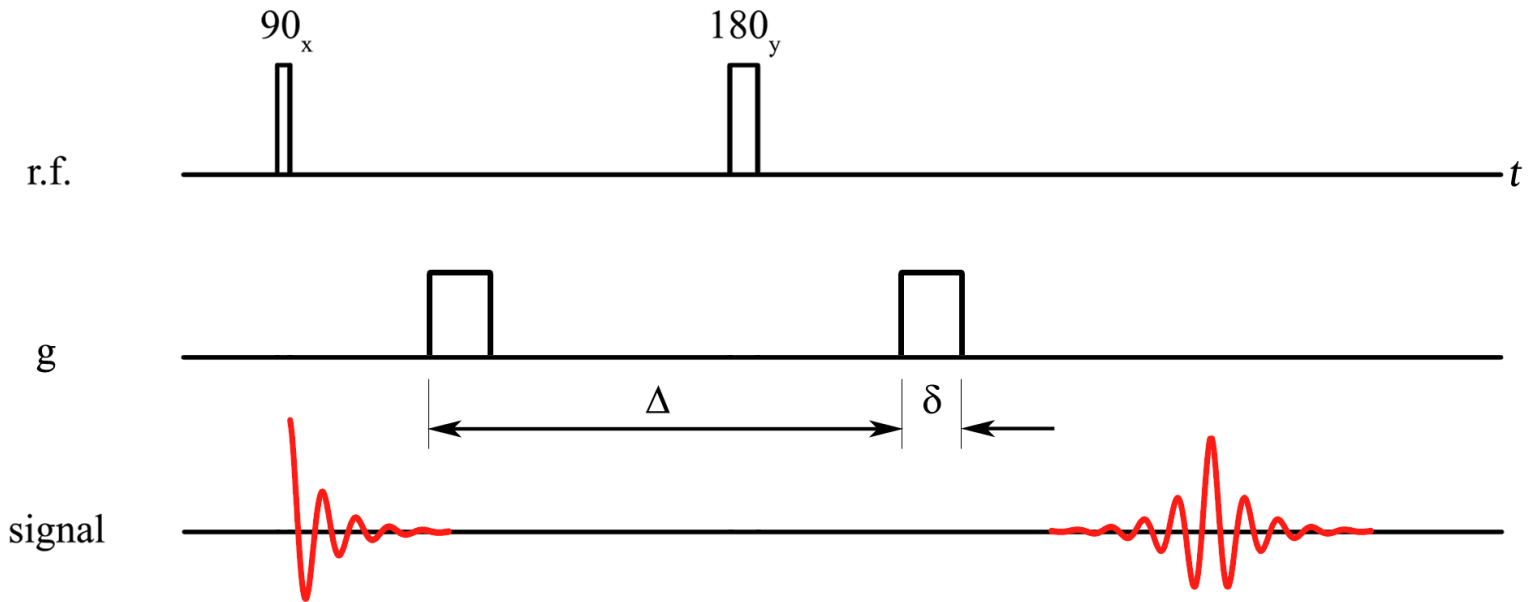

(a)
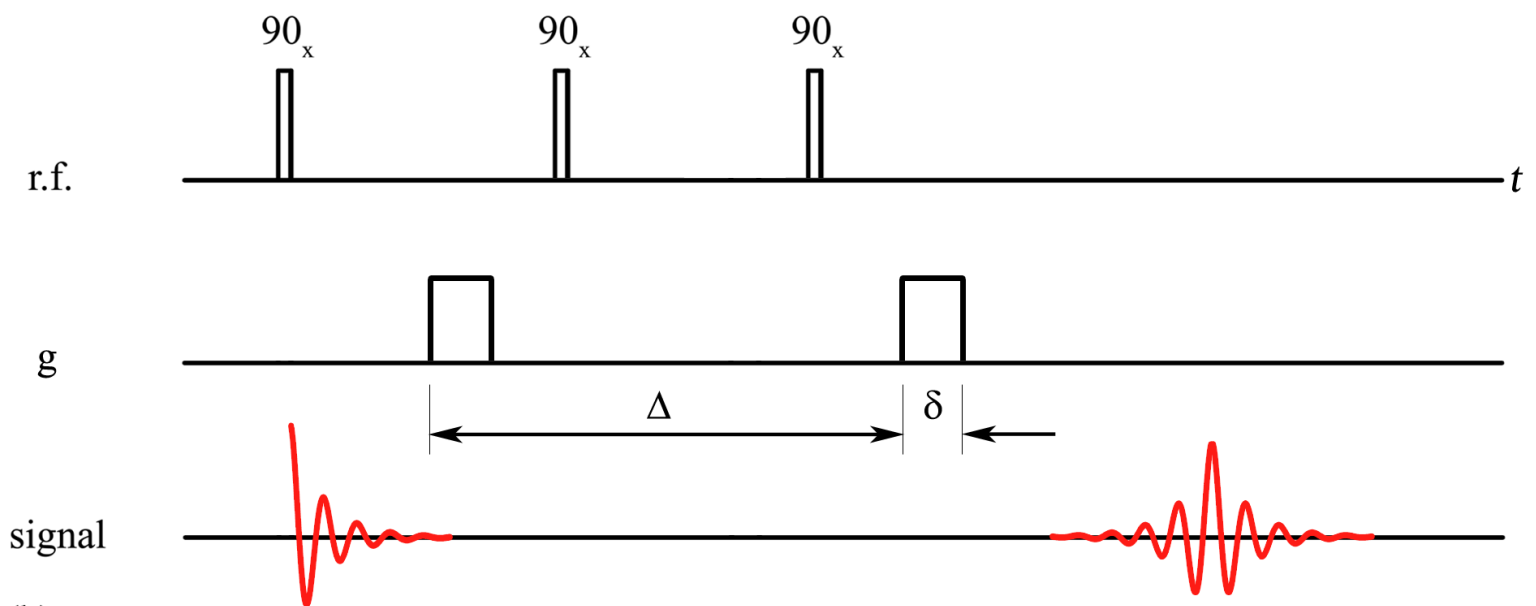

(b)

Figure 3.18: (a)Pulsed gradient spin echo (PGSE) sequence and (b) pulsed gradient stimulated echo (PGSTE) for measuring motion. PGSE is generally used for materials where $T_{2} \approx T_{1}$, and PGSTE when $T_{2}<<T_{1}$. 


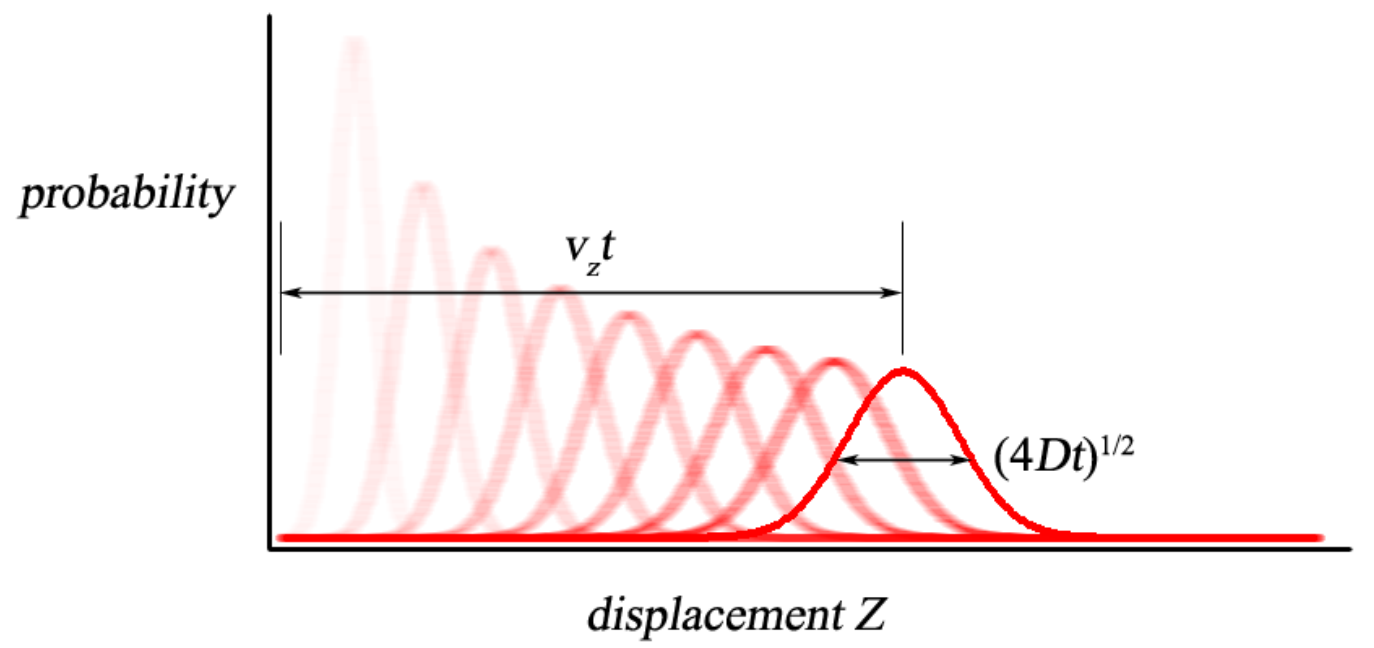

Figure 3.19: Behaviour of the average propagator, $P_{s}$ for an ensemble of particles undergoing diffusive motion and flow. The position of the peak is proportional to the bulk flow velocity, $v$, while the width is proportional to the diffusion coefficient, $D$. 


\section{Chapter 4}

\section{Soft Matter}

\subsection{Introduction}

If the experimental and theoretical work contained in this thesis can be grouped under a single name that is inherently interdisciplinary, that name is soft condensed matter physics. Soft condensed matter physics addresses fundamental research problems and has widespread technological applications. Experimental work in this field has shown that materials with different three-dimensional geometries often display similar behaviours, implying a potential universality. One example of a shared characteristic observed in complex fluids under conditions of high volume fraction is the phenomenon of "jamming" [23]. Jamming is also known as kinetic frustration, and one important class of jammed/kinetically frustrated systems is that of the glasses.

\subsection{Experimental Systems}

Much of the initial work in the field of soft glasses was carried out on densely packed hard sphere or linear polymeric chain systems. Experiments have suggested that these two topologically different classes of soft glass share many physical properties. They both, for example, share solid-like properties and show a strong dependence of self diffusion coefficient on volume fraction. It is possible to think of these two classes of soft glass as sitting at either end of a 'shape spectrum'. The differences in shape between these two classes, as well as the scale differences makes the study of the intervening parts of the spectrum necessary. The intervening parts are made of star polymers with 
varying functionalities. Highly functional stars, that is, stars with many arms, are geometrically similar to hard spheres while low functionality stars are closely related to linear chains [24, 25, 26, 27].

It has been shown that star pair interactions depend strongly on functionality, $f$ [26]. Suspensions of stars form glasses similar to hard spheres and other soft colloids [28, 29]. Multi-arm star polymers with functionality, $f$, higher than about 80 may be found in frustrated glassy states when their effective volume fraction is increased above a critical value. Systems of stars with nominal functionalities $f=128$ and various molecular weight arms are considered to be soft (they can be thought of as ultrasoft colloidal spheres with a very small deformable core and a corona consisting of arms) and have been shown to have intriguing thermal and flow properties [30,31]. It has been shown by Kapnistos et al. [30] that an increase in temperature causes suspensions of these stars at $10-15$ times the overlap concentration, $c^{*}$, to stiffen due to thermal swelling of the stars. Holmes et al. [31] showed a marked hysteresis in the response to flow of a dense solution of stars with nominal functionalities $f=128$ when probed with an upward and downward rate sweep.

The anionic synthesis of nearly monodisperse multiarm star 1,4-polybutadienes based on chlorosilane chemistry with nominal functionality, $f$, of 128 arms, each having nominal molecular mass, $M_{a}$, of $80000 \mathrm{~g} / \mathrm{mol}$, has been described in detail elsewhere [25]. All samples were prepared in squalene (molecular weight $410.73 \mathrm{~g} / \mathrm{mol}$, $T_{b}=558 \mathrm{~K} @ 25 \mathrm{~mm} \mathrm{Hg}$ ). The raw material was left in vacuum at room temperature for a period of 3 hours to remove all moisture before preparation. In squalene at $293 \mathrm{~K}$ the 128-arm stars have a $R_{H}=53 \mathrm{~nm}$, measured by light scattering techniques. Antioxidant (4-methyl-2, 6-ditert-butylphenol) was added to ensure no oxidation of the sample took place.

\subsection{Behaviour of dense colloids}

When considering how dense systems behave, the primary concern is that of the topological constraints placed upon each constituent by its nearest neighbours. In the case of densely packed linear polymeric chains at high volume fraction, $\phi$, the large number of neighbours causes entanglements which confine a given chain to a tube $[32,33,34]$ as suggested by the illustration of Fig. 4.1 (a). The dynamics of this system evolve by constituents diffusing out of their tubes, a process named 'reptation' [35]. When 
hard spheres reach a volume fraction $\phi \sim 0.58$, they form a glass [36]. In this situation, the movement of each colloid is hindered by its neighbours. That is, the neighbours form an effective cage $[37,38,39]$ which must be broken for macroscopic flow to take place as illustrated in Fig. 4.1 (b). Motions within cages are not hindered and occur on (fast) timescales. This process is referred to as $\beta$-relaxation, whereas cage breaking is a slower process and is known as $\alpha$-relaxation. In the intermediate case of colloidal stars, macroscopic flow is induced in a similar way to hard spheres; the cage formed by the nearest neighbours must be broken. However, the dynamics are not completely identical and the cage model must be modified to describe the interactions possible with stars. Because of the conformation of the stars, interpenetration of arms must also be overcome in order to break a cage. The process that describes this retraction of arms is similar to the idea of reptation with a tethered end. The case of overlapping star polymers is illustrated in Fig. 4.1 (c).

Pearson and Helfand [40] considered the problem of stress relaxation for a single star polymer in a fixed network of entanglements after a small affine step strain. As stated above, a single star cannot reptate to recover any equilibrium conformation, but must instead undergo a series of arm retraction events. In such events, the free (untethered) ends of the arms of the star must retract back along the tube which confines them (called the 'primitive path' of the arm) and then poke into a new section of tube. The stress associated with the star-arm being confined to the old tube is thus forgotten. For the entire stress to be forgotten, the arms of the star must relax back to within an entanglement length of the junction point of the star.

If the length of the arms of the star, $N$, is many times greater than the entanglement length $N_{e}$, then retraction is very unlikely. There exists an entropically generated 'thermal tension' that pulls the free ends of the arms outward to explore new conformations. Arm retraction is opposed by this thermal tension.

As the arm retracts fractional distances $s(0<s<1)$ back along its primitive path, it can be thought of as moving in a potential $U(s)$ given by Pearson and Helfand [40] as $U(s)=\frac{15 N s^{2}}{8 N_{e}}$ (in units where $k_{B} T=1$ ). When $N / N_{e}$ is large, the activation barrier for arm retraction back along its primitive path a fractional distance $s$ is many $k_{B} T$. The time $\tau(s)$ for this retraction to take place is thus exponentially long, $\tau(s)=\tau_{0} \exp [U(s)]$.

Ball and McLeish [41] added to the theory of Pearson and Helfand by realising that individual stars surrounded by other stars do not relax in a rigid environment. The arm-retraction process that one star undergoes is also exhibited by the stars surround- 


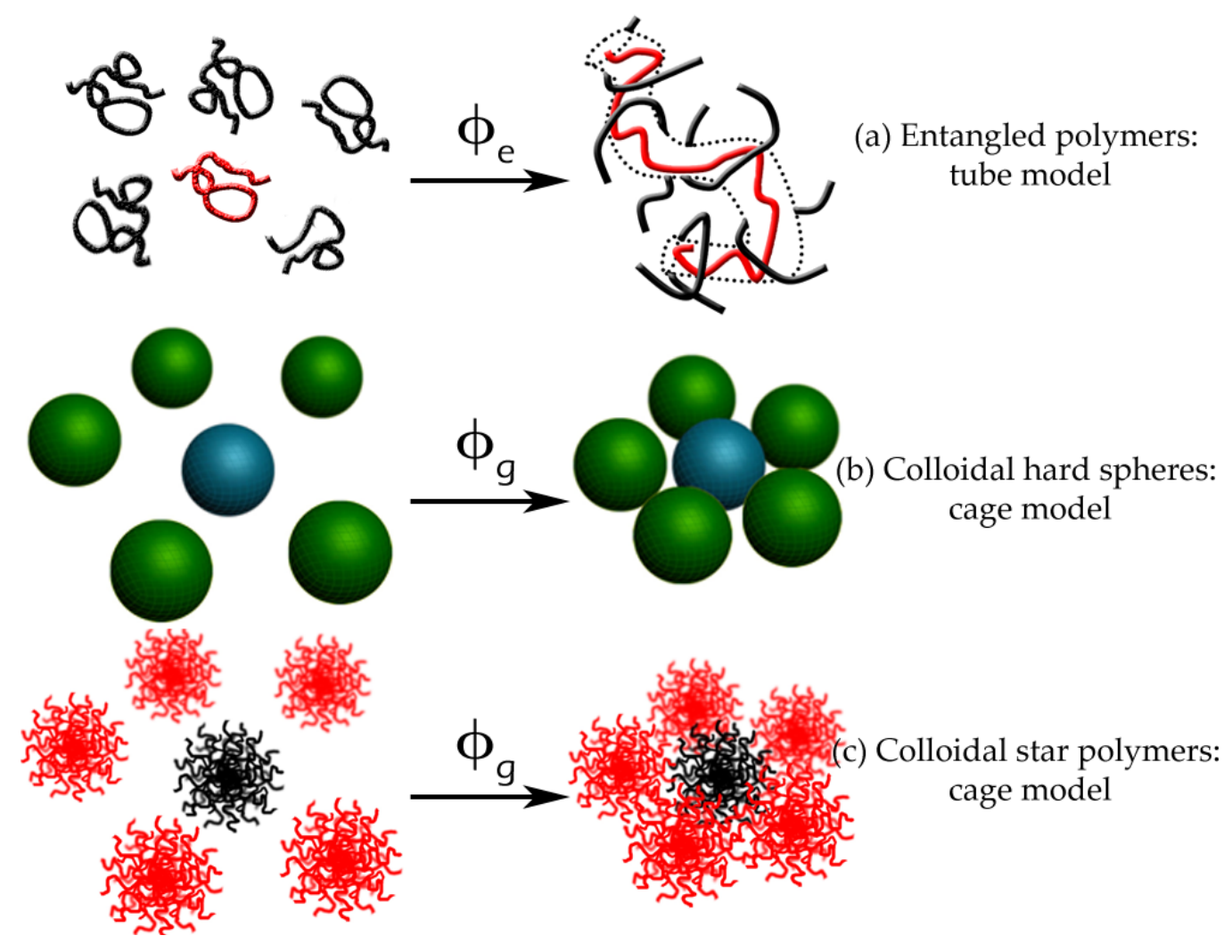

Figure 4.1: Schematic representation of crowded systems. (a) Polymers are confined to tubes at high concentrations and are said to be 'entangled' and relax via reptation. (b) Colloidal hard spheres are kinetically arrested above a volume fraction $\phi \sim 0.58$. Neighbours form effective cages which must be broken for macroscopic flow. (c) Colloidal star polymers are kinetically arrested at higher volume fractions, $\phi \sim 1.4$ than hard spheres although the same ideas of caging are applied to their macroscopic behavior. Additional to the idea of local cages is the idea of retraction which is directly analogous to reptation with one tethered end. A combination of retraction and caging must be used to fully describe the behavior of stars. 
ing it. There is thus a diluted entanglement network seen by the relaxing segments at $s$. Put simply, a portion of star arm must explore a tube made larger by dilution before relaxing by arm retraction. Ball and McLeish further assume that in such a diluted network with a volume fraction $\phi$ of chains, the entanglement length $N_{e}(\phi)$ is given by $N_{e}(\phi)=N_{e} / \phi, N_{e}(s)=N_{e} /(1-s)$.

Milner and McLeish [42] added to the theory of stress relaxation in star polymers by assuming that retraction for small $s$ such that $U_{\text {eff }}<k_{B} T$ (now an effective potential due to dilution effects), and thus also short times, is not activated but is rather that of the end of a long Rouse chain, which moves faster than diffusively. Milner and McLeish also note that Colby and Rubenstein [43] had pointed out that scaling of the entanglement length, $N_{e}(\phi)=N_{e} / \phi$ is not supported by experimental data. Colby and Rubenstein present an alternative scaling argument that leads to $N_{e}(\phi)=N_{e} / \phi^{4 / 3}$, which is supported by rheological data. The only parameters of the theory of Milner and McLeish, which are the plateau modulus $G_{0}$, the entanglement molecular weight $N_{e}$, and the Rouse time of an entanglement segment $\tau_{e}$, can be obtained from rheological data on linear chains [42].

\subsection{Ageing in soft matter}

Many soft materials exhibit relaxations on a slow time scale that are strongly reminiscent of the glassy dynamics observed in hard condensed matter. The temporal extension of these relaxations is commonly referred to as 'ageing' in analogy with the slowing down of the dynamics in ageing hard glasses and is due to the materials being far from their thermodynamical equilibrium. The continuous evolution of the mechanical and dynamical properties towards equilibrium is termed ageing and has been the focus of much research.

Ageing phenomena have been reported for a wide range of soft materials which include multilamellar vesicles [44], polymer nanocomposites [45], colloidal suspensions [46, 47, 48, 49, 29, 50], clays [51, 52, 53, 54, 55, 56, 57], pastes [58], emulsions [59], foams [60], and even in the cytoskeleton of human muscle cells [61]. Experimental techniques employed in these investigations include light scattering techniques such as diffusing wave spectroscopy [54,51], dynamic and multispeckle dynamic light scattering $[46,44,56,55,48,49]$, x-ray photon correlation spectroscopy (XPCS) [53], local rheological methods [57], conventional rheology [45, 47, 61, 44, 58, 29] and simultane- 
ous optical and rheological measurements [60]. Typical of these reports is the temporal re-scaling of relaxation dynamics with the waiting time $\left(t_{w}\right)^{\mu}$, where the ageing exponential $\mu \leq 1$, to show universality curves. The interested reader is directed to a review on this topic [62]. In [47], age-dependent shear start-up experiments have been performed on a colloidal suspension of silica particles stabilized by a layer of adsorbed polymer suspended in water. It has been shown in this case that the value the stress rises to before the onset of flow, $\sigma_{\text {plas }}$, increases with $t_{w}$, as does the time it takes the system to settle to the steady-state value $\sigma_{s s}$ which determines the steady-state flow curve. This phenomenology matches, to a certain degree, that predicted by $[47,63,64,65]$.

\subsection{Heterogeneous flow in soft materials}

Shear banding has been observed in worm-like micelles [66, 67], emulsions near their yield stress [68], entangled polymer solutions [69], liquid crystalline polymers [70], recently in a nearly random close packed, hard-sphere colloidal suspension [71] and has also been reported in dense pastes of large $(0.29 \mathrm{~mm})$ polystyrene beads [72]. Shear banding, identified experimentally through the coexistence of two or more bands of different viscosity or microstructure, is typically accounted for theoretically by a constitutive flow curve with an unstable branch of negative slope. When shear-rate conditions that correspond to the region of unstable flow are imposed, these systems can separate into phases of different local shear rates that coexist at a common stress. A recent review by Moller et al. [73] suggests shear banding and thixotropy can be viewed as two effects of the same underlying cause. The fascinating question as to whether non-monotonic constitutive behavior might be inherent to glassy yield-stress systems, remains open.

\subsection{Previous work on star polymer systems}

In [29], a two-stage evolution of the storage modulus $G^{\prime}$ is reported in the linear rheology of a colloidal glass made of stars with functionality $f=110$ and molecular weight $74100 \mathrm{~g} / \mathrm{mol}$ at two different concentrations, $c=1.6 c^{*}$ and $c=2.1 c^{*}$. The focus of the work presented herein is on suspensions of stars with functionality $f=128$ and molecular weight $80000 \mathrm{~g} / \mathrm{mol}$ and will build the non-linear rheological picture that preceded 
the two-stage evolution reported in [29]. We show the evolution from a monotonic flow curve to a flow curve that is dominated by a stress plateau and show the expected velocity profiles in concentric cylinder couette geometry.

Recent studies on high functionality star polymer [25] solutions have suggested both glassy dynamics and anomalous shear behavior [31]. In this thesis the relationship between ageing and shear banding is investigated. Star pair interactions depend strongly on their number of arms (functionality $f$ )[26] and as $f$ increases the tendency to hard sphere behavior is increased. Helgeson et al. have shown suspensions of such stars form glasses [29] while reversible gelation due to thermal swelling has been found [30] in suspensions of these stars. Such properties suggest hitherto unknown behaviors that challenge conventional models of glassy behavior. Furthermore, the soft stars studied here provide one of the few examples of soft matter in which the glass transition can be easily crossed via temperature and not volume fraction. In order to relate heterogeneous flow to the time dependent stress/strain-rate constitutive properties, an experimental technique should combine rheo-mechanics with non-invasive velocimetry. In our approach time-resolved nuclear magnetic resonance (NMR) microscopy is the velocimetry of choice.

The main contribution of the present work is to perform the first experimental study of the interplay of ageing and shear banding in a soft material where a distinct 2-step evolution is seen. Rheological and rheo-NMR measurements on a system of star polymers with nominal functionality $f$, of 128 arms are reported, each having nominal molecular mass, $M_{a}$, of $80000 \mathrm{~g} / \mathrm{mol}$ at a temperature of $293 \mathrm{~K}$ and at a concentration of $c=2 c^{*}$. Such stars exhibit behaviors intermediate between linear polymers and hard spheres, with evidence of both caging effects and soft interactions of the type associated with polymer entanglement. The current system therefore assists in elucidating how soft interactions that are not present in many hard-sphere systems manifest themselves on a macroscopic scale. An earlier Rheo-NMR study by Holmes et al [31] indicated intermittent shear rate heterogeneity. However that work, while on the same star polymer, was for a different solvent and at lower concentration, closer to $c^{*}$. It utilized a wide gap Couette cell with correspondingly wide stress variation across the gap, and used pre-shear protocols that differed in the case of the NMR and rheo-mechanical data. We here report on dynamics, involving both ageing effects and shear banding, in the $c=2 c^{*}$ star polymer in squalene, for which consistent protocols are used and in which the Rheo-NMR experiments are performed in a much narrower 
gap cell. 


\section{Chapter 5}

\section{Experimental: Conventional Rheology}

\subsection{Introduction}

The ageing dynamics of the soft glass that are investigated in this thesis are highly dependent on the shear history. A detailed analysis of the mechanical and dynamical changes due to ageing thus requires a reproducible, fully rejuvenated initial state. Thus the first tests reported focus on determining the conditions under which a sample can be fluidized, or "rejuvenated", and the response to oscillatory shear in the linear regime directly after fluidisation. Once this protocol is determined, transient non-linear tests are performed, the results of which show evidence of a stress plateau in the flow curves at low shear rates and long times of two 128-arm samples at two different concentrations. The two concentrations reported here are $c=1.5 c^{*}$ and $c=2 c^{*}$, where $c^{*}$ is the overlap concentration and is defined as $c^{*}=\frac{3 f M_{a}}{4 \pi N_{a} R_{H}^{3}}$ where $N_{A}$ is Avogadro's number, $M_{a}$ is the molecular weight of each arm and $R_{H}$ is the equivalent hard-sphere radius of the polymer coil.

Rheological measurements presented here were performed on an ARES-HR straincontrolled rheometer fitted with cone-plate geometry of diameter $25 \mathrm{~mm}$, angle $0.04 \mathrm{rad}$ and a truncation of $48 \mu \mathrm{m}$. To ensure a saturated atmosphere and one on which air currents have negligible effects, a trap system containing mats soaked with squalene surrounded the geometry. All experiments were carried out at $293 \mathrm{~K}, 20^{\circ} \mathrm{C}$ 


\subsection{Determining the conditions for rejuvenation: Estab- lishing a protocol}

When determining the properties of materials as they age, it is paramount that there exists a way to determine the age of a sample and 'reset' it. This requires a knowledge of how the material behaves in its fully rejuvenated state, hereafter referred to as the 'youthful state' and an ability to reverse any ageing that takes place. The 'youthful state' of the material refers to the response shortly after such a rejuvenation. In the early years of work on soft glasses, the resetting of physical ageing was often referred to as a 'quench'. The term 'quench' is a layover from the language of hard glasses, where, to bring the glass far from thermodynamic equilibrium, the temperature of the material was lowered quickly after a high-temperature fluidization. In the case of soft glasses, it is possible to fluidise a sample via mechanical means. We comment here that recent simulation work by Isner and Lacks [74] and a review of experimental work by McKenna [75] suggest a distinction be made between mechanical 'rejuvenation' and thermal quenching. It is conjectured that large mechanical stimuli do not entirely rejuvenate or erase all effects of ageing. However, a reproducible shear protocol with a large amplitude oscillatory shear or steady shear with large rate that takes the sample well in the shear-thinning regime might at least bring the sample reproducibly to a certain state. The precise meaning of 'shortly' changes for each material. For many soft materials the ageing is seen as a stiffening or partial solidification. This can often be 'undone' by a simple addition of energy via shear and/or heating to re-fluidize the entire sample. An application of large amplitude oscillatory shearing ensures an identical state is reached before each experiment and the conditions for such a rejuvenation need to be determined before any meaningful ageing experimentation can be conducted.

\subsubsection{Nonlinear Strain Response to Oscillatory Shear}

An experiment where an oscillatory strain is applied at a constant angular frequency while the strain is swept over many orders of magnitude is designed to establish the limits of the linear and nonlinear regimes by way of tracking the dynamic moduli. Such a test was carried out on a fully aged sample of 12880 stars in squalene at a concentration of $c=2 c^{*}$ at a frequency of $1 \mathrm{rads}^{-1}$ to help formulate a preshearing 
protocol (Fig. 5.1(a)). At small strains $G^{\prime}$ is almost ten times larger than $G^{\prime \prime}$ and both are relatively constant, indicating the material behaves as a viscoelastic solid under these conditions. The strain that separates the linear regime from the non-linear at this frequency is approximately $2.5 \%$. As the amount of deformation is increased, $G^{\prime \prime}$ goes through a maximum value suggesting a breaking of cages at the onset of flow. The point where $G^{\prime \prime}$ reaches a maximum and crosses $G^{\prime}$ indicates a yield strain of $\sim 10 \%$. Above the crossover point the sample is flowing, which is our condition for rejuvenation. The large amplitude, shear-thinning behaviour of $G^{\prime}$ and $G^{\prime \prime}$ were fit with a power law. The fit yields thinning exponents for $G^{\prime}$ of $1.328 \pm 0.001$ and $G^{\prime \prime}$ of $0.632 \pm 0.001$. The values are lower than those expected by a recent approximate extension of MCT to nonlinear rheology, [76] and the ratio of the two, within experimental uncertainty, is higher. This result agrees with a finding in PNIPAM microgels [77]. The reasons for this may include the assumption that the contribution of higher harmonics in the Fourier spectrum is negligible [76]. Similar results are obtained for $c=1.5 c^{*}$. It follows that a successful preshear will be achieved by applying an oscillatory strain at $1 \mathrm{rad} \mathrm{s}^{-1}$ with amplitude $100 \%$. Such conditions were applied for an arbitrary time of $200 s$ by way of a constant amplitude and frequency oscillatory strain experiment for all tests.

These conditions establish a protocol for resetting the age of the system for the start of each test. It was determined empirically that after this preshear the material gave reproducible mechanical responses. We use the end of this preshear as the definition of the origin of time for all experiments presented herein. For all experiments where ageing effects are important there is a useful convention which will be used throughout this work. While the absolute time, $t$ is reset directly after rejuvenation, it will be necessary to let the system evolve spontaneously for a time $t_{w}$, traditionally called the waiting time, before applying stresses or strains. We then define the experimental time as $t^{\prime}=t-t_{w}$.

Having found conditions that place the sample in a reproducible fluid state, the frequency response of the youthful material in the linear regime is determined as a benchmark for all other ageing experiments. The determination of the youthful response is achieved by applying an oscillatory shear of constant amplitude of $1 \%$ and varying the frequency while recording the storage and loss moduli. The results of such a test are displayed in Fig. 5.1(b). The storage modulus shows a very weak power-law type dependence $G^{\prime} \propto \omega^{0.087 \pm 0.003}$ over the three orders of magnitude. At frequencies $\omega<0.5 \mathrm{rads}^{-1} G^{\prime \prime} \propto \omega^{-0.19 \pm 0.05}$. For frequencies $1 \mathrm{rads}^{-1}>\omega>10 \mathrm{rads}^{-1}$ the 

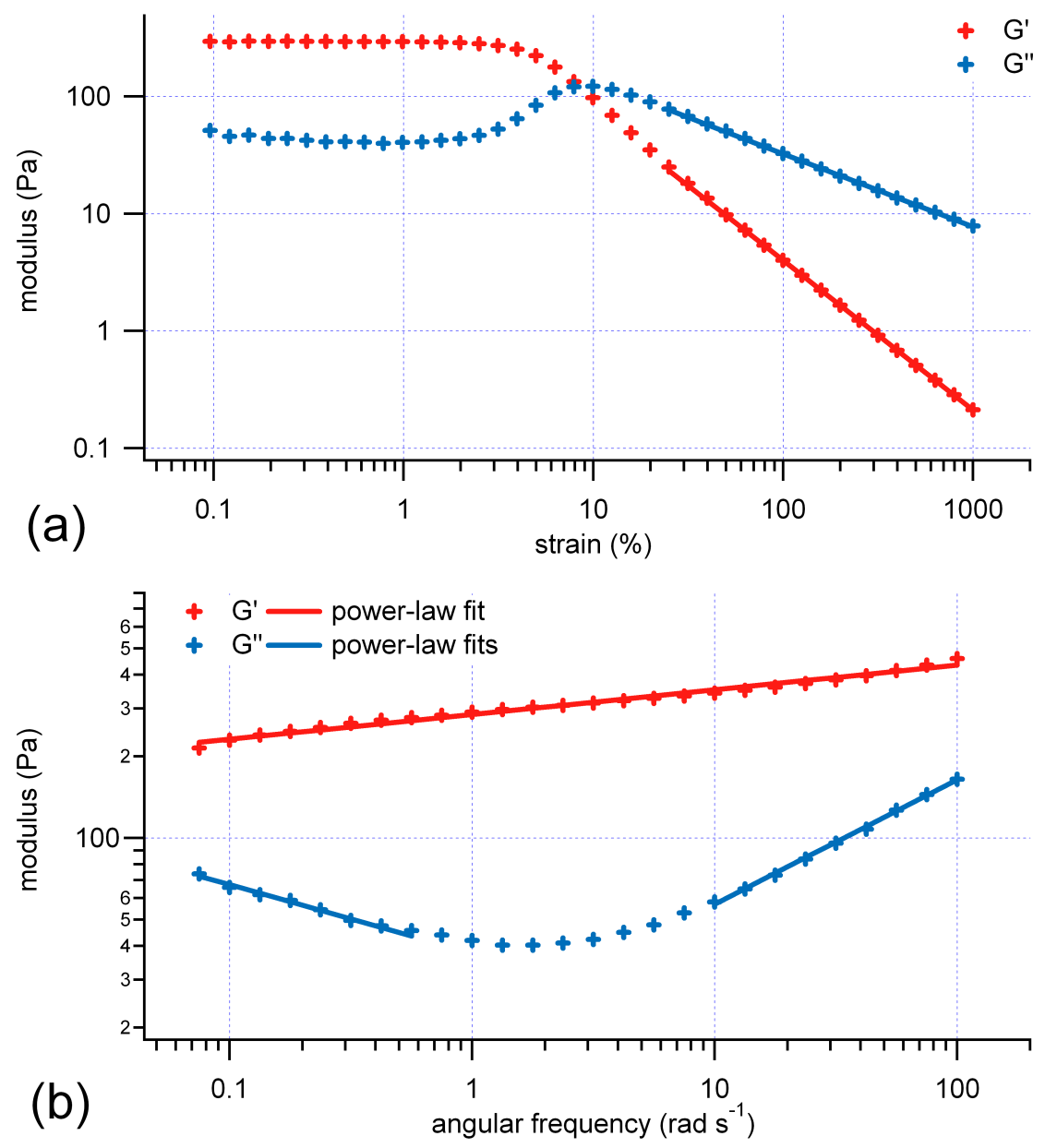

Figure 5.1: (a) A log-log plot of the strain amplitude dependence of the storage and loss moduli, $G^{\prime}$ and $G^{\prime \prime}$ at $1 \mathrm{rad} \mathrm{s}^{-1}$ for a sample of stars in squalene at $c=2 c^{*}$. At low amplitudes the material behaves linearly and solid-like. As the deformation increases, the moduli cross over and the material shows a shear-thinning liquid response at amplitudes above $10 \%$. Solid lines represent asymptotic predictions for hard spheres. (b) Log-log plot of the frequency dependence of $G^{\prime}$ and $G^{\prime \prime}$ at a fixed strain amplitude of $1 \%$ directly after rejuvenation. The storage modulus shows a weak power-law dependence and $G^{\prime \prime}$ shows a minimum typical of glassy colloidal systems. This non-aged, solid-like behaviour is the benchmark for further investigations. Similar results are found for stars in squalene at $c=1.5 c^{*}$. 
power-law exponent describing the loss modulus increases and changes sign so that for frequencies $\omega \geq 10 \mathrm{rad} \mathrm{s}^{-1}, G^{\prime \prime} \propto \omega^{0.49 \pm 0.02}$.

These responses, and others to follow are very similar in nature to those seen by Derec et al. [47] in their investigation of the rheology of PEO-protected silica particles, by Mason and Weitz [78] in their investigation of uncoated silica spheres in ethylene glycol and by Helgeson et al. [29] in their study of stars similar to those of this work. The wide variety of systems that show similar behaviours indicates a potential universality.

\subsection{Transient response to continuous steady shear}

The transient response of the material to continuous steady shear at various rates with various waiting times was investigated. In order to examine the response of the material without filtering from the software controlling the rheometer, direct measurements of the output voltage of the rheometer were made. The TORQUE OUT and STRAIN/NORMAL OUT connectors of an ARES strain control rheometer were connected to an ADC and a PC as described by Wilhelm et al. in [57]. The TORQUE OUT channel of the rheometer outputs a DC voltage that is proportional to the transducer torque. When in dynamic mode, the STRAIN/NORMAL OUT channel outputs a voltage that is proportional to the actual motor angular deflection and when in steady mode it outputs a voltage that is proportional to the transducer normal force. LabVIEW ${ }^{\mathrm{TM}}$ Software was used to access the raw torque/strain data from the ADC while RSI Orchestrator was used to control the rheometer in the usual way.

A series of start-up flow measurements in the cone-and-plate geometry were made. The system was presheared according to the established protocol and then allowed to evolve spontaneously for a time $t_{w}$ before the application of a constant shear rate. The waiting times were varied from $0 s$ to $2500 \mathrm{~s}$. The stress was measured as a function of the experimental time $t^{\prime}=t-t_{w}$.

Of primary note is the reported stress at very short times. Orchestrator reports that the stress always starts at zero, regardless of waiting time. This suggests that any internal stress left in the sample from the mechanical fluidification protocol dissipates instantaneously. In practice, the time control of the rheometer has a finite time between finishing the oscillatory rejuvenation and the application of a continuous, steady shear rate, which will henceforth be referred to as $0^{*}$, and so any internal stresses must dis- 


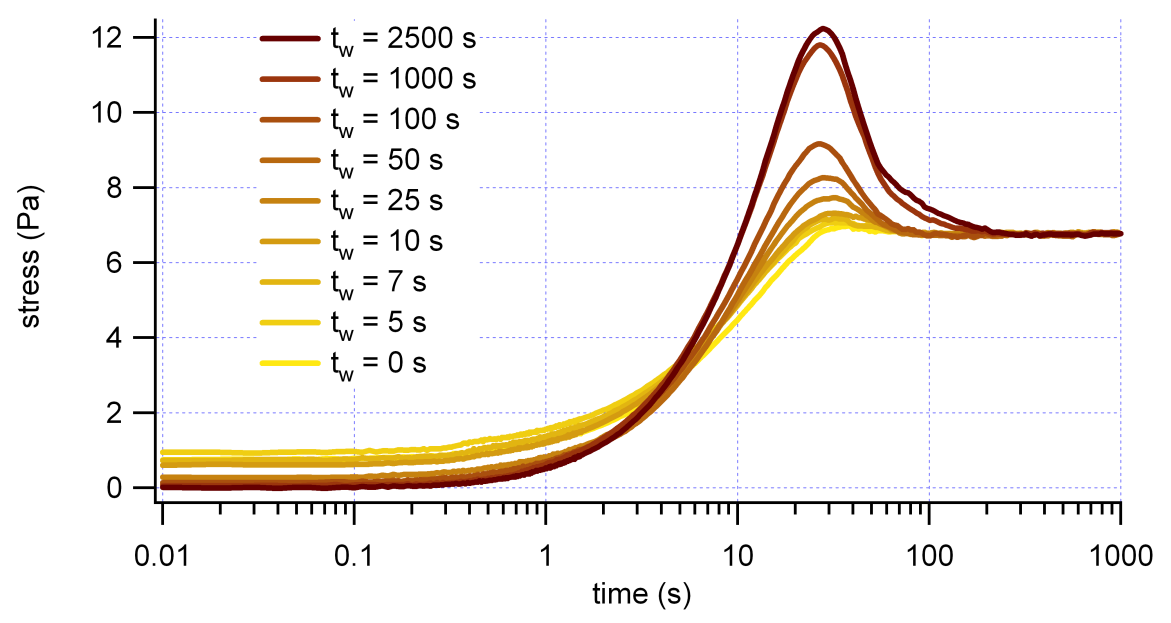

Figure 5.2: Shear startup for star concentration of $2 c^{*}$ and $\dot{\gamma}=0.0025 \mathrm{~s}^{-1}$ in the cone-and-plate geometry: Lin-log plots of the stress response as reported by RSI Orchestrator adjusted to make the stresses at $t^{\prime}=1000 \mathrm{~s}$ equate for waiting times $t_{w}=0^{*}, 5,7,10,25,50,100,500,1000$ and $2500 \mathrm{~s}$. Any disparities due to the software setting the initial stress to zero have been eliminated.

sipate in a maximum of that time in order for the Orchestrator-reported stresses to be true. Contrary to the stresses reported by Orchestrator, the TORQUE OUT channel of the rheometer reports non-zero stresses at $t^{\prime}=0$ for $t_{w} \geq 500 \mathrm{~s}$. A non-zero stress at short times suggests there is a significant amount of internal stress left in the system by the mechanical rejuvenation protocol that takes between 50 and $500 s$ to dissipate.

The stress evolution reported by RSI Orchestrator can be adjusted so the stresses reported at $t^{\prime}=1000 \mathrm{~s}$ equate. The results of such adjustments are displayed in Fig. 5.2 for an applied shear rate of $\dot{\gamma}=0.0025 \mathrm{~s}^{-1}$. Any disparities at long times reported by Orchestrator caused by the setting of the initial stress to zero have been eliminated.

The values of the initial stress and stress overshoots shown in Fig. 5.2 are displayed in Fig. 5.3. The stress residual from the preshear decays with waiting time as a power law with exponent $-0.57 \pm 0.05$. Similar results to [47] are found for the plastic overshoot: the older the sample is, the more stress it stores before flowing. The stress at the plastic overshoot increases with waiting time with a power-law exponent $0.096 \pm 0.005$ for waiting times $t_{w} \leq 2500 \mathrm{~s}$. Like the results in [47], the data presented here also suggest that the time/strain at which rearrangements take place and flow begins is not a function of $t_{w}$.

The stress response to a step-wise application of a constant shear rate follows three 

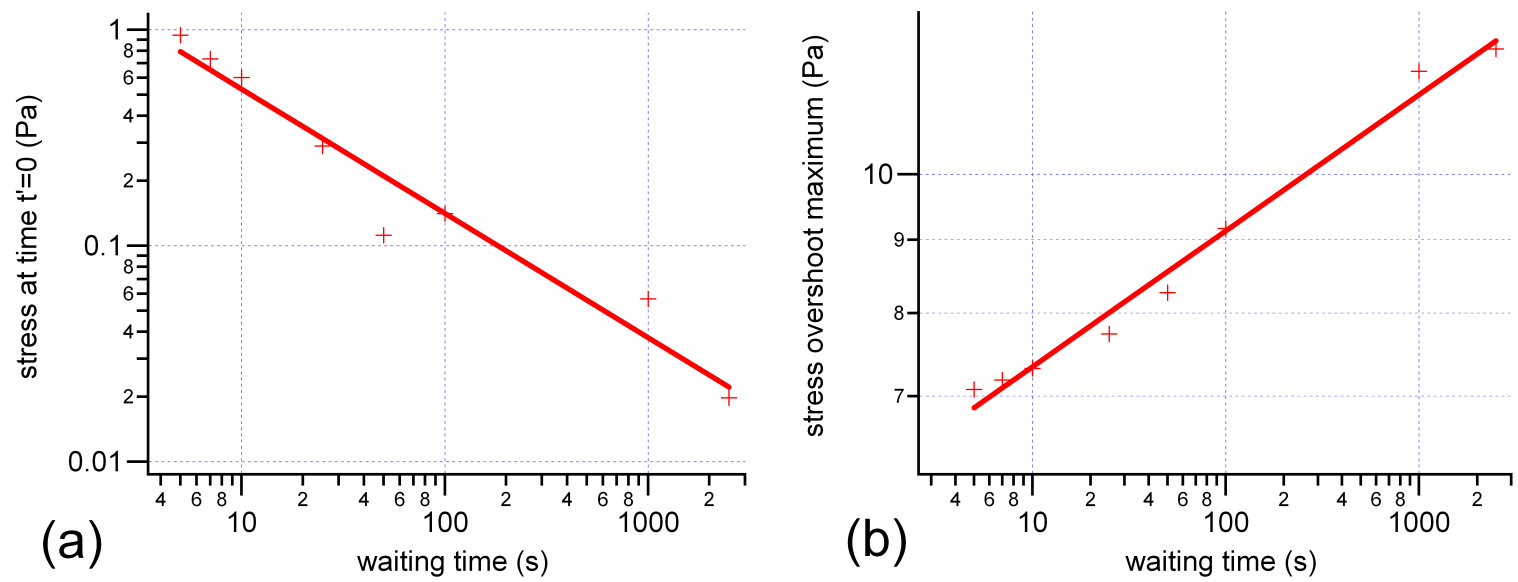

Figure 5.3: (a) The stress residual from the preshear (points) decays with a power-law exponent $-0.57 \pm 0.05$ (solid line). (b) The maximum stress achieved at the plastic overshoot (points) increases as a power-law with exponent $0.096 \pm 0.005$ (solid line).

distinctly different behaviours as exhibited by Figs. 5.4 and 5.5. The waiting time is kept constant at a value of $t_{w}=0^{*}$ and a stress corresponding to $t_{w}=0^{*}$ found previously is added to the Orchestrator-reported data. The response of the material to shear rates greater than the critical rate of $0.08 s^{-1}$ in the $c=1.5 c^{*}$ sample is as follows: initially the stress rises linearly before reaching a maximum plastic overshoot value at the onset of flow. Once flowing, the stress then settles to a stable value for all time. For shear rates less than $0.05 \mathrm{~s}^{-1}$ and greater than $0.0025 \mathrm{~s}^{-1}$ there is a similar stress overshoot followed by an interval of steady stress response. We refer to this period as 'adolescence'. As the time under constant shearing reaches $\sim 2000 s$ for all rates less than $0.05 s^{-1}$, the stress rises and appears to overshoot again before settling to a value around $8 \pm 0.25 \mathrm{~Pa}$. The third type of behaviour observed is that of the material when subjected to shear rates less than or equal to $0.0025 \mathrm{~s}^{-1}$. At shear rates equal to or below this value, there is no initial stress overshoot as the stress rises to its adolescent value, where it stays for a time before exhibiting the same rise and overshoot to a stress of $8 \pm 0.25 P a$.

The critical shear rates and stress values are concentration dependent. In the $c=2 c^{*}$ sample, the higher stressed regime occurs at a stress of $11 \pm 0.25 \mathrm{~Pa}$ and when the concentration is $1.5 c^{*}$ it occurs at a stress of $8 \pm 0.25 \mathrm{~Pa}$. In the $c=2 c^{*}$ sample, the critical shear rate below which there is no increase in stress at long times is $0.035 \mathrm{~s}^{-1}$ 

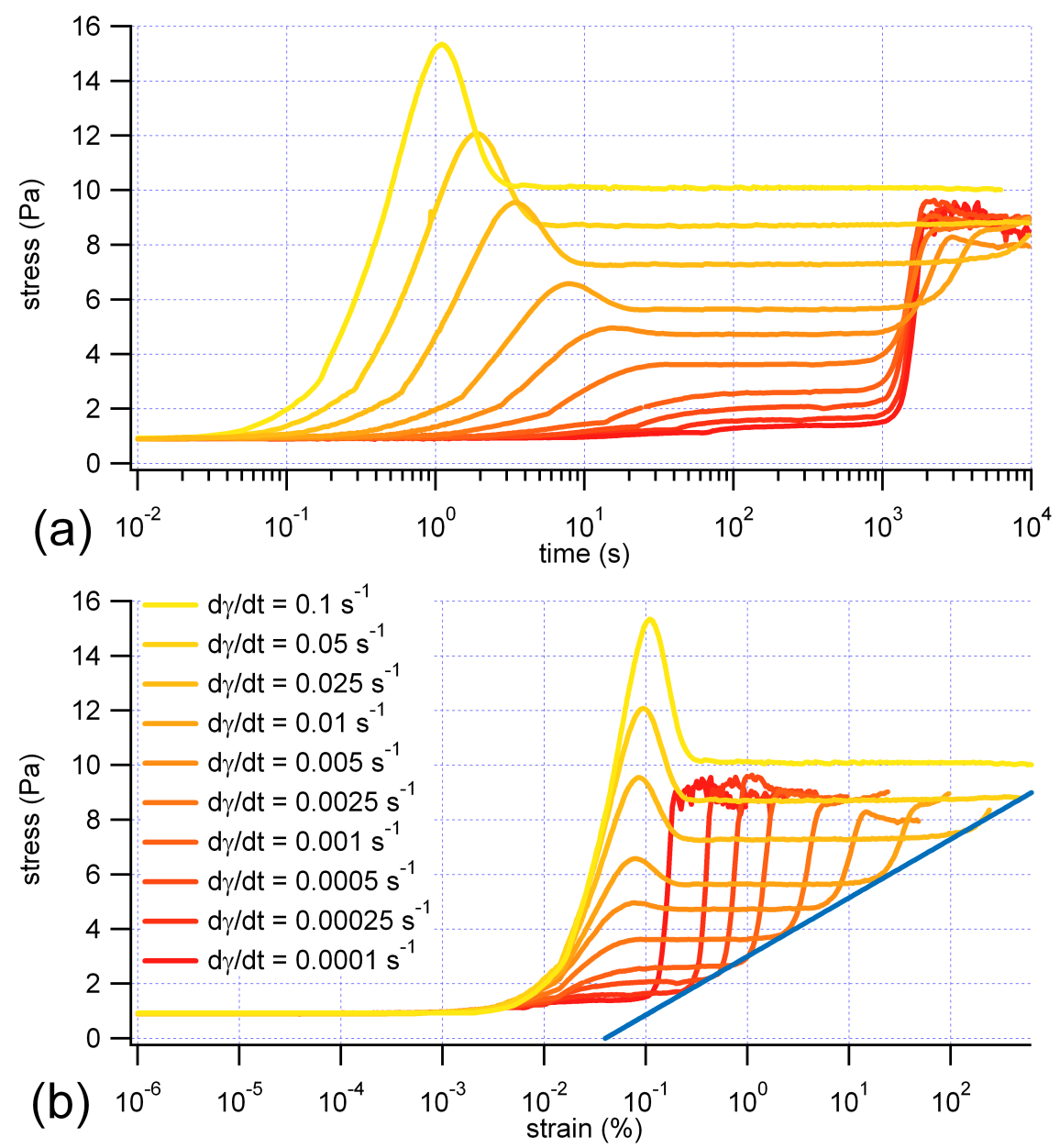

Figure 5.4: Shear startup, $t_{w}=0^{*}$ for star concentration of $c=1.5 c^{*}$ in the cone-and-plate geometry: Note the three types of shear rate-dependent response: an overshoot with no stress increase at long times (continual adolescence); an overshoot followed by a steady adolescent response and then stress increase; no overshoot with stress increase at long times. The strain of the stress overshoot shown in (b) is an increasing function of strain rate. The strain at which the small kink, visible just above a strain of $10^{-2}$ at all rates, is also an increasing function of strain rate. The solid blue line is a logarithmic fit to the stress increase. 

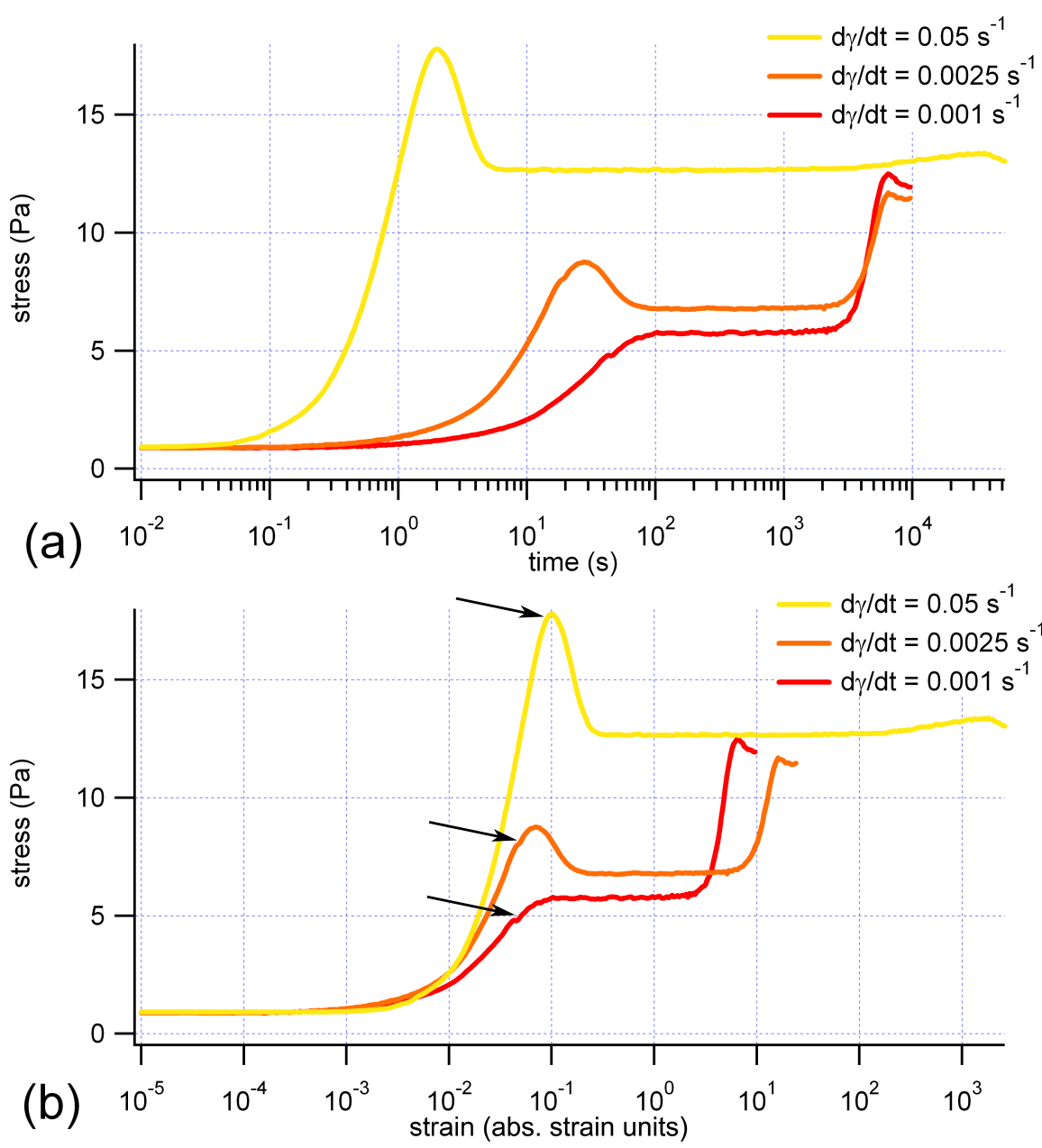

Figure 5.5: Shear startup for star concentration of $2 c^{*}$ in the cone-and-plate geometry: Lin-log plots of the $t_{w}=0^{*}$ stress response as a function of time (a) and strain (b) to three steady shear rates after preshear. The same features as seen in the data of Fig. 5.4 are evident though the plateau occurs at a higher stress. Note the small kink visible at strains of $\sim 4.6 \%$ as indicated by the arrows in (b). This feature is clearer at the two lower rates than in the higher and, like the strain position of the stress overshoot, occurs at larger strains for higher rates. 
(as determined from the flow curve displayed in Fig. 5.8, shown in the next section) whereas in the $c=1.5 c^{*}$ case it is $0.05 \mathrm{~s}^{-1}$. The rate below which no initial stress overshoot occurs is $0.001 s^{-1}$ at $c=2 c *$ and $0.0025 s^{-1}$ when the concentration is $1.5 c^{*}$.

There is a small non-linearity in the stress response at a strain of $\sim 1.1 \%$ in the $c=1.5 c^{*}$ case and at a strain of $\sim 4.6 \%$ in the $c=2 c^{*}$ case at all rates. The similar strain-alignment of these features alludes to a structural rearrangement. In the $c=1.5 c^{*}$ case, the non-linearity is seen as a change in the rate at which the stress increases at a strain of $\sim 1.1 \%$. In the $c=2 c^{*}$ case, the non-linearity is manifested as a dip in the stress response at strains $\sim 4.6 \%$. For both concentrations, the nonlinearity occurs at smaller strains for lower rates than at higher rates. This is related to the increasing strain at which the stress overshoot maximum occurs, which is indicative of the material's ability to acquire more strain at higher rates before yielding and beginning to flow. This rate-dependent yield strain is supported by the oscillatory data of Helgeson et al. [29] for two systems of identical stars at concentrations of $c=1.5$ and $2.1 c^{*}$. The relationship between shear rate and the absolute strain at which the nonlinear inflection and stress overshoot occur in the $c=1.5 c^{*}$ sample is displayed in Fig. 5.6. From the data of Fig. 5.5(b) it can be deduced that cages break at strains of $\sim 10 \%$ for rates $\dot{\gamma} \leq 0.1 \mathrm{~s}^{-1}$. The data of Fig. 5.1 support this and also suggest the small non-linearities correspond to a regime between the onset of nonlinear flow and shear melting.

The difference between the maximum stress achieved in the initial overshoot and the adolescent stress increases with shear rate. Derec et al. [47] comment on the size of the stress overshoot, $\sigma_{\text {plastic }}$ in their study of a suspension of silica spheres: "the smaller the inverse of the strain rate compared to some intrinsic time characteristic of rearrangement in the system, the larger the stress stored before rearrangements occur." This condition indicates some intrinsic time characteristic of $\sim 400 \mathrm{~s}$ for the $c=1.5 c^{*}$ sample and $\sim 1000 s$ in the $c=2 c^{*}$ sample corresponding to the inverse of the largest shear rate at which no overshoot is seen in each case.

The response of the material in the regime of higher stresses is less stable and fluctuates in time, indicating a possible transition to an agglomerated state where larger clusters are in a continual state of breakup and renewal. Derec et al. [47] measured for $10^{3} s$ to check for steady state with waiting times up to $10^{4} s$ with no evidence of any stress plateau. The system studied by Derec et al. [47] is a suspension of silica particles stabilized by a $7 \mathrm{~nm}$ layer of PEO on the surface of each particle. The phenomeno- 


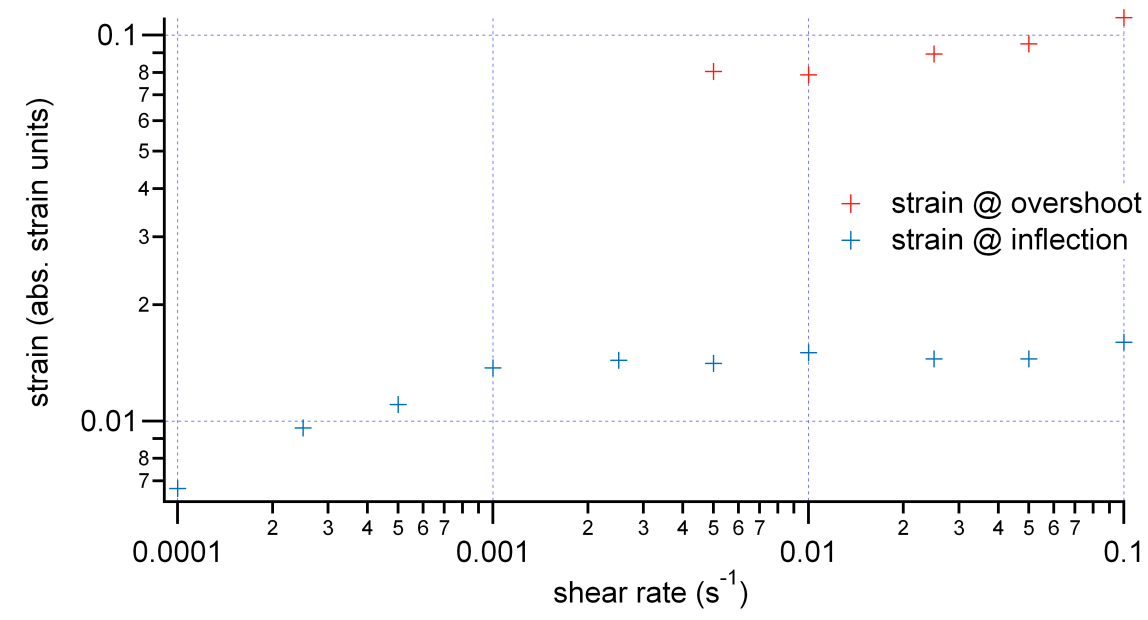

Figure 5.6: The absolute strains of the nonlinear inflection and stress overshoot maximum as a function of shear rate for the $c=1.5 c^{*}$ sample. The strain at which the nonlinear inflection occurs is constant for shear rates of two orders of magnitude from $\dot{\gamma}=0.001 \rightarrow 0.1$. The strain at which the stress overshoot maximum occurs is an increasing function of shear rate for the rates at which an overshoot is seen.

logical response in both the linear and non-linear rheological regimes of the stars is reminiscent of the response of Derec et al.ś [47] suspension of stabilized silica particles with the exception of the slow evolution of the stress plateau. That latter behaviour is a particular feature of the star polymer system, though stress plateaux have been previously seen in many other systems. See for example $[79,80,66,67,81,82,68,69,70]$ for investigations into a suspension of nearly equal-sized colloidal hard spheres, the worm-like surfactant solution cetylpyridinium chloride/sodium salicylate $100 \mathrm{mM} / 60$ $\mathrm{mM}$, the worm-like micelle system $10 \% \mathrm{w} / \mathrm{v}$ cetylpyridinium chloride and sodium salicylate (molar ratio 2:1) in $0.5 \mathrm{M}^{2} \mathrm{H}_{2} \mathrm{O} \mathrm{NaCl}$ brine, suspensions of the synthetic clay bentonite and water at two different solid fractions, and white-cement (Ciment Super Blanc, Calcia, France) (water to solid weight ratio $=0.5$ ). This slow evolution could be an indication that the delicate interactions of the arms of the stars are responsible for the long-time behaviour while for short times the behaviour of the star suspension is dominated by the star's soft sphere-like properties.

The transition to the higher stressed regime and the strain at which maximum stress is reached in the initial overshoot are logarithmic functions of the strain as indicated by the data of Figs. 5.4 and 5.5. 


\subsubsection{The Flow Curves}

The long-time stress response to steady shear rates indicates the existence of a transition to a higher stress state for all shear rates below a critical shear rate $\gamma_{c}$ where $\gamma_{c}$ is a function of concentration. As an exhibition of this behaviour in more general terms, the flow curves (i.e. the steady-state stress response as a function of applied shear rate) for the two concentrations were measured. The beginning and end states of the evolution of the $c=1.5 c^{*}$ case are shown in Fig. 5.7 and the evolution of the $c=2 c^{*}$ sample is shown in Fig. 5.8. The data displayed in Fig. 5.8 were measured using different waiting protocols and therefore indicate the general behaviour at different parts of the stress evolution of the material. In all cases the material was mechanically fluidized and a decreasing succession of shear rates was applied, starting at $100 \mathrm{~s}^{-1}$. All of the data shown in yellow in Fig. 5.8 represent the stress averaged over a $10 s$ interval after a waiting time of $10 s$ at each shear rate. Only the data above $0.05 \mathrm{~s}^{-1}$ in the $c=2 c^{*}$ case and $0.05 s^{-1}$ in the $c=1.5 c^{*}$ case, shown in Fig. 5.7(b) were acquired this way. For all other rates, the waiting time was at least $5000 s$ for both concentrations (for rates below $0.003 \mathrm{~s}^{-1}$ ) and at most $30000 s$ (for $0.025 \mathrm{~s}^{-1}$ in the $c=2 c^{*}$ case). The time over which the stress was averaged was also increased to $1000 s$ to accommodate for the unsteady nature of the higher stressed regime. Thus, the yellow points of Fig. 5.7 and Fig. 5.8(b) should be thought of as rheograms of the adolescent states of the materials. At the lower shear rates in these youthful representations, the stress sampled is the leading edge of the initial increase to the pseudo-steady state stress and cannot be thought of as steady-state in any way. The evolution of the flow curves displayed in Figs. 5.8 and 5.7 reflect the interplay between shear effects and ageing. The rate of ageing slows as the shear rate is increased so that higher shear rates lead to slower ageing. Figure 5.7 shows an incomplete evolution where a slight minimum is still evident. The disappearance of the apparent minimum and subsequent increase of viscosity as the shear rate drops is thus the effect of this interplay and not of a bifurcation of viscosity as suggested by Bonn et al. [83] in studies on the synthetic clay Laponite. The stress plateaux of Figs. 5.7 and 5.8 indicate the possibility of shear banding at both concentrations measured. The lower branches of the flow curves, which would give a value to $\dot{\gamma}_{l}$, the lower shear rate in the banded structure, must be at least lower than $2 \times 10^{-4} s^{-1}$ for both concentrations as indicated by the lowest shear-rate measurements possible with the rheometer. 


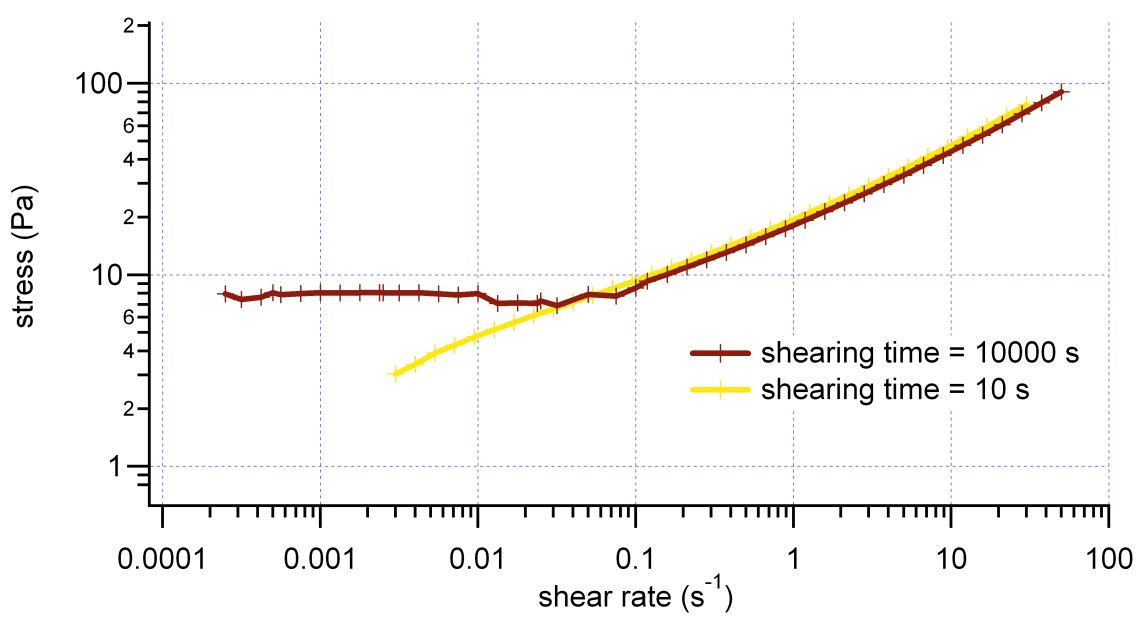

Figure 5.7: Flow curve for a star concentration of $1.5 c^{*}$ in the cone-and-plate geometry: Log-log plots of the steady state stress as a function of applied steady shear rate. (yellow) Each datum is acquired after shearing for a time $t=10 \mathrm{~s}$ after rejuvenation. (dark red) Each datum is acquired after shearing for time on the order of $10^{4} \mathrm{~s}$ to reach a steady state. The plateau clearly visible in the long time limit is thus easily missed in a fast measurement. The lines are guides to the eye.

Figure 5.9 is a generic stress transient which includes definitions to clarify a discussion of the salient points of this investigation. When strained continuously, the stress response $\sigma(t)$ first increases proportionally to the strain and then overshoots to a value $\sigma_{\text {plas }}$. Once $\sigma(t)=\sigma_{\text {plas }}$, i.e. the system has been deformed plastically, the system is forced to flow and the stress relaxes to its pseudo-steady-state value $\sigma_{p-s}$. After a period of time, the stress increases to a common critical stress, $\sigma=\sigma_{c}$. We note that all three of these stress definitions are concentration-dependent and two of these stresses are also shear rate dependent, that is, $\sigma_{p l a s}=\sigma_{p l a s}(c, \dot{\gamma}), \sigma_{p-s}=\sigma_{p-s}(c, \dot{\gamma})$ and $\dot{\gamma}_{c}=\dot{\gamma}_{c}(c)$. We use these stresses to define two critical shear rates. We define $\dot{\gamma}_{\beta}$ as the shear rate at which $\sigma_{\text {plas }}=\sigma_{p-s}$, that is, the highest rate where the stress does not overshoot initially. See, for example, $\dot{\gamma}=0.001 \mathrm{~s}^{-1}$ in Fig. 5.5. We also define $\dot{\gamma}_{c}$ as being the shear rate at which $\sigma_{p-s}=\sigma_{c}$, that is, the lowest rate where a transition to a second stress state is not observed. This latter definition is equivalent to defining $\dot{\gamma}_{c}$ as being the highest shear rate on the stress plateau as seen in Figs. 5.8 and 5.7. For rates $\dot{\gamma}>\dot{\gamma}_{c}$, where there is no transition to a second stress state, we simply refer to the steady state value of the stress $\sigma_{s-s}$.

To paraphrase: the stress evolution is found to be rate-dependent and yields one of 

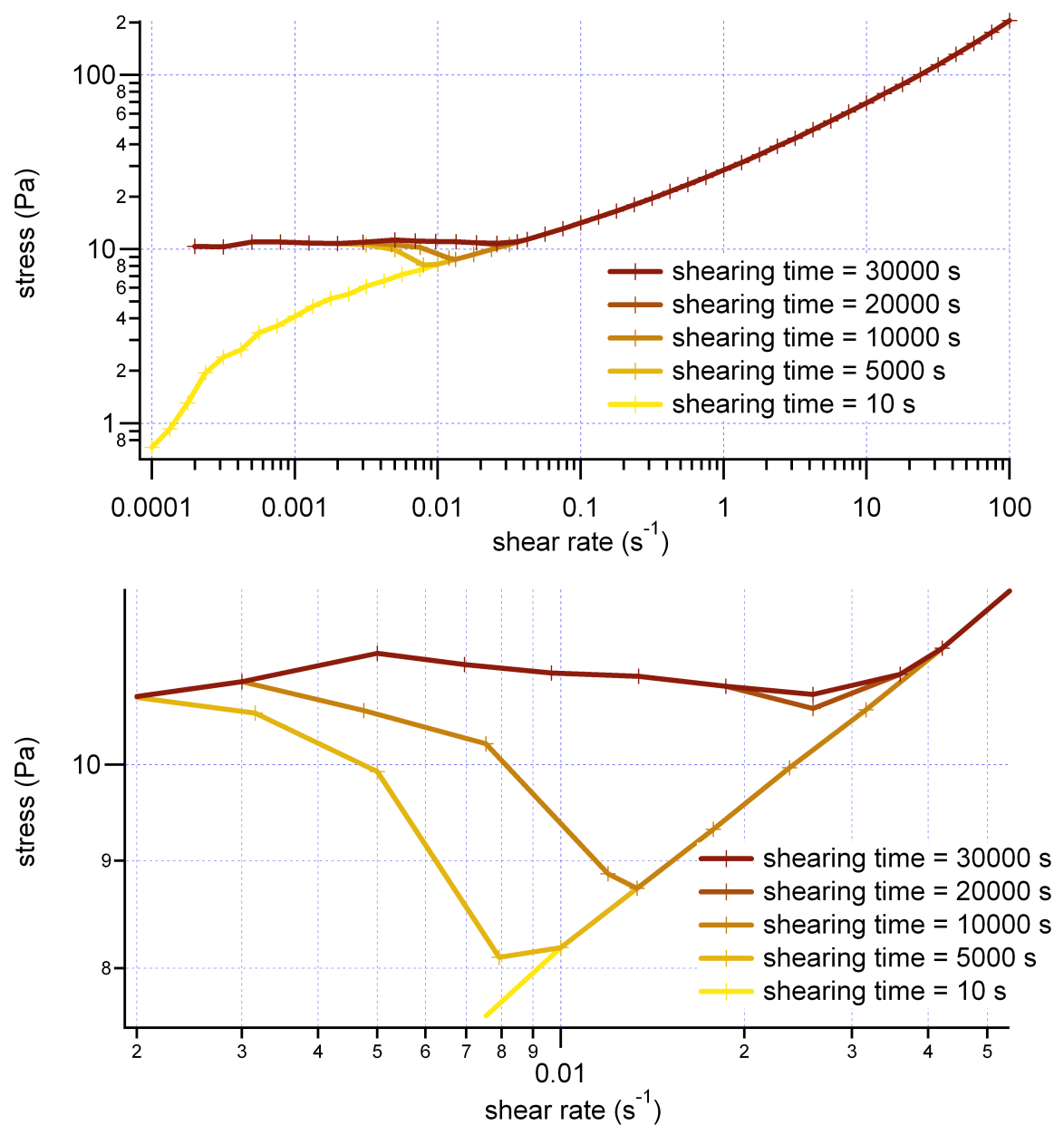

Figure 5.8: Flow curve evolution for star concentration of $2 c^{*}$ in the cone-and-plate geometry: Log-log plots of the stress as a function of applied steady shear rate for different ages of the sample. Each datum is an average over $O(100) s$ acquired after shearing for a time indicated in the plot. Note the evolution of the plateau from lower shear rates towards higher rates. For clarity, a closeup of the critical region is displayed. 


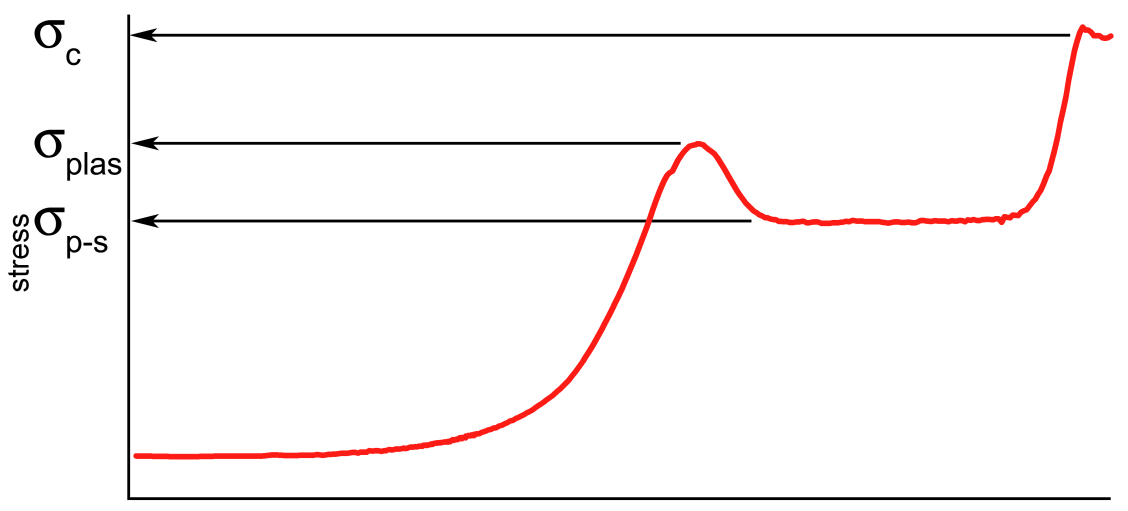

time $\left(t^{\prime}\right)$

Figure 5.9: Definitions of the key stresses involved in a full description of the transient response to continuous strain.

three typical behaviours:

- for rates $\dot{\gamma}<\dot{\gamma}_{\beta}$, the stress rises to a pseudo-steady value $\sigma_{p-s}$ and remains at this value for a time on the order of $1000 s$ before rising to a common critical stress $\sigma_{c}$

- for rates $\dot{\gamma}_{\beta}<\dot{\gamma}<\dot{\gamma}_{c}$, the stress rises and overshoots to $\sigma_{\text {plas }}$ before relaxing back to $\sigma_{p-s}$. Further, $\sigma_{\text {plas }}-\sigma_{p-s}$ increases as $\dot{\gamma}-\dot{\gamma}_{\beta}$ increases. The stress remains at $\sigma_{p-s}$ for time on the order of $1000 s$ before rising to to the common critical stress $\sigma_{c}$

- for rates $\dot{\gamma}_{c}<\dot{\gamma}$, the stress overshoots to $\sigma_{\text {plas }}$ before relaxing back to $\sigma_{s-s}$. Further, $\sigma_{\text {plas }}-\sigma_{s-s}$ increases as $\dot{\gamma}-\dot{\gamma}_{c}$ increases (the subscript s-s is used in this case because there is no pseudo-steady state, only a steady state.).

The corresponding values of these critical shear rates/stresses for the two concentrations presented here are displayed in table 5.1.

Table 5.1: Critical shear rates/stresses

\begin{tabular}{cccc}
\hline Concentration & $\dot{\gamma}_{\beta}\left(s^{-1}\right)$ & $\dot{\gamma}_{c}\left(s^{-1}\right)$ & $\sigma_{c}(P a)$ \\
\hline \hline $1.5 c^{*}$ & 0.005 & 0.050 & $8 \pm 0.25$ \\
$2.0 c^{*}$ & 0.001 & 0.035 & $11 \pm 0.25$ \\
\hline
\end{tabular}


The strain at which the pseudo-steady stress $\sigma_{p-s}$ starts increasing towards the common critical stress $\sigma_{c}$ is a function of the shear rate and scales as $\log (\gamma)$ as can be seen by the solid blue line in Fig. 5.4(b). This relation suggests a minimum strain of $\gamma=0.1$ is required for the stress to rise to the critical value. The rise in stress to $\sigma_{c}$ can be thought of as being a complex interplay between ageing and shear effects. In all cases where $\dot{\gamma}>\dot{\gamma}_{\beta}$, the duration of the stress overshoot is a linear function of time and hence also of strain.

The common critical stress for all rates $\dot{\gamma}<\dot{\gamma}_{c}$ at long times manifests itself as a plateau in the flow curve at $\sigma=\sigma_{c}$. The plateau extends from $\dot{\gamma}=\dot{\gamma}_{c}$, corresponding to the rate at which $\sigma_{p-s}=\sigma_{c}$, down to $\dot{\gamma} \sim 10^{-4} s^{-1}$. Such plateaux have been suggested as originating from an underlying constitutive flow curve that contains a mechanically unstable region of negative slope. In this case, that underlying constitutive curve, if it exists, is itself evolving from a mechanically stable monotonic curve. At long times, when the plateau is fully formed and the shear rate is kept constant at a value $10^{-6} s^{-1}<\dot{\gamma}<\dot{\gamma}_{c}$ spatially heterogeneous flow is expected.

We compare these results to those of a similar system of stars suspended in an intermediate solvent, decane, at $c=1.2 c^{*}$ [31]. In [31], Holmes et al. found significant hysteresis (thixotropy) in the flow curve and interpreted this as evidence for dynamic jamming/unjamming processes. There was found to be a time dependence between the length of time a shear rate was held and the shear stress achieved by the system that was thought to be due to sub-critical strain rates being applied. The results of the current work cannot discount this idea but through the use of the defined preshearing protocol, which has allowed accurate measurement of the sample's age, can offer further explanation. The thixotropy observed previously can be accounted for by the complex nature of the interplay between shear effects and ageing. In the language of the trap models $[84,85,86,87]$, there appears to be a balance found between the energy given to the system via shearing and the depth of the deepest traps. We note that this language may lead to a confusion between the shear-induced kinetics of the system and the role that energy plays in ageing the system. The authors would like the reader to think of this as merely a description in terms of the language of the trap models. At low shear rates, there is a small amount of energy given to the system such that traps of medium depth are able to trap an 'element'. As the shear rate increases, the energy given to the system also increases as well as the depth of the trap required to trap an element. As there are fewer deep traps, more time is needed for the system to fully 
explore the free energy landscape to find the traps capable of trapping the high energy 'elements'. This is reflected in the results of the transient start-up tests shown in Figs. 5.5 and 5.4 which show that as shear rate is increased (provided $\dot{\gamma}<\dot{\gamma}_{c}$ ), steady state, that is the time required for the stress to reach the plateau value, is achieved at longer times. As the shear rate is increased further, eventually enough energy is given to the system such that there are no traps deep enough to trap 'elements' and all the material flows for all time.

\subsubsection{Creep}

Complementary to the strain-rate controlled experiments of the previous subsection are stress-controlled experiments commonly referred to as creep experiments (see section 2.2). In a creep experiment the material is stressed and the subsequent acquisition of strain is measured. From this data the rate of strain accumulation can be calculated. This, in effect, allows an examination of the evolution of the flow curve with the stress axis as the abscissa and the rate axis as the ordinate. The fluidisation protocol remains unchanged. A stress of $8 \mathrm{~Pa}$ is applied to the $c=2 c^{*}$ material following spontaneous evolution for a time $t_{w}$ following fluidisation. The applied stress is specifically selected to be lower than the critical stress seen in the flow curves and shear start-up experiments in order to observe the evolution of the lower branch of the flow curve.

The results illustrated in Fig. 5.10 indicate that for $t^{\prime} \leq 200 \mathrm{~s}$ following waiting times $t_{w}<5000 \mathrm{~s}$ the material eventually acquires strain at a rate identical to that indicated by the short-time flow curve displayed as the yellow points in Fig. 5.8. For $t^{\prime} \leq 200 \mathrm{~s}$ following waiting times $t_{w} \geq 5000 \mathrm{~s}$ the accumulation of strain is orders of magnitude slower with a minimum accumulation rate of $10^{-5} \mathrm{~s}^{-1}$ after waiting $t_{w}=10000 \mathrm{~s}$ after fluidisation, suggesting the position of the lower branch of the flow curve.

The proportion of acquired strain recovered following the cessation of an $8 \mathrm{~Pa}$ stress is displayed in Fig. 5.11(a) for various waiting times. An interesting pattern is observed providing some insight into the evolution of the viscoelastic nature of the material. For waiting times $t_{w} \leq 1000 \mathrm{~s}$ the material recovers less than $4 \%$ of the strain acquired while under stress with the older sample recovering slightly more than the younger. When the waiting time is increased to $5000 \mathrm{~s}$, the material recovers nearly $20 \%$ of the strain acquired under stressing, indicating an evolution to a more elastic state. If allowed to evolve for $10000 s$ spontaneously, the amount of strain recovered $3000 s$ after 


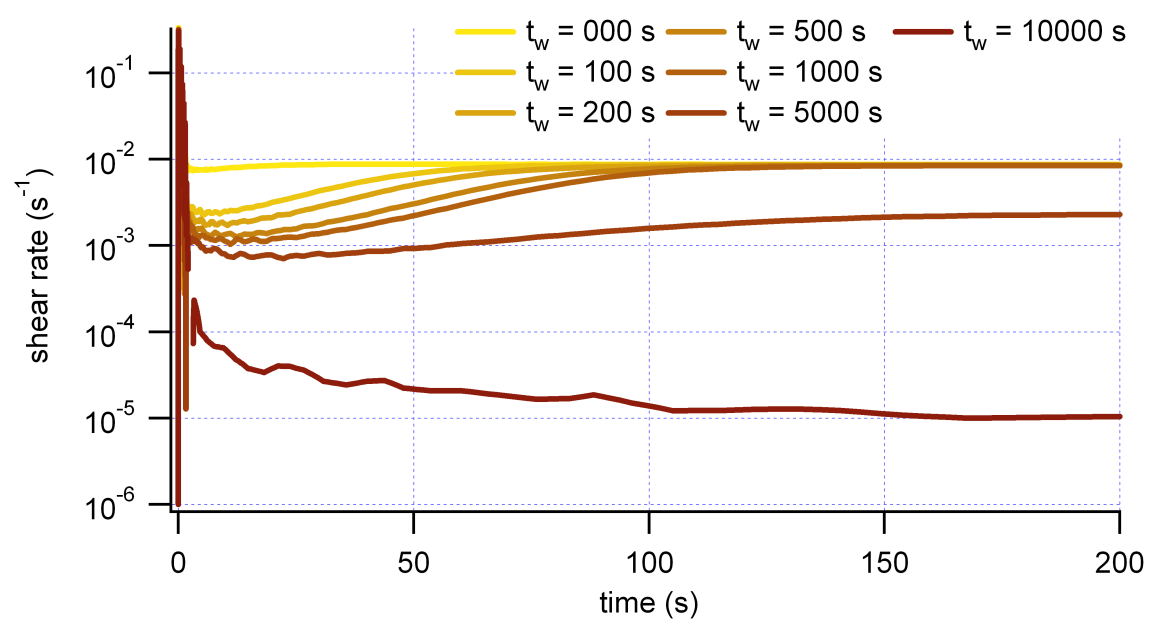

Figure 5.10: Rate of strain accumulation upon stressing the material at a constant $8 \mathrm{~Pa}$ after a waiting time $t_{w}$ indicated. For waiting times $t_{w}<5000 s$ the material is accumulating strain at the rate given by the early response of the flow curve (Fig. 5.8). For waiting times $t_{w} \geq 5000 s$ the material accumulates strain orders of magnitude slower than for shorter waiting times, such that for a waiting time of $t_{w}=10000 \mathrm{~s}$ the material accumulates strain at a rate of $10^{-5} \mathrm{~s}^{-1}$. This result gives an indication of the position of the lower branch of the flow curve at long times for stresses below the critical stress.

stress cessation is $97 \%$ of what is acquired under stress, indicating an almost perfectly elastic material. The material thus evolves from a nearly ideal liquid to a nearly ideal solid over the course of $10000 \mathrm{~s}$. Because the material does creep, and because there is not a perfect recovery of strain, the material still has a viscous nature, albeit significantly weaker than the elastic nature at an age greater than $10000 \mathrm{~s}$. Figure 5.11(b) shows a closeup of the strain recovery for a time $5 s$ after cessation of stress, highlighting the ringing nature at early times in the strain recovery. The amplitude of the exponential decay window of the ringing is approximately logarithmic. For a waiting time of $t_{w}=100 \mathrm{~s}$, the ringing has a maximum amplitude of approximately $0.5 \%$ of the acquired strain. For a waiting time $t_{w}=10000 \mathrm{~s}$ the ringing has a maximum amplitude of approximately $110 \%$ of the acquired strain; that is, the material momentarily recovers more strain than it had previously acquired. 

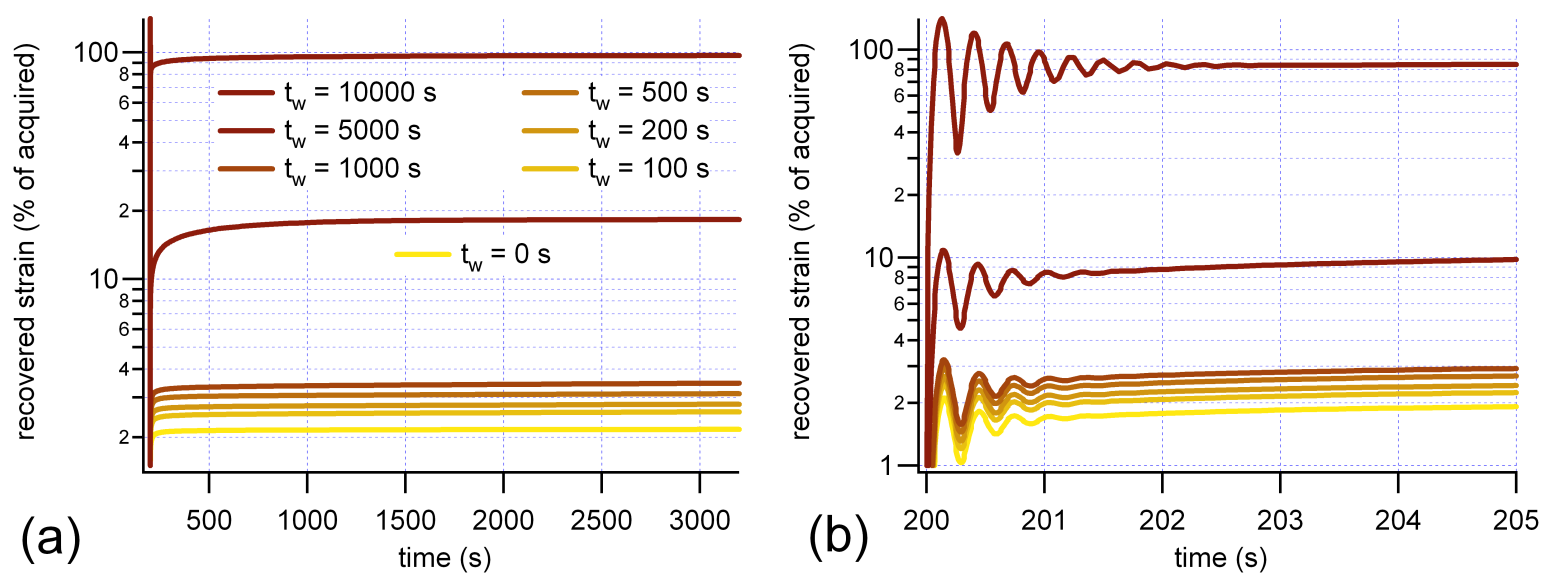

Figure 5.11: The percentage of acquired strain recovered following cessation after $200 \mathrm{~s}$ of an $8 P a$ stress as a function of time for different waiting times, $t_{w}$. (a) The long-time behaviour. (b) The first five seconds after stress cessation clearly showing ringing characteristic of elastic materials. The magnitude of the ringing increases with $t_{w}$.

\subsection{Stress Relaxation}

Having elucidated the form of the flow curve, we now report the effects of ageing on the $c=2 c^{*}$ sample. An identical protocol to that previously mentioned is employed to fluidise the sample, placing it in a history-independent state. After the fluidisation, the sample is left to evolve spontaneously for a time $t_{w}$ before a strain of $1 \%$, chosen so as to be in the linear regime, is imposed. The stress $\sigma(t)$ is recorded as a function of time and the results are displayed in Fig. 5.12. The relaxation of the stress depends on the waiting time, $t_{w}$ in a complex way. For values of $t_{w}$ up to $500 \mathrm{~s}$, the stress progressively takes longer to relax. For times $500 s \leq t_{w} \leq 3000 s$ the stress relaxes in an almost identical fashion as seen by the overlapping nature of the corresponding lines in Fig. 5.12. This overlapping for values of $t_{w}$ in the range $500-3000 \mathrm{~s}$ precludes any temporal rescaling such as used by Derec et al. in [47] that would allow a universal relaxation curve to be found. When $t_{w}$ is on the order of the time taken to reach the plateau in the flow curve, the stress no longer relaxes in a smooth, continuous manner but rather by a series of jumps which punctuate an otherwise very slow relaxation. That the stress relaxes completely, even for the longest $t_{w}$ measured suggests that the material does not form a glass, at least in the traditional sense. Instead the material behaves as a glass for times on the order of $500 \mathrm{~s}$, retaining internal stresses before reorganizing and 


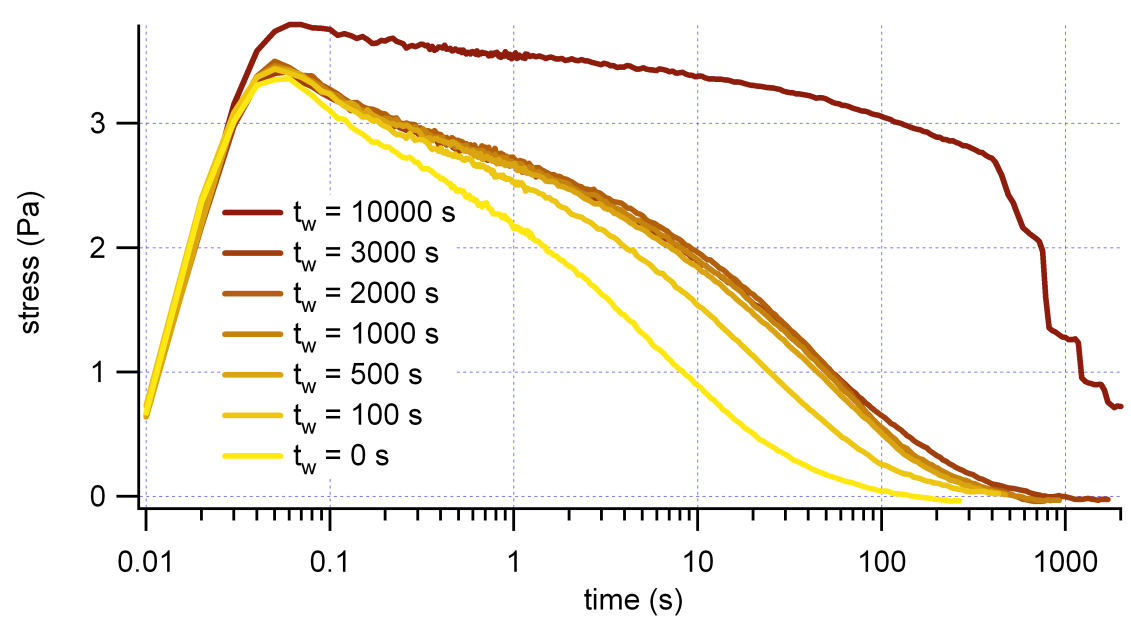

Figure 5.12: Stress relaxation as a function of time as response to a $1 \%$ strain following pre-shearing and varied waiting time $t_{w}$. Waiting times are indicated.

relaxing. Thus we have a 'short-lived' glass.

It is worth noting that the data displayed in Figs. 5.10 and 5.12 lead to the same conclusion: the evolution of the structure responsible for causing the plateau in the flow curves is not a result of shearing. Rather, it is a process that shearing disturbs in complex ways by way of adding more energy to the system via kinetics.

The effects of ageing on the stress relaxation after a step strain in the linear regime for the $c=2 c^{*}$ case are not able to be temporally rescaled to fit a single master curve. The slow relaxation of the stress when $t_{w}=10000 \mathrm{~s}$ as compared to when $t_{w}=0^{*} \mathrm{~s}$ indicates a transition to a material where stresses are nearly conserved upon application of a strain, i.e. a weak solid. We speculate that over the course of the waiting time $t_{w}$, the stars agglomerate into clusters. When a strain is applied, the clusters initially respond solidly. The clusters will then slowly relax the stored stress via retraction of the individual arms. Many retraction events later, an individual star, or group of stars, on the periphery may no longer be able to remain bound to the cluster and separates, relaxing large amounts of stress in a short amount of time. This speculative description offers a possible explanation for the slow relaxation punctuated by a series of finite jumps for the stress relaxation after the longest waiting time tested. 


\subsection{Ageing under oscillatory flow}

It has been shown in the previous sections that a suspension of star polymers in squalene undergoes a stiffening at times $t=O(10000 \mathrm{~s})$ after fluidisation when sheared continuously, where the specific time is a function of the applied shear rate. It has also been shown that when left to evolve spontaneously for a time $t_{w}$ after rejuvenation, the strain response to stresses below some critical stress changes from liquid-like for waiting times $t_{w} \leq 1000 \mathrm{~s}$ to solid-like for waiting times $t_{w}=10000 \mathrm{~s}$. When small strains are applied after a time of spontaneous evolution $t_{w}$, the material relaxes the developed stresses much slower and in a discrete, stepwise manner. In this final section of experimental results for this chapter, data are presented which examine the ageing response of the material via oscillatory strains.

Figure 5.13 is a plot of the evolution of the dynamic moduli when strained at an angular frequency of $\omega=1 \mathrm{rads}^{-1}$ with an amplitude of $\gamma_{0}=0.1 \%$ immediately following fluidisation. From the data of Fig. 5.1, it can be noted that such straining yields a response in the linear regime; that is, such strains do not break cages. The response of the material yields values identical to those displayed in Fig. 5.1 for times $t^{\prime}=t \leq 4000 \mathrm{~s}$. Between $t^{\prime}=t=4000 \mathrm{~s}$ and $t^{\prime}=t=10000 \mathrm{~s}$ the material undergoes a transition to a stiffer state, characterised by an increase in the storage modulus, $G^{\prime}$ of $\sim 25 \%$ and an $\sim 80 \%$ reduction in the loss modulus, $G^{\prime \prime}$.

Figure 5.13 has an identical form to results published by Helgeson et al. [29] for a system of identical star polymers in squalene at a concentration of $c=2.1 c^{*}$ under oscillatory shear at an angular frequency of $\omega=10 \mathrm{rads}^{-1}$ with a strain amplitude $\gamma_{0}=1 \%$. Small angle neutron scattering experiments exploring the microstructure of star polymer dispersions [88] show no evidence of crystallisation. It is not, therefore, believed that the stiffening observed is associated with a phase transition.

The evolution of the frequency response of the dynamic moduli in the linear regime has been examined and is displayed in Fig. 5.14. An identical fluidisation process was used to place the material in the same initial state. Immediately following fluidisation, the frequency dependence of the dynamic moduli was found, beginning at high frequencies. The strain amplitude $\gamma_{0}=0.1 \%$ ensured a response that corresponds to deep in the linear regime, two orders of strain-magnitude below the point where the dynamic moduli cross. Such a sweeping of frequencies lasted for $1900 \mathrm{~s}$. Upon cessation of the frequency sweep, a time $t=1000 \mathrm{~s}$ was allowed to elapse in which 


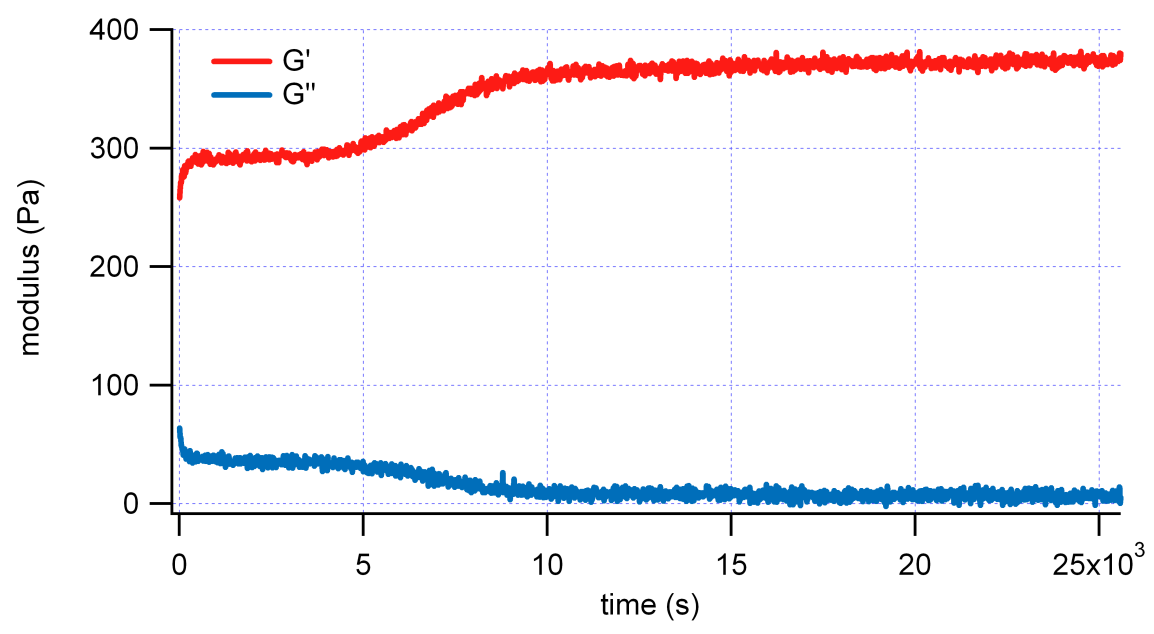

Figure 5.13: Evolution of the dynamic moduli under oscillatory shear of angular frequency $\omega=$ $1 \mathrm{rad} \mathrm{s}^{-1}$ with strain amplitude of $\gamma_{0}=0.1 \%$ showing the loss modulus (which is proportional to the energy dissipated per unit volume per cycle via equation 2.35) nearly disappear completely while the storage modulus increases by $\sim 25 \%$ indicating an evolution to a more solid-like response.

the material evolved spontaneously. Once $1000 \mathrm{~s}$ had elapsed since the end of the initial frequency sweep, a subsequent frequency sweep was carried out. This pattern of "frequency sweep - $1000 s$ - frequency sweep - $1000 s$ " was repeated many times. The results are depicted in Fig. 5.14 with power-law fits in the short- and long-time limits for both $G^{\prime}$ and $G^{\prime \prime}$. Low- and high-frequency limit power-law fits, with associated exponents are displayed for the loss modulus, $G^{\prime \prime}$.

Initially, the frequency response is identical to the data shown in Fig. 5.1(b), as expected. After the third frequency-sweep at a time $t^{\prime}=8700 \mathrm{~s}$ after fluidisation, a different response is obtained from the material that remains for the duration of the experiment: the storage modulus flattens over the frequencies applied so that $G^{\prime} \propto \omega^{0.028 \pm 0.003}$; at low frequencies the loss modulus decreases to approximately a third of the $t^{\prime}=0$ value and becomes noisier so that $G^{\prime \prime}\left(\omega \leq 0.5 \mathrm{rad} \mathrm{s}^{-1}\right) \propto \omega^{-0.45 \pm 0.2 \text {; }}$ at higher frequencies, the power-law exponent describing the loss modulus increases so that $G^{\prime \prime}\left(\omega \geq 7.5 \mathrm{rads}^{-1}\right) \propto \omega^{0.72 \pm 0.02}$. The broad minimum exhibited by the loss modulus of the youthful response of the material around $\sim 2 \mathrm{rads}^{-1}$ has lowered to $\sim 0.6 \mathrm{rads}^{-1}$, indicating an increase in size of particles being tested, suggesting the formation of clusters of stars. 


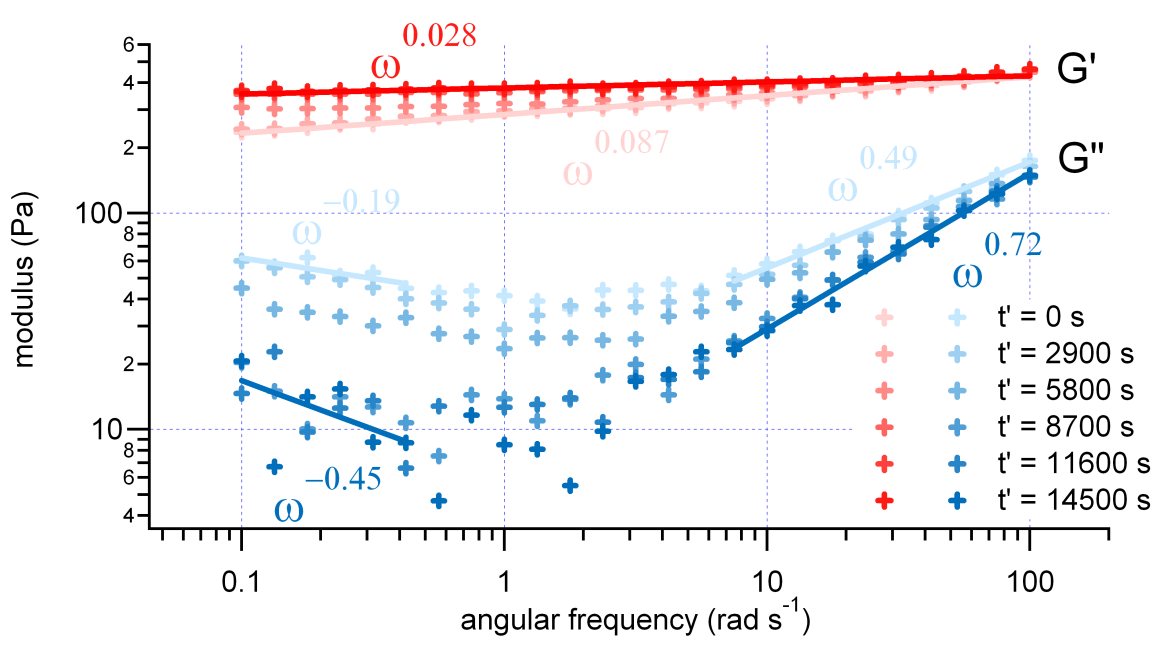

Figure 5.14: Evolution of the frequency response of the dynamic moduli with a strain amplitude of $0.1 \%$. Solid lines correspond to initial and final power-law fits.

\subsection{Conclusions}

The star polymers studied in the current work form a short-lived glass which, because of the stars' ability to interpenetrate, can be called a soft glass. The effects of the complex nature of the softness can be seen in the two-step stress evolution at shear rates below $\gamma_{c}$. This is not a phenomenon seen in hard glasses. There is an equivalence between the behaviour of the stars presented in this work and attraction-dominated colloidal glasses [89]. The interpenetration of the stars at concentrations above $c=c^{*}$ can be thought of as an effective attraction that must be overcome along with the breaking of the local cage in order for flow to occur. It is speculated that the two-step stress evolution is due to this dual-mode behaviour. It may be that at short times the stress response is dominated by arm-disengagement while at longer times the response is a combination of disengagements and the breaking of cages.

An oscillatory fluidisation protocol has been established and implemented in the investigation of the stress evolution and ageing of a colloidal glass made of star polymers of nominal functionality $f=128$ at two concentrations above the overlap concentration suspended in squalene at $293 \mathrm{~K}$. The protocol has allowed the elucidation of a complex dual-mode stress evolution under strain rate controlled conditions, below critical a value, $\dot{\gamma}_{c}$. This dual-mode evolution is seen in more general terms as a shear thinning, monotonic flow curve at early times after fluidisation which evolves into a 
flow curve dominated by a plateau at times on the order of $10^{4} \mathrm{~s}$ after fluidisation. The time taken for the system to reach the plateau stress increases as the applied shear rate approaches the critical value. It can thus be concluded that the stress plateau, and the corresponding steady increase in viscosity towards lower shear rates in the flow curve is a result of the interplay and eventual balance between ageing and shear effects. When sheared at rates above the critical value, i.e. for $\dot{\gamma}>\dot{\gamma}_{c}$ the system behaves as a shear-thinning fluid for all times.

Under stress-controlled conditions, it has been shown that the $c=2 c^{*}$ material evolves from a nearly ideal liquid to a nearly ideal solid over the course of $10000 \mathrm{~s}$, provided the stress applied is below the critical stress. Creep experiments have shown that for waiting times $t_{w} \leq 1000 \mathrm{~s}$, the material retains a nearly ideal-fluid response to a stress $\sigma<\sigma_{c}$. If allowed to evolve spontaneously for a time $t_{w}=10000 \mathrm{~s}$, the material recovers $97 \%$ of the strain acquired while under stress indicating a nearly ideal elastic solid-like response.

The fluidisation protocol has also allowed the study of ageing in the absence of shear via $t_{w}$-varied stress relaxation tests. No simple temporal rescaling exists which could be used to find a universal curve onto which all stress relaxations would fall. This can be seen by the overlap of stress relaxation for waiting times $t_{w}=500-3000 \mathrm{~s}$ and by the dramatically different mode of relaxation for $t_{w}=10^{4} \mathrm{~s}$. The stress caused by a sudden imposition of a strain of $1 \%$ relaxes even for the longest waiting time investigated $\left(t_{w}=10000 \mathrm{~s}\right)$, indicating the solid formed is not a glass in the traditional sense, but rather a short-lived glass that is able to relax stresses via rearrangements. It is postulated that over the course of a strain less period of time equal to $\sim 10000 \mathrm{~s}$ that individual stars coalesce into clusters that when strained break up in discrete events in order to relax stress. Under shearing, it is thought that the stress caused by the shearing acts as a growth inhibitor for clusters. Clusters that are limited in size are also limited in the amount of extra stress they can store. The idea of strain rate-limited cluster sizes offers an explanation for the evolution of the stress plateau in the flow curve. In the absence of shear, the uninhibited growth of clusters offers an explanation for the results seen in stress relaxation experiments and creep experiments where the applied stress is below that required to break the clusters apart.

The transition to a stiffer state has also been seen under linear-regime oscillatory flow via changes in the dynamic moduli. Constant frequency - constant amplitude shearing results in a two-step evolution of the storage and loss moduli similar to the 
stress response to continuous steady shear. It is thought that the initial response is dominated by individual stars and the long-time response by clusters of stars whose ability to flow past one another is far less than that of individual stars. This is further supported by the frequency response as a function of age, which shows the characteristic size of the particles increases as the material ages. 



\section{Chapter 6}

\section{Experimental: Velocimetry}

\subsection{Introduction}

In the previous chapters we have established the existence of a change in the behaviour of the concentrated suspension of stars in squalene under specific shear conditions. When the material is sheared in a constant uni-directional manner, the material exhibits a stiffening at times that are dependent on the shear rate. This stiffening manifests itself in the flow curve as a change from a shear-thinning fluid over all shear rates, to a flow curve dominated by a horizontal plateau, strongly suggestive of an evolution to some spatial heterogeneity in the flow behaviour. When sheared in an oscillatory manner, the stress response of the material shows a characteristic decrease that can also be associated with a spatial heterogeneity. The conventional rheological techniques used up to this point produce results that are indicative of the average behaviour of the entire experimental sample and are unable to resolve any spatial information. In this chapter, we use the techniques of rheo-NMR velocimetry [90] and conventional NMR to add a spatial dimension to our investigation.

\subsection{Geometry}

The geometry used to gain spatial and temporal information was a concentric cylinder rheo-NMR Couette device pictured in Fig. 6.1. It consists of an NMR tube of outer diameter $15 \mathrm{~mm}$ held in the centre of another NMR tube of outer diameter $20 \mathrm{~mm}$. The outer tube has a wall thickness of $1 \mathrm{~mm}$ to give a gap width of $1.5 \mathrm{~mm}$. The sample 
fluid, shown in red in Fig. 6.1 is placed in the gap between the two tubes and tracer fluid, shown in blue, is placed inside the central tube to allow slip measurements to be made simultaneously. The outer tube is held stationary by the NMR coil assembly while the inner tube is turned by a stepper motor connected to the inner tube via a rigid mechanical shaft. The stepper motor is able to apply rotations in the frequency range $0.1-14 \mathrm{~Hz}$. Between the mechanical shaft and stepper motor is a gearbox that allows low shear rates to be applied. The gearbox utilised in the experiments presented below has a ratio of 1:500. This ratio allows gap-average shear rates, $\overline{\dot{\gamma}}$, in the crossover region of the flow curve shown in Fig. 5.8 to be imposed on the sample fluid and velocity measurements to be made. We note that while it is possible to experimentally fix the average shear rate over the gap by fixing the rotational velocity of the inner wall, it is not possible to fix, or even measure, the stress. The shear stress will depend inversely on radius squared, as per equation 2.47 and the range of stresses attained by the material for a given gap-average shear rate is fixed by the (time-dependent) flow curve.

\subsection{Signal detection}

In practice, signal is acquired from a thin slice across the sample. Velocities perpendicular to that plane are detected via a pulse program that is a combination of a PGSTE (see section 3.11.3 and Fig. 3.18) with an imaging sequence (the addition of k-gradients, see section 3.11.1). A timing diagram for the sequence is shown in Fig. 6.2. The first soft pulse works in conjunction with a gradient in the $x$-direction to selectively excite spins in a slice $1 \mathrm{~mm}$ across the entire geometry. The second soft pulse, applied simultaneously with a gradient in the z-direction, selectively excites spins in a $20 \mathrm{~mm}$ slice transverse to the first slice so that the signal comes from a small fraction of the total sample. This scheme is illustrated in Fig. 6.3. The signal acquired produces twodimensional data files with $1024 k$ points and $32 q$ points (see equation 3.49 in section 3.11.3). Fourier transformation in the $k$ direction yields one-dimensional spatial information with a resolution of approximately $20 \mu \mathrm{m}$ while Fourier transformation along $q$ results in velocity probability distributions known as propagators (see section 3.11.4 and Fig. 3.19). The peaks of the propagators for each pixel across the gap give the most probable velocity for a given spin sited in the region covered by the pixel.

Experiments were carried out with gradient durations of $\delta=20 \mathrm{~ms}$ and an obser- 

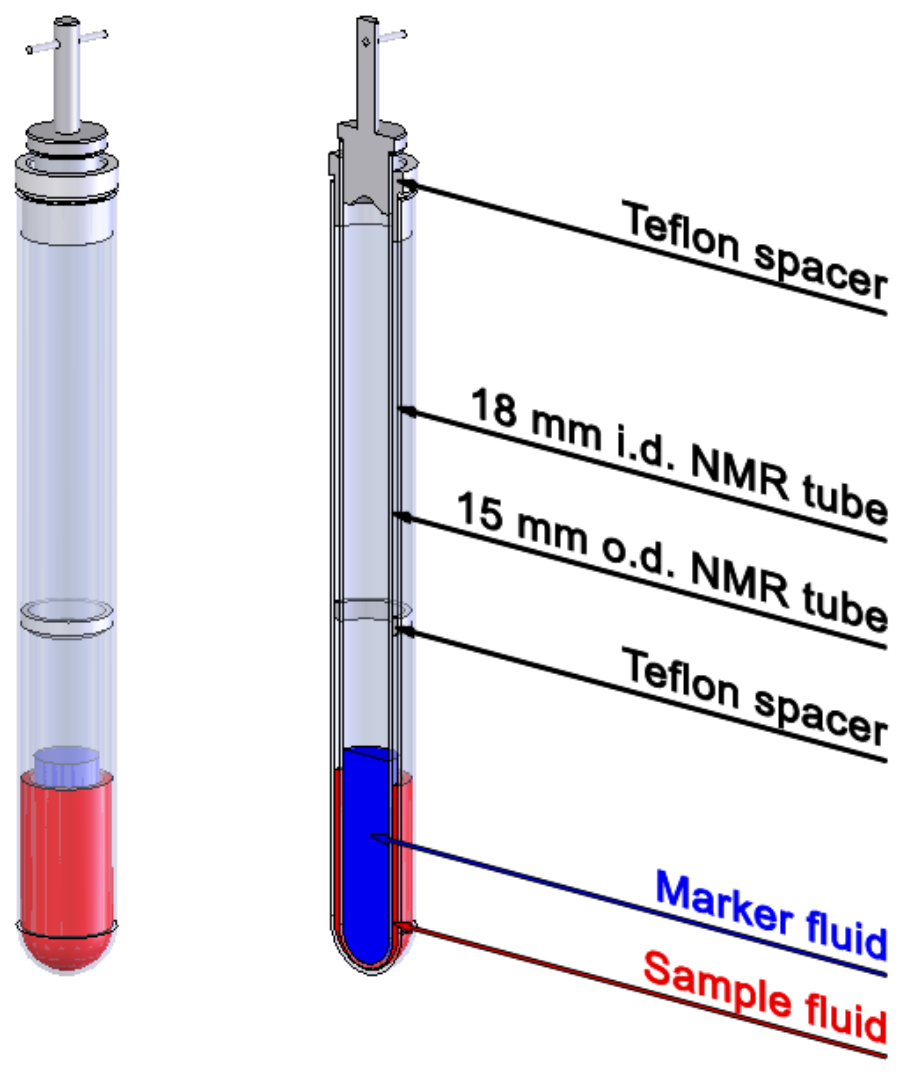

Figure 6.1: Representation of the experimental geometry. An NMR tube with outer diameter $15 \mathrm{~mm}$ is held coaxially inside another NMR tube of inner diameter $18 \mathrm{~mm}$ by way of two teflon spacers. A marker fluid, shown in blue, is placed in the inner tube and the sample to be tested, shown in red, is placed in the gap between the tubes. The outer tube is held stationary by the NMR coil, while the inner tube is rotated by a stepper motor. 


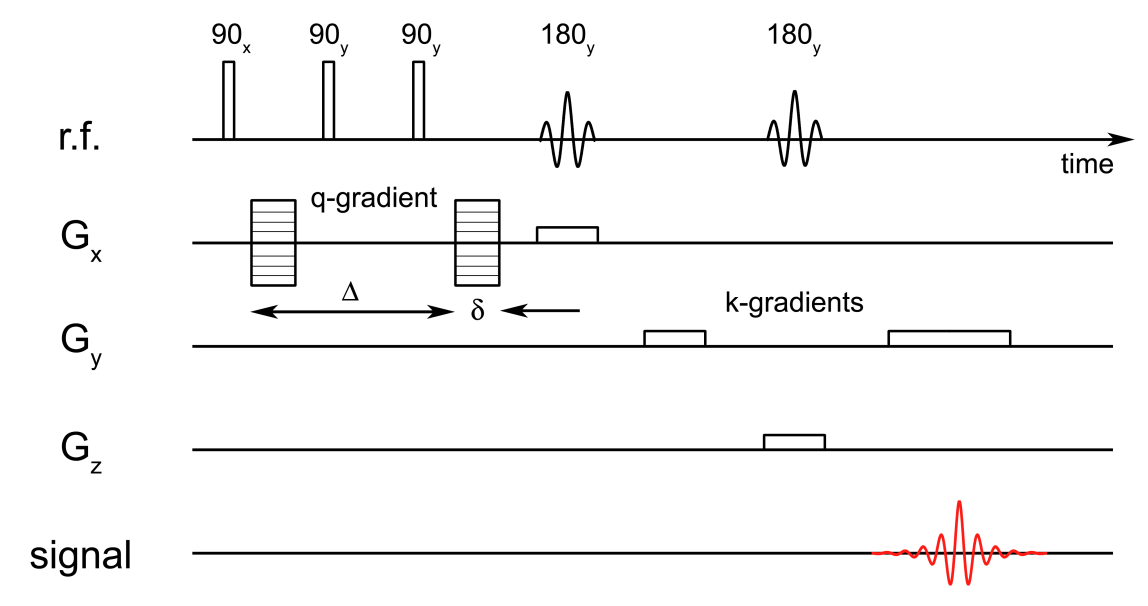

Figure 6.2: Timing diagram of the pulse sequence used to acquire velocimetry data. A combination of a PGSTE and an imaging sequence, the pulse sequence utilises dual slice selections to acquire onedimensional velocity profiles.

vation time of $\Delta=200 \mathrm{~ms}$. A total of eight scans were acquired in order to improve the signal-to-noise ratio. A single experiment lasts $150 \mathrm{~s}$ and makes up one 'frame' of a longer experiment when concatenated with successive frames. Typical larger experiments consist of 200 'frames' and last $30000 s$ or slightly shorter than $8 \frac{1}{2}$ hours.

\subsection{Experimental protocol}

The experimental protocol employed in the rheo-NMR experiments presented here is an altered version of that used in the traditional rheology measurements. At the time these experiments were carried out, smooth oscillatory shear had yet to be implemented in the rheo-NMR framework. As such, an oscillatory preshear was not possible and was replaced with a high-rate steady preshear. A gap-average shear rate of $0.767 \mathrm{~s}^{-1}$ was applied for $120 \mathrm{~s}$. The direction of the shear imposed during the experiment was the same as that imposed during the preshear. It was empirically determined that this preshear placed the material in a reproducible initial state.

The preshear was followed by a period of no shearing, referred to as the waiting time and denoted $t_{w}$, where the material is allowed to evolve spontaneously. The experimental time is denoted $t^{\prime}$ and has its origin at the end of the waiting period while the absolute time, simply denoted $t$, is set to zero at the end of the preshear. The abso- 

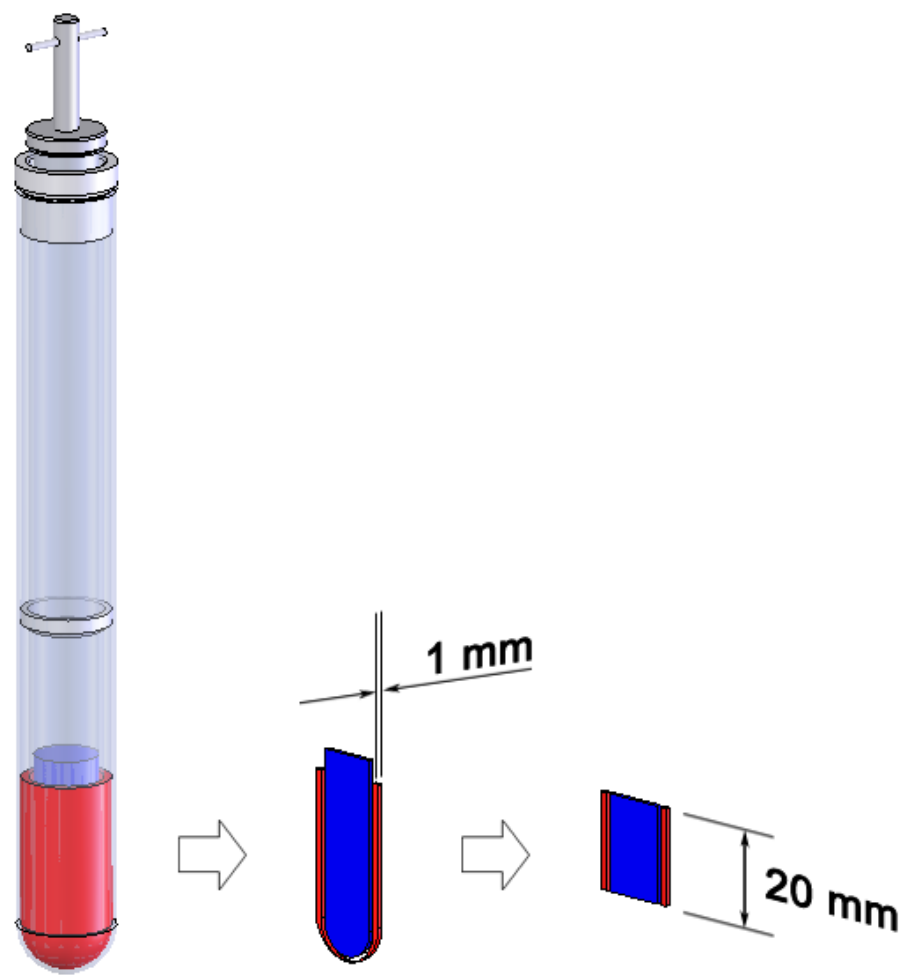

Figure 6.3: The signal is obtained from selective excitation of sample in a slice $1 \mathrm{~mm}$ wide and $20 \mathrm{~mm}$ thick. 
lute time, $t$, thus includes the waiting time while the experimental time, $t^{\prime}$ does not.

The temperature of the sample is held stable to within a tenth of a Kelvin by a temperature control unit that consists of a heating element working in combination with a constant gas flow. A thermocouple at the base of the Couette geometry records the temperature. A feedback loop determines the current through the heating element, allowing the temperature to be held within half a Kelvin of the set value. All samples were allowed to come into thermal equilibrium over a period of three hours before experimentation began.

\subsection{Evidence of shear banding}

Typical time-averaged one-dimensional velocity profiles with an imposed gap-average shear rate of $0.0314 \mathrm{~s}^{-1}$ at a temperature of $296 \mathrm{~K}$ with zero waiting time are displayed in Fig. 6.4. The imposed gap-average shear rate corresponds to a shear rate just below the critical value determined in the classical rheological experiments of chapter 5 . The data of Fig. 6.4 show the different responses of the material at two different times. The blue points in Fig. 6.4 (a) and (c) indicate the velocity of the unsheared tracer fluid (see Fig. 6.1) while the red points indicate the velocity of the sheared sample fluid. Figure 6.4 (b) and (d) show closeups of the velocity profile taken from one side of the geometry. Initially, Fig. 6.4 (a), the velocity profile indicates an entirely liquid-like response with no slip. After shearing at a constant shear rate for $30000 s$, the material is divided into two distinct shear bands as indicated by Fig. 6.4 (c) and (d). One band has a shear rate on the order of, but higher than, the gap-average shear rate of $0.0314 \mathrm{~s}^{-1}$, while the other band has a shear rate below the noise so that we may approximate it as being $0 s^{-1}$. The banded state is consistent with the existence of the plateau in the flow curve as shown in Fig. 5.8. While the plateau in the flow curve can only hint at the existence of some spatial heterogeneity, the methods of rheo-NMR allow a direct measurement of such shear banding.

\subsection{Evolution of banded structure}

The zero-shear band does not feature at all times in the evolution of the material response from preshear onwards. It is a spatial feature that has its inception at the outer 

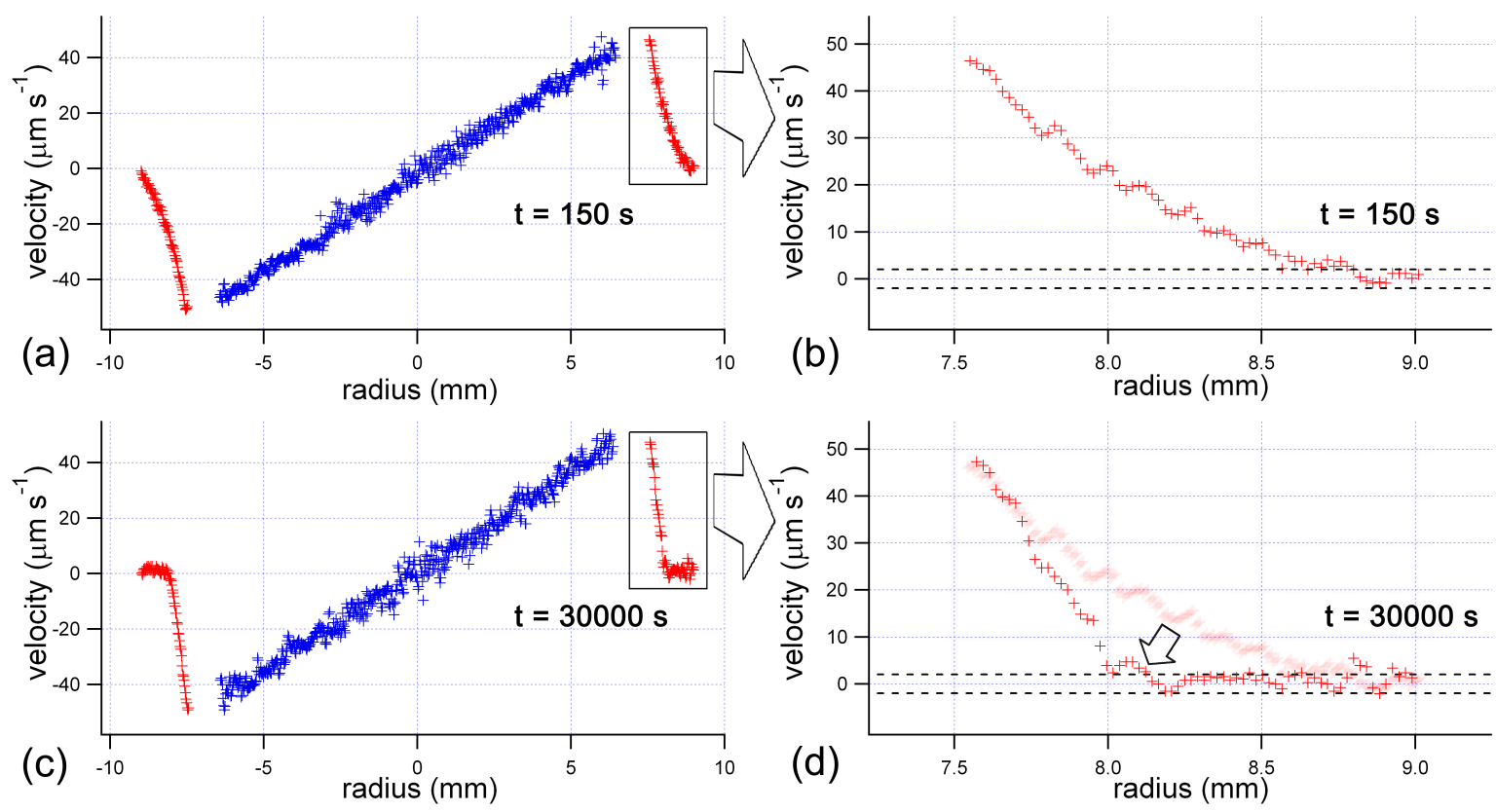

Figure 6.4: A one-dimensional velocity profile taken at times (a) $t=150 \mathrm{~s}$ and (c) $t=30000 \mathrm{~s}$ for an imposed gap-average shear rate of $0.0314 \mathrm{~s}^{-1}$ at a temperature of $296 \mathrm{~K}$ with a waiting time $t_{w}=0$. The purpose of the marker fluid is to allow for velocity extrapolation to determine the presence, or lack, of slip. No slip is seen during the entire experimental time-frame. (c) and (d) show close-ups of the velocity profile of the sample in the gap. A clear change can be seen from an initially liquid-everywhere response to a strongly banded state where one branch does not move. The dotted lines represent the limits used to track the evolution of the solid branch. 
edge of the Couette cell, where the stress is lowest. From this nascence at the stress minimum, the zero-shear band grows toward the inner wall, eventually reaching a stable limit. It is worth noting at this point that all velocity profiles taken in this study have a temporal resolution of $150 \mathrm{~s}$. Any temporal features whose periods are less than this limiting time are averaged out. Such time averaging is crucial for obtaining a reasonable signal-to-noise ratio from such little sample.

There are numerous methods available for calculating the relative proportion of gap displacement in the zero-shear band. The simplest may be to calculate the shear rate from the given velocity profile. This leads to problems, however, because of the noise inherent in the measurements. Numerical derivatives of noisy data tend to amplify the noise, making them hard to gather meaningful information from. The velocity data that comprise a single experiment consist of $\sim 200$ velocity profiles similar to those displayed in Fig. 6.4. Imperfections in the cylindricity of the inner tube of the Couette geometry on the order of a few microns will affect the spatial position of the velocity profile and also the velocities measured. To obtain a value for how much of the gap is in the zero-shear band we adopt the following approach. A lower limit on the resolvable velocities of $1.5 \mu \mathrm{m} \mathrm{s}^{-1}$ is set and the number of points across the gap whose velocity is lower than that limit is recorded. This limit is depicted by the dotted lines in Fig. 6.4 (c) and (d). The ratio of this number to the total number of points across the gap is calculated and this fraction is taken to represent the relative proportion of gap displacement that is in the zero-shear band.

There is an obvious limitation inherent in this method. There is always a non-zero proportion calculated, as shown by the data in Fig. 6.4 (b), even before the band has formed. This has greater consequences for calculating the proportional size of the zeroshear band at lower velocities, where a shallower profile is expected, but still allows some information to be gathered regarding size and growth rate.

A typical plot of the evolution of the relative proportion of gap displacement considered to be in the zero-shear band for an imposed gap-average shear rate of $0.0314 \mathrm{~s}^{-1}$ at a temperature of $296 \mathrm{~K}$ is shown as Fig. 6.5. The data of Fig. 6.5 correspond to tracking the extent of the gap displacement of the zero-shear band in the same experiment as the velocity profiles displayed in Fig. 6.4. It can be seen, from the data presented in Fig. 6.5, that up to approximately $4000 s$ the relative proportion of gap displacement of the zero-shear band is indiscernible from the noise. Therefore, the zero-shear band cannot conclusively be said to exist up to this time. After this initial period of latency, 


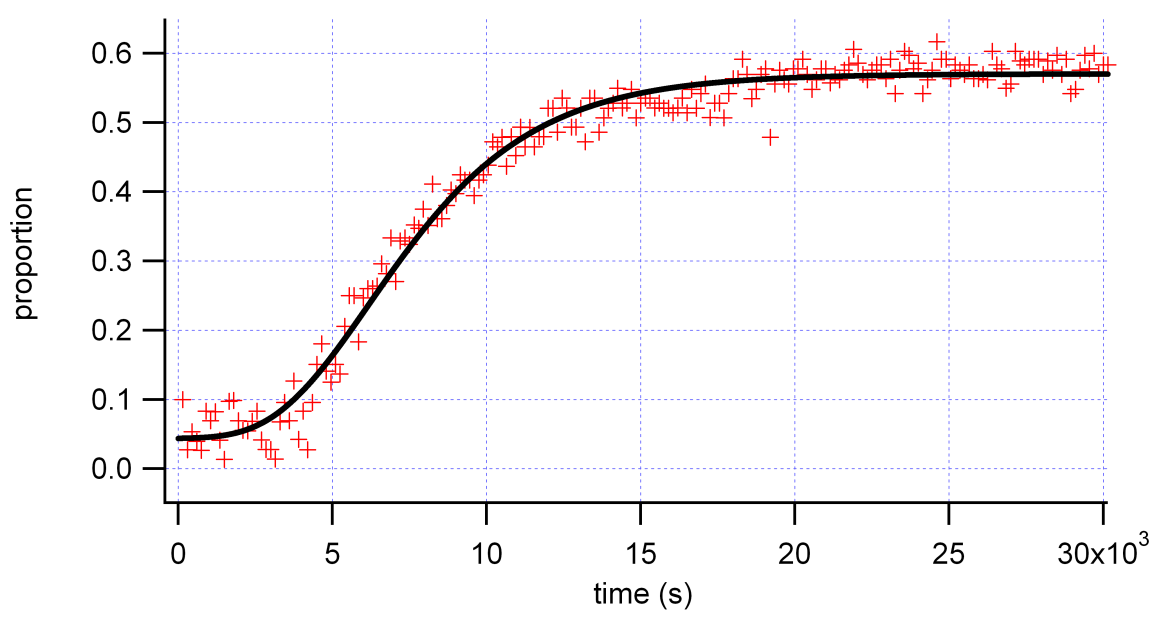

Figure 6.5: The evolution of the relative proportion of the gap displacement in the solid branch for an imposed gap-average shear rate of $0.0314 \mathrm{~s}^{-1}$ at a temperature of $296 \mathrm{~K}$ with a waiting time $t_{w}=0$ for a single experiment. The ordinates correspond to the proportion of the sample that has a velocity within the values represented by the dotted lines in Fig. 6.4. The solid line corresponds to a Gompertz function and describes the data well.

there exists a growth phase that lasts approximately $15000 \mathrm{~s}$. During this growth phase, the existing proportion monotonically approaches a final value. It will be shown that the final proportion of gap displacement in the zero-shear band is dependent on the imposed shear rate.

\subsection{Characterising the growth of the zero-shear band}

A growth relationship proposed by Gompertz [91] can phenomenologically be used to represent the growth of the zero-shear band. The Gompertz equation

$$
f(t)=f_{0}+\left(f_{\infty}-f_{0}\right) e^{-e^{-B(t-M)}}
$$

is a solution to the differential equation

$$
\frac{\mathrm{d} f(t)}{\mathrm{d} t}=B f(t) \ln \left(\frac{f_{\infty}}{f(t)}\right)
$$

where $f(t)$ is the relative proportion of the gap displacement in the zero-shear band, $f_{0}$ is the initial proportion (the lower asymptote), $f_{\infty}$ is the final proportion (the longtime limit), $B$ is the growth rate and $M$ is the time of maximum growth. The equation 
predicts growth at a rate proportional to the subject's size where the proportionality decreases as the size of the subject approaches an upper limit. The equation was initially proposed to account for age-dependent mortality rates in people [91], but has also been successfully used to describe mortality rates in many animals, see for example $[92,93,94]$ as well as growth of populations, organs and tumors [95, 96, 97, 98]. The equation has also been used to describe economic and social growth patterns as well as modelling the total number of papers in online scientific databases, for example [99, 100, 101].

The Gompertz growth equation has been used in the current study to represent the growth of the zero-shear band across the gap of a concentric-cylinder Couette geometry under various experimental conditions. The solid line in Fig. 6.5 is a Gompertz fit to the data, a fit that follows the evolution well. The following sections will focus on how the evolution of the zero-shear band changes, by way of changes in the fitting parameters when gap-average shear rate, waiting time, and temperature are altered.

\subsection{Experimental parameter effects on banding evolution}

\subsubsection{Imposed shear rate effects}

The data presented in Fig. 6.5 correspond to an imposed gap-average shear rate of $0.0314 \mathrm{~s}^{-1}$ at a temperature of $296 \mathrm{~K}$ with a waiting time of zero. The long-time limit as a function of imposed gap-average shear rate is displayed in Fig. 6.6 for three temperatures with no waiting time. The solid lines are linear fits. The range of imposed gap-average shear rates spans the critical rate of $0.035 \mathrm{~s}^{-1}$ determined by the classical rheological experiments of chapter 5 .

There are two conclusions to be drawn from the data of Fig. 6.6. One is that the total proportion of gap displacement in the zero-shear band decreases linearly, or very close to linearly, with increasing gap-average shear rate. The other is that an increase in temperature increases the proportion of the gap displacement in the zero-shear band.

The first of these conclusions can be understood in terms of the stress applied to the sample. While the exact stress is unknown and is not controllable in a controlled shear rate experiment, it is known that the stress varies across the gap in inverse squared proportion to the radius. This range of stresses means that when a gap-average shear rate is applied that correlates closely to the critical shear rate in the flow curve, Fig. 5.8, 


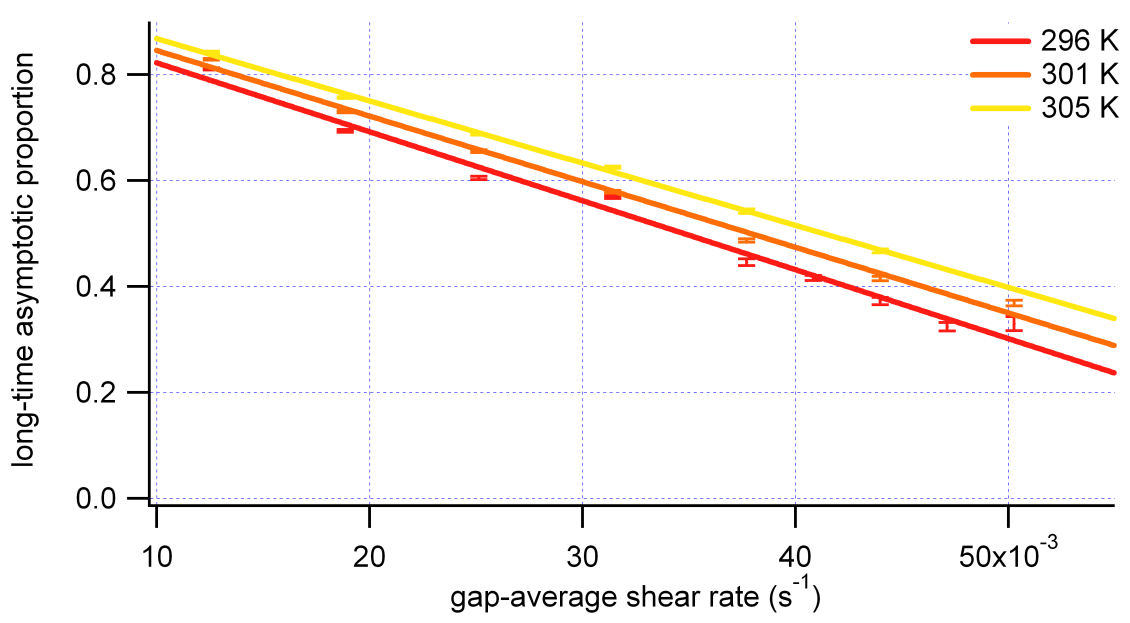

Figure 6.6: The long-time asymptotic proportion of relative gap displacement in the gap in the solid branch as a function of gap-average shear rate for three different temperatures with no waiting time between the end of the preshear and the beginning of the experimental shearing. Points correspond to Gompertz-fit parameters and their associated uncertainties and the lines are linear fits.

as the rates in Fig. 6.6 do, a portion of the gap will have a stress less then the critical stress, while the rest will have a stress above the critical stress. Fig. 6.6 thus allows a tracing of the critical stress across the gap of the Couette cell both as functions of imposed shear rate and temperature.

As a check, several experiments were performed where, once the relative proportion of gap displacement in the zero-shear band had plateaued, the imposed shear rate was changed. Typical results are displayed in Figs. 6.7 and 6.8, with fits, where the imposed gap-average shear rates were changed from $0.0471 \mathrm{~s}^{-1}$ and $0.0157 \mathrm{~s}^{-1}$, respectively to $0.0314 s^{-1}$. The fits were applied to each shear rate data set separately. The long-time limits of the relative proportion of gap displacement in the zero-shear band at each shear rate are identical to those found when a single shear rate is imposed on the sample.

The growth rate, B (see equation 6.1) as a function of imposed gap-average shear rate is displayed in Fig. 6.9 for three different temperatures. The waiting time for all the experiments whose fitting parameters are displayed in Fig. 6.9 is zero. Within the uncertainties $( \pm 5 \%)$, the power law exponents that can be used to describe the relationships between growth rate and gap-average shear rate are all -1 so that the growth rate may be described by a direct proportionality with $1 / \overline{\dot{\gamma}} \cdot 1 / \bar{\gamma}$ is the time taken for unit 


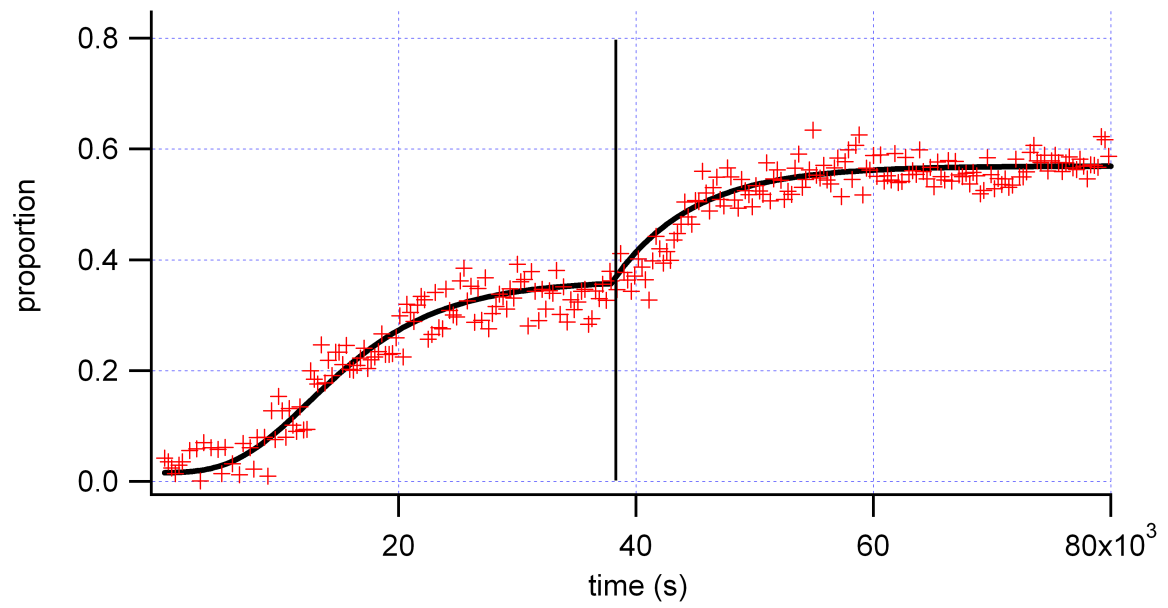

Figure 6.7: Relative proportion of gap displacement in the zero-shear band when the imposed gapaverage shear rate is decreased from $0.0471 \mathrm{~s}^{-1}$ to $0.0314 \mathrm{~s}^{-1}$ at a temperature of $296 \mathrm{~K}$ with a waiting time $t_{w}=0$. The solid lines are Gompertz fits and were applied to each shear rate data set separately. The vertical line indicates the time at which the imposed shear rate was changed.

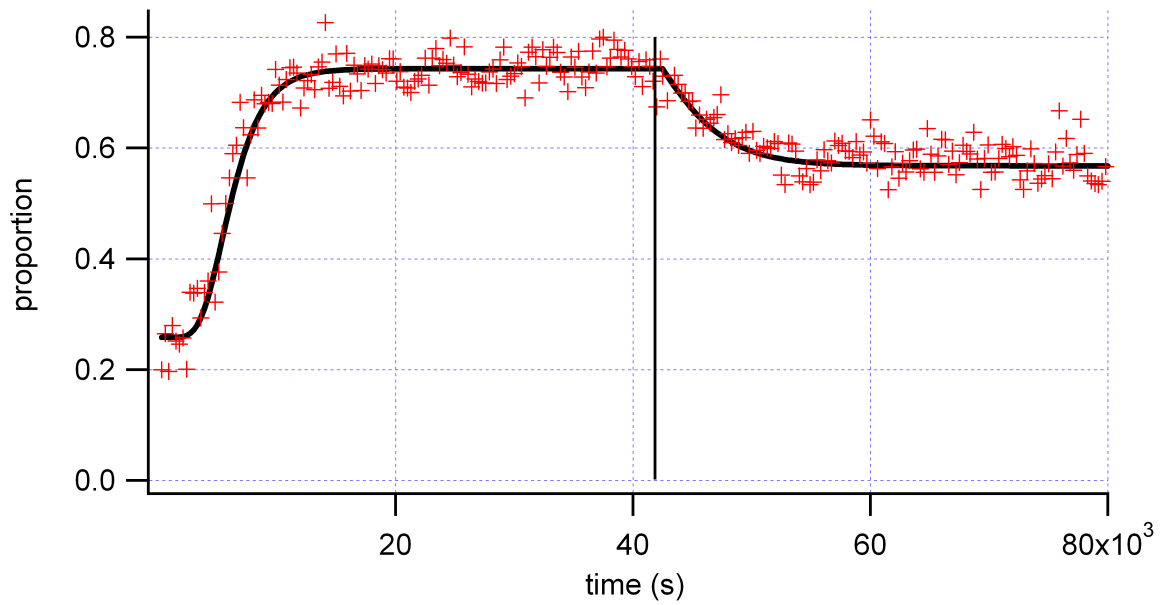

Figure 6.8: Relative proportion of gap displacement in the zero-shear band when the imposed gapaverage shear rate is increased from $0.0157 \mathrm{~s}^{-1}$ to $0.0314 \mathrm{~s}^{-1}$ at a temperature of $296 \mathrm{~K}$ with a waiting time $t_{w}=0$. The vertical line indicates the time at which the imposed shear rate was changed. The high initial proportion is due to the shallow nature of the velocity profile as discussed in section 6.6 of the text. 


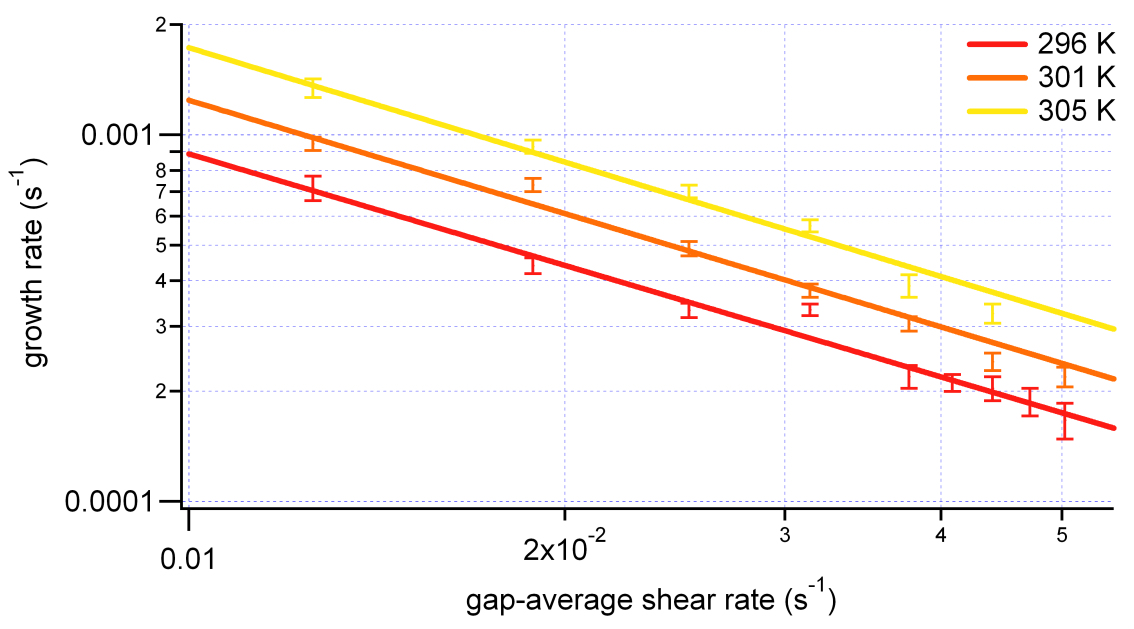

Figure 6.9: The growth rate of the proportion of sample in the solid band as a function of gap-average shear rate for three different temperatures with zero waiting time. Points correspond to Gompertz-fit parameters and their associated uncertainties and the lines are power-law fits.

strain to occur in the material and the characteristic time scale of the experiment.

The maximum growth time as a function of imposed gap-average shear rate for experiments with zero waiting time is displayed in Fig. 6.10 for three different temperatures. The maximum growth time shows an exponential dependence on the imposed gap-average shear rate with the exponential constant also being a function of temperature. The zero-shear band thus forms faster at higher temperatures and lower shear rates.

The data presented in Figs. 6.6 - 6.10 indicate that an increase in shear rate decreases the overall proportion of gap displacement in the zero-shear band. The growth of the zero-shear band is also slowed by shearing, with the time of greatest growth occurring later when higher shear rates are imposed.

\subsubsection{Waiting time effects}

Non-zero waiting times have the effect of allowing the material to relax stresses residual from the preshear over the course of $t_{w}$ and evolve spontaneously. The spontaneous evolution occurs in the absence of forced kinetics and involves predominantly thermodynamic processes, thus allowing insight into the interplay between the two. The effects of non-zero waiting times on the evolution of the zero-shear band have been in- 


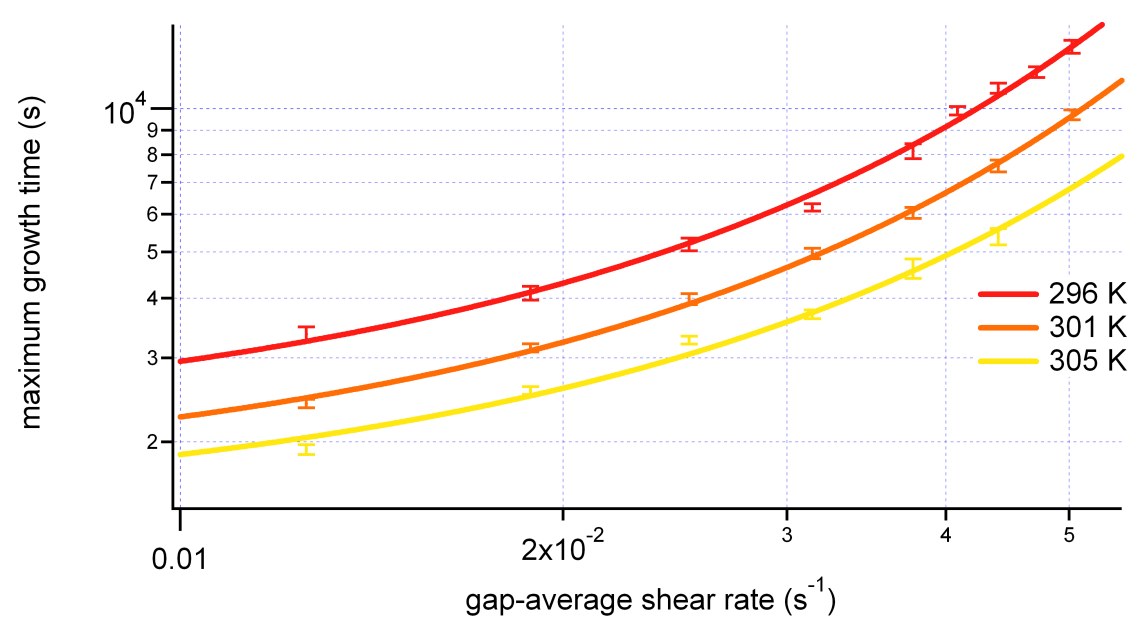

Figure 6.10: The time of maximum growth of the relative proportion of gap displacement in the solid band as a function of gap-average shear rate for three different temperatures with zero waiting time. Points correspond to Gompertz-fit parameters and their associated uncertainties and the lines are exponential fits.

vestigated. While varying the waiting time has no effect on the long-time asymptotic proportion, as would be expected, varying the waiting time does have an effect on the timing and rate at which the zero-shear band approaches the maximum extent.

The growth rate as a function of waiting time for a gap-average shear rate of $0.0314 \mathrm{~s}^{-1}$ is displayed in Fig. 6.11 for three different temperatures. The data exhibit a minimum around $100 \mathrm{~s}$. For waiting times $t_{w}<100 \mathrm{~s}$ the trend is weakly decreasing growth rates with increasing waiting time and the trend for waiting times $t_{w}>100 \mathrm{~s}$ is a powerlaw dependence of the growth rate on the waiting time. Within the uncertainties, all the power-law exponents are the same, a sub-linear value of $0.69 \pm 0.04$. From the data of Fig. 5.3, it can be gathered that over the course of $100 s$ of spontaneous evolution, the stress residual from the preshear drops to $\sim 15 \%$ of its initial value. The data of Fig. 6.11 can then possibly be interpreted as suggesting stresses residual from the preshear hinder the growth of the structure responsible for the zero-shear band. The zero-shear band thus forms as a competition between the (relaxing) stress residual from preshear and the underlying minimisation of energy in the presence of kinetic processes.

Figure 6.12 is a plot of the absolute time of maximum growth, $t_{M}^{\prime}+t_{w}$ as a function of waiting time for three different temperatures. There is a clear distinction to be made 


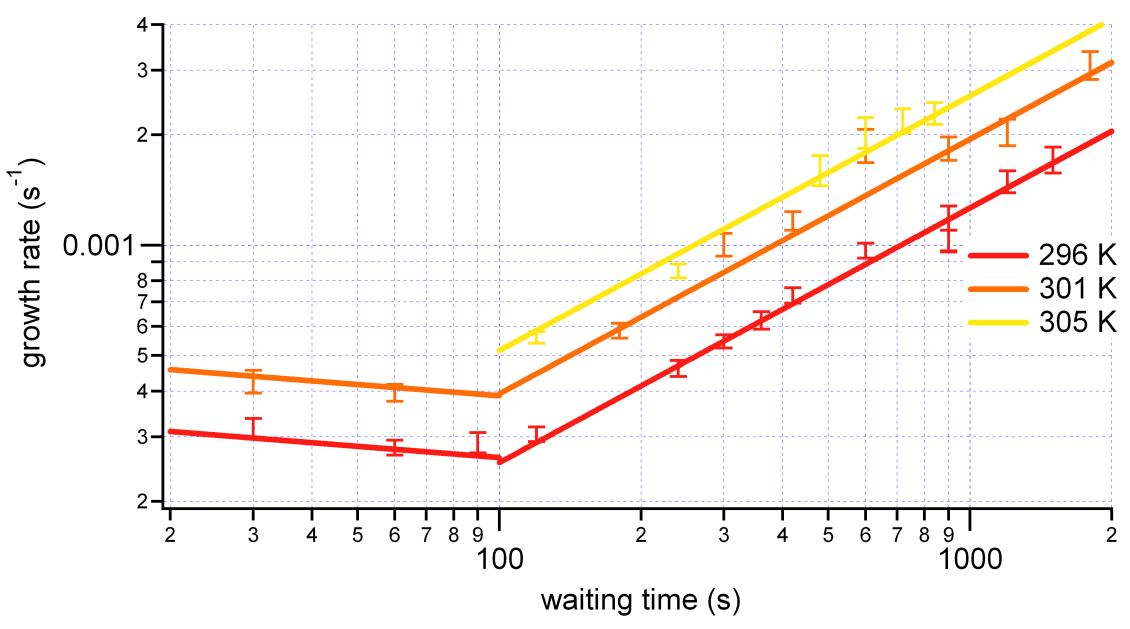

Figure 6.11: The growth rate of the relative proportion of gap displacement in the solid band as a function of waiting time for three different temperatures for an imposed gap-average shear rate of $0.0314 \mathrm{~s}^{-1}$. Points correspond to Gompertz-fit parameters and their associated uncertainties and the lines are power-law fits.

between the experimental time of maximum growth, which does not include the waiting time, and the absolute time of maximum growth, which does. It is clear from the data of Fig. 6.12 that the absolute time of maximum growth has two distinct regimes on either side of a critical value of the waiting time, $t_{w(c)}$. For waiting times $t_{w}<t_{w(c)}$, the absolute time at which maximum growth occurs is a decreasing linear function of the waiting time, while for waiting times $t_{w} \geq t_{w(c)}$, the maximum growth time is a constant. The time of maximum growth, for a given value of the waiting time, is shorter for higher temperatures. The critical waiting time, $t_{w(c)}$ is lower for higher temperatures. That is, for a greater input of thermal energy, maximal growth is achieved sooner.

The data of Figs. 6.11 and 6.12 suggest that non-zero waiting times have multiple effects on the evolution of the zero-shear band. The two-regime data of Fig. 6.11 suggest that a waiting time $t_{w}<\sim 100 s$ acts to allow stresses residual from the preshear, stresses that inhibit growth of the zero-shear band, to dissipate. This effect is manifested in the decrease of growth time for waiting times up to this limit. For waiting times $t_{w} \geq \sim 100 \mathrm{~s}$, any stress residual from the preshear has dissipated and the material is allowed to spontaneously evolve.

If the waiting time is greater than some temperature-dependent value, $t_{w}^{*}$, then the 


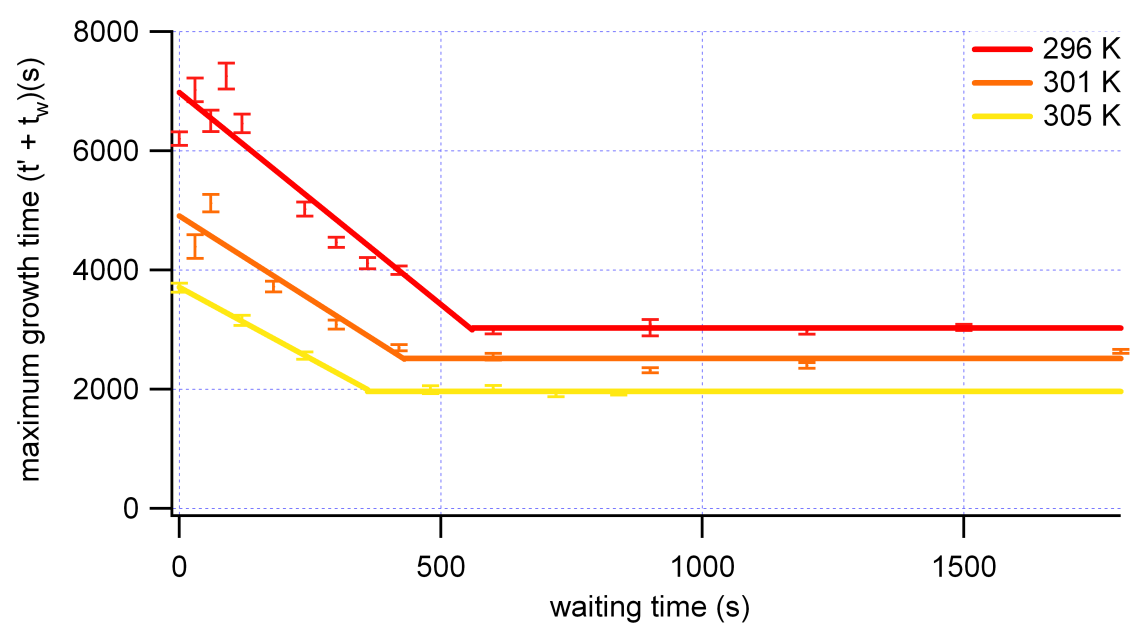

Figure 6.12: The time of maximum growth of the relative proportion of gap displacement in the solid band as a function of waiting time for three different temperatures for an imposed gap-average shear rate of $0.0314 \mathrm{~s}^{-1}$. Points correspond to Gompertz-fit parameters and their associated uncertainties and the lines are linear fits.

relative proportion of gap displacement in the zero-shear band will be greater than the noise at $t^{\prime}=0$. This is illustrated by the data of Fig. 6.13, where the evolution of the relative proportion of gap displacement in the zero-shear band is plotted as a function of experimental time, $t^{\prime}$, for two different values of the waiting time, $t_{w}=$ 0 (red) and $t_{w}=1800 \mathrm{~s}$ (yellow) at a temperature of $333 \mathrm{~K}$ and an imposed gap-average shear rate of $0.0314 \mathrm{~s}^{-1}$. The proportion of gap displacement in the zero-shear band at an experimental time $t^{\prime}=0$ after a waiting time of $t_{w}=1800 \mathrm{~s}$ (yellow points) is equal to the long-time limit of the zero waiting time example, indicating the zero-shear band is not a consequence of flow. Rather, flow allows us a method of probing the extent of the structure responsible for the zero-shear band. It is also apparent from the data of Fig. 6.9 that shearing slows the growth of the structure responsible for the zero-shear band. The data of Fig. 6.13 suggest that in the absence of shearing, all the sample in the gap would eventually become part of the structure responsible for what, under shear, becomes the zero-shear band. 


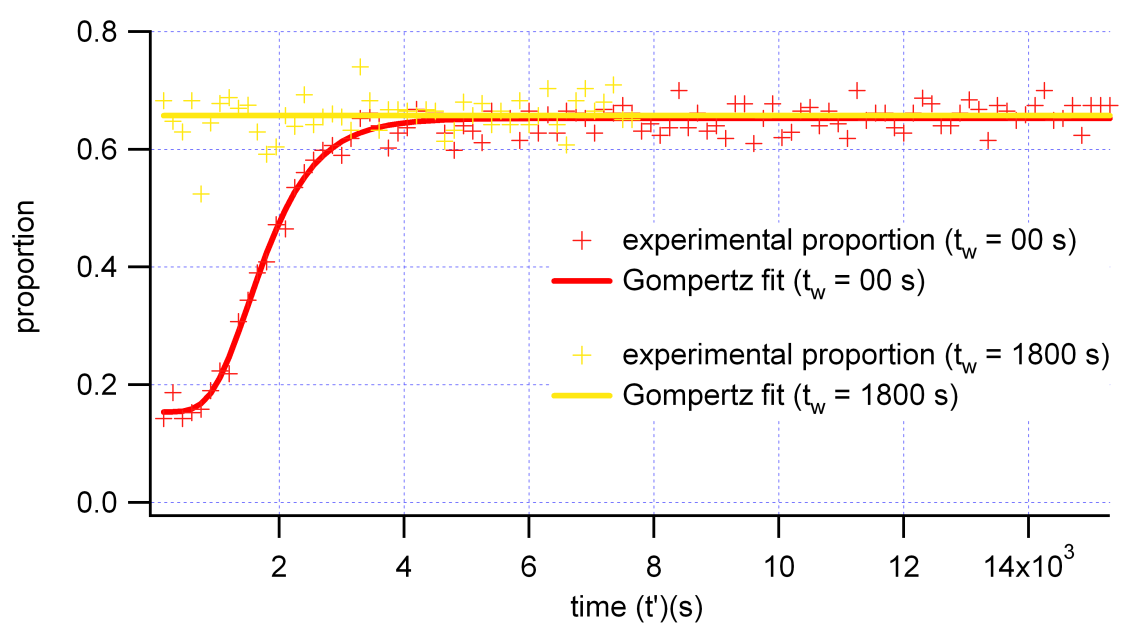

Figure 6.13: Evolution of the relative proportion of gap displacement in the zero-shear band at a temperature of $333 \mathrm{~K}$ for waiting times, $t_{w}=0 \mathrm{~s}$ (red) and $t_{w}=1800 \mathrm{~s}$ (yellow) for an imposed gapaverage shear rate of $0.0314 \mathrm{~s}^{-1}$ with their associated Gompertz fits (solid lines).

\subsubsection{Temperature effects}

The major emphasis of this chapter up to now has been on determining the effects on the Gompertzian fitting parameters of altering the imposed gap-average shear rate and waiting time. Figures 6.6 - 6.12 have focussed on these experimental parameters with a minor emphasis on temperature effects. In this last section, a closer look is taken into how altering the temperature effects the evolution of the zero-shear band when the applied gap-average shear rate is $0.0314 \mathrm{~s}^{-1}$ and there is zero waiting time. Kapnistos et al. [30] have shown the existence of a reversible thermal gelation in systems of identical stars in decane. The gelation is attributed to the swelling of the stars with an increase in temperature. Unpublished dynamic light scattering data from the same group show the stars have a nearly constant hydrodynamic radius of $50 \mathrm{~nm}$ in squalene over the temperature range $288-333 \mathrm{~K}$. Changes in temperature over this range will therefore only act to change the amount of thermal energy in the system and not the physical properties of the system itself.

The long-time limit as a function of temperature is shown in Fig. 6.14. It is clear from the data of Fig. 6.14 that the zero-shear band extends further across the gap at higher temperatures. The data of Fig. 6.14 can been described by an exponential as a function of shifted temperature, $T-T^{*}$, over the temperature range covered, with an 


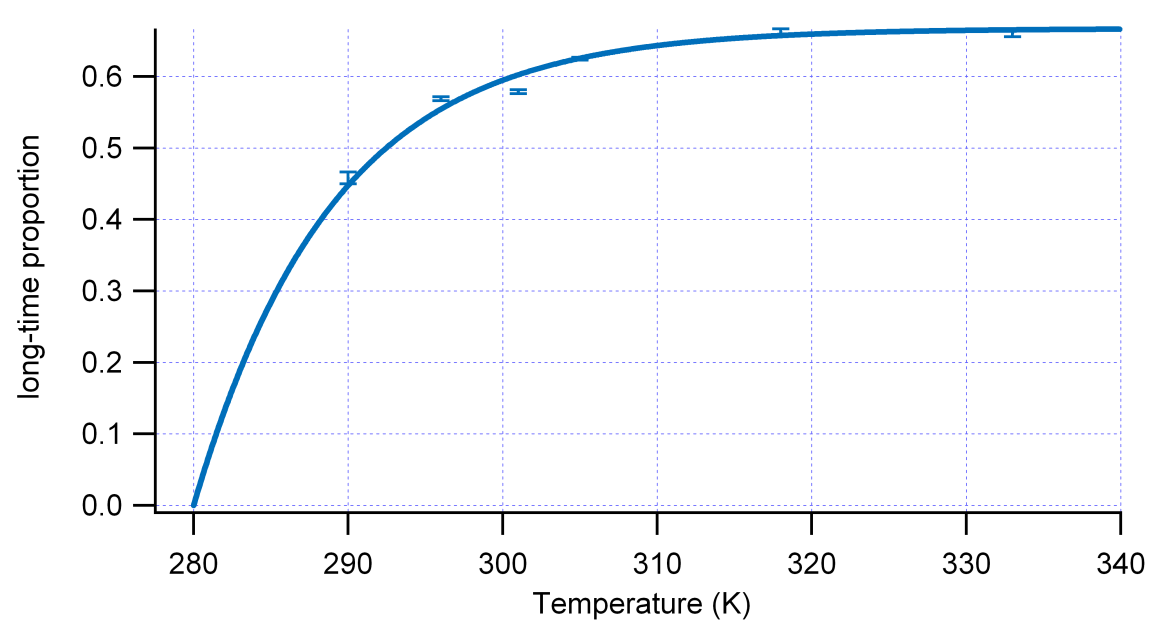

Figure 6.14: The upper asymptotic relative proportion of gap displacement in the solid branch as a function of temperature with an applied gap-average shear rate of $0.0314 \mathrm{~s}^{-1}$ and a waiting time $t_{w}=0$. Points correspond to Gompertz-fit parameters and their associated uncertainties and the solid line is an exponential fit.

upper limit of $0.67 \pm 0.01$ and a temperature-constant of $(10 \pm 2) K$. The temperature shift is found to be $278 \pm 2.5 \mathrm{~K}$. This dependence on shifted temperature suggests that for temperatures $T<T^{*}$ the zero-shear band does not form, although there is no data in this temperature range to support this. It is possible to interpret this result as the existence of a minimum amount of thermal energy required for entanglements that lead to kinetic arrest.

The natural logarithm of the growth rate is plotted as a function of inverse temperature in an Arrhenius manner in Fig. 6.15. The Arrhenius equation gives the rate constant of a chemical reaction, $k^{\prime}$ s dependence on the temperature, $T$, and activation energy, $E_{a}$ :

$$
k=A \exp \left(\frac{-E_{a}}{k_{B} T}\right)
$$

where $A$ is referred to as the prefactor and $k_{B}$ is the Boltzmann constant. If the data of Fig. 6.15 is interpreted as being Arrhenius-like, a plot of $\ln$ (growth rate, $B$ ) as a function of $T^{-1}$ ought to be in the form of a straight line with a y-intercept of $\ln (A)$ and a gradient of $-E_{a} / k_{B}$. A linear fit is applied to the data of Fig. 6.15 that yields a prefactor of $A=1426 \pm 180 \mathrm{~s}^{-1}$ and an activation temperature, $E_{a} / k_{B}$, of $4530 \pm 280 \mathrm{~K}$. From kinetic theory, the fraction of molecules that have a kinetic energy greater than $E_{a}$ in 


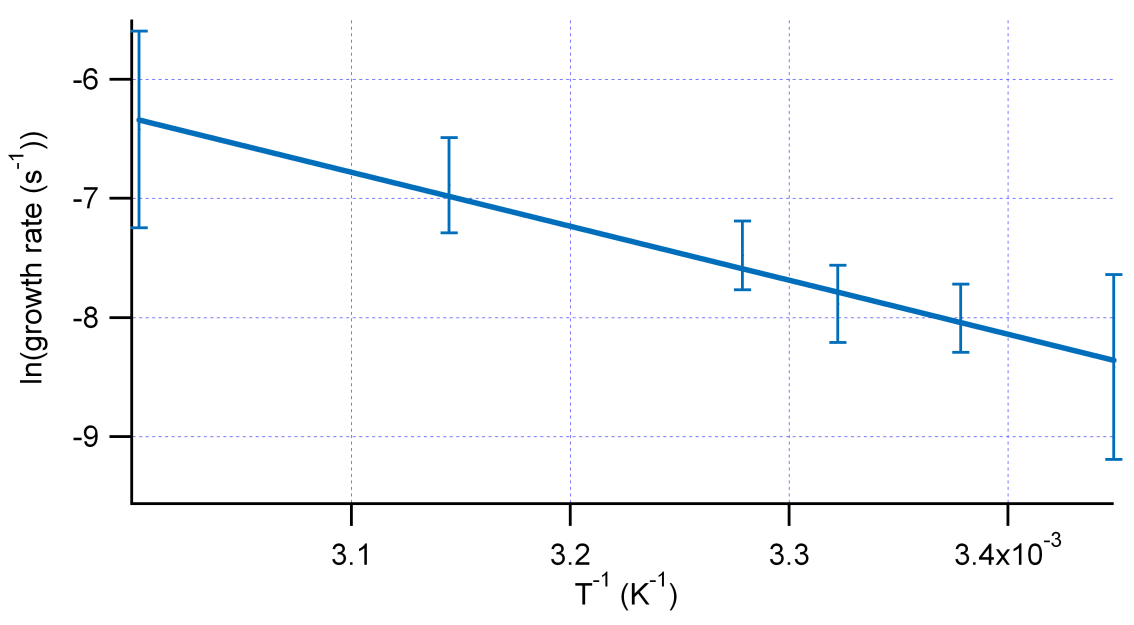

Figure 6.15: The logarithm of the growth rate of the relative proportion of gap displacement in the solid band as a function of inverse temperature with an applied gap-average shear rate of $0.0314 \mathrm{~s}^{-1}$ and a waiting time $t_{w}=0$. Points correspond to Gompertz-fit parameters and their associated uncertainties and the solid line is an Arrhenius fit.

a macroscopic sample can be calculated from the Maxwell-Boltzmann distribution of statistical mechanics, and is proportional to $e^{-E_{a} / k_{B} T}$. Treating the stars as perfectly elastic hard spheres thus yields a very small fraction of interacting stars responsible for clustering. At a temperature of $312 \mathrm{~K}$, the growth rate will be twice as fast as that when the temperature is $296 \mathrm{~K}$.

The temperature dependence of the maximum growth time is plotted in Fig. 6.16. The fundamental relation is that higher temperatures cause maximal growth sooner than lower temperatures. There is a linear relation between the time of maximum growth and inverse of the shifted temperature, $\left(T-T^{*}\right)^{-1}$ with a temperature shift of $T^{*}=281.5 \pm 0.5 \mathrm{~K}$. This temperature shift is equal, within the experimental uncertainties, to that found in the temperature dependence of the long-time limit and suggests the same physics.

\subsection{Regarding the Gompertz equation}

Before continuing further, it is worth making a note about a possible physical meaning of the Gompertz equation in the present context and the way it describes the data successfully. It is the main thesis of this work that the stiffening observed in conventional 


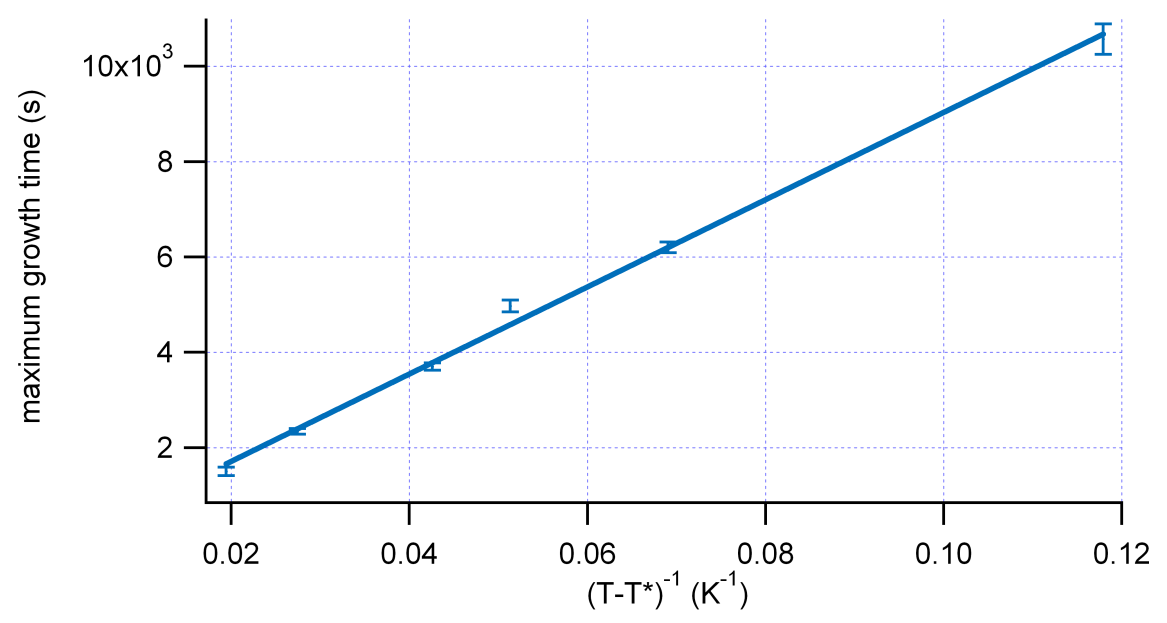

Figure 6.16: The time of maximum growth of the relative proportion of gap displacement in the solid band as a function of inverse shifted temperature, $\left(T-T^{*}\right)^{-1}$, with an applied gap-average shear rate of $0.0314 \mathrm{~s}^{-1}$ and zero waiting time. Points correspond to Gompertz-fit parameters and their associated uncertainties and the solid line is a linear fit. The shift temperature is $281.5 \pm 0.5 \mathrm{~K}$.

rheological experiments, the spatial heterogeneity suggested by LAOS measurements and the existence of the zero-shear band in NMR velocimetry experiments is due to the presence and growth of clusters of stars. The growth and extent of the zero-shear band has been seen in the previous sections to be limited by shearing. Rather than being shear-rate dependent, the data of chapter 5 suggest that the limiting factor is the proximity to some critical stress. We can thus interpret the Gompertz equation's exponential growth phase at early times as describing the initial enlargement of the clusters due only to their size. A smaller cluster has less surface area than a larger one, and so larger clusters initially grow faster. The initial exponential growth is curbed by the decreasing surface-to-volume ratio. Cluster size is limited by the extra stress caused by the presence of clusters, and so the growth slows and asymptotically approaches an upper limit set by the stress profile of the containing geometry.

\subsection{Structure of the 'zero-shear band'}

The results presented in the previous section make clear the existence of some agedependent structure that manifests itself as a zero-shear band in velocimetry experiments. It has been shown that under shear this structure is limited in size, with the 
extent of the structure being dependent on the applied gap-average shear rate. In the absence of shear, the structure is not limited in size and can grow to encompass the entire sample volume. Factors that control the growth of the structure include imposed shear rate, temperature and waiting time. The nature of this structure shall be the subject of this concluding section.

Recall the discussion of section 3.3.1 where it was shown that nuclei with spin $I \geq 1$ in a liquid exhibit a spectral splitting that is dependent on physical alignment. The physical alignment can be caused by the experimental apparatus or be flow related. Such results have been seen in the spectral analysis of a lamellar system doped with heavy water confined to a narrow-gap Couette geometry [102]. Unlike the work of Lutti et al. [102], where the lamellar system has a definite orientation that changes with flow, the arms of the stars investigated in this study are isotropically distributed about the central core. Nevertheless, it is worthwhile to test for alignment that may be present in the structure that manifests itself as the zero-shear band in the velocimetry experiments.

Per-deuterated stars were treated using an identical shear protocol to the protonated stars. Spectra were acquired immediately following preshear and after shearing at an imposed gap-average shear rate of $0.0314 \mathrm{~s}^{-1}$ for $36000 \mathrm{~s}$, a period of time long enough so that the banding had reached the steady state. The spectra are expected to be composed of two peaks with an intensity ratio of 2:1 corresponding to twice as much signal coming from twice as many deuterons in $\mathrm{CD}_{2}$ groups as $\mathrm{CD}$ groups in the 1,4 poly-butadiene arms as displayed in Fig. 6.17 where a monomer is pictured. The two spectra are overlaid in Fig. 6.18 and show no difference in linewidth or any splitting within the noise of the experiments $(<1 \%)$, which is an indication of a lack of physical alignment in the arms of the stars in the structure responsible for the banded state. The evidence presented here also suggests isotropic rotation on length scales of the order of the size of the central core of the stars. This raises an interesting question of how to resolve the dichotomy between the macroscopically jammed state and the freedom of movement that individual stars possess. It is likely that the answer lies in the structure of the deuterated stars. The per-deuterated arms are not deuterated all the way to the central junction. The process of making deuterated stars involves chemically fixing deuterated polymer arms to short, flexible, protonated arms which are themselves attached to the core. This structure means that the results presented in Fig. 6.18 are to be expected as the deuterium signal comes from arms that have no fixed 


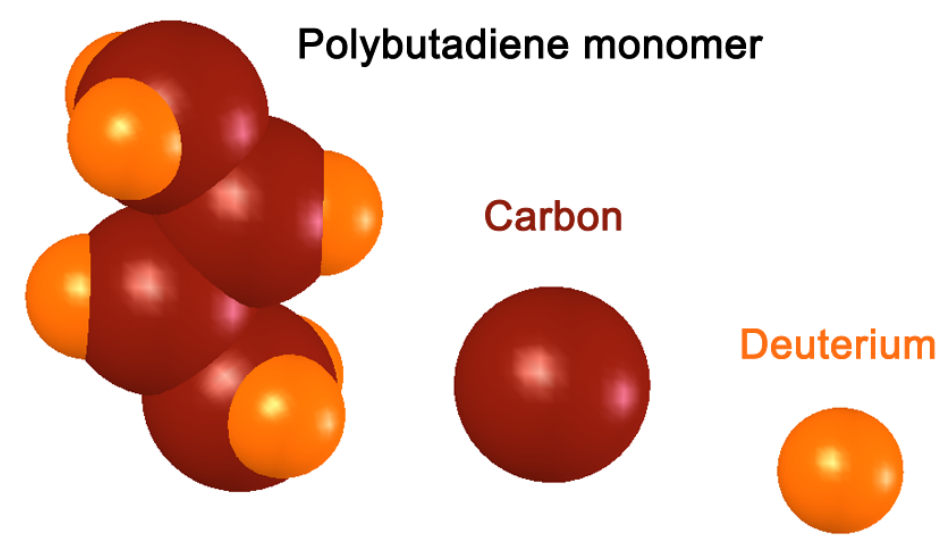

Figure 6.17: A graphical representation of a per-deuterated poly-butadiene monomer showing twice as many deuterons in $\mathrm{CD}_{2}$ environments as in $\mathrm{CD}$ environments.

point and are thus freely moving.

\subsection{Conclusions}

The results presented in this chapter support the idea of cluster formation in a suspension of stars in squalene. Velocimetry experiments have conclusively shown the evolution to a shear-banded state following fluidisation with the local shear rate of the low shear band being within the noise of the experiments. The growth of the 'zeroshear band' under shear can be described by the Gompertz equation, an equation that describes growth that is initially proportional to the size of the subject then slows and asymptotically approaches an upper limit. The effects of shear rate, waiting time and temperature on the Gompertz parameters allow some general conclusions to be drawn:

- There is a negative linear correlation between applied gap-average shear rate and long-time asymptotic extent of the zero-shear band. Changing the applied shear rate at any stage changes the long-time asymptote to that which corresponds to the new shear rate.

- The growth rate of the zero-shear band is proportional to the inverse of the applied shear rate, the time taken to reach unit strain. 


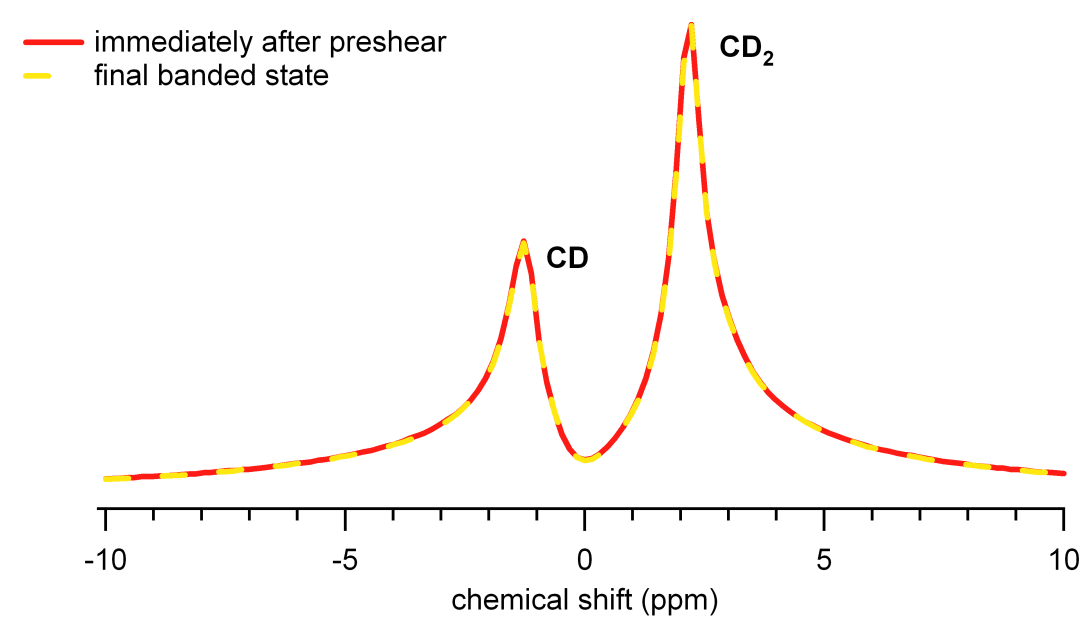

Figure 6.18: Comparison of the ${ }^{2} \mathrm{H}$ spectra immediately following preshear, that is, when the sample is completely fluidised, and after shearing at an imposed gap-average shear rate of $0.0314 \mathrm{~s}^{-1}$ for $36000 \mathrm{~s}$ so that the sample has reached the steady limit of the banded state. The spectra are identical to within the noise $(<1 \%)$ indicating a lack of alignment in the arms of the stars.

- There exists an exponential relation between the time of maximum growth and applied shear rate.

- Waiting time has no effect on the long-time asymptote of the extent of the zeroshear band.

- For waiting times $t_{w} \leq 100 \mathrm{~s}$ the growth rate is weakly negatively correlated to waiting time.

- For waiting times $t_{w}>100 \mathrm{~s}$ there is a power-law relation between waiting time and growth rate with an exponent of $0.69 \pm 0.04$.

- Increasing the waiting time has the effect of bringing forward the time of maximum growth up to a limit. Once that limit is exceeded, the size of the zero-shear band at $t^{\prime}=0$ will be greater than the noise. If sufficient time is allowed to elapse between preshear and the start of the experiment, the size of the zero-shear band will be greater than the long-time limit associated with the applied shear rate.

- Over a range of temperatures $290-333 K$ there is $1-\exp (-T)$ relation between the long-time limit of the extent of the zero-shear band and temperature with a 
temperature of $278 \pm 2.5 \mathrm{~K}$ corresponding to the point at which the zero-shear band does not exist.

- Over the same temperature range, there is an Arrhenius relation between growth rate and temperature with a prefactor $A=1426 \pm 180 \mathrm{~s}^{-1}$ and an activation energy $E_{a} / k_{B}=4530 \pm 280 \mathrm{~K}$.

- The time of maximum growth is proportional to $\left(T-T^{*}\right)^{-1}$ where $T^{*}=280 \pm$ $1 K$ is a shifted temperature origin that is equal, within the uncertainties, to that found from the long-time limit as a function of temperature.

- Individual stars are not deformed enough for ${ }^{2} \mathrm{H}-\mathrm{NMR}$ to show any change in the NMR spectrum from the fluidised state to the final state dominated by the zero-shear band.

Based on the data presented in this chapter, it is possible to examine the interplay between thermodynamic and kinetic effects on the evolution of a structure that manifests itself as the zero-shear band when sheared below a critical stress. In general, more thermal energy causes more of the sample to be included in the zero-shear band and also makes the zero-shear band grow faster and sooner. The data of Figs. 6.14 and 6.16 suggest that below a critical temperature of $T^{*} \sim 280 \pm 1 \mathrm{~K}$ there is not enough thermal energy for kinetic arrest to occur. Higher stresses caused by higher applied shear rates act to slow the growth and limit the extent of the zero-shear band. This interplay is akin to following the evolution of a chemical reaction where reactants and products form an equilibrium subject to two different rate constants: thermodynamics, which dictates the arm-disengagement rate, tends to push the system towards the zero-shear band (the 'product' end) and modified kinetics ('modified' in that only kinetics that involve stresses above the critical stress count), which is responsible for the breaking of cages, tends to restore fluidity (the 'reactants' end). This is a mesoscopic physical example of a molecular-level chemical phenomenon.

Ageing, and specifically ageing under shear, consists of a careful balance between thermodynamically-driven arm disengagement and kinetically-driven breaking of nearestneighbour cages. In the absence of shear, there is a natural hierarchy in timescales between the arm-disengagement rate and cage-breaking rate that is artificially altered by shearing. Ageing under shear will only proceed when the kinetics are sufficiently weak such that their magnitude is less than the magnitude of the thermodynamic effects. The 
rejuvenation process consists of driven kinetics with little or no thermodynamic component. Mechanical rejuvenation is not, therefore, the temporal reverse of ageing but rather a separate path in free-energy space. This idea is supported by the simulation work of Isner and Lacks [74] and the review by McKenna [75] which suggest that large mechanical stimuli do not entirely rejuvenate or erase all effects of ageing. The idea of different paths in free-energy space has previously been encountered in the work of Jabbari-Farouji et al. [103] and also in the previous chapter, 8, where it was suggested that there is a difference between the final states reached under oscillatory and continuous shear. The ideas presented in this chapter take the traversal of free-energy space thesis one step further and suggest that the preshear and ageing processes are themselves different paths that lead to reproducible initial and final states. 



\section{Chapter 7}

\section{A theoretical approach}

\subsection{Introduction}

The previous chapters have introduced the remarkable experimental behaviour exhibited by a solution of (nominally) 128-arm star polymers suspended in squalene. Using a well-defined preshearing protocol that places the system in a reproducible liquid state ("rejuvenation" of the system) it has been shown with conventional rheological techniques that the material evolves in a "two-step" manner [29]. The spatial resolution of rheo-NMR has shown that this two-step evolution manifests itself as a separation into bands of two widely different shear rates in a cylindrical Couette geometry.

It is apparent from the evolution of the flow curve from being monotonically increasing to one dominated by a stress plateau, see Fig. 5.8, and the response to creep experiments shown in Figs. 5.10 and 5.11 that the experimental parameter that affects the evolution of the mechanical response of the material is the applied stress. If the applied stress, $\sigma$, is below the critical stress, $\sigma_{c}$, then the material will solidify, while if $\sigma>\sigma_{c}$, the material will remain in the liquid state for all time. In this chapter, some theoretical approaches to modelling the data will be discussed that lead to a richer understanding of the physics present in this system.

The continuous evolution of the mechanical and dynamical properties of glassy fluids towards steady state has been the focus of much research. In particular, advancements have been made in the understanding of ageing effects with the trap model introduced by Bouchaud $[85,84]$ and later developed by Sollich et al. into the Soft Glassy Rheology (SGR) theory $[86,87,64]$. This chapter will begin with a brief review of their 
features and predictions.

\subsection{The trap model of Bouchaud}

Bouchaud $[85,84]$ suggested a simple picture that had been advocated by many authors $[104,105,106,107,108,109,110]$ prior, based on the idea that individual constituent particles of glass formers were 'caged' by their neighbours in a potential well. Bouchaud made the asumption that the 'cages' could be broken, i.e. the potential wells escaped from, by thermal activation alone. Bouchaud's model considers the thermal dynamics of independent particles in a space of traps characterised by a given probability distribution $\rho \varepsilon$ for the depth $\varepsilon$ of traps [85]. Activation in this model is purely thermally driven so that each particle may escape from its current trap of depth $\varepsilon$ with a rate of $\Gamma_{0} e^{-\varepsilon / k_{B} T}$, where $\Gamma_{0}$ is a constant 'attempt rate'. The depth of the new trap hopped into by a particle, $\varepsilon^{\prime}$, is arbitrarily chosen with probability $\rho\left(\varepsilon^{\prime}\right)$. The probability density, $P(\varepsilon, t)$, that a particle will be in a trap of depth $\varepsilon$ at a time $t$, evolves according to

$$
\frac{\partial P(\varepsilon, t)}{\partial t}=-\Gamma_{0} \exp \left(-\varepsilon / k_{B} T\right) P(\varepsilon, t)+\Gamma_{0} \omega(t) \rho(\varepsilon)
$$

The term $\omega(t)=\int \mathrm{d} \varepsilon P(\varepsilon, t) \exp \left(-\varepsilon / k_{B} T\right)$ is the average hopping rate at time $t$ and an initial condition of $P(\varepsilon, t=0)=P_{0}(\varepsilon)$ is imposed.

The steady-state solution to equation 7.1, if one exists, is given by $P_{e q}(\varepsilon) \propto \exp \left(\varepsilon / k_{B} T\right)$. The Boltzmann factor $\exp \left(\varepsilon / k_{B} T\right)$ is proportional to the average residence time of a particle in a trap of depth $\varepsilon$. If the density of traps has an exponential tail $\rho(\varepsilon) \approx$ $\exp \left(-\varepsilon / k_{B} T_{g}\right)$, then at a temperature $T=T_{g}$, the Boltzmann factor is canceled by the density of traps and the equilibrium probability distribution $P_{e q}(\varepsilon)$ tends to a constant for large $\varepsilon$, making it non-normalisable. The model thus predicts a glass transition at $T_{g}$. For temperatures $T \leq T_{g}$, the model has no steady state and evolves by 'ageing' into deeper and deeper traps; it is 'weakly' non-ergodic.

While the model of Bouchaud is able to propose a rather simple explanation of the glass transition, it does so with some major limitations for the study of soft glasses. Fundamental to the theory is the assumption that cages are broken/potential wells are escaped from by purely thermal processes. The activation barrier for the simplest local rearrangement in a foam, for example, is of the order of the surface energy of a single droplet. This sets a basic scale for the yield energies $\varepsilon$. Typical values for the surface 
tension of a droplet radius of the order of $1 \mu \mathrm{m}$ or greater gives potentials $\varepsilon \geq 10^{4} k_{B} T$. Related to this problem is that Bouchaud's model takes no account for strains, either micro- or macroscopic and therefore lacks the ability to effectively describe the kinetics of the system.

A theory based on Bouchaud's trap model that does take into account strains was proposed by Sollich et al. [86, 87, 64] and is known as the theory of 'Soft Glassy Rheology', (SGR).

\subsection{The SGR theory}

The SGR theory was proposed to account for similar rheological behaviours in a variety of soft glassy materials (SGMs): Herschel-Bulkeley or power-law equations are often used to describe the nonlinear flow behaviour $[2,111,112]$ and their viscoelastic storage and loss moduli, $G^{\prime}(\omega)$ and $G^{\prime \prime}(\omega)$, are often weak power laws of shear frequency $[113,114,115,78,116,117,118]$. The presence of similar behaviours in a wide range of SGMs is seen as being suggestive of a common cause. It has been argued [86,87] that such shared behaviours are symptomatic of common slow, glassy dynamics in these materials. The generic traits of such materials are noted as being structural disorder and metastability. To overcome the large energy barriers (compared to typical thermal energies) associated with structural rearrangements, it was proposed that energy came from a combination of thermal, strain and rearrangement processes. The denominator of the Boltzmann factor in Bouchaud's trap model was changed to an 'effective noise temperature', $x$, to account for these additional kinetic and energetic processes.

Sollich et al. noted that rather than working with individual particles, as Bouchaud had, their theory necessarily needed to involve mesoscopic 'elements' consisting of many constituents to ensure two conditions were satisfied: (i) the 'elements' must be small enough so that a macroscopic piece of the material contains so many of them that an ensemble average over elements may be used to describe the macrosopic properties; and (ii) the elements had to be large enough so that deformations of an element can be described by an elastic strain variable. An example is given in [87] of a single droplet in foam having a non-affine deformation and not being usable as an 'element'. In such a case, the size of an element would need to be several droplet diameters.

In addition to the 'noise temperature' that arises from a combination of thermal, strain and rearrangement processes, it was noted that straining an element increased 


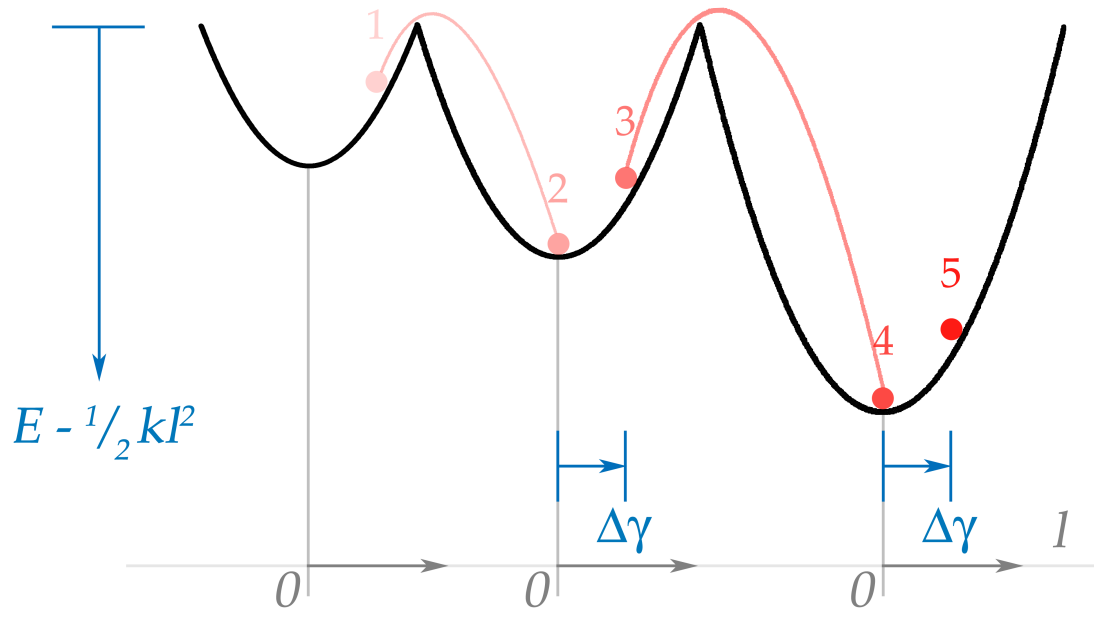

Figure 7.1: Illustration of the dynamics of the SGR theory. A representative element, 1, may hop out of its current trap by activated hopping. Macroscopic straining by an amount $\Delta \gamma$, lifts the energy $(2 \rightarrow 3)$ of the element, making a hopping event $(3 \rightarrow 4)$ more likely. The spatial positioning of the quadratic traps is arbitrary; each trap has a local strain origin at its centre. Successive traps are randomly selected from a prior distribution $\rho(\varepsilon)$.

its energetic position in a trap, taking a representative element closer to the edge of the trap and making a hopping even more likely. The second condition listed above allowed Sollich et al. to treat SGMs like elastic solids so that the energy is altered according to the square of the local strain, $l^{2}$, times the elastic constant, $k$ (it is further assumed that the local strain rate $i$ is equal to the macroscopic strain rate $\dot{\gamma}$ ). This further alters the Boltzmann factor so that the numerator is no longer just the depth of the trap an element is currently in, $\varepsilon$, but rather the difference between the depth of the trap and the energy given by straining, $\varepsilon-\frac{1}{2} k l^{2}$. The SGR theory thus treats SGMs as elastic solids with yielding events able to dissipate stress, giving a mechanism for flow to occur.

In a similar way to Bouchaud, Sollich et al. assumed that after a yield event, an element randomly finds a new trap from the density of traps $\rho(\varepsilon)$ and enters that trap in an unstrained position. This idea is illustrated in Fig. 7.1.

The probability of finding an element in a trap of depth $\varepsilon$ with local strain $l$ at a time $t$, according to SGR theory, evolves according to

$$
\frac{\partial P(\varepsilon, l, t)}{\partial t}=-\dot{\gamma} \frac{\partial P}{\partial l}-\Gamma_{0} \exp \left(-\left(\varepsilon-\frac{1}{2} k l^{2}\right) / x\right) P+\Gamma(t) \rho(\varepsilon) \delta(l)
$$


where $\Gamma(t)=\Gamma_{0} \int \mathrm{d} \varepsilon \mathrm{d} l P(\varepsilon, l, t) \exp \left(-\left(\varepsilon-\frac{1}{2} k l^{2}\right) / x\right)$ is the total yielding rate. The macroscopic stress is calculated by multiplying the (constant) elastic modulus by the average of the local strains:

$$
\sigma(t)=k\langle l\rangle_{P} \equiv k \int \mathrm{d} \varepsilon \mathrm{d} l P(\varepsilon, l, t) l .
$$

The activation processes that cause hopping between traps can be thought of as arising from either purely thermal processes, or from kinetic rearrangements elsewhere in the sample propagating through the material, causing further yield events. For this reason, the effective noise temperature, $x$, at the very heart of the SGR theory, is very difficult to write in a functional form [87]. It ought to be related to the shear rate and time: the majority of the energy $x$ represents comes from kinetic yield events propagating through the material and a higher shear rate necessarily causes more yield events per unit time. Under no strain, $x$ is predominantly thermal in composition. When strained, some elements will be in deeper traps simply because of the arbitrary choice of $\rho(\varepsilon)$ and thus take more straining before a yield event becomes likely. The effective noise temperature $x$ thus requires some sort of start-up of its own under steady shear conditions. When the strain is applied in a step-wise manner, $x$ ought to have some initially high value (or low if the system has aged significantly without shear), but decay off as a function of time as rearrangements become less likely due to the one-time application of strain.

In the units used by Sollich et al. a value of $x=1$ corresponds to the glass transition temperature. The model has different behaviours based on whether or not the effective noise temperature is greater than, or less than, the glass transition temperature. Without going into further details, it can be noted that early-time stresses in shear startup experiments (see Figs. 5.2, 5.4 and 5.5) are phenomenologically matched by theoretical simulations at an effective noise temperature $x=1.5$ (see Fig. 12 of [87]). Several features of the nonlinear oscillatory rheology presented in chapter 8 are also captured by SGR theory with an effective noise temperature of $x=1.1$, in particular the form of the dynamic moduli in a strain sweep (see Figs. 15 and 16 of [87] for example). SGR theory, with an effective noise temperature greater than the glass transition temperature, also predicts an increasing yield strain with frequency, an experimental effect noted in chapter 5 and in [29].

For all of the features of the experimental data presented in previous chapters on (nominally) 128-arm star polymers in squalene SGR theory offers explanations for, there is one glaring omission; the failure to offer an explanation of the two-stage evo- 
lution witnessed in rheological experiments.

In the fourth section of this chapter, a model that offers some explanation for the extraordinary experimental phenomena that has been the subject of the experimental chapters of this thesis is presented.

\subsection{A two-step model}

One potential problem with SGR theory is its requirement for an a priori definition of the density of traps. This requirement is identical to fixing the free-energy landscape regardless of the experimental conditions imposed. Assuming a fixed free-energy landscape may not physically make sense: deep traps correspond to tightly bound elements. If there is some yield stress inherent in the physical system, then an application of stress that exceeds the yield stress would eliminate the deepest traps, changing the free-energy landscape.

Motivated by the two-state evolution apparent in previous chapters, we suggest the following physical picture. The large stresses that arise during the oscillatory preshear ensure a fluidised state at the start of each experiment. In this state, the system flows homogeneously with a flow curve shown by 5.8. At stresses below the critical yield value $\sigma_{\mathrm{c}}$, however, this fluidised state is metastable. Under conditions of imposed shear rate $\dot{\gamma}<\dot{\gamma}_{c}$, this results in the formation of a solidified band that coexists with a fluid band at the critical stress $\sigma_{\mathrm{c}}$. At stresses above $\sigma_{\mathrm{c}}$, in contrast, the system remains fluidised. In summary: the system undergoes stress dependent switching between solidified and fluidised states, which can coexist under conditions of imposed shear rate.

A simple though useful guide to understand the interplay between nonlinear rheology and ageing properties for soft glassy materials was initially proposed by Derec et al. [63] and later developed by Picard et al. [119]. The model describes the local state of the system by a single scalar variable. For convenience, the scalar variable is taken to be the local relaxation frequency of the stress as expressed by a Maxwell equation

$$
\partial_{t} \sigma=G \dot{\gamma}-a^{2} \sigma .
$$

The first term on the right hand side of equation 7.4 corresponds to the elastic development of stress under an applied shear. The second term confers stress relaxation 
on a timescale $1 / a^{2}$. $a^{2}$ is referred to as the "fluidity" (it is squared to ensure it remains positive and hence physically meaningful) and it is the way it evolves that defines the dynamics of the model. To allow for shear banded states, the shear rate $\dot{\gamma}=\dot{\gamma}(y, t)$ is allowed to depend on the distance $y$ across the rheometer gap. The fluidity evolves as a competition between ageing processes and flow-induced rejuvenation so that $\partial_{t} a=-f(a)+g(a, \sigma, \dot{\gamma})$ where $f$ and $g$ are positive functions. The specific dynamics of the corresponding fluidity $a^{2}$ obey

$$
\partial_{t} a(y, t)=-M \frac{\delta V(\sigma, a)}{\delta a}+l^{2} \partial_{y}^{2} a+N(y, t) .
$$

Suppressing for the moment any spatial dependence upon $y$ such that $a=a(t), N=$ $N(t)$ only, at a fixed stress $\sigma$ the first term on the right hand side describes descent in an effective potential $V$ at a rate set by the mobility $M$. Alone, this term would ensure that the system descends until its first encounter with a local minimum of $V$, in which it would remain thereafter. The delta correlated noise $N$ models thermal and mechanical agitation, and confers a stochastic way of exploring the potential landscape such that the system can eventually find the global minimum. This is an important part of the way the theory works and warrants further discussion. It is the presence of the noise that allows the system to explore the potential landscape and hence to display the dynamics presented below. Without any noise present, or with not enough noise, the system simply remains in the first potential minimum it encounters. With too much noise, the system explores the potential landscape too rapidly and the dynamics of the model occur much quicker than the experiments suggest. The noise term in equation 7.5 needs to combine the Brownian movements and rearrangements caused by shearing in order to successfully model the system dynamics.

We show in Fig. 7.2 the form of $V$ chosen to match the two-state phenomenology seen in the experiments. To incorporate both fluidised and solidified states, we take

$$
V=\frac{a^{6}}{3}-\frac{[\alpha(\sigma)+\beta(\sigma)] a^{4}}{2}+\alpha(\sigma) \beta(\sigma) a^{2}
$$

with $0<\alpha<\beta$. By construction, this has a minimum representing a high fluidity state at $a^{2}=\beta$; and a minimum representing a solidified state at $a=0$. It can be shown that for $\beta$ less (resp. greater) than $3 \alpha$ the solidified (resp. fluidised) state is the global minimum. Stress dependent switching between these two states is captured by letting $\alpha=\alpha(\sigma)$ and $\beta=\beta(\sigma)$, with $\beta$ a more steeply increasing function of $\sigma$ such 
that switching occurs at the critical value of the stress $\sigma=\sigma_{c}$ for which $\beta=3 \alpha$. For convenience, we choose the following functional forms:

$$
\alpha=A+B \sigma^{n}, \text { and } \beta=B+B \sigma^{m},
$$

with $m>n$. From equations 7.6 and 7.7 it can be shown that $\sigma_{c}$ is the stress at which $\alpha=\beta / 3$ and in our parametrization takes the value $11.2 \mathrm{~Pa}$. The model thus eliminates one of the problems of the SGR theory by allowing the free-energy landscape to be stress dependent. Physically, what the model allows for, is the elimination of the solidified state when the stress in the system exceeds the yield stress.

While the basic phenomenology of the model is quite robust with respect to different values of the parameters $A, B, n, m$ and $l$, here specific choices are made: $A=$ $4.2 \times 10^{-3}, B=8.3 \times 10^{-3}, n=2.845, m=3.3, l=0.01$. These were obtained by first adjusting the parameters $A, B, n$ and $m$ to represent the two limiting flow curves displayed in Fig. 5.8, obtained respectively at the limits of short and long time, and $l$ along with the noise term $N$, to represent the time dependence of $\sigma(t)$ (Fig. 7.3). This noise is represented by a random value from a Gaussian distribution such that the standard deviation equals $\frac{1}{6}(\alpha-\beta)^{3}$, the height of the barrier between the two fluidity minima. By making such a choice of the functional form of the noise, the size of the barrier between fluidised and solidified states becomes the determining factor of the model's dynamics. The size of the barrier is always larger when attempting to leave the most energetically favourable state. This ensures that if a single element does leave the global minimum, it is more likely to make a hop over the (now smaller) energy barrier and re-enter the energetic minimum. This ensures the global minimum is the state most populated. By making the noise proportional to the stress experienced by the system, the model also accounts for the kinetics via rearrangement rates, a failure of the SGR theory of Sollich et al..

\subsubsection{Application of the model}

Having established the model, some features of the experimental data are now briefly reviewed along with the corresponding predictions of the model. In each experiment, a mechanical pre-shear protocol was followed comprising a large amplitude oscillatory shear $\left(\gamma_{0}=100 \%, \omega=1 \mathrm{rad} \mathrm{s}^{-1}\right)$ applied for a time $200 \mathrm{~s}$. This placed the system in a reproducible history-independent state. 


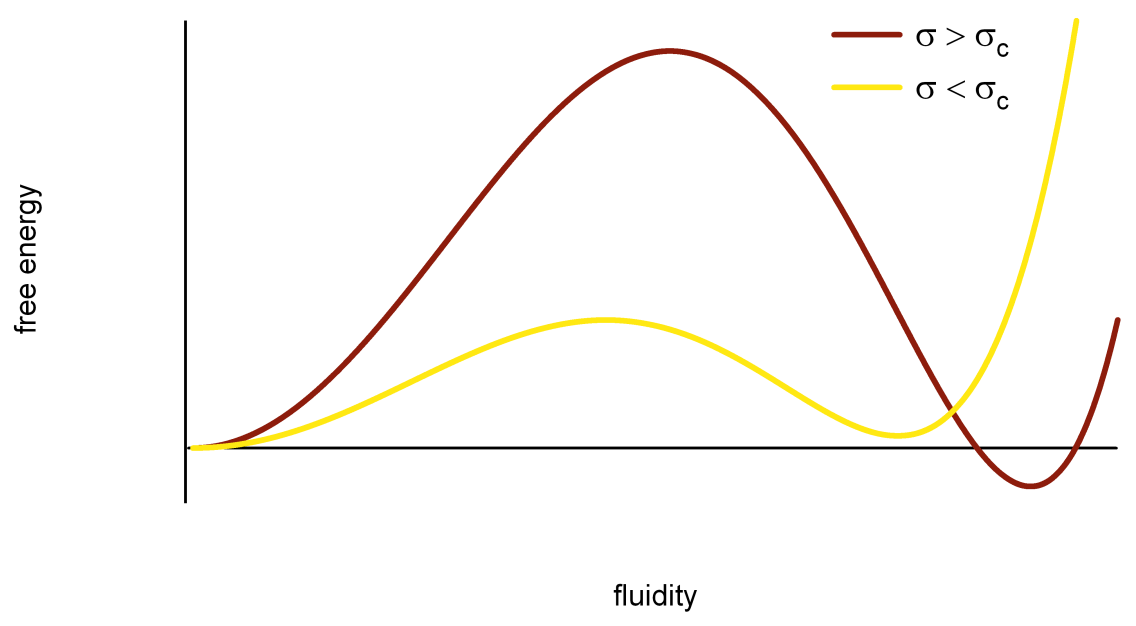

Figure 7.2: The shape of the free-energy-type function for stresses below and above the critical stress.

\section{Stress upon shear start-up}

The dotted lines in Fig. 7.3 are plots of the experimental stress response to a stepwise application of a shear rate for three different rates, $\dot{\gamma}=0.001 \mathrm{~s}^{-1}, 0.0025 \mathrm{~s}^{-1}$ and $0.05 \mathrm{~s}^{-1}$, performed on a strain-controlled ARES-HR rheometer equipped with a cone-and-plate geometry of diameter $25 \mathrm{~mm}$, angle $0.04 \mathrm{rad}$ and truncation $48 \mu \mathrm{m}$. For $\dot{\gamma}=0.001 \mathrm{~s}^{-1}$, the stress initially rises linearly with time/strain before reaching a metastable state where the stress, $\sigma(\dot{\gamma})$, is a function of the applied shear rate after $\sim 100 \mathrm{~s}$. For $\dot{\gamma}=0.0025 \mathrm{~s}^{-1}$, the stress overshoots to a plastic limit before relaxing back to the metastable state. The system remains in this metastable state for $\sim 2000 \mathrm{~s}$ before the stress begins to increase towards the equilibrium value of $\sim 11 \pm 0.25 \mathrm{~Pa}$. The duration of the metastable state increases with increasing shear rate up to a critical rate of $\dot{\gamma}_{c}=0.035 \mathrm{~s}^{-1}$. It is thought that the time spent in the metastable state, clearly a function of the shear rate, is dictated by caging and entanglement effects. Above $\dot{\gamma}_{c}$, the metastable state is indistinguishable from the stable equilibrium state. The dotted lines in Fig. 7.3 represent the experimental data and the solid lines correspond to the theoretical predictions. It is easy to see that while the stress overshoot at short times is not captured by the model the two-step stress evolution is well described. 


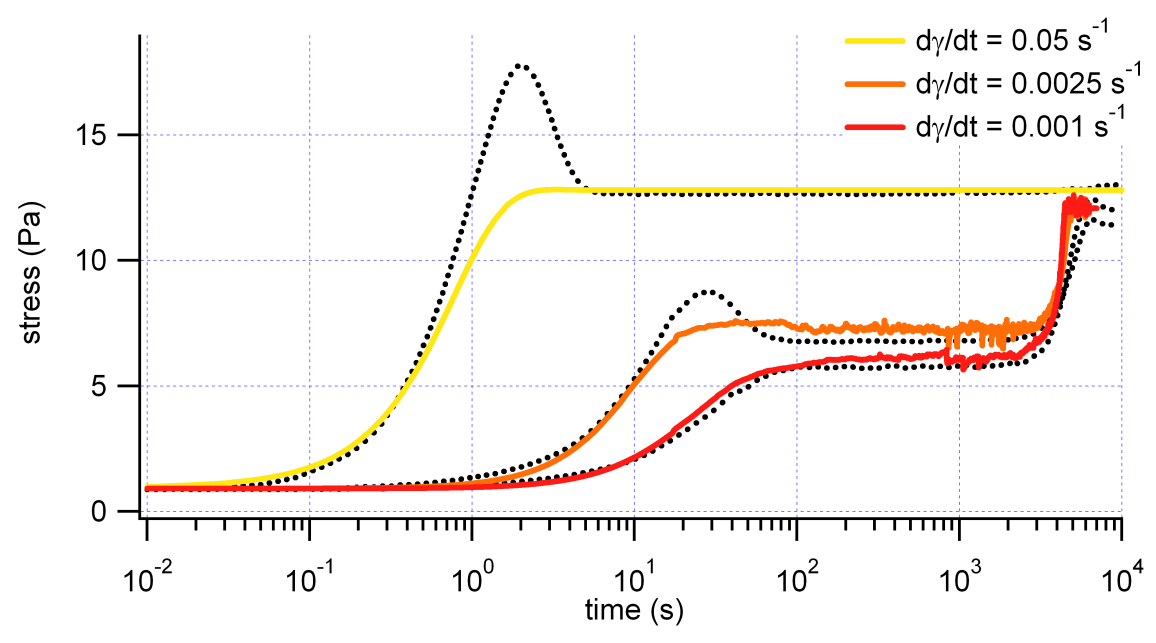

Figure 7.3: Dotted black lines: Experimental stress evolution upon shear startup immediately following preshear, measured in a cone-and-plate geometry for the star polymer system and for shear rates $\dot{\gamma}=0.001 \mathrm{~s}^{-1}$ (lower line) and $\dot{\gamma}=0.0025 \mathrm{~s}^{-1}$ (middle line) and $\dot{\gamma}=0.05 \mathrm{~s}^{-1}$ (upper line). Coloured lines: Behaviour of the phenomenological model under identical shear conditions.

\section{Evolution of the flow curve}

Figure 7.4 shows a closeup of the flow curve (full curve displayed in Fig. 5.8), measured in a downward rate sweep started at a time $t_{w}=0^{*}$ after the preshear $\left(0^{*}\right.$ denotes a small delay, set by the control software, being between the end of the preshear and the beginning of the rate sweep). At a shear rate sweep residence time of $10 \mathrm{~s}$ per point, (yellow crosses) the flow curve indicates the material behaves like a shearthinning fluid. Figure 7.3 indicates that a residence time of $10 \mathrm{~s}$ samples the stress before reaching the metastable state at low shear rates. Having also seen from Fig. 7.3 that the stress takes on the order of $10000 s$ to reach the stable state we show a second rate sweep where the residence time is also of the order of $10000 s$ (dark red crosses). What is apparent is a stress plateau that develops after thousands of seconds, with the plateau taking longer to reach as $\dot{\gamma}$ approaches $\dot{\gamma}_{c}$ from below. At intermediate times when the plateau is not fully formed, the flow curve exhibits a local minimum that can appear as a viscosity bifurcation in stress-controlled tests. When sufficient time has elapsed that the plateau is fully formed, no such bifurcation exists. Rates lower than $10^{-4} s^{-1}$ are difficult to access with the controlled strain rheometer. This evolutionary behaviour is contrasted with the case of worm-like micelles where any stress plateaux form rapidly. The solid lines in Fig. 7.4 are the theoretical predictions for identical con- 


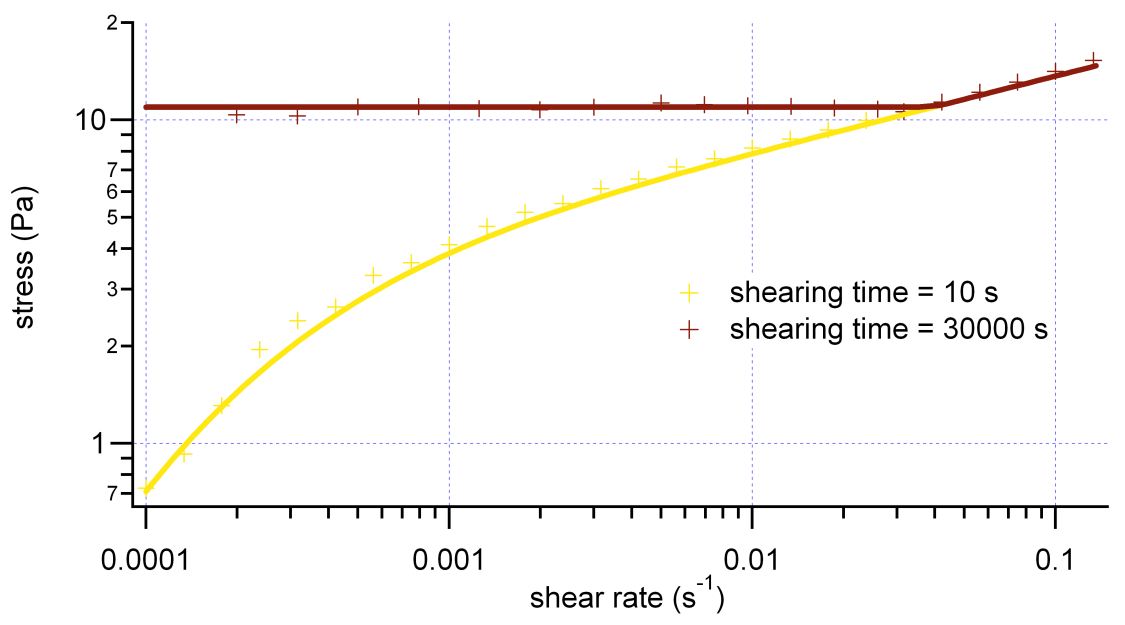

Figure 7.4: Yellow crosses: Flow curve measured in cone-and-plate geometry on the star polymer system with a residence time of $10 s$ per point. Dark red circles: Flow curve where the residence time is on the order of $30000 \mathrm{~s}$. Solid lines: Behaviour of the phenomenological model under identical conditions.

ditions to the experimental data, shown as crosses. The plateau and the evolution are well described by the model.

\section{A note on the possible lower branch of the flow curve}

Creep experiments (see Figs. 5.10 and 5.11) suggest a lower branch of the flow curve exists around $10^{-5} s^{-1}-10^{-6} s^{-1}$, visible by slow accumulation of strain at long times for stresses $\sigma<\sigma_{c}$. This is accounted for by the model via a small value of the fluidity. The small value is a result of each element finding its way into the global potential minimum located at a fluidity of $a=0$ and having small 'boosts' in energy from the effective noise inherent in the system (a combination of thermal and kinetic processes). In such a state, the noise would be predominantly thermal in nature due to a lack of rearrangements taking place. A series of creep experiments where the applied stress is below the critical yield stress would thus elucidate the lower branch, and hence, the amount of thermal noise in the system.

\section{Banded velocity profiles from rheo-NMR}

Shear banding is not peculiar to glassy materials, but has been observed in worm-like micelles [66, 67], emulsions near their yield stress [68], entangled polymer solutions 
[69], liquid crystalline polymers [70] and recently in a nearly random close packed, hard-sphere colloidal suspension [71]. Shear banding has also been reported in dense pastes of large $(0.29 \mathrm{~mm})$ polystyrene beads [72]. Shear banding, identified experimentally through the coexistence of two or more bands of different viscosity or microstructure, is typically accounted for theoretically by a constitutive flow curve with an unstable branch of negative slope. When shear-rate conditions that correspond to the region of unstable flow are imposed, these systems can separate into phases of different local shear rates that coexist at a common stress. A recent review by Moller et al. [73] suggests shear banding and thixotropy can be viewed as two effects of the same underlying cause. The fascinating question as to whether non-monotonic constitutive behaviour might be inherent to glassy yield-stress systems, remains open.

Stress plateaux in flow curves have been linked to the phenomenon of shear banding. Figure 7.5 shows two velocity profiles across the $1.5 \mathrm{~mm}$ gap of a cylindrical Couette rheo-NMR cell at an apparent shear rate of $0.0314 \mathrm{~s}^{-1}$, just below the critical strain rate, and obtained at different times following the preshear protocol. The cell has inner and outer radii of $7.5 \mathrm{~mm}$ and $9 \mathrm{~mm}$. The yellow crosses show the velocity profile across the cell at time $t^{\prime}=150 \mathrm{~s}$ which corresponds to the yellow crosses of Fig. 7.4. The dark red crosses show the velocity profile at $t^{\prime}=25000 \mathrm{~s}$ and reveal a banded structure where the lower shear rate band has a shear rate $\sim 0 s^{-1}$. The small value of the shear rate indicates an increase in the viscosity, similar to that observed by Coussot et al. [120]. However, the data of Fig. 7.3, in particular the lack of thixotropy, the stress overshoot and the long time before stiffening, suggests that different physics is responsible. The central cylinder is filled with a marker fluid so the inner wall velocity is known. No slip is seen during the evolution. The low shear rate band evolves across the gap from the outer wall to reach a final fraction dictated by the applied shear rate.

The predictive power of the model can be tested by using the previously listed parameter set to generate the velocity profiles of Fig. 7.5. The description of shear banding at an imposed shear rate $\overline{\dot{\gamma}}=\int \mathrm{d} y \dot{\gamma}(y, t)$ requires the term $l^{2} \partial_{y}^{2} a$ to correctly represent the structure of the interface between the bands, with a characteristic width $l$, expressed as a fraction of the gap traversed by $y$. As shown in Figs. 7.3 and 7.4 the model captures the basic phenomenology, and further, predicts good agreement with the velocimetry data shown in Fig. 7.5. In a shear startup experiment, and following the pre-shear protocol, (Figs. 7.3 and 7.5) the stress attains the fluidised branch of the flow curve, on which the system flows homogeneously. For values of the shear rate $\overline{\dot{\gamma}}<$ 


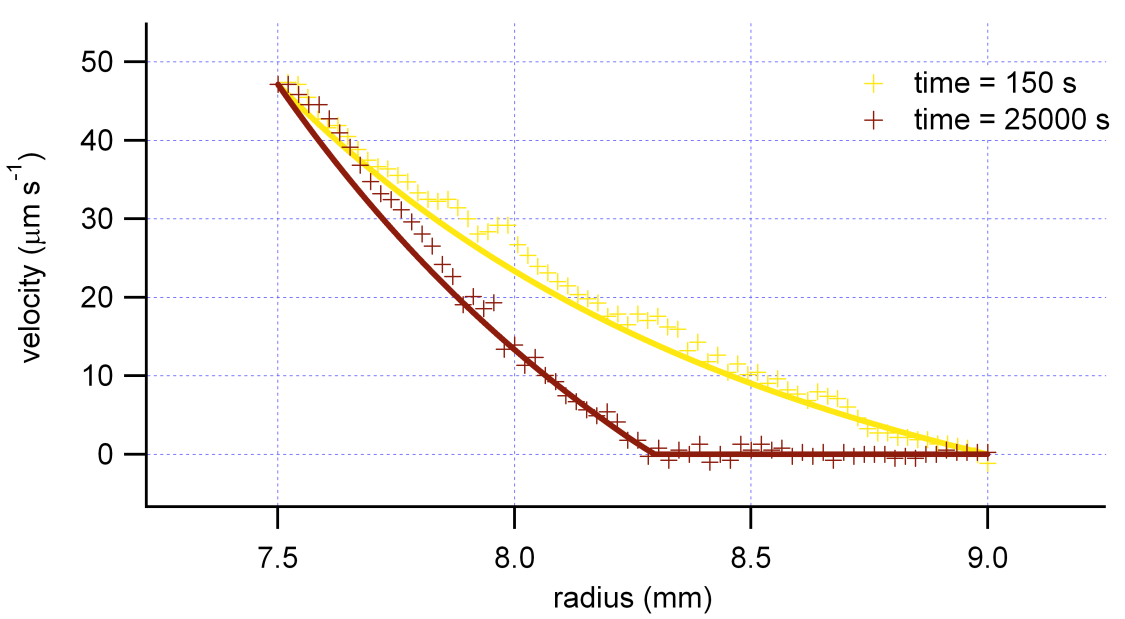

Figure 7.5: Yellow crosses: Velocity profile across the $1.5 \mathrm{~mm}$ gap of the cylindrical Couette at an apparent shear rate of $0.0314 \mathrm{~s}^{-1}$ at time $t^{\prime}=150 \mathrm{~s}$ following preshear. Dark red crosses: Velocity profile under the same conditions at a time $t^{\prime}=25000 \mathrm{~s}$. Solid lines: Behaviour of the phenomenological model under identical conditions.

$\dot{\gamma}_{\mathrm{c}}$, the stress subsequently evolves towards its final value $\sigma_{\mathrm{c}}$, at which a solidified band coexists with a fluid band. Likewise in a shear rate sweep (Fig. 7.4), the model predicts the fluidised homogeneous flow branch shown by the yellow line for fast sweep rates; and a yield stress at which fluidised and solidified bands coexist for slow sweep rates.

\subsection{Conclusions}

The model system examined here manifests a two-step ageing property that may underpin a wide class of yield-stress fluids. The dynamics of the model are dictated by a combination of stress-dependent parameters: the free-energy landscape alters as a function of stress, making the solidified state less likely at higher stresses, and an effective noise that depends on the stress, reflecting the physical kinetic nature of rearrangement rates. That the time-dependence of both the stress and shear rate profile on steady shear start-up, as well as the time-dependent flow curve is captured by using such a simple phenomenological model suggests the possibility of a universal description for ageing-rejuvenation and shear banding dynamics in soft glasses. 



\section{Chapter 8}

\section{Large-Amplitude Oscillatory Shear (LAOS) Response}

\subsection{Introduction}

A Lissajous curve is the graph of a system of parametric equations which describe complex harmonic motion:

$$
\begin{aligned}
& x(t)=A \sin (a t+\delta) \\
& y(t)=B \sin (b t) .
\end{aligned}
$$

These curves were initially studied by the American mathematician Nathaniel Bowditch in the early 19th century who created them by means of a pendulum suspended from two points. In the mid-19th century French mathematician Jules Antoine Lissajous studied the curves in more detail and they now bear his name. Lissajous was interested in waves and created an apparatus where a beam of light was reflected off a mirror attached to a vibrating tuning fork and then off another mirror attached to a perpendicularly vibrating second tuning fork and then onto a wall creating a 'Lissajous figure'. Lissajous curves can take the form of lines or closed loops. A Lissajous curve will be closed, if and only if, the ratio of frequencies, $a / b$ is rational.

In the rheological literature a Lissajous curve is typically a parametric plot of the oscillating stress response as a function of the sinusoidally oscillating strain input. In this case, a purely elastic response is a straight line of gradient $G$ and a purely viscous response is elliptical with minor/major axes along the stress/strain axes. The ratio 


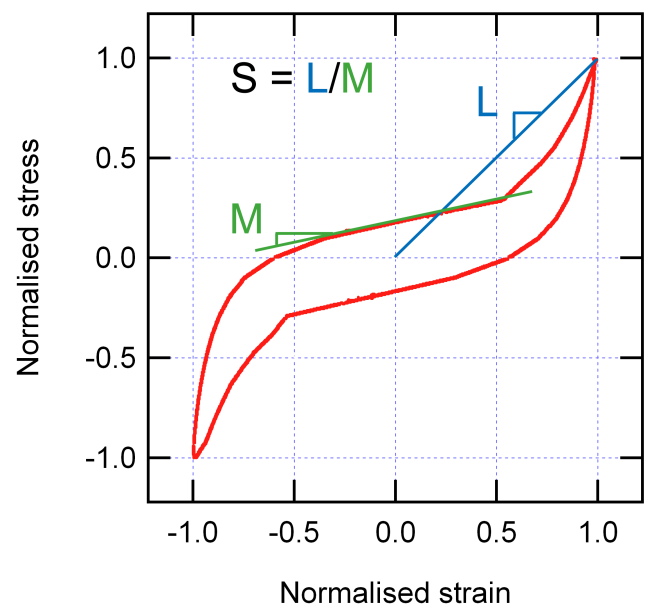

Figure 8.1: Typical Lissajous curve of the response of gastropod pedal mucus to oscillatory strains taken from the MS thesis of Randy Ewoldt [121] showing how the elastic stiffening ratio is calculated.

of major-to-minor axis is equal to the product of the angular frequency and viscosity, $\omega \eta$. Equally, a purely elastic response is in phase with the oscillating strain and a purely viscous response is in phase with the oscillating strain-rate (which oscillates at the same frequency as the strain and leads by $\pi / 2 \mathrm{rad}$ ). A viscoelastic response is a combination of these behaviours and forms an ellipse in a Lissajous plot. If the stress is plotted as a function not of strain but of strain-rate, then the Lissajous curve is an 'oscillatory flow curve' where the ratio of stress to strain-rate at any part of the curve is the instantaneous viscosity. Any nonlinearities distort the ellipse. The main goal of any quantitative analysis technique is to characterise the nonlinearities present in the response of the material being studied. Several methods of analysis are currently in the literature.

The method of Ewoldt et al. [121] consists of measuring the ratio of the largestrain elastic modulus, $L \equiv\left(\left.\tau\right|_{\gamma= \pm \gamma_{0}}\right) /\left( \pm \gamma_{0}\right)$, to the small-strain elastic modulus, $M \equiv$ $(d \tau) /\left(\left.d \gamma\right|_{\gamma=0}\right)$. This result, called the elastic-stiffening ratio $S\left(\omega, \gamma_{0}\right) \equiv L\left(\omega, \gamma_{0}\right) / M\left(\omega, \gamma_{0}\right)$ gives an indication of the amount of strain-stiffening that takes place. A value $S>1$ indicates strain-stiffening behaviour and a value $S<1$ indicates strain-softening behaviour. This particular analysis method works well for the hysteresis-type responses seen in the application of oscillatory strains to gastropod pedal mucus illustrated in Fig. 8.1.

The method of Klein et al. [122] assumes a more general approach. The approach 
is to characterize the stress response in terms of an addition of four basis states called characteristic responses: sine wave, rectangular wave, triangle wave and sawtooth wave. To each of these contributions a physical process is attached: a sine wave represents linear behaviour, a rectangular wave corresponds to strain softening, a triangular wave component reflects the strain hardening and a sawtooth wave is used to model shear bands. The analysis then follows as a direct fitting of these four characteristic responses varying the amplitude, frequency and phase of each response to fit the time domain data as well as the frequency domain data. This approach assumes ideal behaviours for all the specified physical processes.

Cho et al. [123] describe the stress response of a material subjected to LAOS via two derived quantities referred to as the elastic and viscous stress components and denoted $\sigma^{\prime}$ and $\sigma^{\prime \prime}$ respectively. Cho et al. use a formalism where $x=\gamma$ and $y=\dot{\gamma} / \omega$ and invoke an assumed symmetry of response, $\sigma(-x,-y)=-\sigma(x, y)$, to derive the quantities $\sigma^{\prime}=[\sigma(x, y)-\sigma(-x, y)] / 2$ and $\sigma^{\prime \prime}=[\sigma(x, y)-\sigma(x,-y)] / 2$ where $\sigma^{\prime}+\sigma^{\prime \prime}=\sigma$. These quantities are used to define generalised dynamic moduli, $\Gamma^{\prime}$ and $\Gamma^{\prime \prime}$, via the relations $\sigma^{\prime}=\Gamma^{\prime}\left(x, \gamma_{0}\right) x, \sigma \prime \prime=\Gamma^{\prime \prime}\left(y, \gamma_{0}\right) y$. In the linear viscoelastic limit, $\lim _{\gamma_{0} \rightarrow 0} \Gamma^{\prime}\left(x, \gamma_{0}\right)=$ $G^{\prime}(\omega), \lim _{\gamma_{0} \rightarrow 0} \Gamma^{\prime \prime}\left(y, \gamma_{0}\right)=G^{\prime \prime}(\omega)$. McKinley and Ewoldt [124] have recently suggested using Chebyshev polynomials of the first kind (an orthogonal set of polynomials) to model the elastic and viscous stresses. The benefit of this approach is that unambiguous coefficients of the orthogonal Chebyshev polynomial basis set are acquired, leading to a physical interpretation that a direct Fourier transform of the raw data cannot give.

The system of star polymers studied here has a very rich nonlinear rheology. A typical normalised stress response to an oscillatory strain $\left(\omega=1 \mathrm{rad} \mathrm{s}^{-1}, \gamma_{0}=20 \%\right)$ is shown in Fig. 8.2 as a function of time and as two Lissajous curves (normalised stress as a function of normalised strain and as a function of normalised strain rate).

The most striking feature of the data presented in Fig. 8.2 is the concavity of the response at $\max / \min$ stresses. At strain amplitudes $\gamma_{0} \geq 20 \%$ the small strain elastic modulus $M \equiv d \tau /\left.d \gamma\right|_{\gamma=0}$ is negative or zero, which, according to the method of Ewoldt et al. [121], would give the unphysical results of negative or infinite elastic stiffening ratios. The approach of Klein et al. has been shown in [122] to give a reasonable fit to the oscillatory-stress response of dispersions of monomer of size between 50 and $500 \mathrm{~nm}$ in a continuous water phase. The result of the application of this method to the experimental data of Fig. 8.2 is shown in Fig. 8.3. While the method of Klein et al. [122] 

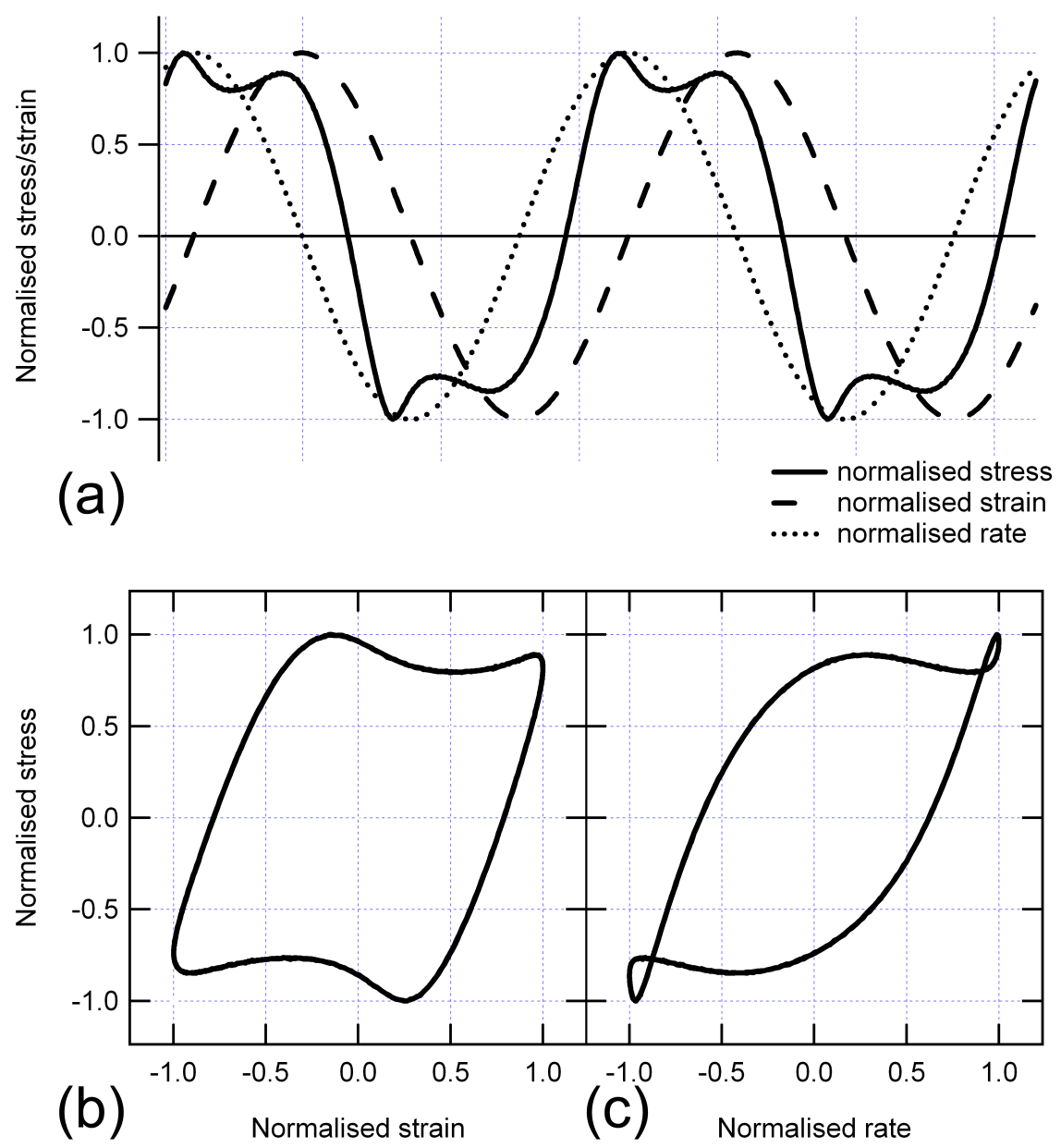

Figure 8.2: (a) The normalised stress response of star polymers (solid line) to an oscillatory strain of $20 \%$ as a function of time. Normalised oscillatory strain (dashed line) and strain rate (dotted line) are shown also. (b) Lissajous curve of the normalised stress as a function of normalised strain. Note the concavity at $\mathrm{max} / \mathrm{min}$ stresses. (c) 'Oscillatory flow curve': Lissajous curve of the normalised stress as a function of the normalised strain rate. Note that the two Lissajous curves are traced in alternate directions; the stress as a function of strain, shown in (b), progresses clockwise and when plotted against strain rate as shown in (c), the curve is traced out in an anticlockwise direction. 


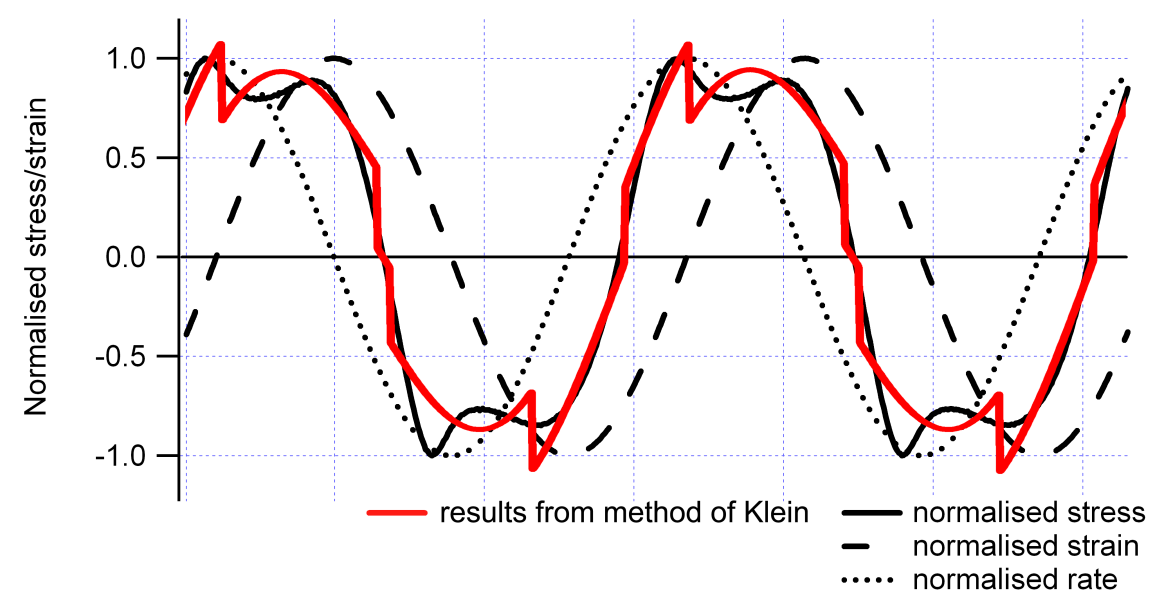

Figure 8.3: The method of Klein et al. applied to the data of Fig. 8.2. The solid black line is the experimental data of Fig. 8.2 and the coloured line is a combination of a sine wave representing linear behaviour, a rectangular wave representing strain softening, a triangular wave representing strain hardening and a sawtooth wave representing slip/shear bands according to the method of Klein et al. This method does not account for the shape of the response observed experimentally.

is able to produce a stress decrease, it does so in a discontinuous manner and fails to match the symmetry of the response. In general, this method falls significantly short of reproducing the smooth stress response.

It is universally assumed that the response of materials to small- and large-amplitude oscillatory shear display particular types of symmetry. The material responses are assumed to belong to a class of mathematical objects known as friezes. They are named after the friezes occurring in art where decorations are obtained by repeating a symmetric or antisymmetric motif. In an attempt to assist the reader in understanding some of the fundamental points of this chapter, appendix A introduces some of the mathematical properties of frieze groups and introduce Conway's [125] orbifold notation.

\subsection{Proposed method}

The methods of Ewoldt et al. and of Klein et al. characterise the oscillatory shape of the stress response in terms of non-linearities. In the method of Ewoldt et al. this happens via the determinism of a single parameter that relates the large- to small-strain elastic 
moduli. In the method of Klein et al. the nonlinear response comes from the non-zero amplitudes of the nonlinear basis states which gives information about the relative intensities of four idealised responses.

In an oscillatory experiment there is a continuous range of shear rates being traversed with an amplitude of $\gamma_{0} \omega$. The long-time, low-frequency, large amplitude limit of an oscillated strain rate experiment produces the flow curve of the material. A shorttime, high-frequency, small amplitude experiment can be thought of as producing an approximation to the steady-state flow curve, or better yet, as elucidating a version of the flow curve at an earlier time in its evolution. Thus, it is reasonable to use the considerable knowledge of constitutive curves (model flow curves) in the literature to model the stress response to oscillatory strains. Regardless of the approximation being valid, it is still a worthwhile exercise to determine the flow curves traversed in oscillatory experiments. This is essentially the current proposal; instead of starting the analysis by trying to quantify the amount of nonlinear behaviour, we first find the form of the underlying 'oscillatory flow curve' that is followed. Thus, a series of oscillatory experiments of various strain amplitudes and frequencies can map out the early-time evolution of what becomes the steady-state flow curve. This is effectively an extension of the Cox-Merz rule. The Cox-Merz rule is an empirical rule that states the flow curve obtained by oscillatory experiments is essentially the same as the flow curve obtained from steady-state shear experiments. Indeed, in the long-time, low frequency, large amplitude limit, this is exactly what happens. The usefulness of the Cox-Merz rule is in the time required to obtain each 'version' of the flow curve; a single, large amplitude oscillatory experiment can produce a flow curve that requires many steady-shear experiments. The current proposal extends the idea of the Cox-Merz rule to suggest that the entire evolution of the flow curve may be mapped by a series of oscillatory experiments rather than only elucidating the final steady-state flow curve with a single oscillatory experiment. With this idea in mind, the partly concave shape formed by the data of Fig. 8.2 suggests a stress response that is characteristic of a flow curve being traversed that contains a branch of negative slope. A simple flow equation that allows for a negative branch was proposed by Cross [6] and is based on the assumption that pseudoplastic flow is associated with the formation and rupture of structural linkages. The exact form of the Cross equation is

$$
\eta=\eta_{\infty}+\left(\frac{\eta_{0}-\eta_{\infty}}{1+\alpha \dot{\gamma}^{n}}\right)
$$



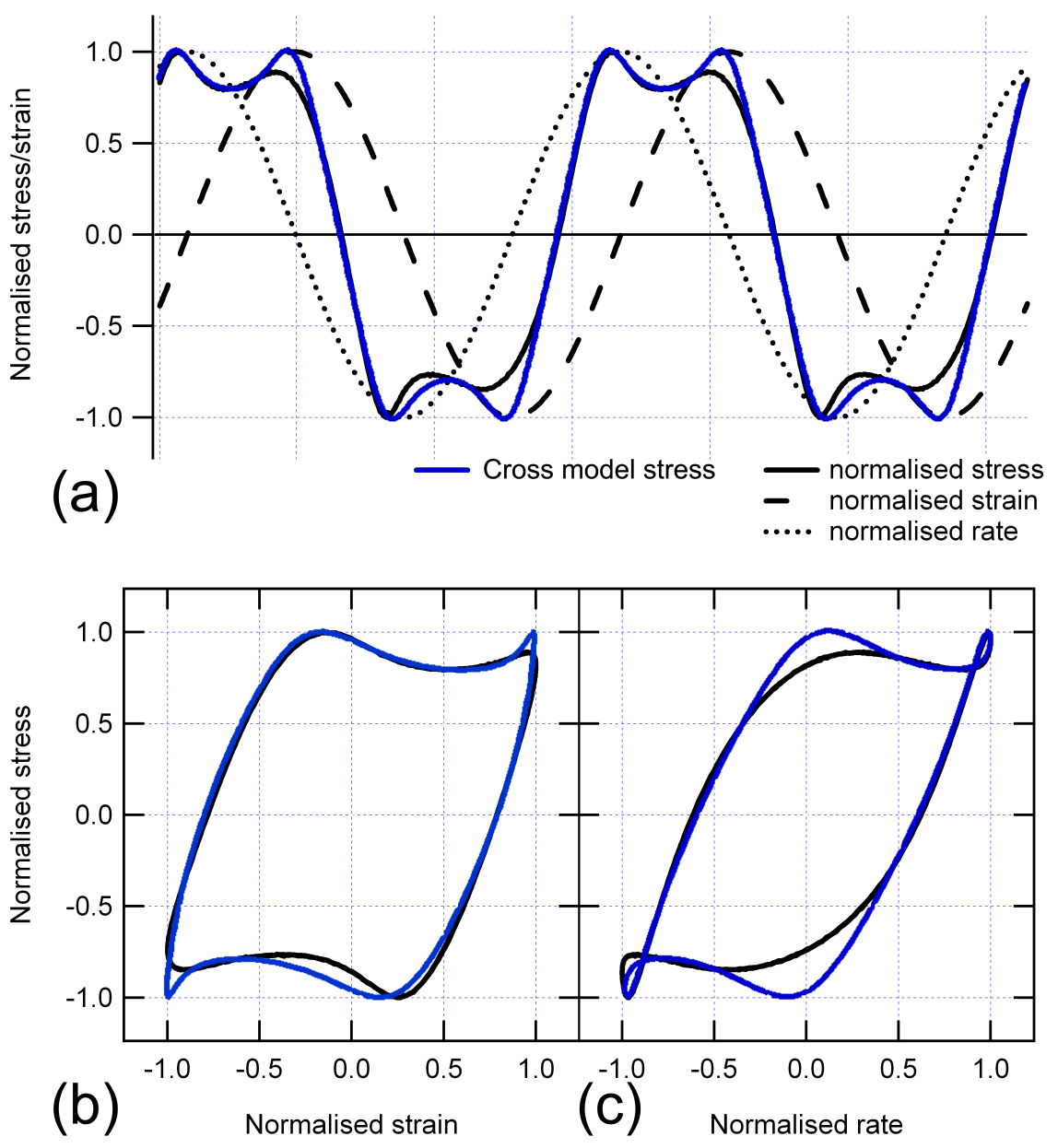

Figure 8.4: A fit to the data of Fig. 8.2 using the Cross model of equation 8.1. The phenomenology of the stress decrease is well modelled in this way.

where $\alpha \gg 1$ implies a relatively large shear dependent contribution to structural breakdown and the other symbols have their usual meanings. Figure 8.4 shows the result of using this equation with parameters $\eta_{\infty}=141 \mathrm{Pas}, \eta_{0}=37 \mathrm{Pas}, \alpha=6$ and $n=6$, with a phase shift incorporated into the strain-rate term to model the experimental data of Fig. 8.2. The phenomenology of the stress decrease is well modelled in this way, although the Cross model produces a $2 * \infty$ (spinning sidle) frieze group, where the data more closely matches an $\infty x$ (step) frieze group.

We note here that the equation presented by Cross, equation 8.1, was derived as the steady state representation of some presumed dynamics and was not originally intended to model any transients. That the Cross model describes the material response 
to LAOS presented here suggests that the same dynamics may be present in the physical processes that lead eventually to the steady flow state. Equation 8.1 was derived from the assumption that linkages between material constituents form via Brownian movements and that effective rate constant for the rupture of those same linkages by shearing is a power-law. Cross argues further

"that the rate of rupture of linkages must be an even function of the shear rate because it must be independent of the direction of applied shear"

. This idea is investigated in more depth in following sections.

It is apparent from the data displayed in Fig. 8.2 that there does not exist a vertical line of symmetry for each stress lobe, that is, the data do not constitute a $2 * \infty$ (spinning sidle) frieze group. This lack of symmetry suggests that the upward stress sweep is different to the downward stress sweep. That is, a different flow curve is traversed upwards to the one followed on the downwards sweep. The upward stress sweep causes structural rearrangements in reaching the maximum strain rate and so the downward sweep necessarily follows a different stress path. The fitting procedure may then be broken into two parts, modelling the upward rate sweep separately from the downward in order to produce an $\infty x$ (step) frieze group. This approach is demonstrated in Figs. 8.5 and 8.6 with the 'active' flow curve shown in dark blue and the 'inactive' curve in light blue. The parameters that give the lower curve are $\eta_{\infty}=113 \mathrm{Pas}, \eta_{0}=29 \mathrm{Pas}, \alpha=5.4$ and $n=6$. These are specifically chosen to reproduce the experimental LAOS data. Note the flow curve that is being traversed is only elucidated as far as the maximum shear rate achieved in the experiment and any part of the flow curve that is higher than the maximum shear rate, in the case of Figs. 8.5 and 8.6, of $\gamma_{0} \omega=0.20 \mathrm{~s}^{-1}$, must be viewed as conjecture only and not supported by experimental data.

The progression of the red line in successive rows of Fig. 8.5 indicates the passage of time. In the top two rows of Fig. 8.5, the first stress peak is successfully modelled by the higher flow curve shown in dark blue. The 'inactive' flow curve, the one followed on a downward sweep, is shown in light blue. When the strain reaches the maximum, the active flow curve switches and the lower stress sweep follows the lower flow curve. Both stress peaks are well described by this switching procedure.

Figure 8.6 illustrates the changing of the active flow curve followed when the strain reaches zero again. 

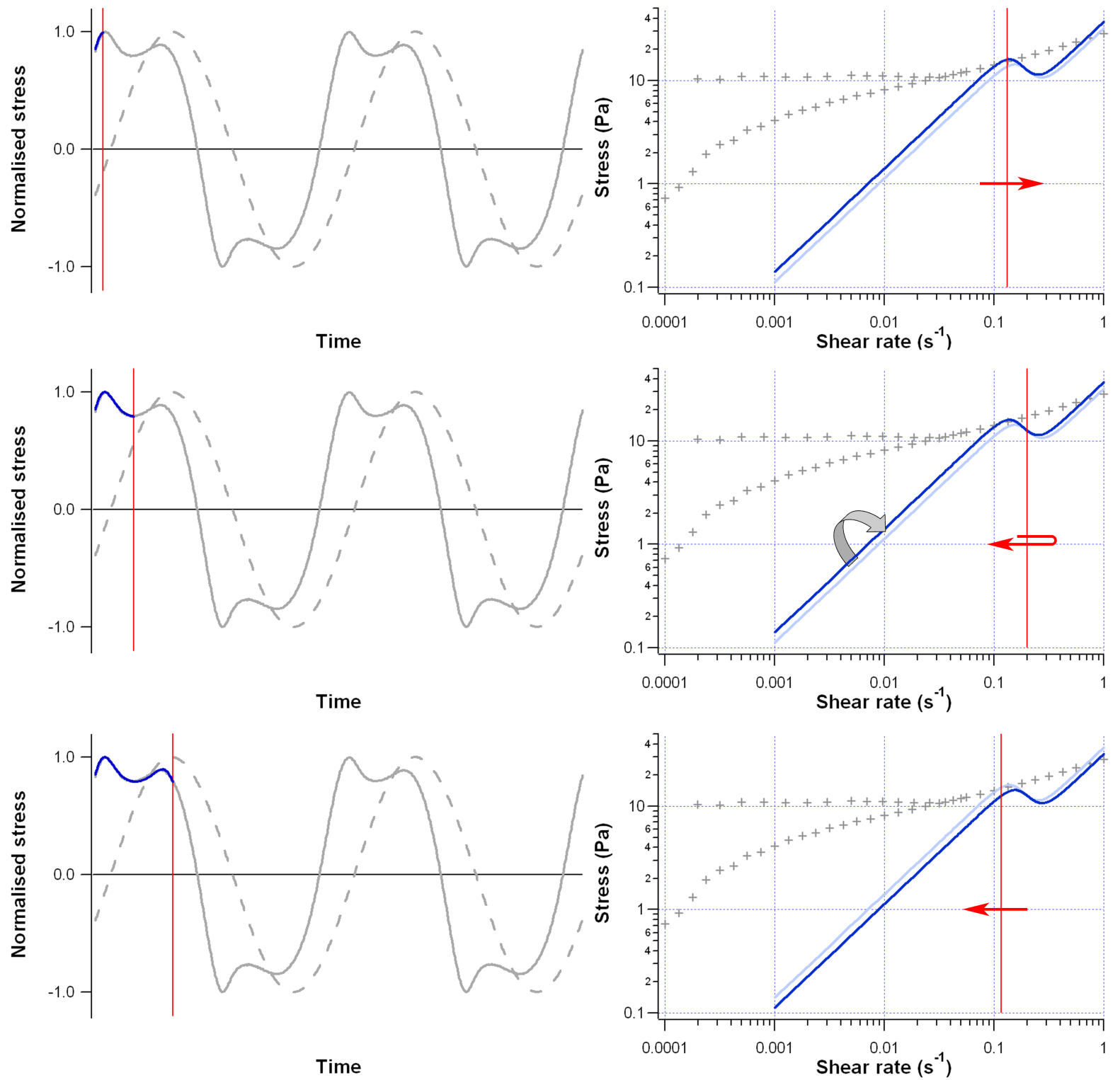

Figure 8.5: The experimental data of Fig. 8.2 in grey with the fit from the method outlined in the text in blue. The three rows correspond to different times in the experiment and the right column shows the stress decrease in the flow curve. A section of the non-monotonic branch of the flow curve is traversed in the course of an experiment which causes the characteristic concave shape of the stress response shown on the left. To account for the change in stress peaks seen, the downward rate sweep is assumed to follow a different flow curve as shown in the right column. The grey crosses shown in the flow curve are the measured flow curves of residence times $10 s$ and $\sim 10000 s$. 


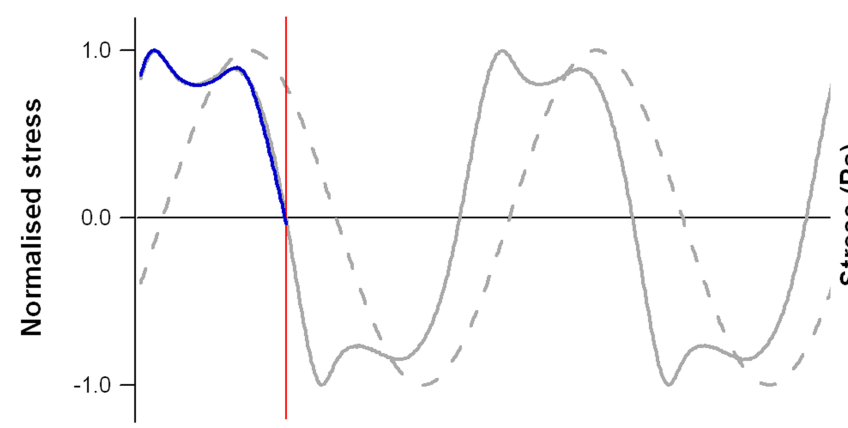

Time

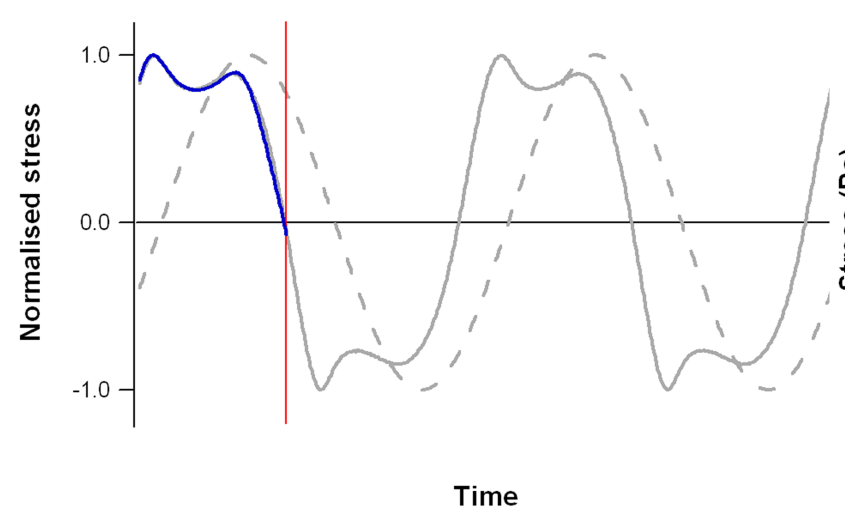

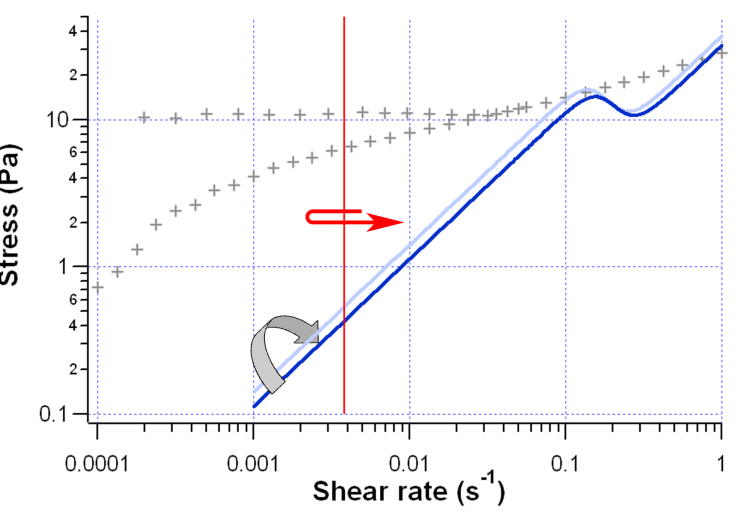

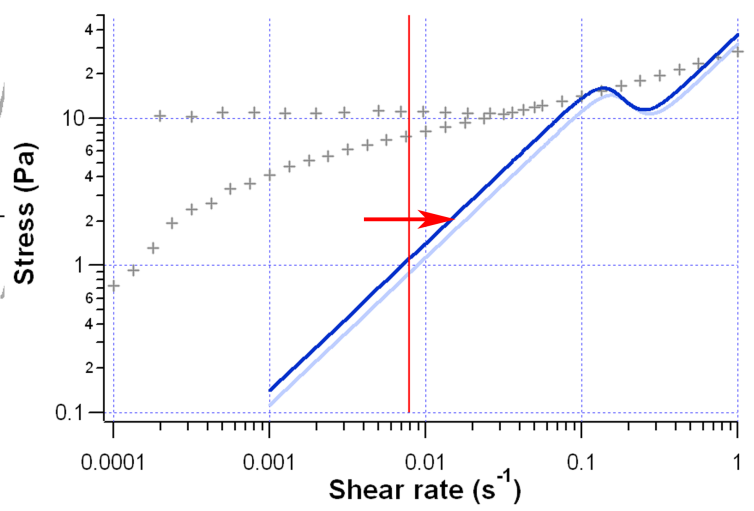

Figure 8.6: A continuation of Fig. 8.5 shows the active flow curve changing as the shear rate switches sign. 


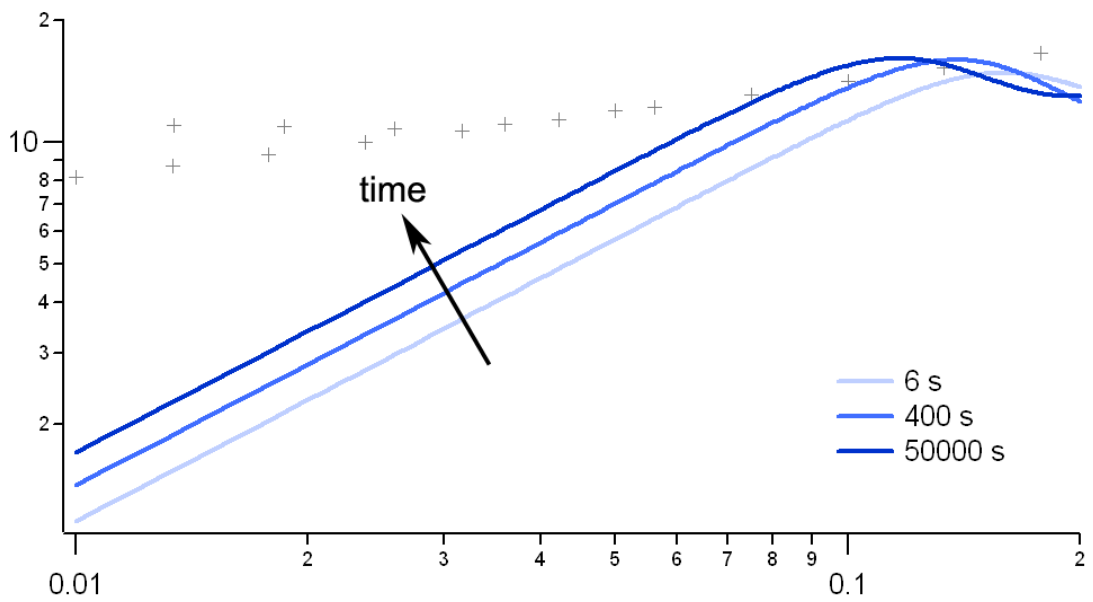

Figure 8.7: The evolution of the Cross model fitting to the upward rate sweep of the LAOS data where the oscillatory shear is applied at a frequency of $1 \mathrm{rad} \mathrm{s}^{-1}$ with an amplitude of $\gamma_{0}=20 \%$.

Figures 8.5 and 8.6 both show a large discrepancy between the 'oscillatory flow curve' in solid lines and the 'steady-shear flow curve' shown with the discrete + symbols. The use of the symbols and lines make clear the difference in the two methods used to obtain them: the steady-shear curve was measured at discrete shear rates with the pseudo-steady- and steady-state stresses plotted as ' + 's and the lines indicate a continuous sweep over all rates $\dot{\gamma} \leq \pm \dot{\gamma}_{\max }$. The discrepancy is interpreted with the visualisation of the startup stress as a function of time, shown in Beris et al. [126] and Rogers et al. [127]. In these step-rate tests, the stress takes the order of $10 s$ to reach the steady values shown by the + points displayed in Figs. 8.5 and 8.6. In the oscillatory tests, each shear rate is passed on the way to a higher/lower shear rate with residence times that are much smaller than the $10 s$ required to reach even the pseudo-steady state. Thus, the oscillatory tests allow a glimpse of the evolution of the short-time stress response with higher frequency tests probing the shorter times and lower frequency tests approximating the steady-shear case. The evolution of the Cross model fits to the LAOS data $\left(\omega_{0}=1 \mathrm{rad} \mathrm{s}^{-1}, \gamma_{0}=20 \%\right)$ is illustrated in Fig. 8.7. While the residence time at each particular rate is infinitely small, the evolution of the form of the oscillatory flow curve towards that of the steady-shear flow curve is obvious, though not complete. The oscillatory curve measured at $1 \mathrm{rad} \mathrm{s}^{-1}, \gamma_{0}=20 \%$ does not match the steady-shear curve even after $50000 s$ of shearing.

We also show the complete figure obtained via this method for the data of Fig. 8.2 
in Fig. 8.8.

The data of Fig. 8.8 show the effectiveness of this method for capturing the essential features of the experimental data that the methods of Ewoldt et al. and Klein et al. fail to. The current method also highlights the asymmetry in the data; an excellent fit is obtained for positive stresses but some discrepancies remain for negative stresses. This asymmetry suggests that there exists a preferred direction of shear which may be a consequence of the preshear protocol, an effect that has previously been seen in the non-zero stress at short times in shear startup experiments (see Fig. 5.2 and Fig. 5.3(a)) as well as an asymmetry in stress relaxation tests.

It is worth making a further comment about the experimental results displayed in this chapter. The LAOS data suggest that at very short times, the flow curve of this material displays a region of unstable negative slope that evolves into a stable branch of positive slope at long times. This material is not alone in displaying this behaviour. Chopra et al. in their investigation of the partially miscible polymer blend poly(styrene-co-maleic anhydride)/poly(methyl methacrylate) [128], saw similar behaviour suggesting a more universal behaviour.

This part of the work is concluded by showing fits for other strain amplitudes at a frequency of $1 \mathrm{rads}^{-1}$ in Fig. 8.9 and a few fits to data at a frequency of $100 \mathrm{rads}^{-1}$ in Fig. 8.10. The parameters used to obtain the Cross model fits are displayed in table 8.1. Note the fit is poorer at higher strain amplitudes and frequencies due to the approximation (that the oscillatory data can be modelled using a steady-shear approach) being less valid. Unfortunately, due to a lack of data, no conclusions can be drawn on the range of experimental variables over which the approximation is valid.

Table 8.1: Cross model parameters

\begin{tabular}{cccc}
\hline$\omega\left(\mathrm{rad} \mathrm{s}^{-1}\right)$ & $\gamma_{0}(\%)$ & parameters (upward sweep) & parameters (downward sweep) \\
& & {$\left[\eta_{\infty}(\right.$ Pas $), \eta_{0}($ Pas $\left.), \alpha, n\right]$} & {$\left[\eta_{\infty}(\right.$ Pas $), \eta_{0}($ Pas $\left.), \alpha, n\right]$} \\
\hline \hline 1 & 10 & $200,10,9.2,8$ & $170,70,8.6,4$ \\
1 & 20 & $141,36,6,6$ & $113,29,5.4,6$ \\
1 & 100 & $64,19.8,1.8,4$ & $40,22,1.8,4$ \\
100 & 25 & $197,20,3.8,8$ & $147,90,3.8,8$ \\
100 & 50 & $185,1,2.02,8$ & $105,75,2.02,8$ \\
\hline
\end{tabular}



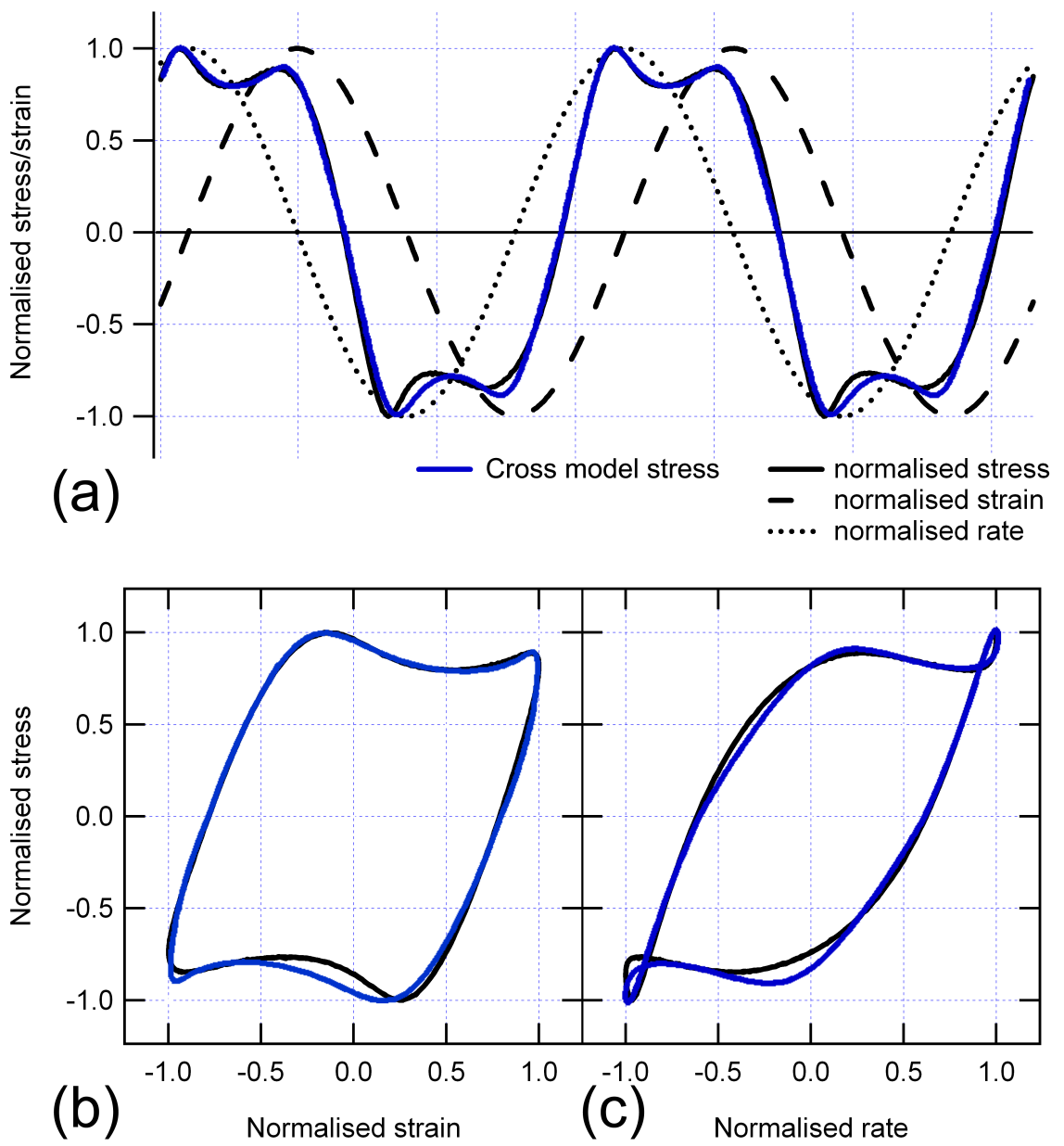

Figure 8.8: The full fit to the data of Fig. 8.2 via the method described in the text. The phenomenology of the stress decrease is now well followed and the asymmetry in the experimental data is highlighted. The asymmetry suggests a preferred direction of shear that may be a layover from the preshear protocol. 


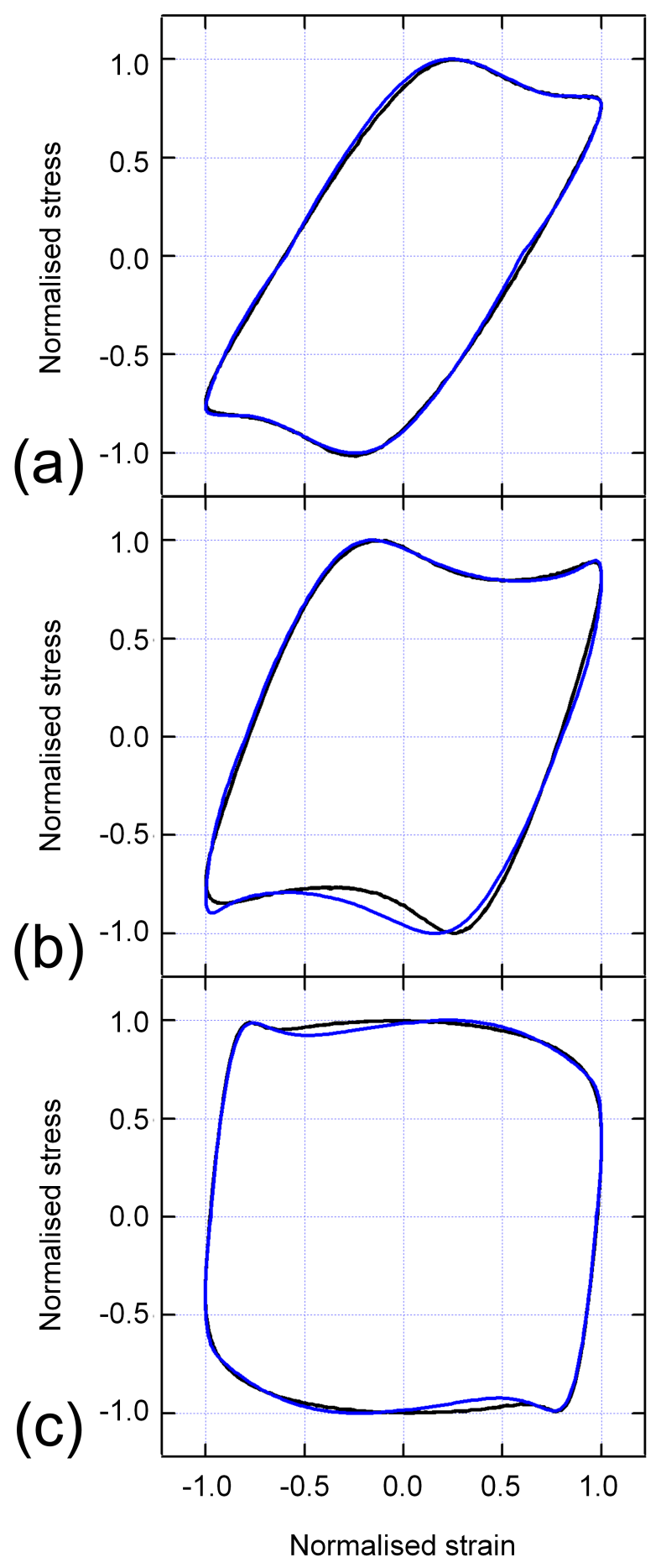

Figure 8.9: Stress responses in black with fits in blue by the method described in the text for oscillatory strains of $\omega=1 \mathrm{rad} \mathrm{s}^{-1}, \gamma_{0}=10 \%$ (a), $20 \%$ (b) and $100 \%$ (c) 


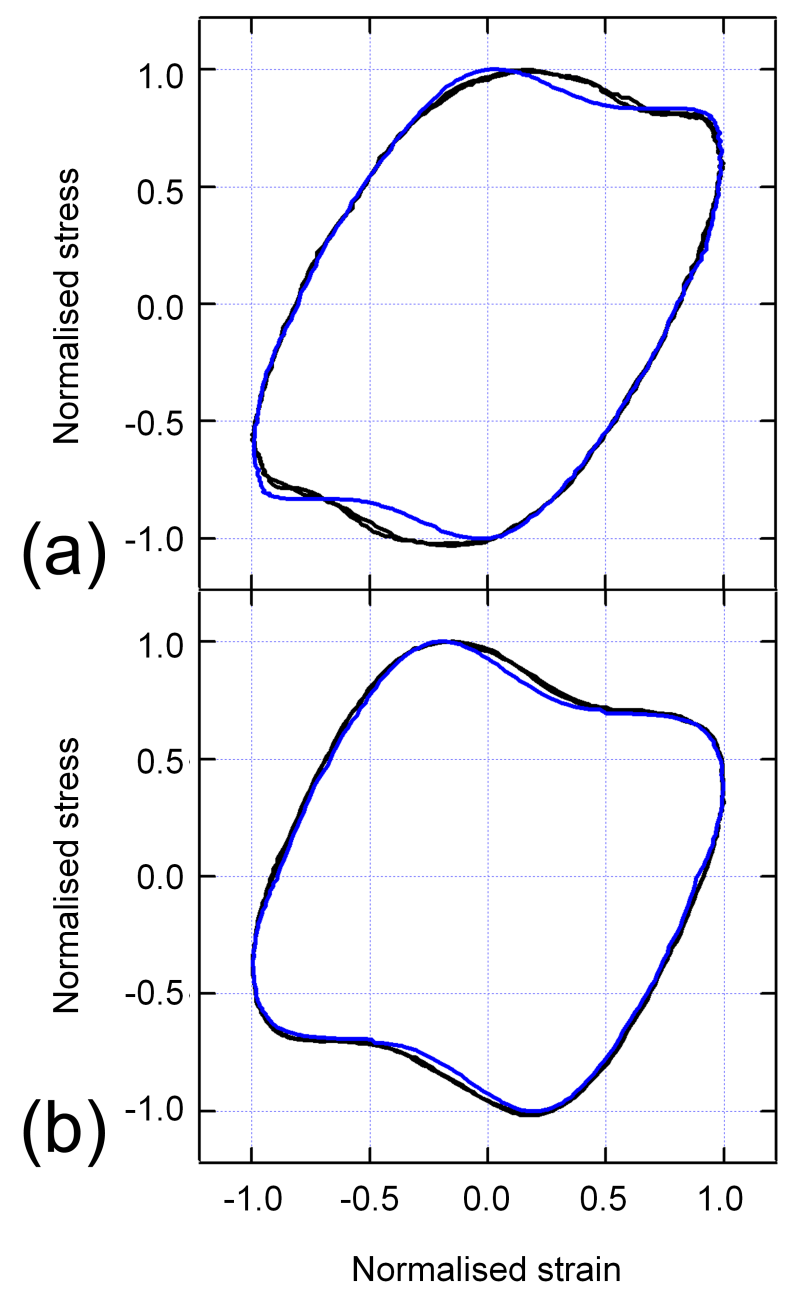

Figure 8.10: Stress data $\omega=100 \mathrm{rads}^{1}$, (a) $\gamma_{0}=25 \%$, (b) $\gamma_{0}=50 \%$ in black with fits in blue by the method described in the text. 


\subsection{Parametrization}

Having successfully mapped both the increasing and decreasing shear-rate branches of the traversed flow curve, it remains to make a few comments on a simple parametrization under the current model. The Cross model involves four parameters $\left(\eta_{0}, \eta_{\infty}, \alpha\right.$ and $n$ ) which do not lend themselves readily to a single parameter from which something can be gathered regarding the nonlinearity of a response. It can be stated that for shear rates $\dot{\gamma}<<\alpha^{-\frac{1}{n}}$, the response will be linear and able to be modelled by a viscosity $\eta_{0}$ and for shear rates $\dot{\gamma} \sim \alpha^{-\frac{1}{n}}$, the response will be nonlinear, but using this formality requires prior knowledge of the values of these parameters. Instead, we look to the energy dissipated per unit volume per cycle, $E_{d}$, which is defined as

$$
E_{d}=\oint \sigma d \gamma=\oint \sigma(t) \dot{\gamma}(t) d t
$$

$E_{d}$ is thus the area enclosed by a Lissajous curve of (non-normalised) stress, $\sigma$, as a function of (non-normalised) strain, $\gamma$. The ratio of energy dissipated by the sample under specific experimental conditions, $E_{\exp }$ to the energy dissipated by a Newtonian fluid whose viscosity matches that of the test substance at small strains, $E_{\text {Newtonian }}$ gives a 'linear ratio', $\frac{E_{\text {exp }}}{E_{\text {Newtonian }}}$. The linear ratio is identically 1 for a completely linear response, less than 1 for a response that includes a stiffening and greater than 1 for a response that includes a softening. The Newtonian response required can be found by fitting a sinusoid to the small-strain stress response. The amplitude of the fitted sinusoid divided by the strain amplitude gives an approximation to $\eta_{0}$, the zero-shear viscosity. In the Cross model the two limiting cases are: for small strains the ratio is identically 1 as the stress response is Newtonian with a viscosity $\eta_{0}$; for large strains the ratio is equal to $\frac{\eta_{\infty}}{\eta_{0}}$, the ratio of limiting viscosities. Figure 8.11 illustrates this procedure carried out on the experimental data of Fig. 8.2. A linear ratio of 0.64 is found, indicating a nonlinear response, as expected.

\subsection{Symmetry of response}

The methods of Ewoldt et al. Klein et al. the Cross model fitting described above and the traditional method of assigning dynamic moduli assume a symmetric stress response for all oscillatory-strain experiments. In the technical sense, using Conway's orbifold notation, these methods assume the stress response is an $\infty x$ (step) frieze 
(a)
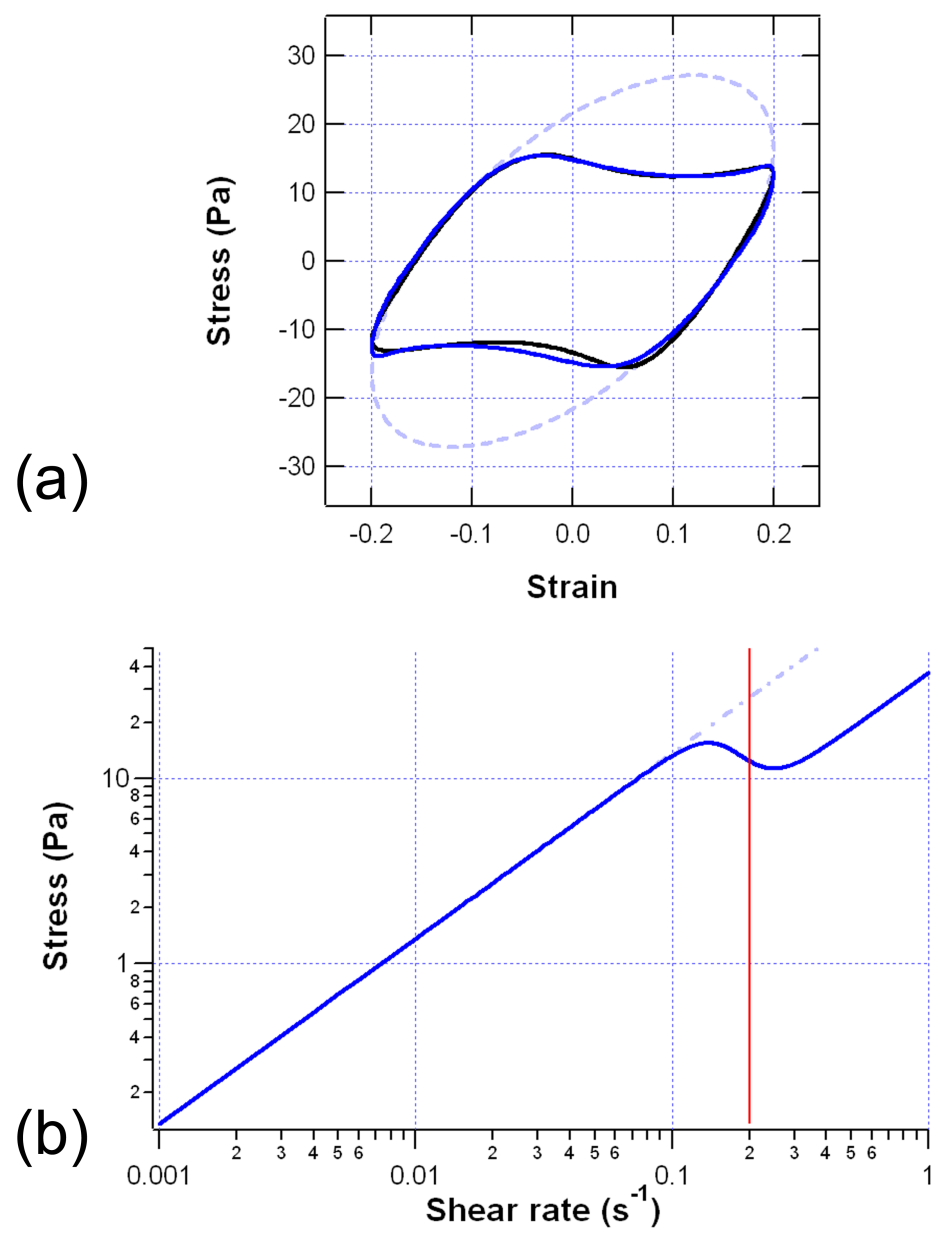

Figure 8.11: (a) A sinusoid (light blue broken line) is fitted to the small-strain stress response of the sample. The viscosity of the sinusoidal response is $\eta_{0}$, the zero-shear viscosity, which is a continuation of the lower branch of the flow curve as indicated in (b). The ratio of areas swept by the Lissajous curves of the experimental result to the Newtonian case gives a linear ratio of 0.64 . The maximum shear rate reached is indicated by the red line. 
group. That is, an assumption is made that the stress response from one direction of strain is identical to the response from the other. The results of the Cross model fitting method described above make clear that this is not necessarily the case and that the data is actually more closely related to an $\infty \infty$ (hop) frieze with an entire stress-period being the repeating unit.

It is of interest to quantify by how much the positive and negative lobes differ from each other and how this changes over the course of an experiment. The most obvious step in such an analysis may be to look at the extrema and amplitude of the stress response as a function of time. The extrema are defined as being the most positive and most negative stress values achieved each period and the amplitude is the absolute difference between the two. Such information is shown in Figs. 8.12 - 8.14 for $1 \mathrm{rad} \mathrm{s}^{-1}$, $\gamma_{0}=0.1,1,10,20,100$ and $500 \%$.

The maximum and minimum stress as a function of time is shown on the left of Figs. 8.12 - 8.14 and shows an asymmetric evolution of the stress extrema in each case. The amplitude is plotted as a function of time on the right of Figs. 8.12 - 8.14 and shows a maximum change of more than $2 \mathrm{~Pa}$ over the course of $10^{4} \mathrm{~s}$ when $\gamma_{0}=10 \%$. The data of Figs. 8.12 and 8.13 indicate the presence of oscillations in the stress responses to oscillatory strains of amplitudes $\gamma_{0}=0.1,1$ and $10 \%$. The oscillations share a common period of $\sim 360 \mathrm{~s}$. These oscillations are associated with the temperature control cycle of the air conditioner unit over the course of the experiments. Such oscillations are visible throughout the data presented in this chapter and are not thought to be associated with some previously unknown physics of the material. Rather, they represent the response to unknown variations in temperature.

The data of Figs. 8.12 - 8.14 show a lack of symmetry in terms of the stress extrema and Fig. 8.8 shows a lack of symmetry in terms of the shape of the stress response. We can therefore state that the stress response, while assumed to be an $\infty x$ (step) frieze group, though more closely resembling an $\infty \infty$ (hop) frieze, is actually neither. The data do not constitute a frieze group at all for the experimental times investigated here. Any analysis technique that assumes otherwise necessarily discards some information and risks obtaining misleading or incomplete conclusions.

In order to more exhaustively analyse the current data sets, we begin by quantifying how close to a frieze group the responses are; that is, the symmetry is quantified. To quantify the symmetry, the amount of energy dissipated in each lobe is calculated via equation 8.2. The energy dissipated in the positive-stress lobe is divided by the energy 

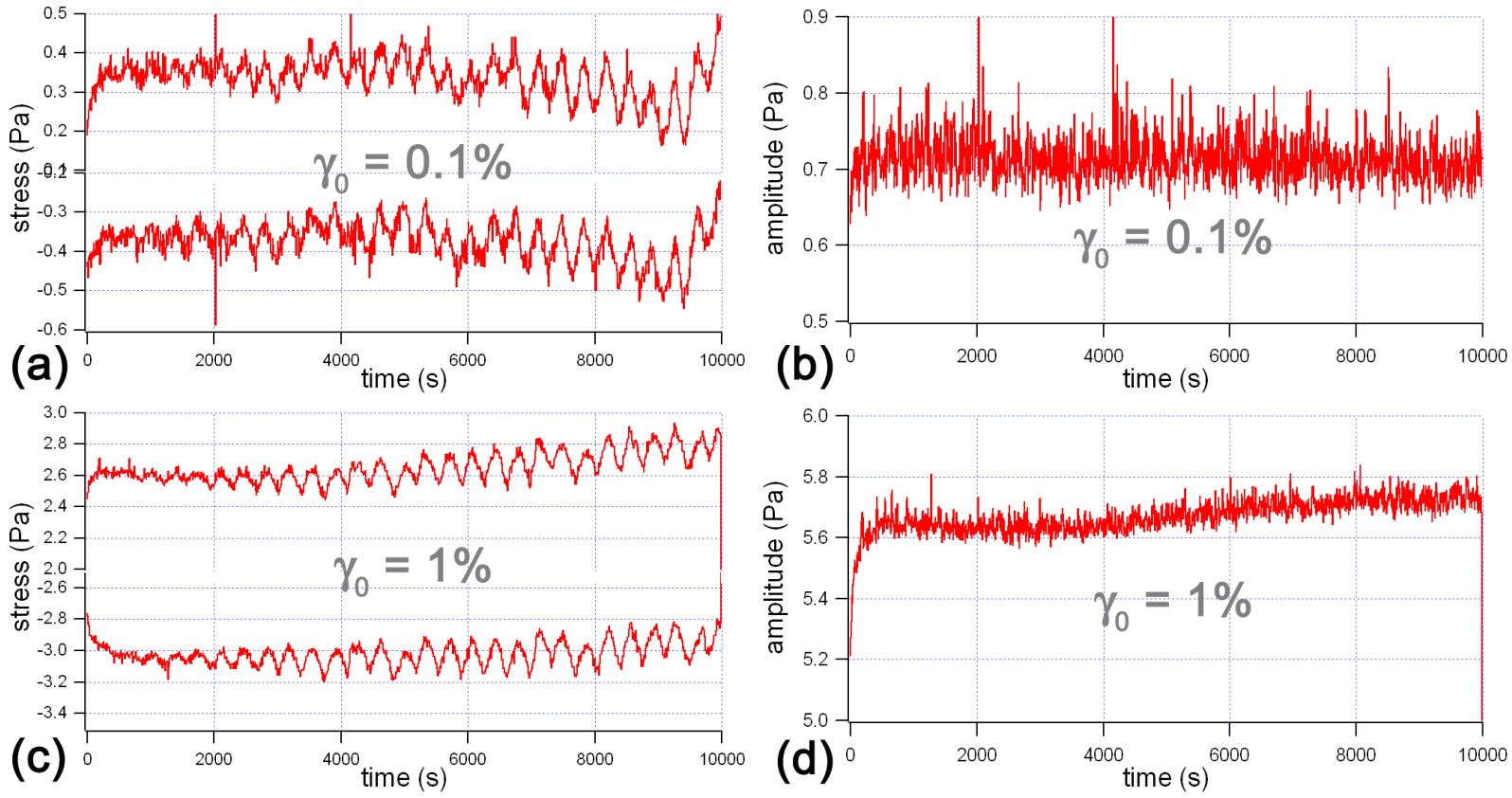

Figure 8.12: (a) The maximum (top) and minimum (bottom) stresses and (b) stress amplitude as a function of time for an oscillatory strain of $\omega=1 \mathrm{rads}^{-1}, \gamma_{0}=0.1 \%$. (c) and (d) show corresponding data for $\gamma_{0}=1 \%$. The oscillations present are due to temperature variations and the response cycle of the air conditioning unit. The amplitude of these oscillations in the stress response when $\gamma_{0}=1 \%$ increases by $0.1 P a$ between 4000 and $8000 \mathrm{~s}$. 

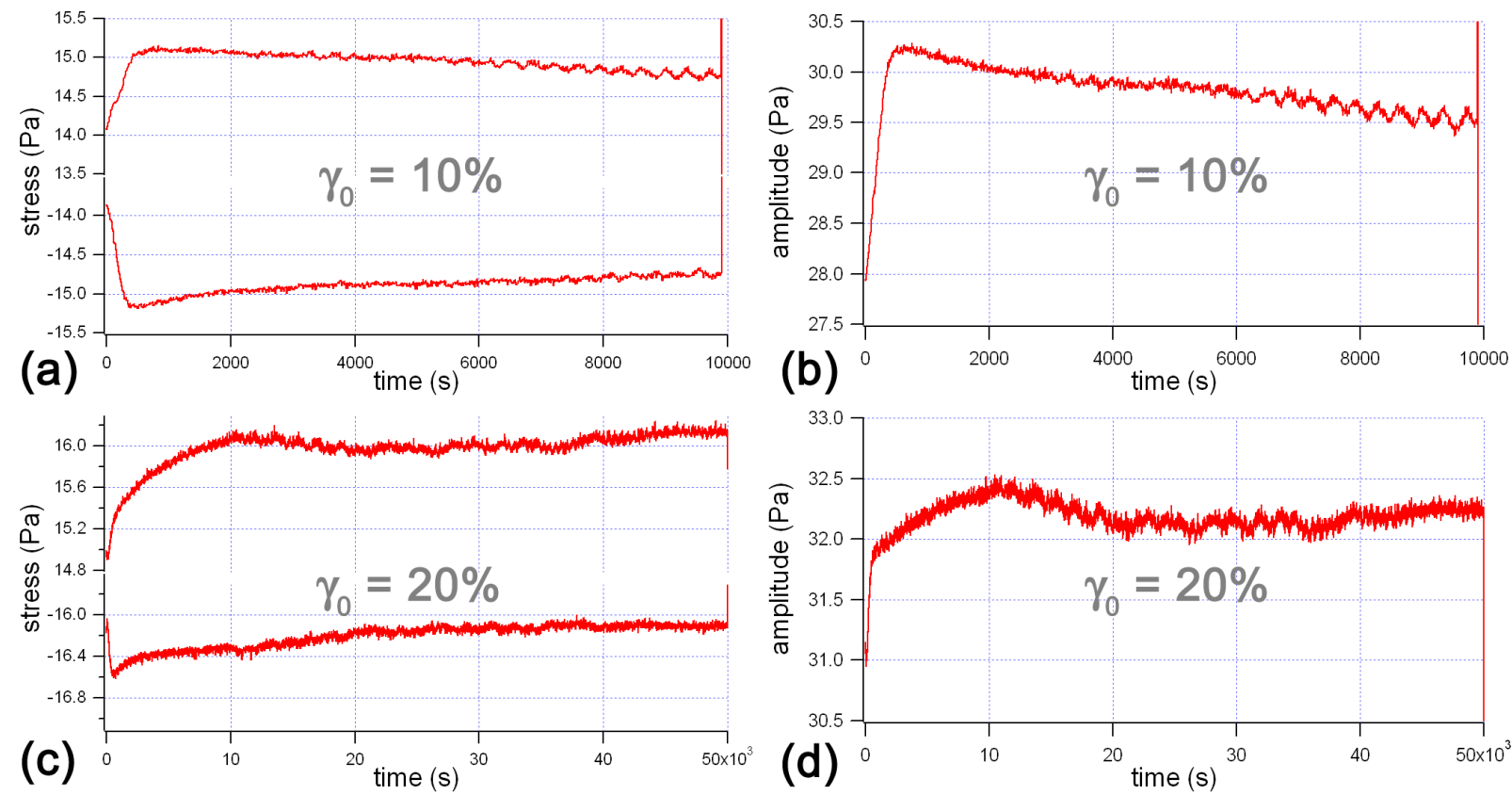

Figure 8.13: (a) The maximum (top) and minimum (bottom) stresses and (b) stress amplitude as a function of time for an oscillatory strain of $\omega=1 \mathrm{rads}^{-1}, \gamma_{0}=10 \%$. (c) and (d) show corresponding data for $\gamma_{0}=20 \%$. Note the difference in time scale for (c) and (d). Under both strain conditions, the amplitude of the stress response rises sharply initially. (b) shows the amplitude of the stress response to a strain amplitude of $10 \%$ beginning to oscillate after $\sim 6000 s$ and the amplitude of the oscillation increasing up to the end of the experiment, reflecting the response of the air conditioner to the changing temperature in the laboratory during the experiment. 

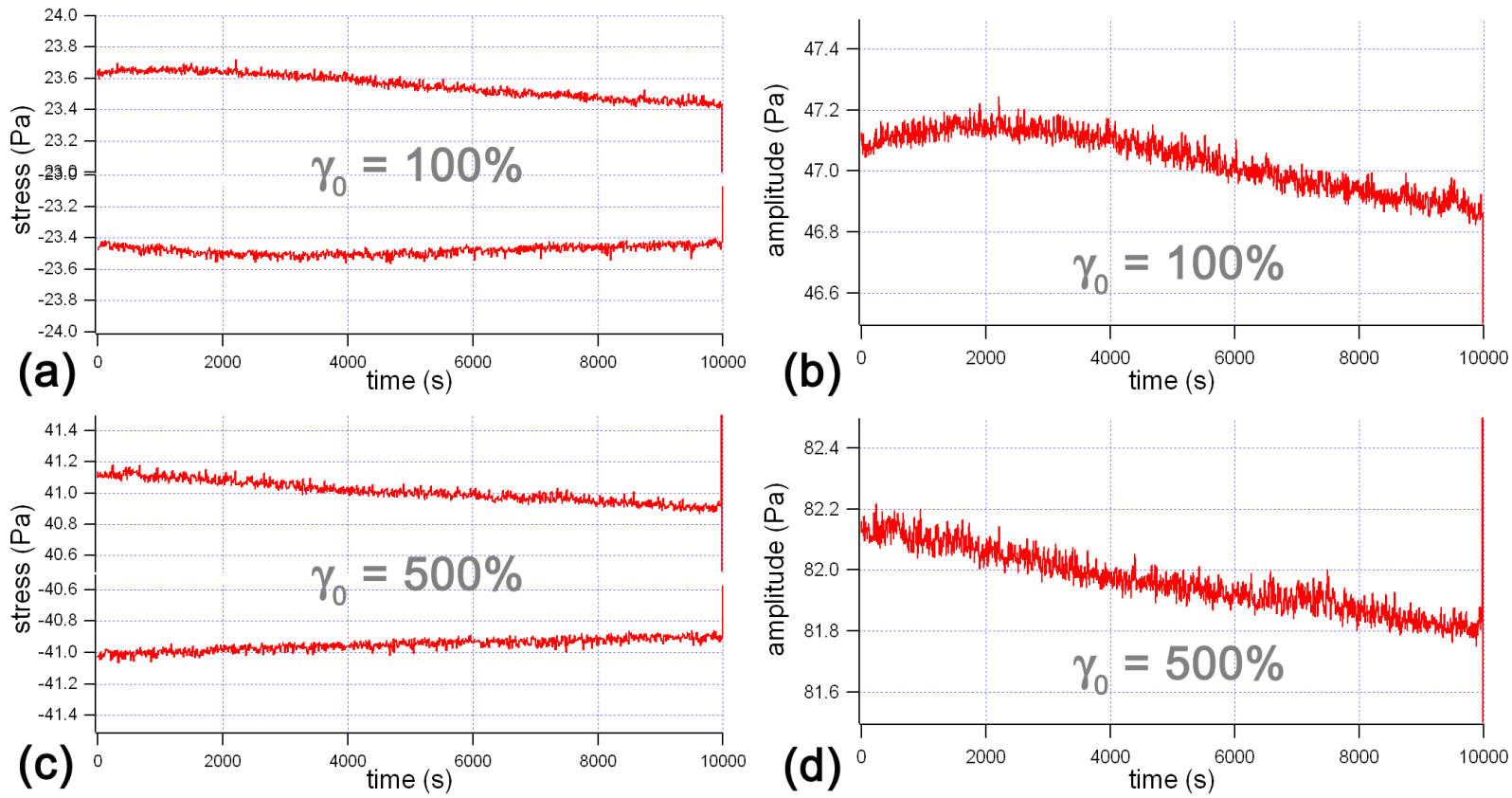

Figure 8.14: (a) The maximum (top) and minimum (bottom) stresses and (b) stress amplitude as a function of time for an oscillatory strain of $\omega=1 \mathrm{rads}^{-1}, \gamma_{0}=100 \%$. (c) and (d) show corresponding data for $\gamma_{0}=500 \%$. The amplitude of the stress response to a strain amplitude of $100 \%$ (b) shows a maximum amplitude is reached around $2000 s$, after which the amplitude steadily decreases for the remainder of the experiment. The amplitude of the stress response to oscillatory strain of $\gamma_{0}=500 \%$ decreases linearly over the entire experimental time of $10^{4} \mathrm{~s}$, dropping by $\sim 0.4 \mathrm{~Pa}$. 
dissipated in the negative-stress lobe so that a dimensionless number is achieved that represents the contribution to the total energy dissipation from each lobe. A value of this energy-dissipation ratio of 1 corresponds to equal energy dissipated in each direction while a value greater than 1 means more energy is dissipated in the positivestress direction and a value less than 1 means more energy is dissipated in the negativestress direction. It is useful to note that an increase in energy dissipation corresponds to a more liquid-like response and so the energy-dissipation ratio gives an indication of a preferred direction of flow. As a reference for the rest of this section, the dynamic moduli as functions of strain amplitude are shown in Fig. 8.15.

The evolution of the energy-dissipation ratio for various strain amplitudes at a frequency of $1 \mathrm{rad} \mathrm{s}^{-1}$ is shown in Figs. 8.16 - 8.20. The data of Fig. 8.15 suggest a linear behaviour for strain amplitudes $\gamma_{0} \leq 2 \%$. The response of materials in this region is normally considered to be well characterised and so it may be assumed that the evolution of the correlation magnitude would be a random scatter about 1 . The data of Fig. 8.16 supports this assumption for shear conditions far from the traditionallydefined crossover region. The data of Fig. 8.17 suggests that the linear region ends before any change in the dynamic moduli is detected. The evolution of the energydissipation ratio when the strain amplitude is $1 \%$, Fig. 8.17, shows definite structure, with fluctuations of period $\sim 360 s$ and increasing amplitude being the most obvious feature. It is noted again that these oscillations are due to the response cycle of the air conditioning unit used to control the temperature in the laboratory. Underlying the periodic oscillations is an initial trend away from 1 for the first $3000 \mathrm{~s}$. At a time of $\sim 3000 s$ after preshearing, the energy-dissipation ratio turns and for the remainder of the experimental observation time of $10000 s$ approaches 1 , with the periodic (temperature-related) oscillation increasing in amplitude over the entire experimental time.

When the strain amplitude is increased so that, from Fig. 8.15, the shear-melting regime is probed, the energy-dissipation ratio evolves as shown in Fig. 8.18. In the case where $\gamma_{0}=10 \%$, the air conditioning-related oscillations observed in Fig. 8.17 are apparent at times $t>2000 \mathrm{~s}$. This is accounted for by noting the experiment began at $10: 54 \mathrm{am}$ and ended at $1: 34 \mathrm{pm}$, during which the outdoor temperature increased, placing a greater load on the in-lab air-conditioning unit. There is a more dramatic structure observed in the evolution of the energy-dissipation for both the $\gamma_{0}=10 \%$ and $\gamma_{0}=20 \%$ cases. This structure takes the form of non-periodic oscillations. In both 


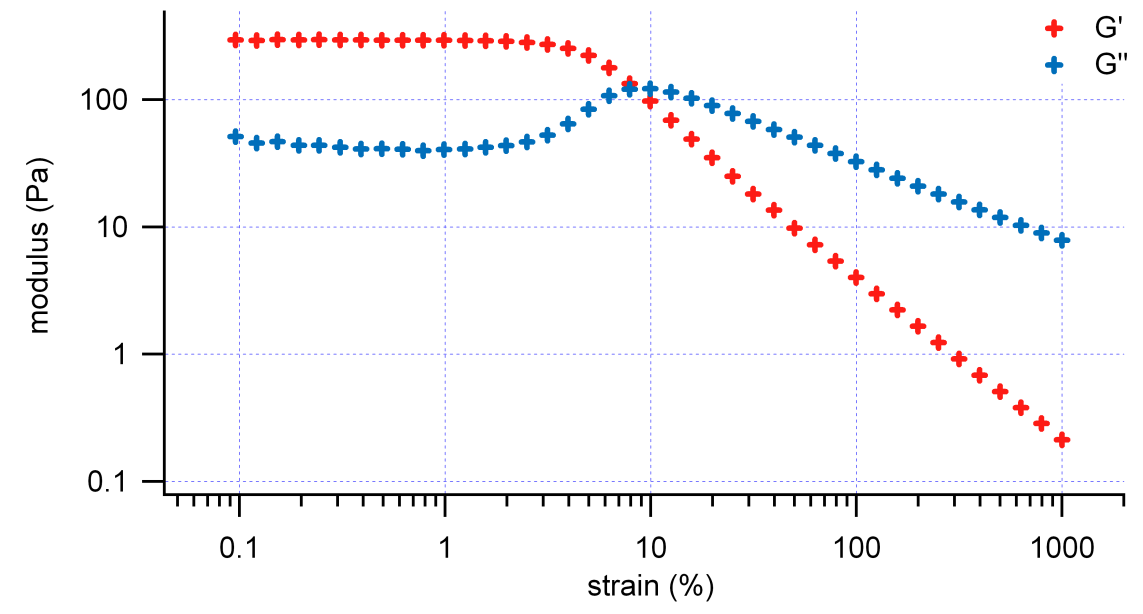

Figure 8.15: Dynamic moduli as functions of strain amplitude for a frequency of $1 \mathrm{rads}^{-1}$.

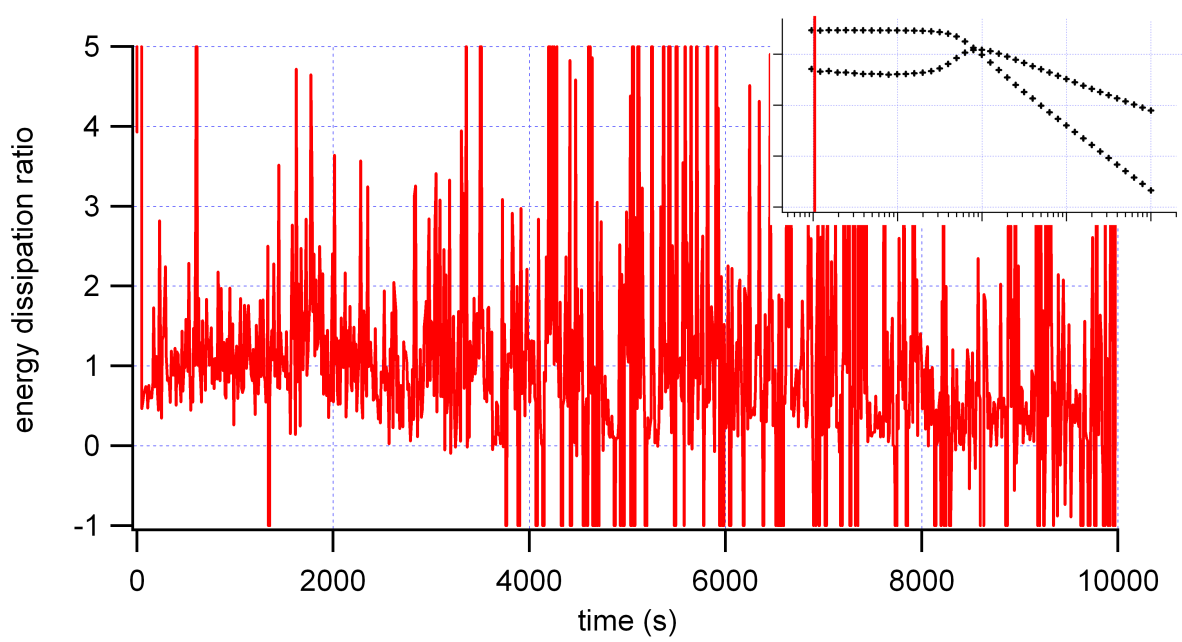

Figure 8.16: Energy-dissipation ratio as a function of time for a strain amplitude of $\gamma_{0}=0.1 \%$. The noise in the data combined with the extremely small areas involved make for a noisy energy-dissipation ratio. Inset: Inset: Dynamic moduli as functions of strain amplitude showing relative position (red line) of the strain conditions. 


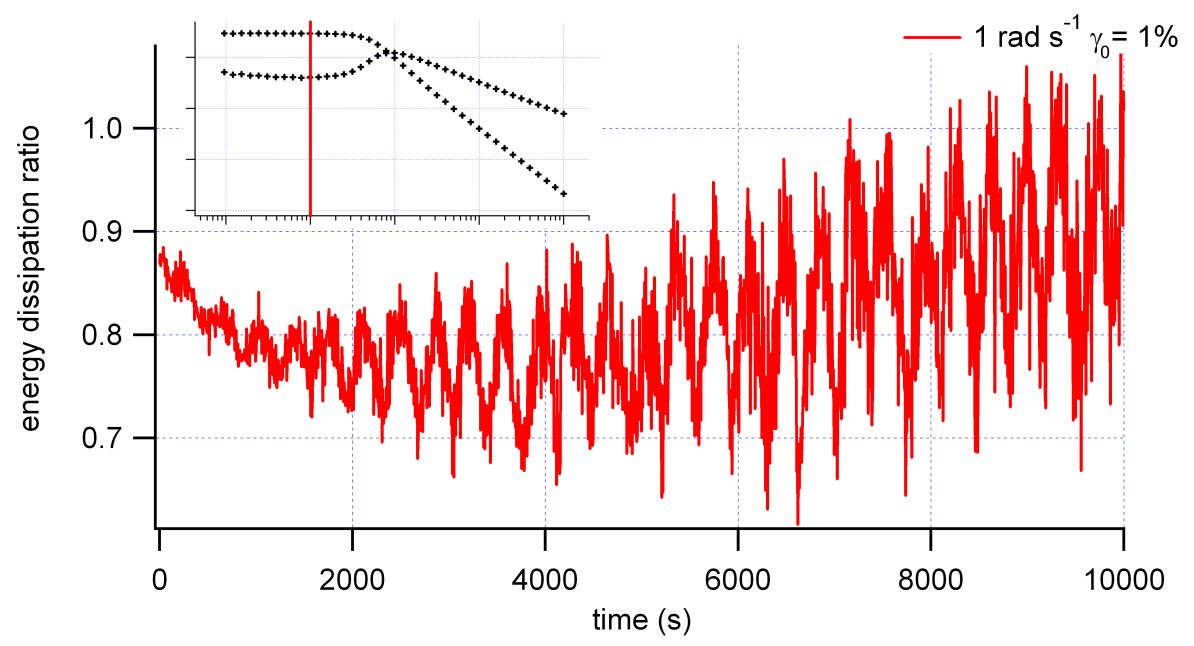

Figure 8.17: Energy-dissipation ratio for a strain amplitude of $\gamma_{0}=1 \%$. As suggested by Fig. 8.12(c), the ratio is susceptible to the $\sim 360 s$ oscillations of the air conditioning unit, while slowly approaching 1. Inset: Dynamic moduli as functions of strain amplitude showing relative position of the strain conditions.

cases, the first feature is a decrease in the energy-dissipation ratio. In the $\gamma_{0}=10 \%$ case, this 'dip' is replaced by a longer-period oscillation that takes the ratio above 1 for $\sim 5000 \mathrm{~s}$ before returning below 1 for the remainder of the experiment. In the $\gamma_{0}=20 \%$ case, the initial oscillation gives way to a steady approach toward 1 after $\sim 500 s$.

The evolution of the energy-dissipation ratio for $\omega=1 \mathrm{rads}^{-1}, \gamma_{0}=20 \%, t \leq$ $50000 s$ is shown in Fig. 8.19. The behaviour of the energy-dissipation ratio for times $10000 s \leq t \leq 50000 s$ differs from that of earlier times in that there is a stepwise approach towards 1 , suggesting stepwise, discrete physics is responsible.

The evolution of the energy-dissipation ratio in the shear-thinning regime is displayed in Fig. 8.20. At such large strain amplitudes the energy-dissipation ratio tends toward 1 in a stepwise manner similar to the long-time response when $\gamma_{0}=20 \%$. The larger the amplitude the more symmetric the initial response is and the slower the symmetry limit is approached.

The data displayed in Figs. 8.16, 8.17, 8.18 and 8.19 suggest two separate regimes with a wide transition between them similar to those discernable in Fig. 8.15. In the linear regime at strain amplitudes far below the crossing of the dynamic moduli, the evolution of the correlation magnitude is characterised by random scatter about 1 (Fig. 8.16). Far into the shear-thinning regime, the energy-dissipation ratio approaches 


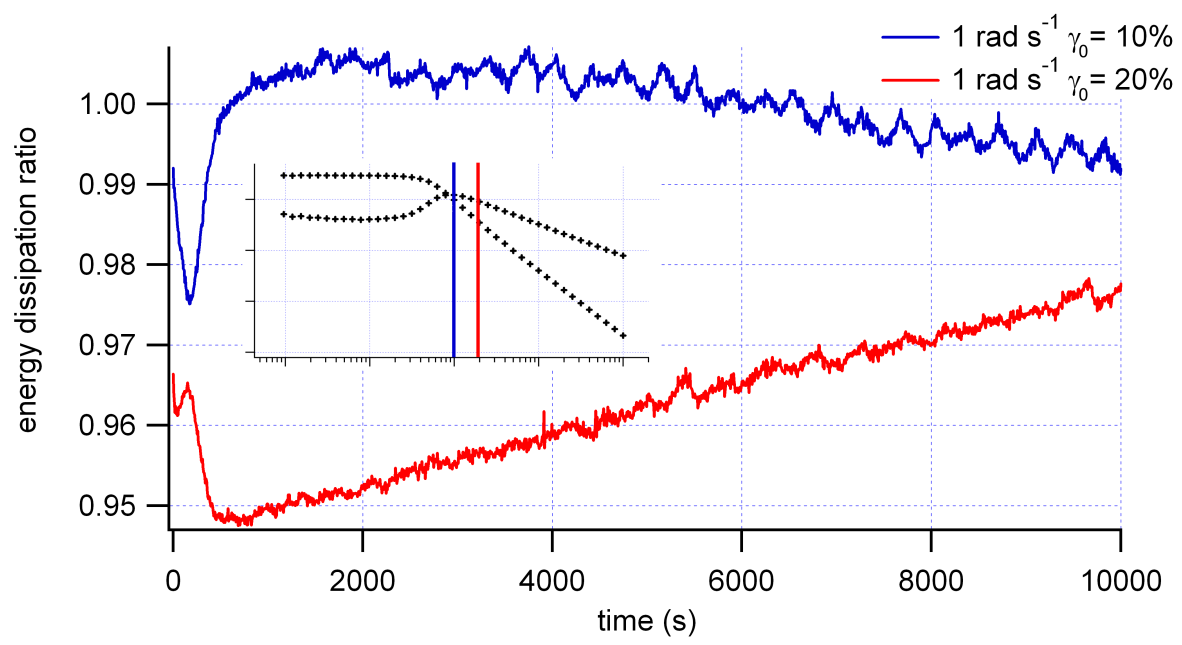

Figure 8.18: Energy-dissipation ratios for strain amplitudes of $\gamma_{0}=10 \%$ (blue) and $\gamma_{0}=20 \%$ (red) as functions of time. The steady air conditioning-related oscillations shown in Fig. 8.13(a) and (b) are seen in the $\gamma_{0}=10 \%$ line. Inset: Dynamic moduli as functions of strain amplitude showing relative positions of the strain conditions.

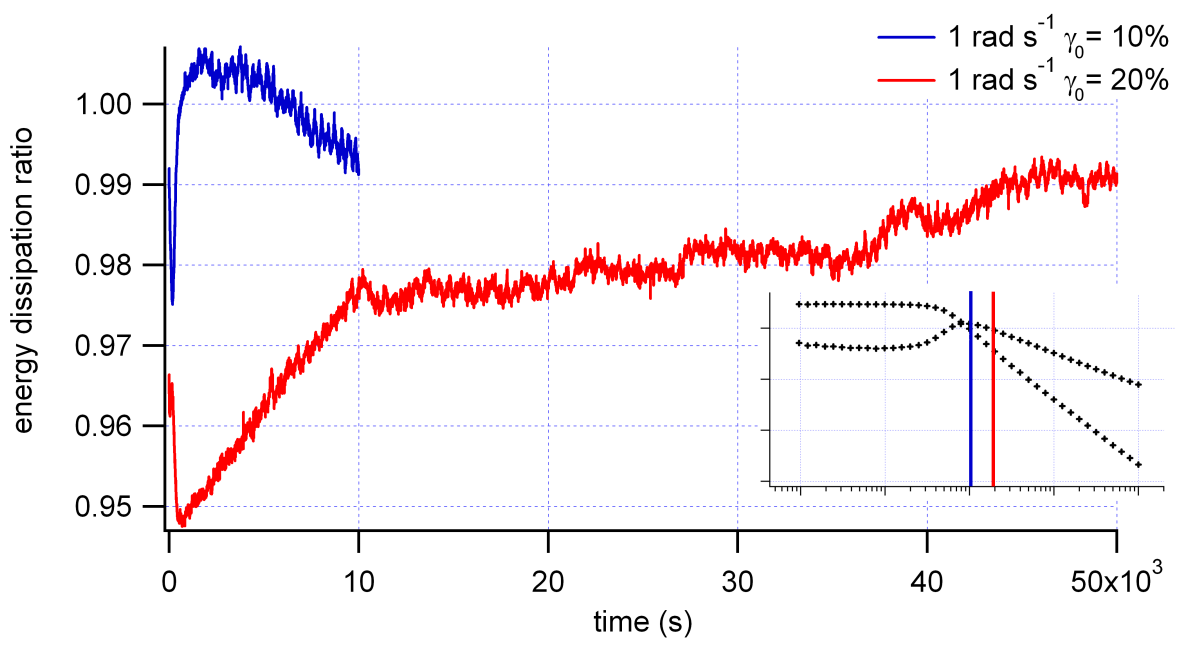

Figure 8.19: A temporally extended version of Fig. 8.18 showing the evolution of the $20 \%$ case up to $5 \times 10^{4} \mathrm{~s}$. The smooth approach towards 1 that was characteristic of Fig. 8.18 has given way to a discrete step-wise approach. 


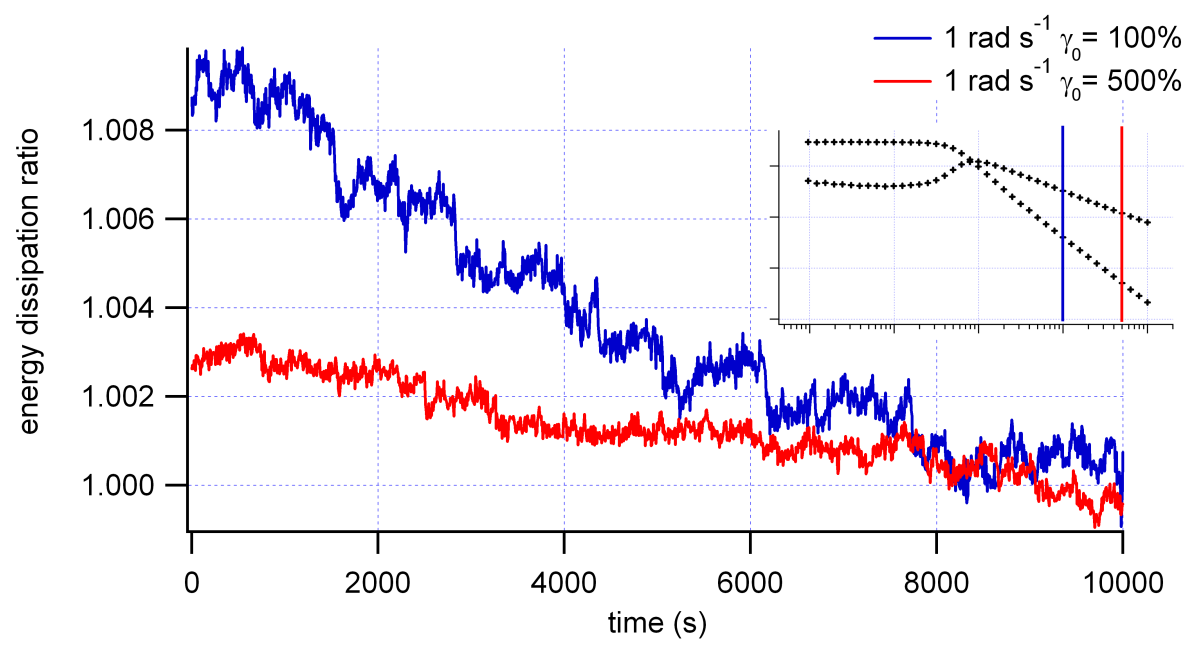

Figure 8.20: Energy-dissipation ratios for strain amplitudes of $\gamma_{0}=100$ (blue) and $500 \%$ (red). A discrete step-wise approach towards 1 is observed throughout the experiment in both cases.

1 in a stepwise manner. The farther into the shear-thinning regime a set of experimental conditions are, the more symmetric the initial response is and the slower the approach towards 1 . These two extreme behaviours correspond to the classical linear and shear-thinning regimes well. In between is a large transition regime that shows combinations of both extreme cases.

At the low-strain amplitude end of the transition region, see Fig. 8.17 for instance, the energy-dissipation ratio ultimately tends to 1 (ignoring the oscillations related to the temperature variations due to the cycle of the temperature control unit of the air conditioning unit) suggesting the physics occurring is different from that of the true linear region, displayed in Fig. 8.16. At long times at the high-strain amplitude end of the transition region (as displayed in Fig. 8.19), the energy-dissipation ratio evolves as if the sample was deep in the shear-thinning regime, that is, asymptotically approaching 1 in a stepwise, discrete manner. At a strain amplitude that correlates to the crossover of the dynamic moduli the energy-dissipation ratio is unstable and oscillates about, but never asymptotically approach 1 . This analysis technique suggests that the linear regime does not extend to strain amplitudes as large as the dynamic moduli suggest.

While the energy-dissipation ratio provides a useful way of quantifying the symmetry, it can be equal to 1 without the two lobes being identical shapes. To avoid any possible misinterpretation, a second parameter is calculated simultaneously that al- 
lows for differences in shape to be accounted for. An ideal parameter for such a task is the path-length difference. We define the path-length difference to be the difference in the path length between the positive lobe and the negative lobe. This difference is normalised by division by the path-length of the positive lobe such that the difference is expressed as a percentage of the path length of the positive lobe. The path length of a lobe is defined by the relation

$$
\text { path length }=\int_{L} \mathrm{ds}
$$

where the subscript L means the integral is over a lobe and ds is an elementary arc length defined for a discrete data set $\left(\tau_{i}, \gamma_{i}\right)$ as

$$
\mathrm{d} \mathbf{s}=\sqrt{\left(\tau_{i+1}-\tau_{i}\right)^{2}+\left(\gamma_{i+1}-\gamma_{i}\right)^{2}} .
$$

A perfectly symmetric response in the (energy-dissipation ratio)-(path-length difference) plane corresponds to an energy-dissipation ratio of 1 and a path-length difference of 0 . An energy-dissipation ratio of 1 and a positive path-length difference is indicative of a more curved positive lobe while an energy-dissipation ratio of 1 with a negative path-length difference is indicative of a more curved negative lobe.

The extra information gathered by following the trace in (energy-dissipation ratio)(path-length difference) space only seems to be valuable in the shear melting regime. In the linear and shear-thinning regimes, the path-length difference is characterised by nearly random scatter about 0 . The trace through (energy-dissipation ratio)-(pathlength difference) space is depicted in Fig. 8.21 for an oscillatory shear of $\omega=1 \mathrm{rad} \mathrm{s} \mathrm{s}^{-1}, \gamma_{0}=$ 10 (a) and $20 \%$ (b). The type of asymmetry in each case changes as a function of time, as indicated by the evolution into different quadrants of (energy-dissipation ratio)(path-length difference) space. As a comparison, the trace through (energy-dissipation ratio)-(path-length difference) space is also shown in Fig. 8.22 for an oscillatory shear in the shear-thinning regime of $\gamma_{0}=100 \%$ showing the asymmetry reduces throughout the entire experiment.

\subsection{Total energy dissipation}

The previous section examined the evolution of the symmetry of the response with respect to a number of 'symmetry parameters'. Each parameter showed that the response to LAOS, and indeed SAOS, cannot be considered a frieze group, which would 

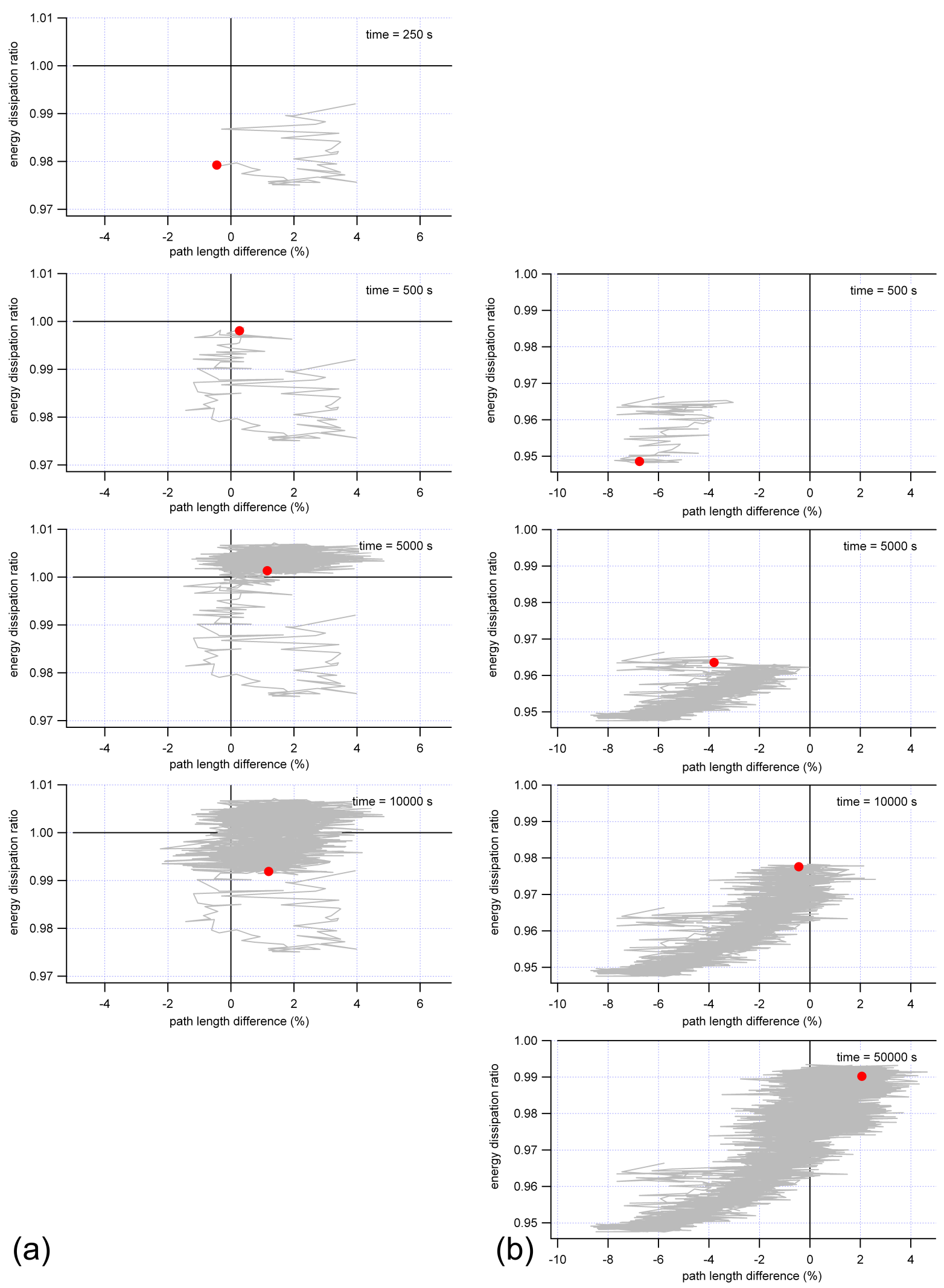

(a)

(b)

Figure 8.21: Following the trace through (energy-dissipation ratio)-(path-length difference) space indicates the lack of symmetry in the response to oscillatory strains of $\omega=1 \mathrm{rad} \mathrm{s}{ }^{-1}, \gamma_{0}=10 \%$ (a) and $20 \%$ (b). The type of asymmetry in each case changes as a function of time, as indicated by the evolution into different quadrants of (energy-dissipation ratio)-(path-length difference) space. 

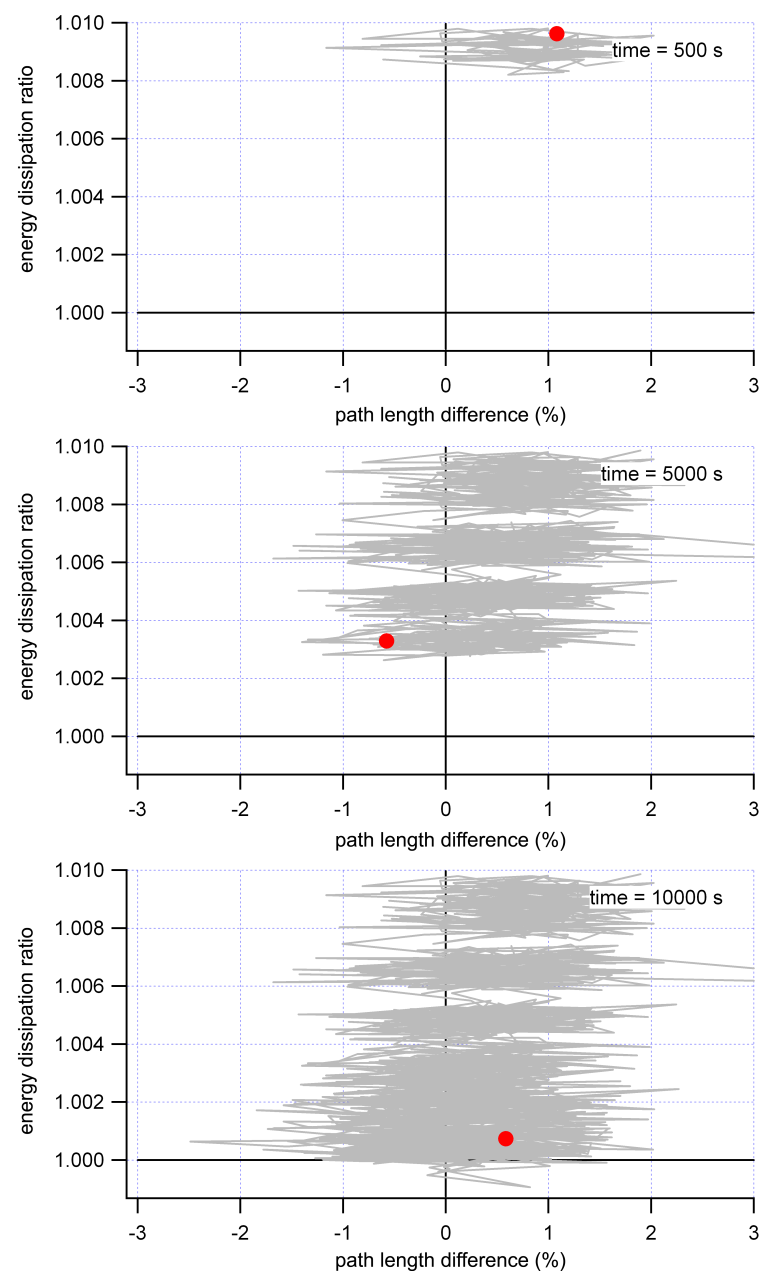

Figure 8.22: The evolution of the LAOS response to a strain of frequency $\omega_{0}=1 \mathrm{rads}^{-1}$ and an amplitude of $\gamma_{0}=100 \%$. In contrast to the data presented in Fig. 8.21, the path-length difference oscillates about $0 \%$ for the entire experiment. The step-wise decreases in the energy-dissipation ratio are seen in this figure as horizontal bands. 
enable the symmetric analysis techniques to be employed with absolute faith in the conclusions drawn from their results. The parameters examined were the stress extrema, taken directly from the raw data, the path-length difference, which gives a measure of curvature differences and the energy-dissipation ratio, which is the relative proportion of energy dissipated in the positive stress lobe to the negative. The combination of the shape of the LAOS response with all these parameters, especially the energy-dissipation ratio, produce compelling evidence of a preferred stress direction and hence a spatial heterogeneity. This evidence will be used to argue the existence of clustering and of rearrangements of clusters. To add further weight to that discussion, this section will present the evolution of total energy dissipated per unit volume per cycle as per equation 8.2.

Before the data is presented we note that if the stress response can be represented as a Fourier series involving the dynamic moduli and their harmonics (as explained in section 2.4.1),

$$
\sigma(t)=\gamma_{0} \sum_{n=1}^{N}\left[G_{n}^{\prime}\left(\omega, \gamma_{0}\right) \sin (n \omega t)+G_{n}^{\prime \prime}\left(\omega, \gamma_{0}\right) \cos (n \omega t)\right]
$$

then equation 8.2 reads

$$
\begin{aligned}
E_{d} & =\oint \sigma d \gamma=\oint \sigma(t) \dot{\gamma}(t) d t \\
& =\gamma_{0}^{2} \omega \int_{0}^{2 \pi / \omega} \sum_{n=1}^{N}\left[G_{n}^{\prime} \sin (n \omega t)+G_{n}^{\prime \prime} \cos (n \omega t)\right] \cos (\omega t) d \mathbf{t} \\
& =\gamma_{0}^{2} \omega G_{1}^{\prime \prime} \int_{0}^{2 \pi / \omega} \cos ^{2}(\omega t) d \mathbf{t} \\
& =\gamma_{0}^{2} \pi G_{1}^{\prime \prime} .
\end{aligned}
$$

That is, the traditionally used loss modulus, $G^{\prime \prime}$ is the energy dissipated per unit volume per cycle scaled by $\gamma_{0}^{2} \pi$. In this chapter, we will use $E_{d}$ preferentially over $G^{\prime \prime}$ and retain the units of $\mathrm{J} \mathrm{m}^{-3}$. In this way, it is hoped a more physical understanding of the processes involved will be provided.

Presented in Figs. 8.23 - 8.27 are the evolutions of the total energy dissipated per unit volume per cycle for an angular frequency of $\omega=1 \mathrm{rad} \mathrm{s}^{-1}$ and strain amplitudes of $\gamma_{0}=1 \%$ (Fig. 8.23), $\gamma_{0}=10 \%$ (Fig. 8.24), $\gamma_{0}=20 \%$ (Fig. 8.25), $\gamma_{0}=100 \%$ (Fig. 8.26) and $\gamma_{0}=500 \%$ (Fig. 8.27). Appended to each figure is a representation of the form 


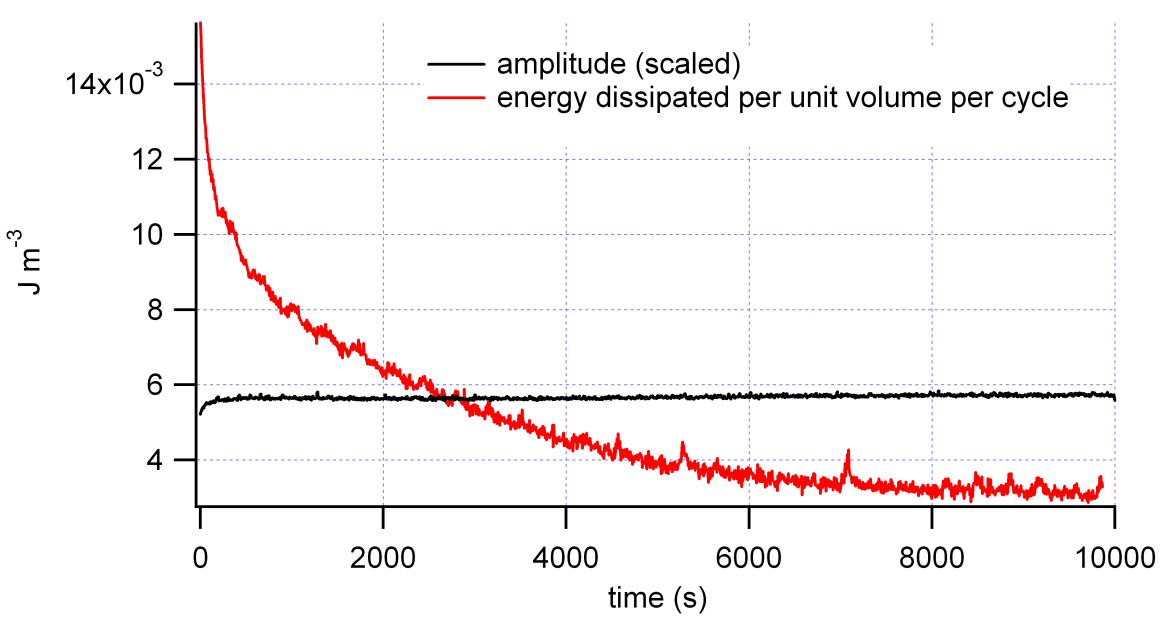

Figure 8.23: The evolution of the total energy dissipated per unit volume per cycle plotted against the rescaled stress amplitude for a strain amplitude $\gamma_{0}=1 \%$.

of the evolution of the amplitude of the stress response, scaled to fit each figure. It is interesting to note that for strain conditions defined by Fig. 8.15 as being in the linear region, the forms of the amplitude and energy dissipation evolutions are markedly different. This is understood to confirm a predominantly solid-like response from the material under such strain conditions.

When the applied strain amplitude is $\gamma_{0}=10 \%$, the energy dissipation initially drops rapidly, indicating a response that tends towards that of an elastic solid. The energy dissipated then quickly rises again suggesting a reverse of the process that is responsible for the short time response; the material response is more liquid-like. This rapid change can be interpreted as being due to an ensemble average of cage-breaking events: initially individual stars are sheared into each other, creating highly strained cages that eventually yield. The data of Fig. 8.15 suggests that $\gamma_{0}=10 \%$ is in the shearmelting regime but suggest no time frame over which the melting occurs. One possible interpretation of the data of Fig. 8.24 is that a time of $\sim 150 s$ is required to break the majority of cages before the onset of macroscopic flow. The comparatively long time for a microscopic process can be understood by noting that cage-breaking is a strainrelated phenomenon and the applied frequency of $1 \mathrm{rad} \mathrm{s}^{-1}$ means that in $\sim 150 \mathrm{~s}$, only $\sim 24$ strain cycles have been completed. With an upper strain limit of $\gamma_{0}=10 \%$, the time spent in conditions where the strain is large enough to break a cage is significantly shorter than $150 \mathrm{~s}$. The manner in which the form of the amplitude evolution matches 


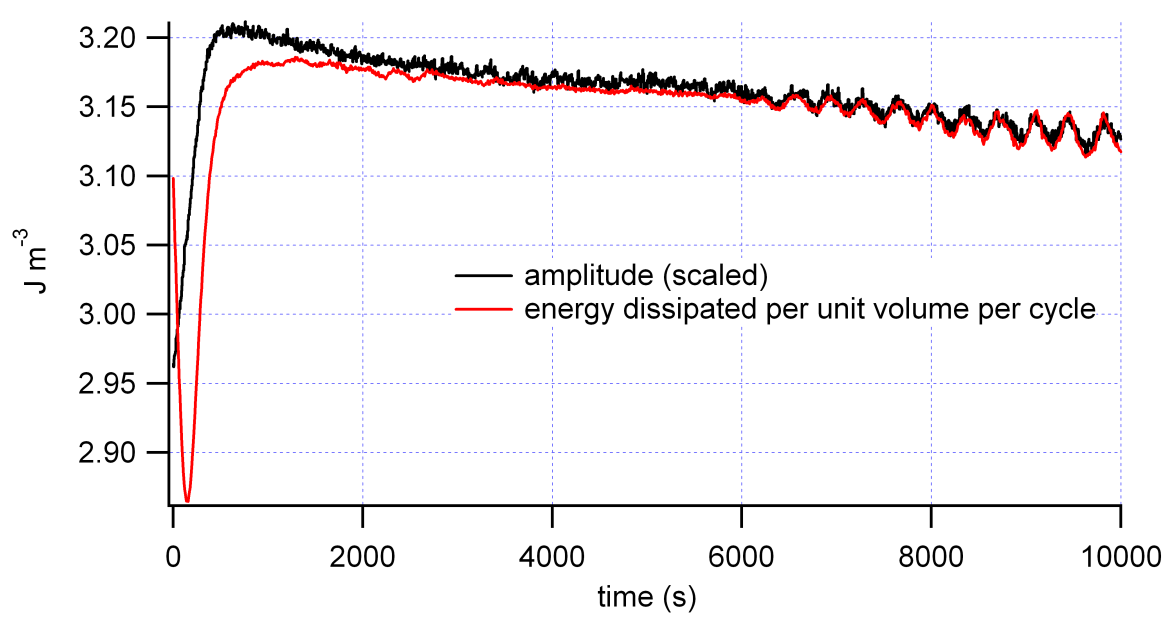

Figure 8.24: The evolution of the total energy dissipated per unit volume per cycle plotted against the rescaled stress amplitude for a strain amplitude $\gamma_{0}=10 \%$.

that of the evolution of the energy dissipation at longer times is strongly suggestive of macroscopic flow. It is interesting to note also that once the majority of cages have been broken, the general trend is for the amount of energy dissipated per unit volume per cycle to decrease. This is indicative of a slow stiffening of the material.

The data of Fig. 8.24 can be understood in terms of the response of a material whose constituents are undergoing cage straining followed quickly by cage breaking. The data of Fig. 8.15 suggest that $\gamma_{0}=20 \%$ is large enough to be in the shear-thinning regime where the cages are broken and macroscopic flow is predominant. The data of Fig. 8.25, and in particular the lack of correlation between the forms of the evolutions of the stress amplitude and the energy dissipated, suggests more complex physics than simply cage breaking and flow is responsible for the response when $\gamma_{0}=20 \%$. Like the response to $\gamma_{0}=10 \%$, the amount of energy in the $\gamma_{0}=20 \%$ response initially decreases rapidly. Unlike the response to $\gamma_{0}=10 \%$, the response to $\gamma_{0}=20 \%$ shows no corresponding rapid increase but instead slowly increases in a step-wise manner that is modulated by the $\sim 360 s$ oscillations attributed to the air conditioning temperature control cycle.

The high degree of correlation between the forms of the evolutions of the stress amplitude and energy dissipation for $\gamma_{0}=100 \%$ displayed in Fig. 8.26 and $\gamma_{0}=500 \%$ displayed in Fig. 8.27 is indicative of macroscopically flowing material. For the first $2000 s$ while being sheared with an amplitude of $\gamma_{0}=100 \%$, the dissipation of energy 


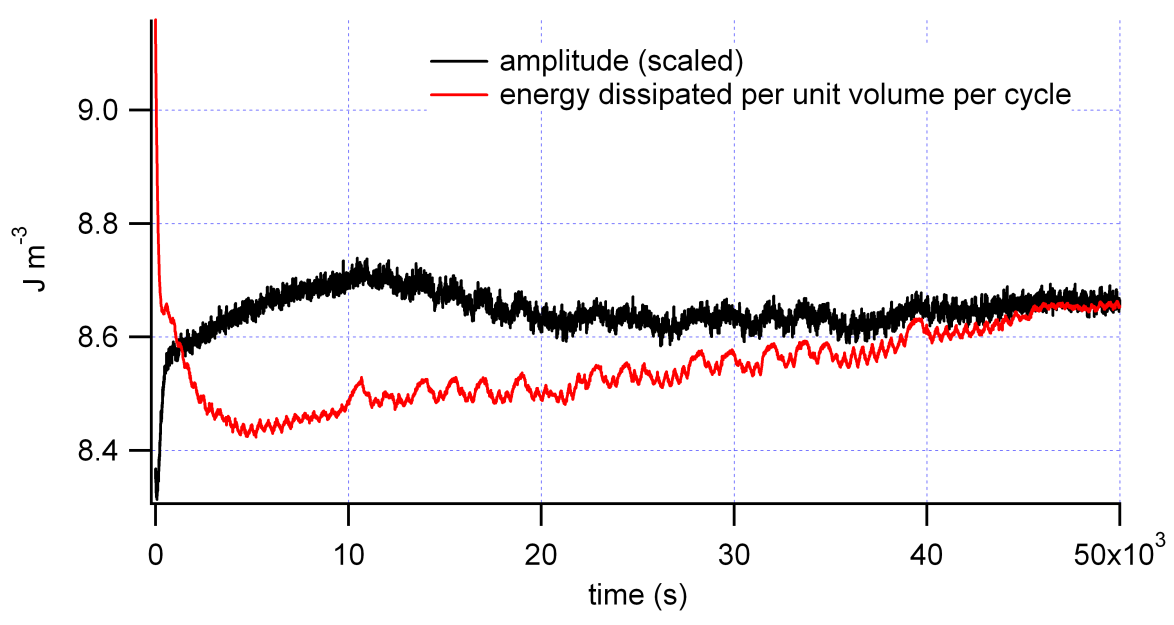

Figure 8.25: The evolution of the total energy dissipated per unit volume per cycle plotted against the rescaled stress amplitude for a strain amplitude $\gamma_{0}=20 \%$.

per unit volume per cycle increases; the response gets more liquid-like. After the first $2000 s$ at $\gamma_{0}=100 \%$ and for the entire experimental time at $\gamma_{0}=500 \%$, the energy dissipated per unit volume per cycle decreases; the material gets progressively stiffer.

\subsection{Discussion}

While much of the steady-state rheology has already been elucidated [29, 127, 126], the data and methods presented in this chapter correspond to the first attempt to understand the LAOS behaviour. One particular aspect of the steady-state start-up behaviour that is important in understanding these data is the stress overshoot observed for shear rates $\dot{\gamma}>0.001 \mathrm{~s}^{-1}$ with $t_{w} \sim 0$ shown in Fig. 8.28 (a). The characteristic shapes seen in the LAOS data could be interpreted as being an overshoot followed by a further increase in stress related to the increase in strain. Previous measurements have suggested a yield strain of $\sim 10 \%$ which would mean an overshoot would be expected for an oscillatory test with $\gamma_{0}=20 \%$. For strain amplitudes $\gamma_{0}>\sim 20 \%$ at an angular frequency of $1 \mathrm{rads}^{-1}$, the stress maximum occurs at negative strains which suggests that the absolute strain is not as important as the direction of strain once cages have been strain-broken.

The stress overshoot has been suggested as correlating to cage breaking and the 


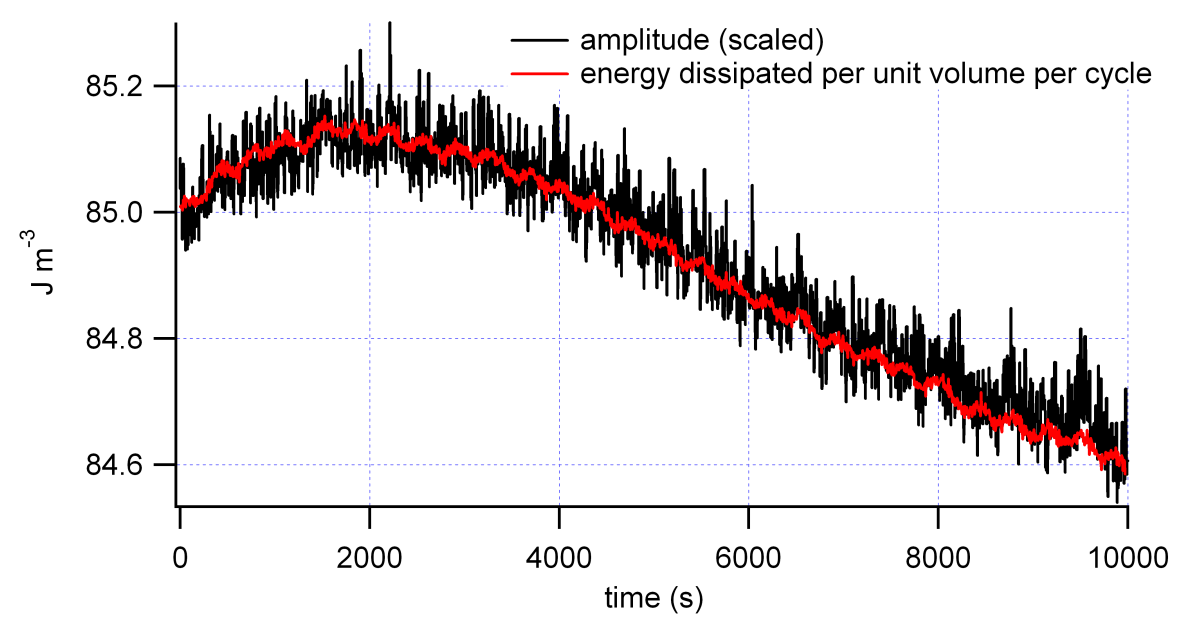

Figure 8.26: The evolution of the total energy dissipated per unit volume per cycle plotted against the rescaled stress amplitude for a strain amplitude $\gamma_{0}=100 \%$.

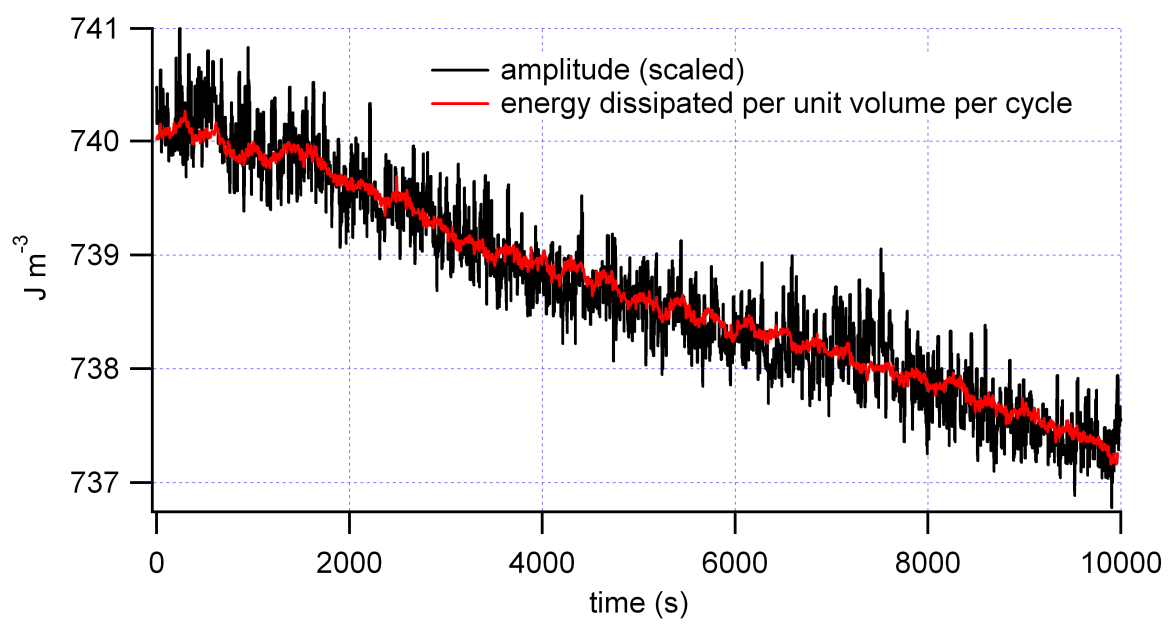

Figure 8.27: The evolution of the total energy dissipated per unit volume per cycle plotted against the rescaled stress amplitude for a strain amplitude $\gamma_{0}=500 \%$. 


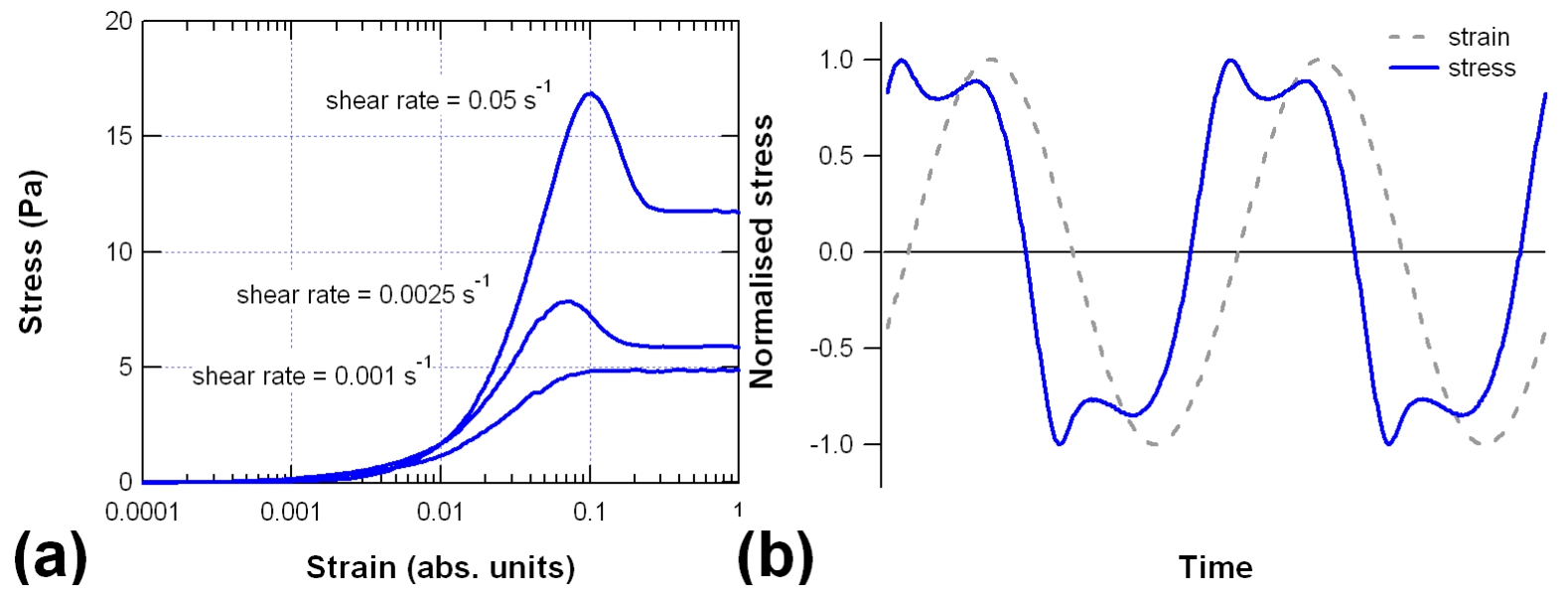

Figure 8.28: (a) The stress overshoot observed in steady-shear start-up experiments occurs for shear rates $\dot{\gamma}>0.001 \mathrm{~s}^{-1}$. (b) The stress response to an oscillatory strain of $\omega=1 \mathrm{rad} \mathrm{s}{ }^{-1}, \gamma_{0}=20 \%$. Note the first stress maximum occurs while the strain is still negative, but moving in the positive direction and the second stress maximum occurs at very nearly the maximum strain.

onset of flow [47] and the Cross model of eqn. 8.1 is based on the idea of formation and rupture of structural linkages. The quality of the fits achieved when the Cross model is applied to the oscillatory data shown above makes it reasonable to view the form of the stress response seen as part of the underlying flow curve that is traversed. This method gives a possible pathway of mapping the early-time evolution of the flow curve.

A two-peak shape exhibited by the stress response to LAOS has been previously reported by Chopra et al. in their study of the miscible polymer blend poly(styrene-comaleic anhydride)/poly(methyl methacrylate) and was attributed to the phase separation of the blend. A similar shaped response is reported here and fit with a Cross model in an extended Cox-Merz rule. It is suggested that such analysis allows the monitoring of the early-time evolution of the flow curve toward the steady-state form. Under this assumption, a two-peak LAOS response corresponds to a flow curve with a branch of negative slope - a branch that is unstable under steady-flow conditions. It has long been suggested that such branches are characteristic of spatial heterogeneities such as phase separations and shear bands, a phenomenon that has previously been reported for this system under steady-shear conditions at times much longer than a period of the LAOS investigated here. The evolution of symmetry factors reported here in the 
response to LAOS also suggests that this spatial heterogeneity changes as a function of time. At large strain amplitudes, where the two-peak shape is observed in the stress response to LAOS, the symmetry factors reported here tend towards the symmetric limits in discrete jumps.

A speculative picture is suggested to explain these results using the idea of clustering. Clustering has been attributed to the reversible vitrification upon heating in systems of stars [30], a phenomenon that is common to soft materials that can increase their volume fraction at constant number concentration. The idea of clustering has also been used in the discussion of the physics of the polymer-mediated melting phenomenon where large chains (with respect to the star arm) create an osmotic force that shrinks individual stars and eventually yields star-star clustering at high concentrations. With regards to the stress response to LAOS, the two-peak shape is highly suggestive of some degree of spatial heterogeneity. The smooth way the two-peak shape evolves and the constancy of the shape suggests the spatial heterogeneity is not macroscopic shear banding, but rather some other sort of small-scale heterogeneity that appears homogeneous on the large scale. This idea is consistent with clustering and is a subtle point that deserves more discussion. Macroscopic shear banding has been observed in this sample starting from identical initial conditions under steady shearing. Such banding takes on the order of $10^{3} s$ to occur under such conditions, whereas the spatial heterogeneity present in the LAOS response manifests itself after times on the order of $10 \mathrm{~s}$. The magnitude and evolution of the symmetry factors can be interpreted as suggesting a polydispersity in clusters.

There are two types of polydispersity possible in such situations; polydispersity in cluster size due to numbers of stars and polydispersity of cluster shape. Each change in a cluster would have a distinctly different manifestation in the parameters presented here. Assuming a uniform shape, an increase in cluster size would naturally decrease the amount of energy dissipated per unit volume per cycle, as depicted in the long time response displayed in Figs. 8.24, 8.26 and 8.27. Imagine a cigar-shaped cluster (this argument works for any nonspherical cluster shape but the cigar is an easy shape to visualise). Such a cluster would be able to store more energy along its major axis when strained compared to along its minor axis. However, this theoretical cluster would be subject to larger torques when undergoing oscillatory shear due to its asphericity. This would lead to a higher energy cost in remaining cigar shaped (nonspherical). Spherical clusters would have lower torques acting upon them and thus be more stable. For a 
constant number of stars in a group of clusters, the amount of energy dissipated per unit volume per cycle would increase as the average cluster became more spherical. This would also mean there is a decrease in the asymmetry of the stress response as any preferred strain direction disappears.

The data presented in section IV suggest that once cages are broken at strain amplitudes $\gamma_{0} \geq 10 \%$ at an angular frequency of $1 \mathrm{rad} \mathrm{s}^{-1}$, there is a tendency for the average cluster size to increase, as indicated by the long time decrease in energy dissipation. The exception to this general rule is the response to $\gamma_{0}=20 \%$. It is thought that in this case, cages are easily and quickly broken, leaving the system in a state of small, oddlyshaped and -sized clusters. These clusters undergo changes in both size and shape that act to increase the amount of energy dissipated by the material. These changes occur discretely which gives the characteristic stepwise response displayed in Figs. 8.13, 8.19 and 8.25 .

That the symmetry factors tend towards absolute symmetry suggests the polydispersity of clusters decreases as a function of time; that is, cluster sizes and shapes tend towards a common average size (which itself increases as a function of time) and a common spherical shape. If cluster size is the most important feature, the discrete nature of the changes in the symmetry factors suggests that significant fractions of cluster sizes are involved in those changes through a series of catastrophic events. This could mean that for cluster sizes on the order of tens of stars, changes occur by pairs or triplets of stars leaving larger clusters, or for smaller clusters of approximately ten stars, the changes could be indicative of individual stars breaking away from the clusters. The lack of turbidity in the sample, even at the longest times probed experimentally suggests the clusters formed are at least smaller than optical wavelengths. This puts an upper limit of $\sim 300 \mathrm{~nm}$, approximately sixty stars, on the diameter of the clusters. The mechanism that restricts cluster size to below this upper limit is thought to be a combination of thermal agitation and the changing sense of the mechanical shear. If cluster shape contributes more to the stress response than size, then the discrete changes suggest a 'clicking' of clusters into more spherical shapes. Most likely, a combination of size and shape is important in determining the stress response and so both processes will take place simultaneously, eventually leading towards clusters of relatively constant size and shape.

The system evolves into a macroscopically flowing state characterised by nearly uniform cluster size and shape under oscillatory shear, and into a shear banded state, 
where there are little or no restrictions on cluster formation, when submitted to steady shear. Under steady-shear conditions, the shearing is always in the same sense, meaning the torques applied to any newly-forming clusters are steady. When the material is sheared in an oscillatory fashion, cluster formation takes place in an environment where local torques change magnitude and direction, subjecting the newly-forming clusters to unequal forces. These unequal forces act to create more uniform cluster sizes and shapes in LAOS. The mechanism controlling cluster shape is therefore weaker under steady-shear conditions than under oscillatory conditions.

An idea put forward by Jabbari-Farouji et al. [103] can be applied to the current situation. Starting with identical initial conditions, it is possible to evolve the system through the free-energy landscape to a macroscopically shear banded state via the application of steady shear below a critical shear rate, a homogeneous liquid for steady shear above the critical rate and a state dominated by nearly-uniform sized and shaped clusters via the application of large amplitude oscillatory shear. 


\section{Chapter 9}

\section{Conclusions}

The flow and ageing properties of a system of ultrasoft colloidal spheres have been studied. The flow properties have been studied using conventional rheological techniques, which give the bulk response of the material, in conjunction with non-invasive rheo-NMR velocimetry, which attaches spatial information to the measured response. Experimentally altered parameters included shear rate, $\dot{\gamma}$, shear stress, $\sigma$, waiting time, $t_{w}$ and temperature, $T$. The ultrasoft colloidal spheres investigated are star polymers with a nominal functionality, $f$, of $f=128$ arms suspended in squalene at two concentrations: 1.5 and $2 c^{*}$, where $c^{*}$ is the overlap concentration.

An oscillatory preshearing protocol has been established and implemented that has allowed the system to be placed in a reproducible fluidised initial condition. The protocol enabled a careful study of the changing mechanical response of the material as a function of time. Using the protocol, it has been shown that under controlled shear rate conditions, the system evolves from a shear-thinning fluid under all shear rates at times $t<\sim 1000 s$ to a material whose response is characterised by a plateau in the flow curve at a stress $\sigma_{c}=8 \pm 0.25 P a\left(c=1.5 c^{*}\right), 11 \pm 0.25 P a\left(c=2 c^{*}\right)$, for all shear rates measured below $\dot{\gamma}=0.05 \mathrm{~s}^{-1}\left(c=1.5 c^{*}\right), 0.035 \mathrm{~s}^{-1}\left(c=2 c^{*}\right)$ for times $t>\sim 3 \times 10^{4} \mathrm{~s}$. The evolution of the stress plateau alludes to, but cannot definitively confirm, the presence of spatially heterogenous flow. The time at which the stress response of the material reaches the critical value $\sigma_{c}$ has been shown to be an approximately logarithmic function of shear rate, with the material sheared at shear rates closer to the critical rate, $\dot{\gamma}$, requiring more time to reach $\sigma_{c}$.

The form of the stress evolution under the application of a constant shear-rate has been shown to follow one of three different behaviours: 
- for rates $\dot{\gamma}<\dot{\gamma}_{\beta}$, the stress rises to a pseudo-steady value $\sigma_{p-s}$ and remains at this value for a time on the order of $1000 \mathrm{~s}$ before rising to a common critical stress $\sigma_{c}$

- for rates $\dot{\gamma}_{\beta}<\dot{\gamma}<\dot{\gamma}_{c}$, the stress rises and overshoots to $\sigma_{\text {plas }}$ before relaxing back to $\sigma_{p-s}$. Further, $\sigma_{\text {plas }}-\sigma_{p-s}$ increases as $\dot{\gamma}-\dot{\gamma}_{\beta}$ increases. The stress remains at $\sigma_{p-s}$ for time on the order of $1000 s$ before rising to to the common critical stress $\sigma_{c}$

- for rates $\dot{\gamma}_{c}<\dot{\gamma}$, the stress overshoots to $\sigma_{\text {plas }}$ before relaxing back to $\sigma_{s-s}$. Further, $\sigma_{\text {plas }}-\sigma_{s-s}$ increases as $\dot{\gamma}-\dot{\gamma}_{c}$ increases (the subscript s-s is used in this case because there is no pseudo-steady state, only a steady state.).

The values of $\dot{\gamma}_{\beta}$ have been found to be $\dot{\gamma}_{\beta}=\sim 0.005 s^{-1}\left(c=1.5 c^{*}\right), \sim 0.001 s^{-1}(c=$ $\left.2 c^{*}\right)$.

It has also been shown, by bypassing the controlling software of the rheometer, that the preshearing protocol leaves the system in a stressed state, with the stress relaxing to $\sim 10 \%$ of its initial value after $t_{w}=\sim 200 \mathrm{~s}$ of spontaneous evolution (no shear). When sheared after a waiting time $t_{w}$, the maximum stress achieved at the plastic overshoot, $\sigma_{\text {plas }}$ rises as a power-law with waiting time, $t_{w}$, indicating that the material begins to rearrange to a stiffer configuration immediately following preshearing if left to evolve spontaneously under zero-shear conditions.

A series of constant-stress experiments have shown that when stressed at a stress $\sigma<\sigma_{c}$ after a time $t_{w}$ following fluidisation, the material initially acquires strain at a slower rate when $t_{w}$ is longer. For waiting times $t_{w} \geq \sim 5000 \mathrm{~s}$, the material acquires strain at a rate that is several orders of magnitude slower than that exhibited when $t_{w}=0$. When the application of stress is ceased, the material recovers an increasing proportion of its acquired strain when $t_{w}$ is longer. When the waiting time is on the order of $t_{w} \approx 10^{4} s, 97 \%$ of the acquired strain is recovered. This suggests once again that a rearrangement to a configuration, the response of which resembles a perfectly elastic solid, takes place following fluidisation when the material is allowed to evolve spontaneously. Furthermore, a stress of $\sigma \geq \sigma_{c}$ is required to destroy such a configuration.

When a uniform strain of $1 \%$ is applied to the material after a waiting time $t_{w}$, the evolution to the more rigid state manifests itself as an increase in the stress developed in the system and a series of discrete jumps relaxing that stress. An ideal glass would 
retain that stress indefinitely. The state formed can thus be thought of as a short-lived glass lasting approximately $500 \mathrm{~s}$.

Conventional rheological experiments have also shown that the transition to the stiffer state takes place under oscillatory shear, provided the critical stress is not exceeded, as indicated by a dramatic change in dynamic moduli. The lowering of the broad minimum exhibited by the loss modulus as a function of age is highly suggestive of an increase in size of the constituent particles, adding more weight to the argument for cluster formation.

Spatially resolved rheo-NMR velocimetry experiments clearly show the material evolves into a shear-banded structure when sheared at a constant gap-average shear rate such that the range of stresses across the Couette geometry gap includes stresses below the critical stress $\sigma_{c}$. The velocity of the material in the lower band was below the experimental uncertainty of $1.5 \mu \mathrm{m} \mathrm{s}^{-1}$ and so was assumed, for convenience, to be $0 s^{-1}$. The growth of the zero-shear band was found to follow a Gompertzian growth curve, suggesting the structure responsible for the zero-shear band grows at a rate proportional to its size. The proportionality decreases as the size of the structure approaches an upper limit. This has been interpreted as further supporting the concept of cluster growth and requires a limiting mechanism. The results of the conventional rheology experiments when examined simultaneously with the velocimetry results suggest that the size and growth rate of the structure responsible for the zeroshear band is limited by the stress inherent in the system. Indeed, the relative proportion of gap displacement has been found to be dependent on the applied gap-average shear rate such that higher rates, which correspond to higher stresses on the material, lead to smaller proportions of the sample in the zero-shear band.

${ }^{2} \mathrm{H}$ NMR spectroscopy on per-deuterated stars has shown a complete lack of alignment in the arms of the stars immediately following fluidisation and also in the jammed state.

Physical insight has been gained by the application of a theoretical model. The model is based on a dual minimum stress-dependent free-energy landscape. One minimum is at high 'fluidity', corresponding to the fluid state, the other at zero fluidity, corresponding to the solidified state. Stresses higher than the critical stress make the high-fluidity state the global minimum and stresses below the critical stress make the zero fluidity state the preferred one. The mechanical fluidisation protocol initially places the sample in a state of high fluidity. Energy 'kicks' are given to the system by a 
stress-dependent noise term that is designed to mimic the combination of thermal and rearrangement energies present in such soft systems. The model replicates the main features of the experimental results; it predicts an evolution from a shear-thinning flow curve to one dominated by a stress plateau and also replicates the form of the velocity profiles seen in rheo-NMR experiments. The model also offers an explanation as to why the stress plateau takes longer to reach at rates/stresses closer to, but still less than, the critical values: the energy barrier that separates the two free-energy minima is largest when the stress is closer to the critical stress, while necessarily keeping the zero-fluidity state the global minimum.

Investigations into the response to LAOS have shown a characteristic decrease in the stress of the material that can be fit with a modified Cross model. These results are interpreted as supporting the idea of cage-breaking in the onset of flow and also provide a way of watching the early-time evolution of the flow curve. The decrease of the stress is also interpreted as providing further evidence of some form of spatial heterogeneity in the flow of the material. Further, the response to LAOS has been shown not to be a frieze group, as implicitly assumed in the literature. The degree to which the response does constitute a frieze group, i.e. the symmetry of the response to LAOS, has been followed by way of two parameters, the energy-dissipation ratio and the path-length difference. Both of these parameters give a scalar measure of symmetry between the positive and negative-stress lobes. When followed in tandem they provide a symmetry vector that has proved useful in determining the ideality of the response. The evolution of these parameters has been interpreted as providing further support for cluster formation. Further, the tendency of the symmetry parameters towards the symmetric limit is thought to be caused by the reduction in polydispersity of cluster sizes and shapes. The symmetry parameters have also been interpreted as giving a more accurate gauge of the limits of the linear regime as compared with the response of the dynamic moduli.

The part of the soft matter universe populated by densely-packed star polymers may be queerer than we had supposed, as evidenced by the previously unseen behaviours presented in this thesis, but it is certainly not, as Haldane had suspected, queerer than we can suppose. 


\subsection{Concluding remarks}

The conventional rheological component of this thesis (the work presented in chapters 5 and 8) was carried out at the Foundation Of Research and Technology, Hellas (FORTH), in Greece, under the supervision of Prof. Dimitris Vlassopoulos as part of an ongoing collaboration. The work was carried out in two separate visits with the work of chapter 5 taking place in one six-week period in 2005 and the work of chapter 8 being completed in one two-week visit in 2007. At the end of the first visit, a series of experiments were suggested as being interesting based on the results presented here. The experiments suggested included detailed creep measurements above and below $\sigma_{c}$ for the same system and further stress relaxation measurements. The suggestions of the author formed the basis of a project for a graduate student of Dr. George Petekidis, Christina Christopoulou. The results of these experiments suggest a lower branch of the flow curve exists around shear rates of $\dot{\gamma} \sim 10^{-6} s^{-1}$.

The major difference between the stars studied in this work and the linear chains and hard spheres that dominate the literature is the soft interaction potential of the stars. It is the soft potential, the ability of the arms of stars to interpenetrate, that ultimately leads to the extraordinary behaviours reported in this thesis. To study materials that possess potentials of varying degrees of softness is the obvious next step following this work. It would be particularly interesting to study the long-time behaviours and critical stresses/strain rates to determine just how 'softness' of the interaction potential plays a part in the dynamics of soft materials. To study such materials with techniques that allow temporal resolutions of milliseconds and spatial resolutions of sub-microns would enable some of the speculation ventured here to be confirmed or dispelled. Rheo-NMR is capable of such spatial and temporal resolution, but limited sample quantities (sample sizes used in this thesis were on the order of milligrams) restricted the scope of this thesis. Stars are notoriously difficult and time-consuming to produce, so having a steady supply of easily produced soft-interaction materials would facilitate testing larger sample sizes.

It would be interesting if a soft material could be produced that had a soft interaction potential similar to the stars studied here, but also possessed a simple morphology such that experiments could determine physical alignments.

While the model presented in chapter 7 replicates many of the features of the ex- 
perimental responses, it does so in a limited way. Many of the parameters are chosen so that the results of the calculations match the data. The experimental results indicate that many of the model parameters ought to be functions of one, or several, experimentally controlled variables. Further work on the model presented would be necessary to reveal these relations and may yield a deeper understanding than that gathered here.

The interesting results displayed in chapter 8 form the first ever detailed investigation into the nature of the symmetry of the response of a material to LAOS. As such, it would be extremely interesting to investigate more soft materials using a similar analysis to find just how reasonable the apparently global, but in the current case, false, assumption of LAOS responses being $\infty x$ (step) frieze groups is. Such experiments ought to encompass not just temporal changes, but also strain amplitude and frequency. 


\section{Appendix A: Frieze Groups}

A frieze group is a mathematical concept that classifies geometrical patterns on twodimensional surfaces which are repetitive in one direction, based on symmetries present in the pattern. Formally, a frieze group is a class of infinite discrete symmetry groups for patterns on an infinitely wide rectangle, hence a class of groups of isometries of a plane, or of a strip. Frieze groups are related to the more complex wallpaper groups, but whereas wallpaper groups extend over the entire plane, frieze groups extend over a strip only. Because of the spatial limitations on frieze groups, the isometries allowed for frieze groups only include translations in one direction, reflections parallel and perpendicular to the direction of propagation of the frieze and rotations or order two. A rotation is said to be of order $n$ if the smallest angle of symmetry about a point is $2 \pi / n$. A rotation of order two is thus a rotation of $\pi$ radians, or $180^{\circ}$. There exist seven different frieze groups that are composed of combinations of these isometries.

The system used to identify the frieze groups used in this thesis was popularised by the mathematician John Horton Conway and is called 'orbifold notation'. An orbifold (a conjunction of "orbit manifold") is a mathematically generalised manifold. In orbifold notation, each frieze group is denoted by a finite string made of positive integers, the infinity symbol, $\infty$, the asterisk, $*$, and the symbol $x$, called a miracle. Each symbol has a meaning attached to it: an integer to the left of an asterisk indicates a rotation of order $n$ around a point, an integer to the right of an asterisk indicates a transformation of order $2 n$ which rotates around a point and reflects through a line, an $x$ indicates a glide reflection (a combination of a reflection and translation) and the $\infty$ symbol indicates infinite rotational symmetry about a line. Conway also attached names to each frieze, based on the pattern made by footprints as illustrated in Fig. 9.1. 
Using orbifold notation, the seven frieze groups are:

$\infty \infty$ (hop) Translations only.

$\infty x$ (step) Glide reflections and translations.

$\infty *$ (jump) Translations and a reflection in the horizontal axis.

$* \infty \infty$ (sidle) Translations and reflections across vertical lines.

$22 \infty$ (spinning hop) Translations and $180^{\circ}$ rotations.

$2 * \infty$ (spinning sidle) Reflections across certain vertical lines, glide reflections, translations and rotations.

$* 22 \infty$ (spinning jump) Translations, glide reflections, reflections in both axes and $180^{\circ}$ rotations.

Having established what constitutes a frieze group we are now in a position to make some comments about the assumptions made in the literature (as of January 2009) regarding material responses to LAOS. It is universally assumed the response of materials to large-amplitude oscillatory shear constitutes a $\infty x$ (step) frieze group (a linear response to small-amplitude oscillatory shear is assumed to constitute a $2 * \infty$ (spinning sidle) frieze group, just as a graph of $\sin (t)$ as a function of $t$ does). This is explicitly stated by Cho et al. [123] where they make the substitutions $x=\gamma$ and $y=\dot{\gamma} / \omega$ and state the assumption in the form $\sigma(-x,-y)=-\sigma(x, y)$. It appears as though this assumption is 'fundamental' enough for others not to feel the need to state. This assumption is shown incorrect in the present case, in chapter 8.

The method of Klein et al. when applied to the experimental data of Fig. 8.2 produces a $22 \infty$ (spinning hop) frieze group as seen in Fig. 8.3 and thus does not produce a good description of the material's response. 

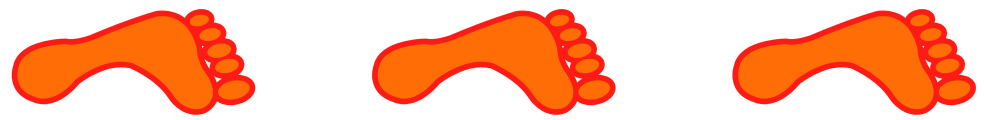

(a)
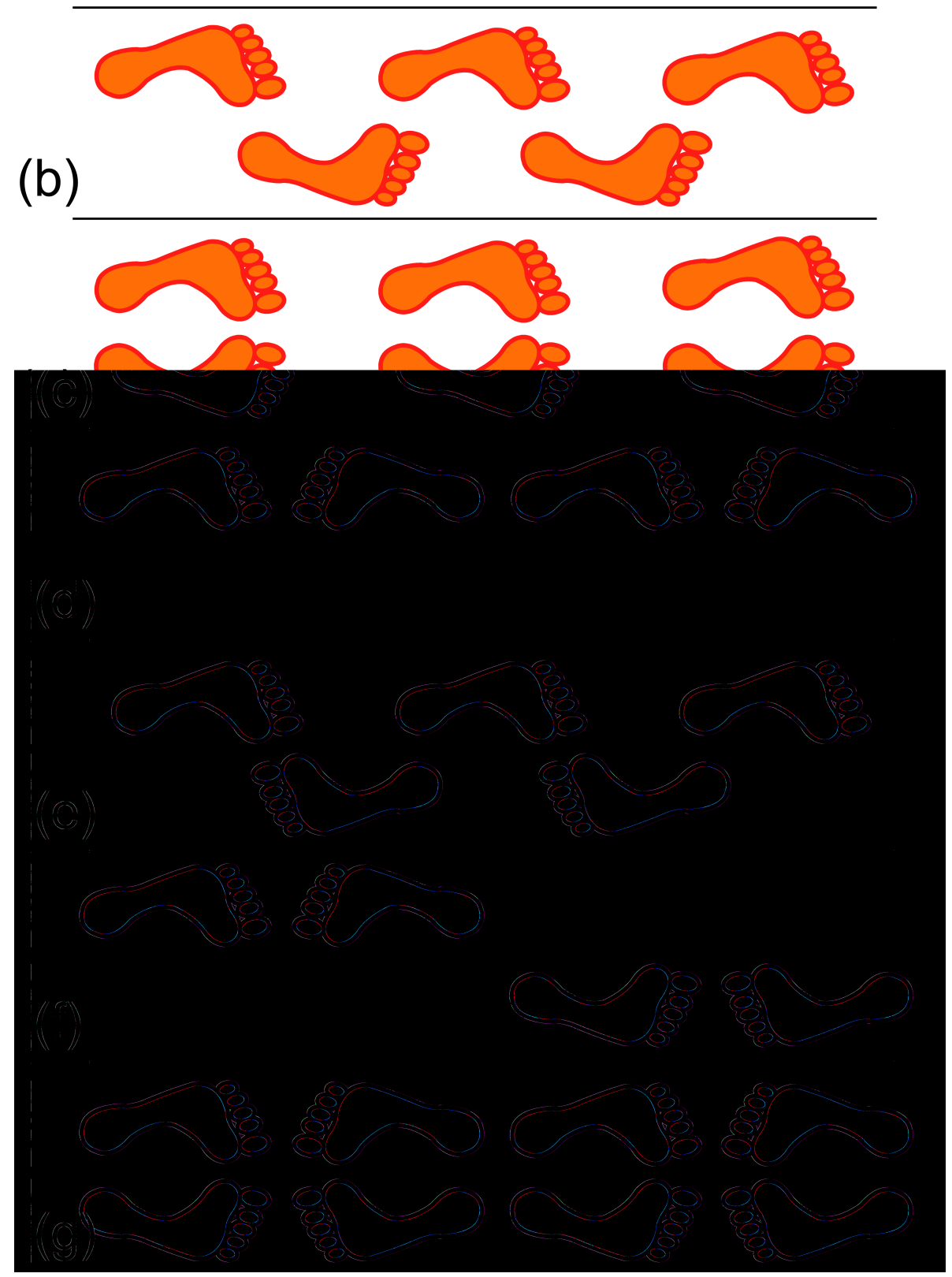

Figure 9.1: The seven frieze groups illustrated with a single left foot motif. Using orbifold notation, these are denoted (a) $\infty \infty$ (hop), (b) $\infty x$ (step), (c) $\infty *$ (jump), (d) $* \infty \infty$ (sidle), (e) $22 \infty$ (spinning hop), (f) $2 * \infty$ (spinning sidle) and (g) $* 22 \infty$ (spinning jump). It is assumed universally, outside of this work, that the stress response of all materials to oscillatory shearing constitutes an $\infty x$ (step) frieze group. 



\section{References}

[1] J. B. S. Haldane, Possible Worlds and Other Papers, Ayer Co Pub, reprint edition edition, 1977.

[2] J. F. H. H. A. Barnes and K. Walters, An Introduction to Rheology, Elsevier, Amsterdam, 1st edition, 1989.

[3] T. E. Faber, Fluid Dynamics for Physicists, Cambridge University Press, Cambridge, UK, 1st edition, 1995.

[4] R. G. Larson, The Structure and Rheology of Complex Fluids, Oxford University Press, New York, 1st edition, 1999.

[5] Taken from an after dinner talk presented by Marcus Reiner at the Fourth International Congress on Rheology, Brown University, Providence, Rhode Island, August 26-30, 1963.

[6] M. M. Cross, J. Colloid Sci. 20, 417 (1965).

[7] C. L. M. H. Navier, Mem. Acad. Sci. Inst. Fr. 6, 839 (1827).

[8] P. G. de Gennes, C. R. Acad. Sci. 288B, 219 (1979).

[9] R. Pit, H. Hervet, and L. Leger, Phys. Rev. Lett. 85, 980 (2000).

[10] V. S. J. Craig, C. Neto, and D. R. M. Williams, Phys. Rev. Lett. 87, 054504 (2001).

[11] A. Abragam, Principles of Nuclear Magnetism, Clarendon Press, Oxford, 1961.

[12] C. Slichter, Principles of Magnetic Resonance, Springer, Berlin, 3rd edition, 1990.

[13] E. Fukushima and S. Roeder, Experimental pulse NMR A nuts and Bolts Approach, Addison-Wesley Publishing Company, Inc., 1981. 
[14] P. T. Callaghan, Principles of Nuclear Magnetic Resonance Microscopy, Oxford Science Publishing, Oxford, 1st edition, 1991.

[15] B. Blumich, NMR Imaging of Materials, Oxford University Press, Oxford, 2000.

[16] P. Morris, Nuclear Magnetic Resonance Imaging in Medicine and Biology, Clarendon press, Oxford, 1986.

[17] R. Kimmich, NMR-Tomography, Diffusometry, Relaxometry, Springer-Verlag, Berlin Heidelberg, Germany, 1997.

[18] M. G. Mayer and J. H. D. Jensen, Elementary theory of nuclear structure, John Wiley, New York, 1955.

[19] E. L. Hahn, Physical Review 80, 580 (1950).

[20] H. Y. Carr and E. M. Purcell, Physical Review 94, 630 (1954).

[21] S. Meiboom and D. Gill, Rev. Sci. Instr. 29, 630 (1959).

[22] J. Karger and W. Heink, J. Mag. Res. 51, 1 (1983).

[23] M. E. Cates, J. P. Wittner, J.-P. Bouchaud, and P. Claudin, Phys. Rev. Lett. 81, 1841 (1998).

[24] G. S. Grest, J. L. Fetters, J. S. Huang, and D. Richter, Adv. Chem. Phys. XCIV, 67 (1996).

[25] J. Roovers, L.-L. Zhou, P. M. Toporowski, M. van der Zwan, H. Iatrou, and N. Hadjichristidis, Macromolecules 26, 4324 (1993).

[26] C. N. Likos, H. Lowen, M. Watzlawek, B. Abbas, O. Jucknischke, J. Allgaier, and D. Richter, Phys. Rev. Lett. 80, 4450 (1998).

[27] D. Vlassopoulos, G. Fytas, T. Pakula, and J. Roovers, J. Phys. Condens. Matter 13, R855 (2001).

[28] D. Vlassopoulos, J. POLYM. SCI. PART B: POLYM. PHYS 42, 2931 (2004).

[29] M. E. Helgeson, N. J. Wagner, and D. Vlassopoulos, J. Rheol. 51, 297 (2007). 
[30] M. Kapnistos, D. Vlassopoulos, G. Fytas, K. Mortensen, G. Fleischer, and J. Roovers, Phys. Rev. Lett. 85, 4072 (2000).

[31] W. M. Holmes, P. T. Callaghan, D. Vlassopoulos, and J. Roovers, J. Rheol. 48, 1085 (2004).

[32] M. Doi and S. F. Edwards, The Theory of Polymer Dynamics, Oxford University Press, New York, 1986.

[33] S. F. Edwards, Proc. Phys. Soc. 92, 9 (1967).

[34] S. F. Edwards, Brit. Polym. J 9, 140 (1977).

[35] P. G. de Gennes, J. Chem. Phys. 55, 572 (1971).

[36] P. N. Pusey and W. van Megen, Nature 320, 340 (1986).

[37] J. P. Hansen, D. Levesque, and Z.-J. J., editors, Liquids, Freezing and Glass Transition, chapter Colloidal Suspensions, North Holland, Amsterdam, 1991.

[38] E. G. Cohen and I. M. de Schepper, J. Stat. Phys. 63, 241 (1991).

[39] J. Frenkel, Kinetic Theory of Liquids, The Clarendon Press, Oxford, 1946.

[40] D. S. Pearson and E. Helfand, Macromolecules 17, 888 (1984).

[41] R. C. Ball and T. C. B. McLeish, Macromolecules 22, 1911 (1989).

[42] S. T. Milner and T. C. B. McLeish, Macromolecules 30, 2159 (1997).

[43] R. H. Colby and M. Rubenstein, Macromolecules 23, 2753 (1990).

[44] L. Ramos and L. Cipelletti, Phys. Rev. Lett. 87 (2001).

[45] J. Ren, B. F. Casanueva, C. A. Mitchell, and R. Krishnamoorti, Macromolecules 36, 4188 (2003).

[46] L. Cipelletti, S. Manley, R. Ball, and D. Weitz, Phys. Rev. Lett. 84 (2000).

[47] C. Derec, G. Ducouret, A. Ajdari, and F. Lequeux, Phys. Rev. E 67 (2003).

[48] V. Viasnoff and F. Lequeux, Rejuvenation and overaging in a colloidal glass under shear, 2002. 
[49] V. Viasnoff, S. Jurine, and F. Lequeux, Faraday Discuss. 123, 253 (2003).

[50] E. H. Purnomo, D. van den Ende, J. Mellema, and F. Mugele, Europhys. Lett. 76, 74 (2006).

[51] A. Knaebel, M. Bellour, J. Munch, V. Viasnoff, F. Lequeux, and J. Harden, Europhys. Lett. 52 (2000).

[52] M. Bellour, V. Viasnoff, L. Collins, A. Knaebel, J. Munch, F. Lequeux, and J. Harden, Abs. Pap. Amer. Chem. Soc. 219 (2000).

[53] R. Bandyopadhyay, D. Liang, H. Yardimci, D. Sessoms, M. Borthwick, S. Mochrie, J. Harden, and R. Leheny, Phys. Rev. Lett. 93, 228302 (2004).

[54] D. Bonn, S. Tanase, B. Abou, H. Tanaka, and J. Meunier, Phys. Rev. Lett. 89 (2002).

[55] R. D. Leonardo, S. Gentilini, F. Ianni, and G. Ruocco, Journal of Non-Crystalline Solids 352, 49284933 (2006).

[56] F. Schosseler, S. Kaloun, M. Skouri, and J. P. Munch, Phys. Rev. E 73 (2006).

[57] C. Wilhelm, F. Elias, J. Browaeys, A. Ponton, and J.-C. Bacri, Phys. Rev. E 66 (2002).

[58] M. Cloitre, R. Borrega, and L. Leibler, Phys. Rev. Lett. 85 (2000).

[59] L. Cipelletti, L. Ramos, S. Manley, E. Pitard, D. Weitz, E. Pashkovski, and M. Johansson, Faraday Discuss. 123, 237 (2003).

[60] R. Hohler, S. Cohen-Addad, and A. Asnacios, Europhys. Lett. 48, 93 (1999).

[61] P. Bursac, G. Lenormand, B. Fabry, M. Oliver, D. Weitz, V. Viasnoff, J. Butler, and J. Fredberg, Nature Materials 4, 557 (2005).

[62] L. Cipelletti and L. Ramos, J. Phys.: Condens. Matter 17, R253R285 (2005).

[63] C. Derec, A. Ajdari, and F. Lequeux, Eur. Phys. J. E 4, 355361 (2001).

[64] S. Fielding, P. Sollich, and M. E. Cates, J. Rheol. 44, 323 (2000).

[65] L. Varnik, L. Bocquet, and J.-L. Barrat, J. Chem. Phys. 120 (2004). 
[66] M. M. Britton and P. T. Callaghan, Phys. Rev. Lett. 78, 4930 (1997).

[67] M. R. Lopez-Gonzalez, W. Holmes, P. T. Callaghan, and P. J. Photinos, Phys. Rev. Lett. 93, 268302 (2004).

[68] P. Coussot, J. S. Raynaud, F. Bertrand, P. Moucheront, J. P. Guilbaud, H. T. Huynh, S. Jarny, and D. Lesueur, Phys. Rev. Lett. 88, 218301 (2002).

[69] P. Tapadia and S.-Q. Wang, Phys. Rev. Lett. 91, 198301 (2003).

[70] C. Pujolle-Robic and L. Noirez, Nature 409, 167 (2001).

[71] L. Isa, R. Besseling, and W. C. K. Poon, Phys. Rev. Lett. 98, 198305 (2007).

[72] N. Huang, G. Ovarlez, F. Bertrand, S. Rodts, P. Coussot, and D. Bonn, Phys. Rev. Lett. 94, 028301 (2005).

[73] P. C. F. Moller, J. Mewis, and D. Bonn, Soft Matter 2, 274 (2006).

[74] B. A. Isner and D. J. Lacks, Phys. Rev. Lett. 96, 025506 (2006).

[75] G. McKenna, J. Phys.: Condens. Matter 15, S737 (2003).

[76] K. Miyazaki, H. M. Wyss, D. A. Weitz, and D. R. Reichman, Europhys. Lett. 75, 915921 (2006).

[77] V. Carrier and G. Petekidis, Non-linear rheology of colloidal glasses of soft coreshell particles, 2007.

[78] T. G. Mason and D. A. Weitz, Phys. Rev. Lett. 75, 2770 (1995).

[79] G. Petekidis, D. Vlassopoulos, and P. N. Pusey, J. Phys.: Condens. Matter 16, S3955S3963 (2004).

[80] G. Petekidis, D. Vlassopoulos, and P. N. Pusey, Faraday Discuss. 123, 287 (2003).

[81] O. Diat, D. Roux, and F. Nallet, J. Phys. (France) 3, 1427 (1993).

[82] E. Eiser, F. Molino, G. Porte, and O. Diat, Phys. Rev. E 61, 2020 (2002).

[83] D. Bonn, P. Coussot, H. T. Huynh, B. F., and G. Debregeas, Europhys. Lett. 59, $786792(2002)$. 
[84] C. Monthus and J.-P. Bouchaud, J. Phys. A: Math. Gen. 29, 3847 (1996).

[85] J. P. Bouchaud, J. Phys. I(France) 2, 1705 (1992).

[86] P. Sollich, F. Lequeux, P. Hebraud, and M. E. Cates, Phys. Rev. Lett. 78, 2020 (1997).

[87] P. Sollich, Phys. Rev. E 58, 738 (1998).

[88] E. Stiakakis, D. Vlassopoulos, B. Loppinet, J. Roovers, and G. Meier, Phys. Rev. E 66, 051804 (2002).

[89] K. N. Pham, G. Petekidis, D. Vlassopoulos, S. U. Egelhaaf, W. U. K. Poon, and P. N. Pusey, Yielding behavior of repulsion- and attraction-dominated colloidal glasses, 2007.

[90] P. T. Callaghan, Rep. Prog. Phys. 62, 599 (1999).

[91] B. Gompertz, Philos. Trans. R. Soc. 115, 513 (1825).

[92] J. W. Curtsinger, H. H. Fukui, D. R. Townsend, and J. W. Vaupel, Science 258, 461 (1992).

[93] R. L. Buchanan, R. C. Whiting, and W. C. Damert, Food Microbiology 14, 313 (1997).

[94] E. Otterlei, G. Nyhammer, A. Folkvord, and S. O. Stefansson, Canadian Journal of Fisheries and Aquatic Sciences 56, 2099 (1999).

[95] P. Stoll, J. Weiner, and B. Schmid, Ecology 75, 660 (1994).

[96] M. Marusic, Z. Bajzer, and J. Freyer, Cell Proliferation 27, 73 (1994).

[97] T. D. Reichling and R. Z. German, Journal of Nutrition 130, 2326 (2000).

[98] R. H. Luecke, W. D. Wosilait, and J. F. Young, International Journal of BioMedical Computing 39, 337 (1995).

[99] A. Cardenas, M. Garcia-Molina, S. Sales, and J. Capmany, Journal of Lightwave Technology 22, 2460 (2004). 
[100] G. Jarne, J. Sanchez-Choliz, and F. Fatas-Villafranca, The Electronic Journal of Evolutionary Modeling and Economic Dynamics (2005).

[101] L. Egghe and I. K. Ravichandra Rao, Scientometrics 25, 5 (1992).

[102] A. Lutti and P. T. Callaghan, J. Mag. Res. 187, 251 (2007).

[103] S. Jabbari-Farouji, G. H. Wegdam, and D. Bonn, Phys. Rev. Lett. 99, 065701 (2007).

[104] H. Bassler, Phys. Rev. Lett. 58, 767 (1987).

[105] R. Richter and H. Bassler, J. Cond. Mat. Phys. 2, 2273 (1990).

[106] J. C. Dyre, Phys. Rev. Lett. 58, 792 (1987).

[107] T. Vilgis, J. Cond. Mat. Phys. 2, 3667 (1990).

[108] T. Odagaki, J. Matsui, and Y. Hiwatari, Physica 204A, 464 (1994).

[109] T. Odagaki, Phys. Rev. Lett. 75, 3701 (1995).

[110] J. P. Bouchaud, A. Comtet, and C. Monthus, J. Physique I 5, 1521 (1995).

[111] E. Dickinson, An Introduction to Food Colloids, Oxford University Press, Oxford, 1992.

[112] S. D. Holdsworth, Trans. Inst. Chem. Eng. 71, 139 (1993).

[113] M. R. Mackley, T. J. Marshall, J. B. A. Smeulders, and F. D. Zhao, Chem. Eng. Sci. 49, 25512565 (1994).

[114] R. J. Ketz, R. K. Prudhomme, and W. W. Graessley, Rheol. Acta 27, 531 (1988).

[115] S. A. Khan, C. A. Schnepper, and R. C. Armstrong, J. Rheol. 32, 69 (1988).

[116] P. Panizza, D. Roux, V. Vuillaume, C. Y. D. Lu, and M. E. Cates, Langmuir 12, 248 (1996).

[117] H. Hoffmann and A. Rauscher, Colloid Polym. Sci. 271, 390 (1993).

[118] T. G. Mason, J. Bibette, and D. A. Weitz, Phys. Rev. Lett. 75, 2051 (1995).

[119] G. Picard, G. Ajdari, L. Bocquet, and F. Lequeux, Phys. Rev. E 66, 051501 (2002). 
[120] P. Coussot, Q. D. Nguyen, H. T. Huynh, and D. Bonn, Phys. Rev. Lett. 88, 175501 (2002).

[121] R. H. Ewoldt, Master's thesis, Massachusetts Institute of Technology, 2006.

[122] C. O. Klein, H. W. Spiess, A. Calin, C. Balan, and M. Wilhelm, Macromolecules 40, 4250 (2007).

[123] K. S. Cho, K. Hyun, K. H. Ahn, and S. J. Lee, J. Rheol. 49, 747 (2005).

[124] R. Ewoldt, A. Hosoi, and G. McKinley, J. Rheol. 52, 1427 (2008).

[125] J. H. Conway, Groups, Combinatorics and Geometry, London Math. Soc. Lecture Notes Series [165], Proceedings of the L.M.S. Durham Symposium, July 515, Durham, UK, 1992.

[126] A. N. Beris, E. Stiakakis, and D. Vlassopoulos, A thermodynamically consistent model for the thixotropic behavior of concen-trated star polymer suspensions, 2007.

[127] S. Rogers, D. Vlassopoulos, and P. Callaghan, Phys. Rev. Lett. 100, 128304 (2008).

[128] D. Chopra, D. Vlassopoulos, and S. G. Hatzikiriakos, J. Rheol. 44, 27 (2000). 